\title{
Articular cartilage lesions : advances in conservative and surgical interventions
}

Citation for published version (APA):

Jansen, E. J. P. (2008). Articular cartilage lesions : advances in conservative and surgical interventions. [Doctoral Thesis, Maastricht University]. Maastricht University. https://doi.org/10.26481/dis.20081107ej

Document status and date:

Published: 01/01/2008

DOI:

10.26481/dis.20081107ej

Document Version:

Publisher's PDF, also known as Version of record

\section{Please check the document version of this publication:}

- A submitted manuscript is the version of the article upon submission and before peer-review. There can be important differences between the submitted version and the official published version of record.

People interested in the research are advised to contact the author for the final version of the publication, or visit the DOI to the publisher's website.

- The final author version and the galley proof are versions of the publication after peer review.

- The final published version features the final layout of the paper including the volume, issue and page numbers.

Link to publication

\footnotetext{
General rights rights.

- You may freely distribute the URL identifying the publication in the public portal. please follow below link for the End User Agreement:

www.umlib.nl/taverne-license

Take down policy

If you believe that this document breaches copyright please contact us at:

repository@maastrichtuniversity.nl

providing details and we will investigate your claim.
}

Copyright and moral rights for the publications made accessible in the public portal are retained by the authors and/or other copyright owners and it is a condition of accessing publications that users recognise and abide by the legal requirements associated with these

- Users may download and print one copy of any publication from the public portal for the purpose of private study or research.

- You may not further distribute the material or use it for any profit-making activity or commercial gain

If the publication is distributed under the terms of Article $25 \mathrm{fa}$ of the Dutch Copyright Act, indicated by the "Taverne" license above, 


\section{Articular cartilage lesions Advances in conservative and surgical interventions}




\section{Voor Nicole, Isa en Kas}

Layout and design:

D\&L graphics

www.dlgraphics.nl

\section{Coverdesign and Illustration:}

D\&L graphics

Printed by:

Drukkerij Bykorf

ISBN/EAN: 978-90-8590-031-3

\section{Copyright:}

(C) 2008 by E.J.P. Jansen, Maastricht, 2008

All rights reserved. No part of this publication may be reproduced or transmitted in any form or by any means, electronic or mechanical, including photocopy, recording, or any information storage and retrieval system, without permission in writing from the copyright owner. 


\title{
Articular cartilage lesions Advances in conservative and surgical interventions
}

\author{
Proefschrift
}

ter verkrijging van de graad van doctor aan de Universiteit Maastricht, op gezag van Rector Magnificus, Prof. mr. G.P.M.F. Mols

volgens het besluit van het College van Decanen,

in het openbaar te verdedigen

op vrijdag 7 november 2008 om 10.00 uur

door

\section{Edwin Juan Pieter Jansen}

Geboren op 13 januari 1973 te Boxmeer 


\section{Promotores:}

Prof. dr. G.H.I.M. Walenkamp

Prof. dr. S.K. Bulstra (Universitair Medisch Centrum Groningen)

\section{Copromotores:}

Dr. R. Kuijer (Universitair Medisch Centrum Groningen)

Dr. L.W. Van Rhijn

\section{Beoordelingscommissie:}

Prof. dr. H. Van Mameren (voorzitter)

Dr. J.P.M. Cleutjens

Prof. dr. W.J.A. Dhert (Universitair Medisch Centrum Utrecht)

Prof. dr. R.G.T. Geesink

Prof. dr. P.P. Geusens

The printing of this thesis was financially supported by:

Nederlandse Orthopaedische Vereniging, TiGenix, Biomet, Mölnlycke Health Care, MSD, Spronken Orthopedie, Smeets loopcomfort, Janssen-Cilag, Heraeus Medical, Bauerfiend Benelux, Stryker Nederland, Genzyme, Synthes, Frans Arts installaties Afferden, Defauwes-Habets Orthopedische Schoentechniek, Smith\&Nephew, Arthrex Nederland, B\&CO, Tramedico, Siemens Nederland, Stichting Kliniek en Wetenschap Orthopaedie Maastricht, SproFit, Orthopaedie 2000, het Anna Fonds, LIVIT Orthopedie, Tornier, DePuy, a Johnson\&Johnson company

The study described in this thesis was supported by grant BTS00021 from SenterNovem. 


\section{Contents}

$\begin{array}{lll}\text { Chapter } 1 & \text { General introduction } & 7\end{array}$

$\begin{array}{lll}\text { Chapter } 2 & \text { Aims of this thesis } & 27\end{array}$

Chapter 3 Development of partial-thickness articular cartilage $\quad 35$

injury in a rabbit model

Chapter 4 One intra-articular injection of hyaluronan prevents cell death and improves cell metabolism in a model of injured articular cartilage in the rabbit

Chapter 5 PEOT/PBT based scaffolds with low mechanical properties improve cartilage repair tissue formation in osteochondral defects

Chapter 6 Human periosteum-derived cells from elderly

patients as source for cartilage tissue engineering?

Chapter $7 \quad$ Assessing infection risk in implanted tissue-engineered devices

Chapter 8 Hydrophobicity as a design criterion for polymer scaffolds in bone tissue engineering

Chapter 9 General discussion

Chapter 10 Summary

Nederlandse samenvatting van de conclusie

References

Dankwoord

Curriculum vitae 


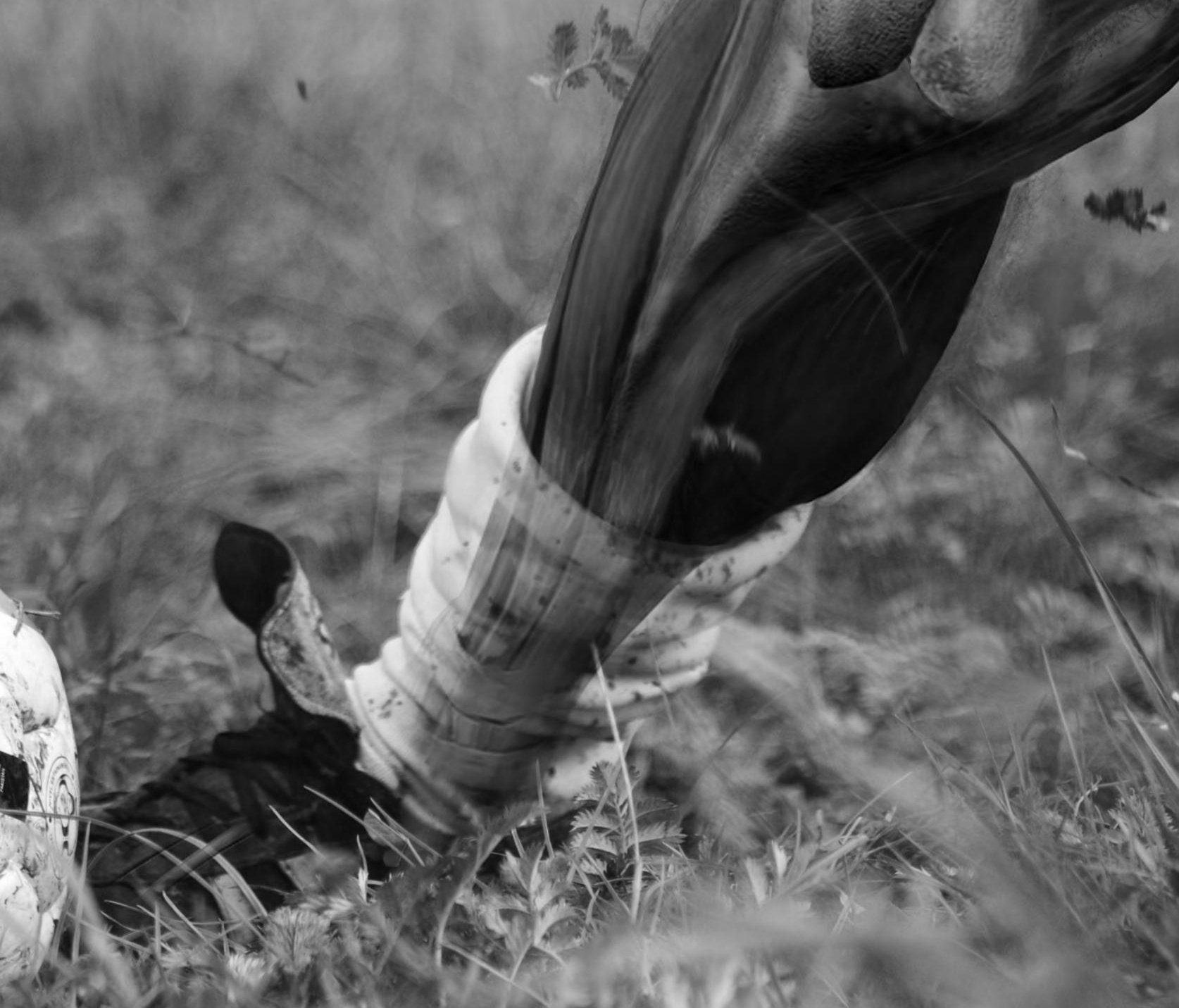




\section{CHAPTER 1}

\section{General Introduction}


Chapter 1 


\section{Articular cartilage lesions}

Articular cartilage lesions (Figure 1) can give rise to pain, joint effusion, locking phenomena and a limited range of motion. Onset is mainly connected with sports activity, and occurs commonly with concomitant articular lesions.

The reported incidences of cartilage lesions with knee arthroscopies are high. ${ }^{1-7}$ Hjelle et al. conducted a prospective study of cartilage lesions in 1,000 consecutive knee arthroscopies. ${ }^{7}$ Focal chondral or osteochondral defects were found in $19 \%$ of the arthroscopies. Of these defects $80 \%$ were single. Widuchowski et al. analyzed retrospectively more than 25,000 knee arthroscopies. ${ }^{6}$ Chondral lesions were found in $60 \%$. Isolated lesions, without concomitant articular lesion, accounted for $30 \%$. Most frequent localizations of cartilage lesions were the patellar articular surface and the medial femoral condyle.

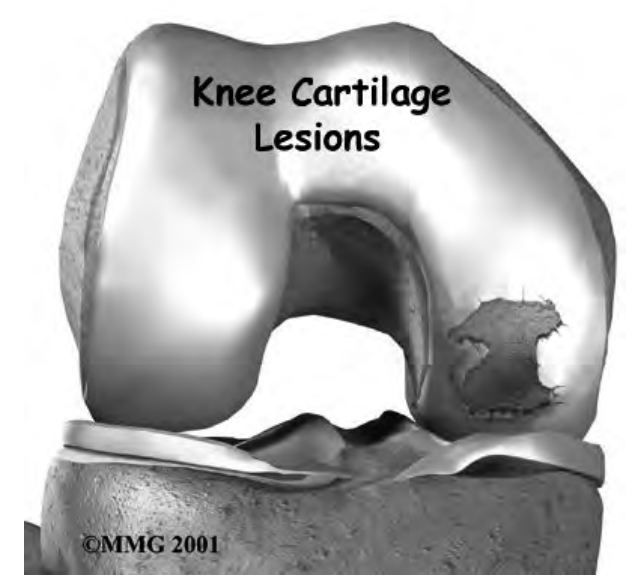

Figure 1. Articular cartilage defect on femoral condyle. Image courtesy of Medical Multimedia Group LLC, www.eOrthopod.com (for full-colour figure see page 193)

It is essential to discriminate between (i) chondral defects and (ii) osteochondral defects:

(i) Chondral defects are limited to the cartilage layer itself and occur either as partial- or full-thickness defects. Partial-thickness cartilage defects are entirely located within the cartilage, including having cartilage at the base of the defect. Full-thickness cartilage defects extend down to, but not into, the subchondral bone. ${ }^{8,9}$ Chondral defects are characterized by the absence of 
spontaneous repair. Since cartilage is avascular, the classic response of the body to damage is not available; the damaged tissue will not be removed by granulocytes and macrophages. Besides, since the chondrocytes are captured within the extracellular matrix, the proliferative capacity and the ability of cells to move towards the injury site are limited.

(ii) Osteochondral defects extend into the subchondral bone. ${ }^{8,9} \mathrm{~A}$ repair response is initiated as access to blood and mesenchymal stem cells from the bone marrow enter the defect. ${ }^{10}$ From rabbit studies it is clear that the osteochondral defect will be filled with fibrocartilage that does not have the same mechanical and chemical properties as the original articular cartilage. ${ }^{10-13}$

Cartilage injury results in chondrocyte necrosis and apoptosis close to the lesion. ${ }^{10,}{ }^{14-20}$ The earliest signs of apoptosis appear around 6 hours post-injury and the percentage of apoptotic cells increase up to 7 days after injury. ${ }^{24}$ Furthermore, the increased oxidative stress results in accelerated chondrocyte senescence. ${ }^{21-23}$ Altogether, the insufficient number of cells and inadequate cell activity cannot provide tissue repair and prevent degradation. Joint homeostasis alters, ${ }^{25-27}$ and the surrounding cartilage and underlying bone undergo progressive changes. Ultimately, it is generally accepted that premature osteoarthritis will develop in the affected joint. ${ }^{10,12,13,16,28-36}$

The rationales for repairing cartilage lesions are clinical pain relief, restoration of joint function, and prevention of degeneration in the affected joint. The management of cartilage defects can be distinguished in a conservative or surgical one.

The purpose of this chapter is to discuss the structure and function of articular cartilage, and the current available conservative and surgical treatment options when cartilage is damaged. 


\section{FUNCTION AND STRUCTURE OF ARTICULAR CARTILAGE}

Articular cartilage is a load bearing tissue that covers the subchondral bone in synovial joints.

It holds no vascular, lymphatic or neural tissue and is composed of a small number of chondrocytes embedded within a large amount of highly structured extracellular matrix (Figure 2). ${ }^{37}$ The major component of the extracellular matrix is water $(70-75 \%$ of the weight in adult tissue), while collagen and proteoglycans account for the major portion of the dry weight (17-19\% and 5$10 \%$ of the wet weight, respectively). ${ }^{38,39}$ The mechanical properties of articular cartilage are highly dependent on the integrity of the collagen network, the retention within the network of a high concentration of proteoglycans, and the capacity of chondrocytes to maintain this extracellular matrix integrity.

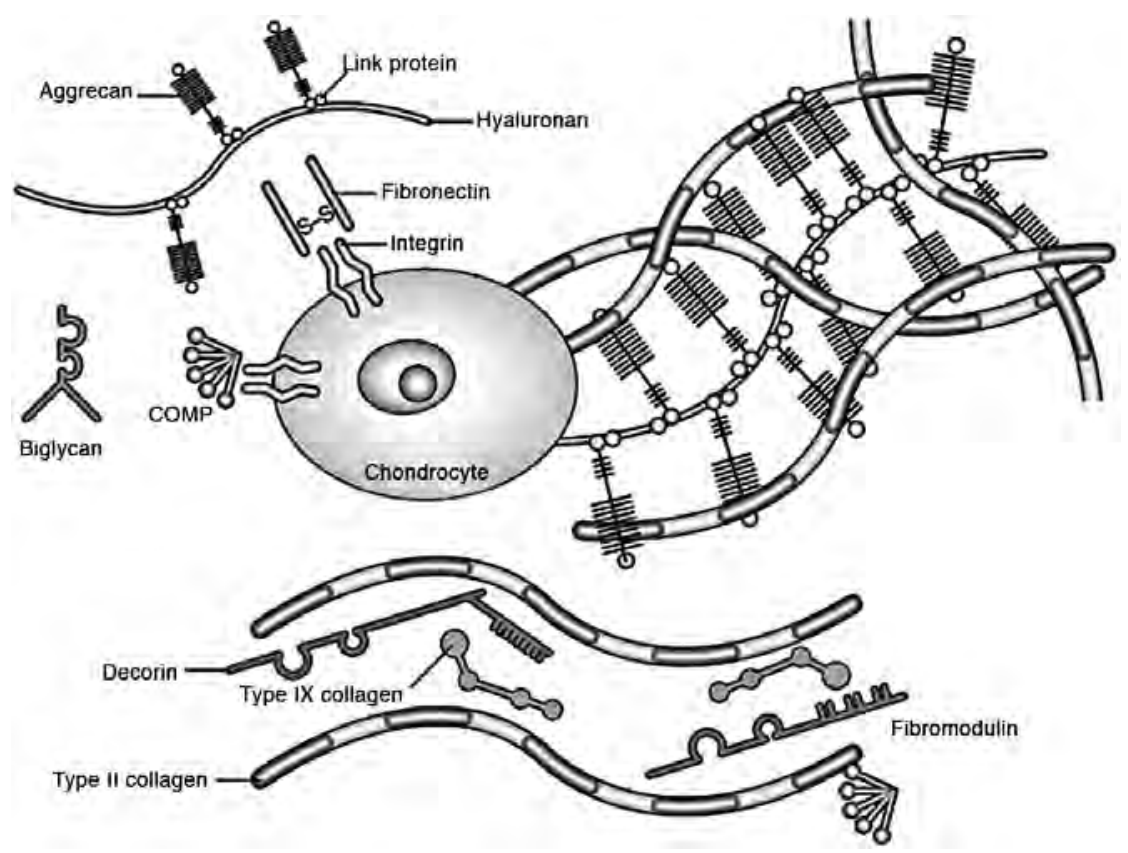

Figure 2. Extracellular matrix of cartilage. Adapted by permission from Macmillan Publishers Ltd: Chen FH et al. Technology Insight: adult stem cells in cartilage regeneration and tissue engineering. Nature Clinical Practice Rheumatology;2:373-382, copyright 2006.

(for full-colour figure see page 193) 


\section{Chondrocytes}

The overall cell volume density in human articular cartilage of the medial femoral condyle is approximately $1.7 \% .{ }^{40}$ Chondrocytes are responsible for the production and assembly of the constituents of the extracellular matrix of cartilage. Nutrition of the chondrocytes occurs by diffusion from the synovial fluid and the underlying subchondral bone. In younger animals, chondrocytes proliferate and divide rapidly. However, after skeletal maturity, chondrocytes do not divide anymore. ${ }^{41}$

\section{Collagens}

Approximately $95 \%$ of the collagen present in articular cartilage is collagen type $1{ }^{42}$ Other minor collagens present in articular cartilage are mainly collagen types VI, IX and $\mathrm{XI}^{43}$ The collagen network defines the form and tensile strength of articular cartilage.

\section{Proteoglycans}

The predominant proteoglycan in articular cartilage is aggrecan, comprising approximately $90 \%$ of the cartilage proteoglycans. It consists of a protein core to which the glycosaminoglycans are attached. Within cartilage four major types of glycosaminoglycans are distinguished: chondroitin sulphate, keratin sulphate, dermatan sulphate and hyaluronan. ${ }^{44,}{ }^{45}$ Each core protein contains approximately 50 keratin sulphate and 100 chondroitin sulphate chains. ${ }^{44}$

In addition to aggrecan, small proteoglycans as decorin, fibromodulin and biglycan are present in cartilage, some of which contain dermatan sulphate glycosaminoglycans. Note, glycosaminoglycans are not only situated in proteoglycans; also collagen type IX, which represents about $5-20 \%$ of the total collagen in cartilage, contains chondroitin or dermatan sulphate, and thus is collagen as well as proteoglycan. ${ }^{46}$

The proteoglycans are immobilized in the collagen network, which results in fixation of strong negative electric charges within the cartilage matrix. To balance this negative charge, cations are drawn into the tissue thus creating a large osmotic potential. Consequently, water is imbibed into the tissue thereby generating a large swelling force. In healthy articular cartilage approximately $20 \%$ of the total water-binding capacity of the system is used. ${ }^{47}$ In the unloaded condition, the swelling pressure is counteracted by constraining forces of the collagen network to prevent unlimited expansion. ${ }^{48}$ On compressive loading of the joint, water is squeezed out of the tissue. During unloading, cartilage rapidly recovers its elasticity as water is drawn back into the matrix by the hydrophilic 
proteoglycans, ${ }^{49}$ thus providing the unique resilience of articular cartilage. ${ }^{38,47}$ Several minor constituents that are neither collagens nor proteoglycans are present in articular cartilage. As an example, cartilage oligomeric matrix protein (COMP) associates with collagen and is believed to play a role in collagen fibrillogenesis. ${ }^{50}$ 


\section{Conservative treatment options}

Initial management of most patients is usually conservative. ${ }^{51}$ This may combine analgesics with nonpharmacological therapy such as physical therapy, bracing, orthoses, and ambulatory aids. ${ }^{52}$ The aim of physical therapy treatment is to improve pain control and improve physical capacity. This might be achieved by increased muscle strength, improved balance and coordination of movements, and better joint mobility. ${ }^{53,54}$ Changes in daily work and recreational activities may also be necessary. Obesity is a known risk factor for knee osteoarthritis and weight loss has been shown to slow the progression of the disease. ${ }^{55}$

Non-steroidal anti-inflammatory drugs and corticosteroid injections are considered by many physicians to be the preferred agents for the pharmacological management of osteoarthritis. Recent additions to the options for pharmacological therapy have included biological compounds, such as hyaluronans, chondroitin sulphate and glucosamine. ${ }^{56}$ The pharmacological treatment options will be discussed next.

\section{Non-steroidal anti-inflammatory drugs (NSAIDs)}

NSAIDs are among the most commonly used pharmacotherapeutic agents worldwide. NSAIDs inhibit cyclooxygenase (COX), which is an enzyme that converts arachidonic acid to prostaglandin $\mathrm{H}_{2}$. It can provide pain relief and reduce symptoms of inflammation. Randomized controlled trials attest to the superior efficacy of NSAIDs compared with placebo. ${ }^{57,58}$

However, there are certain disadvantages of routinely using NSAIDs in osteoarthritis. For example, all NSAIDs are associated with significant potential toxicity, particularly in the elderly population. ${ }^{59}$ The classical COX-inhibiters are non-selective and can cause peptic ulceration and dyspepsia due to direct irritation of the gastric mucosa, and inhibition of prostaglandin synthesis, which has a protective role in the gastrointestinal tract. COX-2 selective inhibitors have much less gastric irritation, leading to a decreased risk of peptic ulceration, but have been associated with an increased risk for cardiovascular disease. ${ }^{60}$ One of these agents, rofecoxib, was withdrawn from the market in 2004 due to these concerns. Since that time, numerous studies have illustrated that many of the NSAIDs, both the COX-2 selective inhibitors and the traditional NSAIDs, are associated with a moderately increased risk of cardiovascular events.

\section{Glucosamine and chondroitin sulphate}

Glucosamine and chondroitin sulphate are natural substances, and are building blocks of proteoglycans. 
In animal models glucosamine sulphate has been shown to normalize cartilage metabolism, rebuild experimentally damaged cartilage, and demonstrate mild anti-inflammatory properties. ${ }^{61,} 62$ There appears to be controversy as to the relative efficacy of glucosamine, and as to whether glucosamine can indeed modify the progression of osteoarthritis. ${ }^{63}$ In a Cochrane review effects of glucosamine, NSAID, or placebo in 20 randomized clinical trials over 2570 patients with osteoarthritis of the knee or hip were compared. ${ }^{64}$ Most of the studies were 2 to 3 months long. In the high quality studies, neither of the WOMAC outcomes of pain, function nor stiffness showed a superiority of glucosamine over placebo. Glucosamine produces similar symptomatic benefits as NSAIDs, but with a much lower probability of adverse reactions.

A multicenter, double-blind, placebo- and celecoxib-controlled glucosamine/chondroitin Arthritis Intervention Trial (GAIT) evaluated their efficacy and safety as a treatment for knee pain from osteoarthritis. ${ }^{65}$ The glucosamine and chondroitin sulphate alone or in combination failed to show any overall efficacy. However, exploratory analyses suggested that the combination of glucosamine and chondroitin sulphate may be effective in the subgroup of patients with moderate-to-severe knee pain.

\section{Intra-articular injection with corticosteroids}

Corticosteroid injection is recommended, particularly, when obvious signs of local inflammation with joint effusion are present. ${ }^{66,67}$ The response is generally rapid: relief of pain and inflammation starts one week after injection and can last for three to four weeks. ${ }^{68}$ Longer term benefits have not been confirmed.

\section{Intra-articular injection with hyaluronan}

Viscosupplementation is an intra-articular therapeutic modality based on the physiologic importance of hyaluronan in synovial joints. Hyaluronan products, while slower in onset of action, may offer a more durable response with improvement in pain, range of motion and patient global assessment than corticosteroids provide. While corticosteroids and hyaluronan show similar beneficial effects at one to four weeks post injection, hyaluronan is more effective than corticosteroids between five and thirteen weeks post injection. ${ }^{66}$, ${ }^{67}$ Its efficacy is comparable to systemic forms of active intervention, with more local reactions but fewer systemic adverse events. ${ }^{69-72}$ It is unclear however how hyaluronan exerts its positive effect. 


\section{SURGICAL TREATMENT OPTIONS}

\section{Arthroscopic lavage and debridement}

During arthroscopy fluid is flushed through the knee, which cleans the knee of debris and inflammatory enzymes (lavage). Besides, unstable chondral flaps, torn meniscal fragments, hypertrophied synovium, and loose bodies are removed by using mechanical instruments (debridement). The subchondral bone is left intact. ${ }^{73,74}$ Numerous uncontrolled, retrospective case series have reported substantial pain relief. However, a randomized, placebo-controlled trial did not show any improvement in relieving pain or improving function outcomes during 24 months after the procedure. ${ }^{75}$

\section{Subchondral plate penetration}

Penetration of the subchondral layer is a low-cost and minimally invasive procedure. The subchondral bone layer is breached to create access of blood and mesenchymal stem cells from the bone marrow to the lesion. All elements necessary for a classical wound healing response are then introduced for chondrogenic repair.

Various techniques of perforating the subchondral bone layer have been suggested:

\section{- $\quad$ Drilling}

Pridie was the first to describe drilling techniques in which the defect is drilled in pinpoint fashion. ${ }^{76}$ The repair cartilage which subsequently fills the drill holes has been shown to include both hyaline and fibrocartilage. The best indications for this technique are acute small to medium partial-thickness lesions on the weight-bearing portion of the femoral condyles. ${ }^{77}$

\section{- Abrasion arthroplasty}

Arthroscopic abrasion arthroplasty is an elaborate description for an extensive multiple tissue debridement, including synovectomy, chondroplasty, meniscectomy and osteophyte removal. The abrasion portion of the surgical procedure is done only in the area of exposed bone by removing the entire superficial layer of subchondral bone plate (Figure 3). Areas of intact degenerative articular cartilage are not abrased. Patients are allowed nonweight-bearing ambulation until 2 months postoperatively. ${ }^{78,79}$

This procedure is indicated in older patients with severe diffuse degenerative arthritis, loss of joint space, and exposed sclerotic lesions who seek an alternative to total knee replacement. In a high percentage of patients a definitive operation can be postponed. Most studies reported a hyaline-like cartilage, but deterioration over time with changes to fibrocartilage occurred. ${ }^{80}$ 


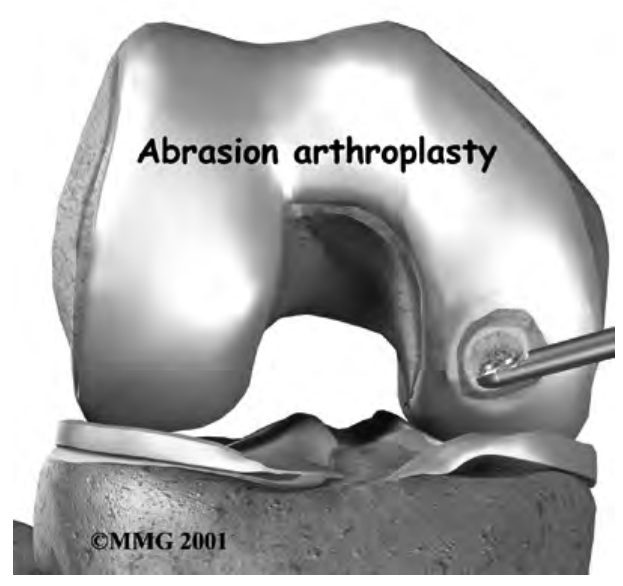

Figure 3. Abrasion arthroplasty. Image courtesy of Medical Multimedia Group LLC, www.eOrthopod.com (for full-colour figure see page 194)

\section{- Microfracture}

Microfracture is a single stage arthroscopic procedure, which is currently the most frequently applied technique to perforate the subchondral layer.

First the base of the defect is debrided of soft tissue, after which the calcified cartilage layer is removed from the subchondral bone with shaver or curette. Unstable cartilage remnants are debrided. Then arthroscopic awls are used to make multiple holes, 3 to $4 \mathrm{~mm}$ apart, penetrating 3 to $4 \mathrm{~mm}$ into the subchondral bone of the affected region (Figure 4). Subsequently the tourniquet is released to confirm visualization of blood and/or fat droplets out of the holes. The specific rehabilitation program varies based on location of the chondral defect. $^{81}$ The continuous passive motion machine (CPM) is used for 6 to 8 hours per day for 8 weeks together with patellar mobilizations, passive flexion/ extension exercises and quadriceps sets and straight leg raises. Patients are allowed touch-down weight bearing until 8 weeks postoperatively.

Significant improvements in function and symptoms from time of microfracture to the final follow-up of 4 years were noted. ${ }^{82-84}$ At seven years after the treatment, $80 \%$ of the patients have significant reduction of symptoms. ${ }^{85}$ Clinical outcomes of microfracture were worse in lesions larger than $2 \mathrm{~cm}^{2}{ }^{86}$ The location of the defect is also an important issue: microfracture has less favourable results when it was used to treat patellofemoral lesions. ${ }^{87}$ 


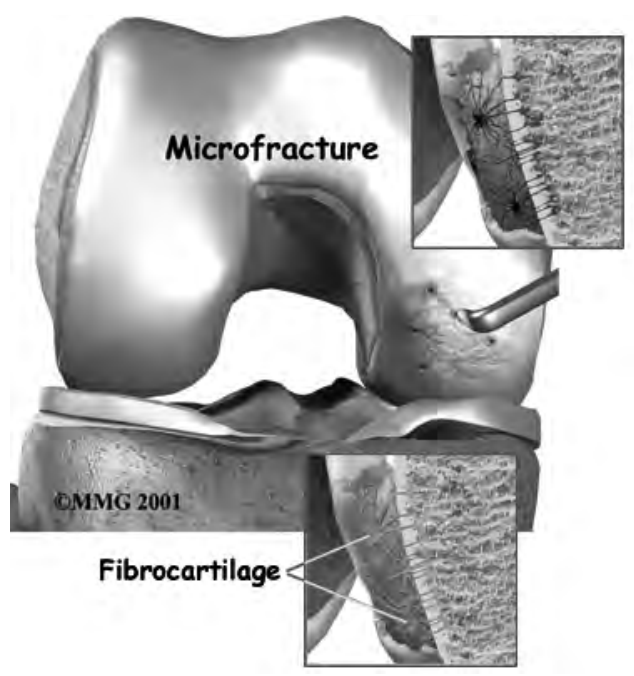

Figure 4. Microfracture. Image courtesy of Medical Multimedia Group LLC, www.eOrthopod.com (for full-colour figure see page 194)

- Spongialization

Since 1975, spongialization was performed by Ficat. ${ }^{88}$ This procedure resects, en bloc, all of the diseased cartilage with its corresponding subchondral bone, leaving a completely exposed cancellous bony bed. This procedure was thought to be superior to drilling alone since the subchondral plate itself is abnormal and often of poor quality. In addition a depression was created in which the new tissue could regenerate without the adverse effect of weightbearing load imposed by the opposite cartilage surface. Another reason for removing the subchondral plate was the elimination of the source of pain, since the subchondral bone is well innervated and sensitive to pressure transmitted by softened cartilage.

Postoperative management includes isometric quadriceps exercises and mobilization of the knee joint in a pool. Weight-bearing may be started by the fifth to seventh postoperative day.

\section{Correction osteotomies}

Varus or valgus malalignment are significant predisposing factors for focal degenerative lesions of the articular surface and predict decline in physical function. ${ }^{89}$ With a correction osteotomy (Figure 5), first reported by Jackson, ${ }^{90}$ the limb is realigned to unload the injured cartilage surfaces. Postoperative weight-bearing is allowed in plaster after 4 to 6 weeks. Movement of the knee 

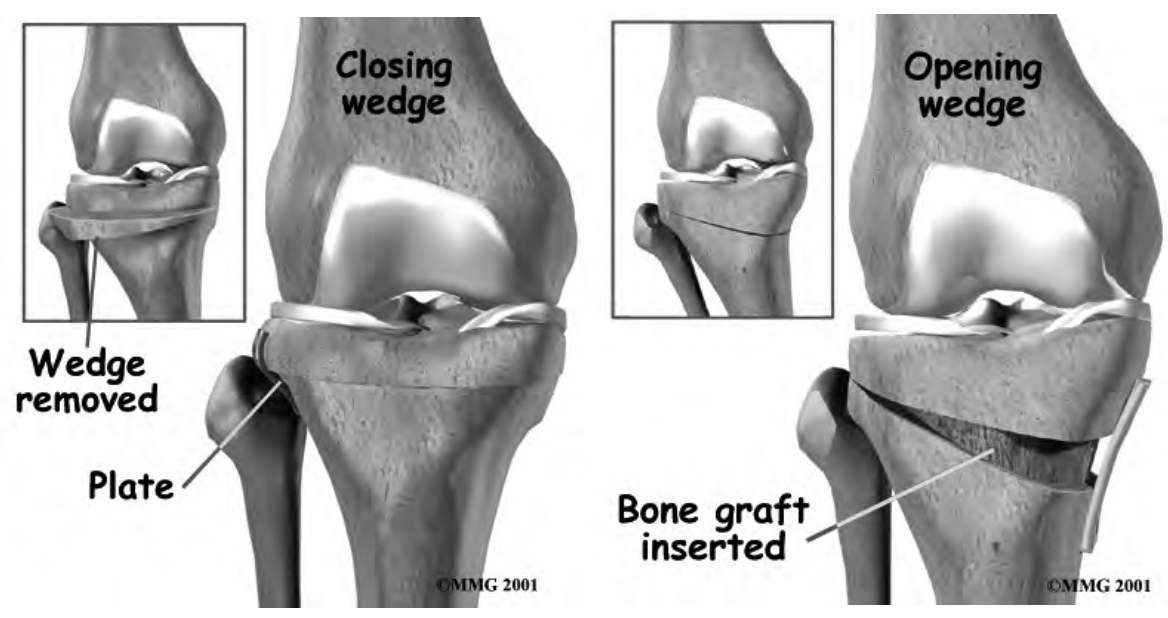

Figure 5. Correction osteotomies. Image courtesy of Medical Multimedia Group LLC, www.eOrthopod.com (for full-colour figure see page 195)

can be started after 8 to 10 weeks. ${ }^{90}$ This technique is reserved for those patients with unicompartmental cartilage damage thought to be young for joint replacement. ${ }^{91}$ Favourable candidates for osteotomy are younger than 60 years of age, and have less than $12^{\circ}$ of angular deformity, pure unicompartmental disease, absence of a lateral tibial thrust, ligamentous stability, and a preoperative range of motion arc of at least $90^{\circ}{ }^{92,}, 93$ It has to be taken into account that in an osteoarthritic knee the metabolism of articular cartilage as a whole is affected, ${ }^{94}$ and thus a pure unicompartimental disease does not exist. Symptoms decrease, ${ }^{95-98}$ but only temporary and partial, with results lasting for approximately 5 years. ${ }^{91,} 99,100$ There is no evidence whether an osteotomy is more effective than conservative treatment. ${ }^{101}$ Osteotomy with subsequent subchondral drilling yields better results compared to osteotomy alone, but after 2 to 9 years the differences disappear. ${ }^{102}$

\section{Joint distraction}

Joint distraction is based on the hypothesis that osteoarthritic cartilage has some reparative activity when the damaged cartilage is mechanically unloaded. By using an external fixation frame further wear and tear of the articular cartilage is prevented and chondrocytes are allowed to initiate repair. Furthermore, distraction relieves mechanical loading of the peri-articular bone, which results in osteopenia. After joint distraction, less dense subchondral bone will absorb greater stress, resulting in lower stress on the overlaying cartilage. The intermittent synovial fluid 
pressure is maintained by using hinges, thin flexible wires, or springs in the distraction frame. ${ }^{103}$ Clinical studies on joint distraction are limited, but in turn are promising in treatment of severe ankle osteoarthritis with long-term benefit. ${ }^{104-107}$

\section{Osteochondral grafts}

Articular cartilage is restored by transplanting osteochondral allografts or autologous grafts to the debrided lesion.

\section{- Allografts}

An important advantage of this technique is that the graft can be taken from approximately the same site as where the recipient's defect is. This provides for a graft with exactly the same thickness, contour and compliance. A disadvantage is the concomitant risks of transmitting infectious diseases. To eliminate the risk to a major extent, freeze drying of the graft can be performed. This reduces the immunogenicity, but also decreases the viability of the transplanted chondrocytes. ${ }^{108,109}$

\section{- Autologous grafts}

Multiple osteochondral arthroscopic transplantation was first reported by Matsusue. ${ }^{110}$ One large osteochondral autologous graft or multiple smaller cylinders (mosaicplasty) are harvested from minimal weight-bearing areas of the distal femur, for example the lateral edge of the lateral femoral condyle, and transplanted to the debrided cartilage lesion (Figure 6).

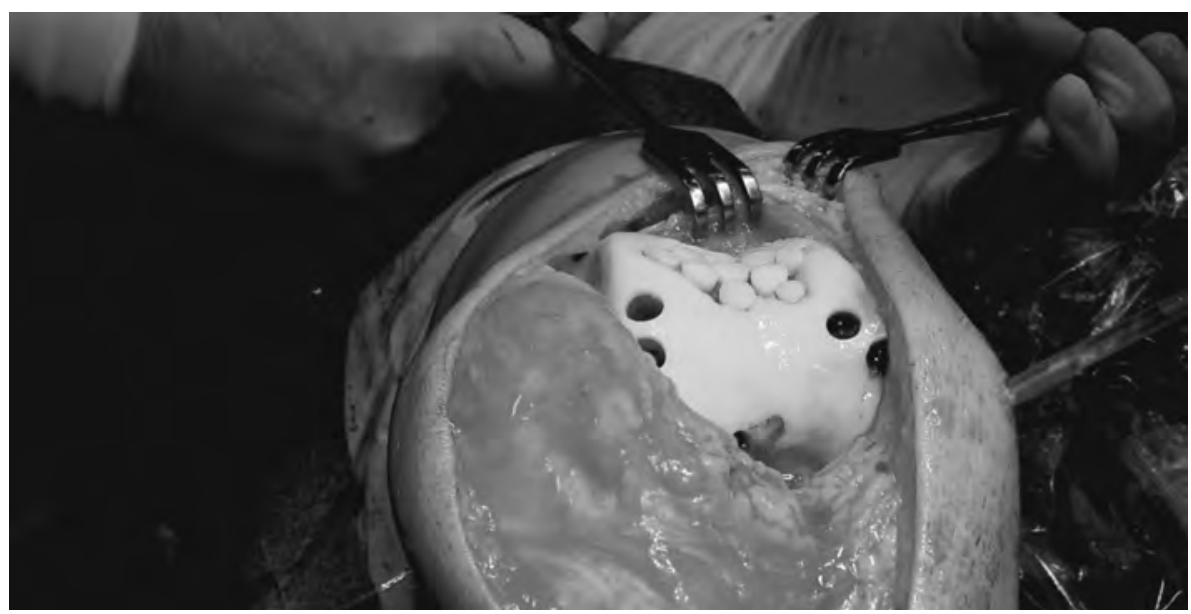

Figure 6. Mosaicplasty. Reprinted with permission from Hangody L and Fules P. Autologous Osteochondral Mosaicplasty for the Treatment of Full-Thickness Defects of Weight-Bearing Joints: Ten Years of Experimental and Clinical Experience. J. Bone Joint Surg. Am., 2003: 8525-32. (for full-colour figure see page 195) 
Rehabilitation after autologous osteochondral mosaicplasty permits an immediate full range of motion, but requires 2 weeks of non-weight-bearing and an additional 2 to 3 weeks of partial weight-bearing after the operation. ${ }^{111}$

Good-to-excellent clinical outcomes were achieved in $91 \%$ of the patients at 3 to 6 years of follow-up. ${ }^{110,112}$ Donor sites were filled with cancellous bone and fibrocartilage as seen during second-look arthroscopies. Disadvantages are the limited supply of tissue, the adverse effect on joint function, and the difficulty of matching the topology of the donor graft to the defect site. Although the bone plugs integrate very well with the subchondral bone, there is no integration at the cartilage level. ${ }^{113},{ }^{114}$ Donor-site morbidity remains a major concern, especially in patients with larger defects. For best results with mosaicplasty, an upper patient age limit of 50 years, ${ }^{112}$ and defects that are between $1-4 \mathrm{~cm}^{2},{ }^{111}$ are recommended.

\section{Autologous chondrocyte implantation (ACI)}

This technique was first proposed by Grande, ${ }^{115}$ and in 1987 the first patient was treated. ${ }^{2,116}$ A cartilage biopsy is harvested arthroscopically from a less-load bearing area of the injured knee, minced and enzymatically digested. Isolated cells are culture expanded in vitro for several weeks to obtain sufficient cells. During a second procedure an arthrotomy is performed. The subchondral plate is cleaned, but not penetrated, and damaged cartilage surrounding the defect is excised. The cultured chondrocytes $\left(2 \times 10^{6}\right.$ cells per $\mathrm{cm}^{2}$ defect area) are injected in the cartilage lesion underneath a periosteal membrane that is sutured on the defect in order to captivate the chondrocyte suspension. Fixation of the periosteal tissue is secured by a watertight seal with fibrin glue.

The original method relied on a sutured periosteal cover with the cambium layer facing into the defect. Today resorbable membranes are often used instead of periosteum.

CPM and a gradually increased weight-bearing status are the initial steps of the rehabilitation process. Isometric quadriceps training, straight leg raises and hamstring strengthening should be introduced early and progressively advanced to resisted exercises. Progressive closed chain exercises start from 3 weeks postoperatively. Open chain exercises can be initiated around the eighth week. ${ }^{117}$

It is recommended that the defect area is between 1 and $10 \mathrm{~cm}^{2}$ and that patients are between 15 and 55 years of age. ${ }^{118}$ Good clinical results were reported even at long-term follow-up. ${ }^{2,119-121}$ The major complications are periosteal hypertrophy, detachment of the periosteal flap, arthrofibrosis and transplant failure. ${ }^{122}$ Besides, suturing of articular cartilage, as is done with the 
periosteal flap to captivate the injected chondrocytes, induces severe local damage, which is progressive and reminiscent of that associated with the early stages of osteoarthritis. ${ }^{123}$

A modification on the $\mathrm{ACl}$ technique is characterized chondrocyte implantation $(\mathrm{CCl})$ in which a cell population capable of making stable hyalinelike cartilage is used. As in $\mathrm{ACl}$ the characterized cells are injected underneath an autologous periosteal flap.

\section{Periosteal arthroplasty}

Whole periosteal grafts are harvested and transplanted to the osteochondral defect with the cambium layer, containing the chondrocyte precursor cells, facing up into the joint. Clinical experience has been limited, with encouraging results. ${ }^{124-126}$ This technique is still being defined in terms of its indications and results, and is primarily studied at one center. ${ }^{127}$ Donor factors that are important include the harvest site, the size of the periosteal explant, and the age of the donor. The highest chondrogenic potential was seen from periosteum from the iliac crest. Within the tibia, the upper and middle zones of the proximal region were similar and were slightly better than the lower proximal tibia or the distal tibia. ${ }^{128}$

\section{Perichondrial arthroplasty}

Autologous perichondrium including its chondrogenic layer from the cartilaginous part of a lower rib is harvested. The graft is fixated with fibrin glue on the debrided cartilage lesion with the chondral side facing the joint. ${ }^{129}$ Shortcomings are calcification and delamination of the graft at long-term follow-up. Furthermore, perichondrial arthroplasty did not show better clinical results than open debridement and drilling after 10 years. ${ }^{130}$ 


\section{Cartilage tissue engineering}

The term "tissue engineering" itself was introduced in 1987, and defined as "techniques that apply the principles of biology and engineering to the development of functional substitutes for damaged tissue" ${ }^{131}$ Cartilage tissue engineering aims at repairing cartilage defects with constructs that both functionally and biologically resemble the surrounding tissue.

Note the above described $\mathrm{ACl}$ is the first tissue engineering application introduced in the clinic. A variation of the original periosteum-cover technique $(\mathrm{ACl}-\mathrm{P})$ includes the use of a cover manufactured from porcine-derived type I/type III collagen (ACl-C). In both techniques the implantation of cultured chondrocytes in suspension is used. The next generation of tissue engineering applications is matrix-induced autologous chondrocyte implantation (MACI). The MACI membrane consists of a porcine type I/type III collagen bilayer seeded with chondrocytes. ${ }^{132}$ In a randomised trial treatment of isolated symptomatic osteochondral defects in the knee with $\mathrm{ACl}-\mathrm{C}$ or $\mathrm{MACl}$ were compared. ${ }^{133}$ Both treatments resulted in significant improvements to the clinical score within one year. The frequency of good to excellent functional outcomes was higher for $\mathrm{MACl}$ than for ACl-C. However, improvements to the modified Cincinnati knee score, the VAS and the Stanmore functional score were not significantly different between $\mathrm{ACl}-\mathrm{C}$ and $\mathrm{MACl}$. There was no significant difference between the arthroscopic appearance of the graft and the histological findings.

\section{Artificial scaffolds}

Tissue engineering application with scaffolds makes use of three-dimensional porous biomaterials. The ideal scaffold is biocompatible; has a porous network into which surrounding tissue can be induced; acts as a temporary template for the growth of new tissue meanwhile facilitating the proper tissue organization; exhibits the appropriate surface chemistry for cell attachment; withstands physiological loading such that the strength of the scaffold is retained until the regeneration tissue can assume its structural role; and is permeable to permit the ingress of nutrients and elution of waste products. ${ }^{134,135}$

\section{Cells}

Scaffolds without cells; or scaffolds in which cells are seeded can be used. A possible source of cells is the chondrocytes residing in the cartilage itself. Small amounts of articular cartilage can be harvested from less load-bearing areas of the affected knee. The limited amount of harvested donor cells is expanded in vitro using monolayer cultures, which is a process in which chondrocytes dedifferentiate. When sufficient cells are obtained (approximately 15 to 20 
million cells ${ }^{132}$ ), the dedifferentiated chondrocytes have to redifferentiate to produce the proper extracellular matrix with adequate mechanical properties. ${ }^{136-}$ ${ }^{139}$ It is a prerequisite that chondrocytes maintain redifferentiation capacity during expansion, which in turn is a major limiting factor in successful cartilage tissue engineering. Then the expanded cells are seeded into the scaffold and cultured in vitro. Subsequently, the tissue-engineered constructs can be implanted in the defect.

An alternative cell type, instead of the chondrocyte, can be the mesenchymal stem cell, which can be found in various tissues including bone marrow, trabecular bone, dermis, periosteum, perichondrium, umbilical cord, umbilical cord blood, adipose tissue, synovium, skeletal muscle, liver, placenta and peripheral blood. ${ }^{140-145}$ Even the superficial zone of articular cartilage contains a pluripotent progenitor cell type. ${ }^{146}$ These cells could be used as an extra-articular cell source for cartilage tissue engineering applications so that no morbidity in the knee is associated. However the numbers of stem cells present in a host decline rapidly with older age. ${ }^{147-150}$ 
General Introduction 


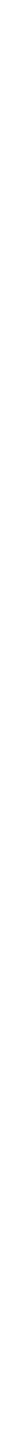




\section{CHAPTER 2}

Aims of this thesis 
Chapter 2 
An ideal treatment for articular cartilage lesions would result in regeneration of new hyaline cartilage in the area of the defect which is integrated with surrounding normal cartilage and mechanically functional.

Despite extensive experimental and clinical data on the repair of damaged cartilage, none of the conservative or surgical treatment options has led to a repair tissue that closely resembles native cartilage. Usually the repair tissue comprises of fibrocartilage, which is known to be biochemically and biomechanically different from hyaline cartilage. Moreover, often the repair tissue deteriorates over time. ${ }^{151}$ Short-term results, concerning pain relief and mobility, are nearly always promising, but in turn last only temporarily. At best they can eliminate or at least delay the need for an artificial joint prosthesis.

Thereof, data from in vitro and in vivo research are needed to further optimize available and novel articular cartilage repair techniques.

\section{Natural course of articular cartilage defect in rabbits}

Numerous cartilage repair procedures have been and are being developed. Such procedures often are tested in animal models usually in symptom-free joints, whereas isolated lesions in patients can be present for a considerable time before treatment occurs. The discrepancies between successfully tested cartilage repair techniques in animals and the less favourable outcomes in patients could be explained by the chronic disturbances in human joint homeostasis related to the delay in treatment. ${ }^{33,152,153}$

Therefore, an animal model that better reflects the clinical situation, including an extended period of preoperative cartilage damage, ${ }^{25}$ would be better suited for evaluating experimental cartilage repair techniques. However, the natural course of cartilage surrounding an isolated cartilage lesion in the often-used acute rabbit model is largely unknown and may not resemble the clinical setting with chronic alterations. We questioned whether these lesions led to deterioration of surrounding cartilage macroscopically and microscopically, and disturbances in proteoglycan metabolism reflecting degenerating articular cartilage. (Chapter 3)

\section{Conservative treatment options - Hyaluronan}

Hyaluronan has received a great deal of attention as a potential agent of intervention in osteoarthritis. However, clinical studies on the effect of hyaluronan are controversial.

In previous animal experiments we have shown that one injection with 
high molecular weight hyaluronan restored the metabolism of chondrocytes that was inhibited by the irrigation solution. ${ }^{154,155}$ While these experiments were done in anatomically normal cartilage, in a clinical setting cartilage metabolism is often negatively influenced by cartilage lesions and a disturbed joint homeostasis. ${ }^{25}$

We questioned whether hyaluronan restores chondrocyte metabolism in knee joints with longer lasting lesions, better reflecting the clinical situation, when arthroscopy is first performed weeks to months after the original injury. Besides, we questioned whether hyaluronan could exert a chondro-protective effect for the chondrocytes next to a fresh defect, such as the ones created during arthroscopic shaving procedures. (Chapter 4)

\section{Surgical treatment options - Cartilage tissue engineering using scaffolds}

Unfortunately, the current therapeutic strategies do not predictably restore a durable articular surface, and have not yet been proven to be efficacious in preventing osteoarthritis. Therefore, research has been focused on repair of articular surfaces by tissue engineering applications using scaffolds.

The synthetic materials by far most applied in manufacturing of copolymer scaffolds for cartilage tissue engineering studies are poly $(\alpha)$-hydroxyesters such as poly(glycolic acid), poly(lactic acid) and poly(lactic-co-glycolic acid). We focused primarily on the application of a block copolymer comprised of poly(ethylene oxide terephthalate) (PEOT) and poly(butylene terephthalate) (PBT). A major advantage of these biocompatible and biodegradable copolymers is that by varying the amount and the length of the two building blocks a whole range of polymers can be obtained with differences in surface properties, swelling capacity, degradability and mechanical strength. These materials have an extensive safety record and have received FDA and CE approvals.

PEOT/PBT copolymers were extensively tested in vitro. ${ }^{156-159}$ The PEOT/PBT 70/30 composition showed promising results in vitro concerning chondrocyte attachment, proliferation and differentiation, ${ }^{160}$ whereas the PEOT/PBT 55/45 composition most closely matched the biomechanical properties of native articular cartilage. ${ }^{161,162}$

The in vivo healing response of osteochondral defects using PEOT/PBT based porous scaffolds is unknown. We questioned whether PEOT/PBT scaffolds would improve the performance of tissue engineered cartilage. (Chapter 5)

With the objective of using scaffolds in cartilage tissue engineering applications in the human setting, also scaffolds in vitro seeded with cells can 
be used. As mentioned in Chapter 1 mesenchymal stem cells could be used for this purpose.

The heterogeneous nature of bone marrow ${ }^{163,164}$ and adipose tissue $\mathrm{e}^{165}$ confounds the results of various therapies and often necessitates isolation and purification of the mesenchymal stem cells. In contrast, periosteum is a relatively pure source of chondrogenic or osteogenic precursor cells ${ }^{166-168}$ as its histological structure is relatively simple. Besides, it can be obtained with minimal morbidity. It contains two distinct layers: a thick outer fibrous layer, adherent to a thin inner cambium layer in which the mesenchymal stem cells reside.

However, with increasing age the thickness and chondrogenic capacity of the cambium layer diminishes, ${ }^{169,} 170$ which would make periosteum a less promising cell source for use in the repair of cartilage defects using scaffolds. Thereof we investigated whether periosteum-derived cells from elderly patients could be expanded and redifferentiated into a chondrogenic lineage. (Chapter 6)

Biomaterial-associated infections in general are low incidence, but because of their extensive significance and increasing complications across all device categories, such infections represent a substantial total clinical case load annually, high cost burdens on the health care system for mitigation, and enormous patient discomfort and not infrequently, death.

While the risk in traditionally implanted biomaterials is well-recognised, the occurrence of infection in polymer scaffold tissue engineering is virtually unknown. In the last years we implanted over 200 PEOT/PBT based scaffolds in rabbit knee osteochondral defects. Hence, we questioned whether the infection incidence of the polymer scaffolds is as high as that from traditionally implanted biomaterials. (Chapter 7)

Despite extensive research in the field of scaffold tissue engineering, our fundamental understanding of the role of the scaffold biomaterial is still rather limited. The variety of concepts and models so far investigated by different groups for the generation of osteochondral grafts reflects that understanding of the requirements to restore a normal joint function is still poor. While in principle it would be feasible to develop technical solutions to a well-defined design, the principles of the design itself still have to be defined.

A possible way to expand our understanding of scaffold materials may be to dissect the various factors that determine its ultimate success. Such information can easily be obscured when degradable scaffolds are used, in which metabolites cause and maintain a chronic inflammatory process and even may be cytotoxic. 
One approach is to study scaffolds which do not decompose. Non-degrading porous biomaterials provide an important tool to expand our comprehension of the role of biomaterials in scaffold-based tissue engineering approaches. Evaluation of the performance of such scaffolds may shed new light on the importance of the choice of the material. We investigated whether hydrophobicity of the biomaterial is an important design criterion for polymeric scaffolds. (Chapter 8) 


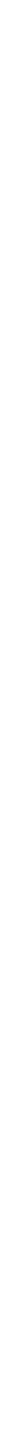




\section{CHAPTER 3}

\section{Development of partial-thickness articular cartilage injury in a rabbit model}

E.J.P. Jansen, P.J. Emans, L.W. Van Rhijn, S.K. Bulstra, R. Kuijer

Clinical Orthopaedics and Related Research. 2008 Feb;466(2):487-494 


\section{Abstract}

In humans, partial-thickness cartilage lesions frequently result in premature osteoarthritis. While rabbits often are used as a model for partial-thickness cartilage lesions, the natural course of cartilage surrounding such a lesion is largely unknown. We developed a rabbit model of a chronic partial-thickness cartilage defect and asked whether these defects led to (1) deterioration of surrounding cartilage macroscopically and microscopically (increased Mankin score) and (2) disturbances in proteoglycan metabolism.

In 55 rabbits, we created a 4-mm-diameter partial-thickness cartilage defect on one medial femoral condyle. The surrounding cartilage was characterized during the course of 26 weeks. Contralateral knees were sham-operated.

In experimental knees, we found cartilage softening and fibrillation at 13 and 26 weeks. High Mankin scores observed at 1 week were partially restored at 13 weeks but worsened later and were most pronounced at 26 weeks. Mankin scores in the experimental groups were worse at 1 and 26 weeks when compared with the sham groups. Mankin scores at 26 weeks improved compared with 1 week in the sham groups. Disturbances in proteoglycan metabolism were less evident.

In this rabbit model, a partial-thickness cartilage lesion resulted in early markers of degenerative changes resembling the human situation. 


\section{INTRODUCTION}

Clinically, preventing joint degeneration is an important rationale for repairing cartilage defects. Early diagnosis and treatment of patients are recommended to prevent progression to advanced osteoarthritis (OA). However, most chondral defects are not symptomatic ${ }^{4,171}$ and therefore exist for some time before they are treated. Furthermore, the period of preoperative symptoms before repair of the chondral defect often is extended. In our clinic, the mean duration of symptoms before a defect was treated was 29 months (range, 4-48 months) and 23 months (range, 3-48 months) for perichondrium transplantation and open débridement and drilling, respectively. ${ }^{153}$ In one study, the mean duration of symptoms for treating osteochondritis dissecans by autologous chondrocyte transplantation was 7.8 years (range, $0.1-36$ years). ${ }^{120}$ The extended duration between the occurrence of a cartilage defect and its treatment in humans will likely negatively influence the outcome of cartilage repair owing to changes in joint homeostasis. ${ }^{25}$

In contrast to the human setting, in some animal studies, the cartilage lesions were treated immediately after creation of the defect. ${ }^{172,}{ }^{173}$ The discrepancies between successfully tested cartilage repair techniques in animals and the less favorable outcomes in patients could be explained by the chronic disturbances in human joint homeostasis relating to the delay in treatment. ${ }^{33,152,153}$ Therefore, an animal model that better reflects the clinical situation, including an extended period of preoperative cartilage damage, ${ }^{25}$ would be better suited for evaluating experimental cartilage repair techniques.

Full-thickness cartilage defects smaller than $3 \mathrm{~mm}$ in diameter in a rabbit model reportedly regenerate spontaneously. ${ }^{174}$ However, few studies have focused on alterations in cartilage surrounding a partial-thickness articular cartilage defect. Lu et al. ${ }^{175}$ reported ongoing degeneration of cartilage surrounding the defect in a sheep model during the course of 52 weeks. Because rabbits often are used as a model to test novel cartilage repair techniques, Hunziker and Quinn ${ }^{176}$ reported a considerable number of chondrocytes were lost from cartilage adjacent to surgically created partial-thickness articular cartilage defects, whereas the synthetic activity of the remaining chondrocytes remained unchanged. However, those authors created cartilage lesions with a width of $1 \mathrm{~mm}$, which we believe is too small for testing current cartilage repair techniques.

We describe a rabbit model in which chronic partial-thickness articular cartilage defects were created with a diameter of $4 \mathrm{~mm}$. We asked whether these lesions led to (1) deterioration of surrounding cartilage macroscopically 
(loss of glossy appearance) and microscopically (increased Mankin scores) and (2) disturbances in proteoglycan (PG) metabolism (increase in PG synthesis rate and inability of the cartilage matrix to retain newly synthesized PGs) reflecting degenerating articular cartilage.

\section{MAterials AND METHODS}

We followed macroscopic, histologic, and biochemical changes during the course of 26 weeks to reflect degenerative changes in articular cartilage surrounding 4-mm defects created on the medial femoral condyles of 553 month-old New Zealand white rabbits (females; average weight, $2.5 \mathrm{~kg}$; range, $1.5-3.0 \mathrm{~kg}$ ). Contralateral knees were sham-operated.

From previous experiments and the literature, ${ }^{25,} 29$ the sample size was determined based on a difference of 1.5 points in the Mankin score (as described by Mankin et al. ${ }^{19}$ ) using the power analysis of Sachs, ${ }^{177}$ with a power of $80 \%$, two-tailed, and a confidence interval of $95 \%$. This resulted in a minimum of five knees per group for histologic evaluation. For analysis of early changes in PG synthesis rate and ability of the cartilage matrix to retain newly synthesized PGs, 12 knees per group for each follow-up were chosen. ${ }^{29}$ Two rabbits were excluded from analysis; one rabbit died of pneumonia and the second had a knee infection. One sample was lost (Table 1).

Table 1. Rabbit Demographics

\begin{tabular}{ccccccc} 
Follow-up (weeks) & Number of Rabbits & Weight $(\mathrm{kg})^{*}$ & \multicolumn{3}{c}{ Number of Knees } \\
& & & \multicolumn{2}{c}{ Histology } & Biochemistry \\
& & & Sham & Defect & Sham & Defect \\
\hline 1 & 18 & $2.6(0.12)$ & 6 & 5 & 12 & 12 \\
13 & 17 & $3.7(0.46)$ & 5 & 5 & 12 & 12 \\
26 & 18 & $4.4(0.44)$ & 6 & 6 & 12 & 12 \\
\hline
\end{tabular}

*Values are expressed as means, with standard deviations in parentheses

The experiments were conducted following the national and European guidelines for animal experiments. The Maastricht University Committee for Animal Experiments approved all experimental protocols.

Preoperatively, each rabbit was fasted for 12 hours. General anaesthesia was induced by intramuscular injection of $35 \mathrm{mg}$ ketamine hydrochloride per $\mathrm{kg}$ body weight and $5 \mathrm{mg}$ xylazine per $\mathrm{kg}$ and maintained throughout the surgical 
procedure by administration of $2 \%$ halothane and a mixture of oxygen and nitrous oxide delivered by an automatic ventilator using a specially designed mask. Preoperatively, all rabbits received an intramuscular injection of $10 \mathrm{mg}$ ceftiofur per $\mathrm{kg}$.

Arthrotomy of the tibia-femoral articulation was performed through a medial longitudinal parapatellar incision. The patella was dislocated laterally to expose the surface of the medial femoral condyle. A 4-mm-diameter skin biopsy punch (KAI Europe $\mathrm{GmbH}$, Solingen, Germany) was used to circumscribe the defect centered on the weight-bearing part of the medial femoral condyle. Noncalcified cartilage was removed from the outlined defect using a scalpel (defect group) to create a partial-thickness defect. Special care was taken to prevent penetration of the subchondral bone. The contralateral knees (sham group) received an arthrotomy followed by lateral dislocation of the patella, as performed in the experimental knees but without creation of a defect. At the end of the procedure in the defect and the sham groups, the patellae were relocated and the wound was closed in layers.

Postoperative pain relief was provided by administering $50 \mu \mathrm{g}$ buphenomorphine per $\mathrm{kg}$ at 2 hours and 1 day. The rabbits were housed in a cage for 2 days, after which they were allowed to have unlimited activity in groups in a stable. They were fed a standard rabbit diet and had water ad libitum. They were euthanized 1, 13, and 26 weeks after surgery with an overdose of pentobarbital.

For macroscopic purposes, the femoral condyles were dissected and photographed. The lesions were evaluated for whether they were healed, and cartilage of the medial femoral condyles was examined for cartilage softness and fibrillation (indicated by a loss of glossy appearance). Then condyles were prepared either for histologic grading or for determination of PG synthesis and PG retention capacity of the cartilage surrounding the defects using $\left[{ }^{35} \mathrm{~S}\right]$ sulphate incorporation ex vivo.

For histologic analysis, condyles were fixed in a $10 \%$ formalin solution for 5 days at $4^{\circ} \mathrm{C}$. After decalcification in a $10 \%$ EDTA solution, samples were dehydrated in a series of increasing concentrations of ethanol and embedded in 2-hydroxyethyl methacrylate (Technovit 7100; Heraeus Kulzer $\mathrm{GmbH}$, Wehrheim, Germany). Sections of $5 \mu \mathrm{m}$ were cut along the midsagittal plane using a multirange microtome (LKB, Stockholm, Sweden) and stained with thionine. All sections were viewed at the same time by two individuals (EJJ, RK) who were blinded to group assignment. The articular cartilage on the entire width of the medial femoral condyle was evaluated using the histologic and histochemical grading system of Mankin et al. ${ }^{178}$ Lower scores indicate better histologic appearance. 
For biochemical analysis, the cartilage was harvested from the medial femoral condyles under aseptic conditions and transferred to preweighed tubes containing $1 \mathrm{~mL}$ medium (Dulbecco's Modified Eagle's Medium/Ham's F12 nutrient mix with GlutaMAX ${ }^{\mathrm{TM}}$ I; Invitrogen, Breda, The Netherlands) supplemented with ascorbic acid 2-phosphate $(0.2 \mathrm{mmol} / \mathrm{L}$; Sigma-Aldrich Chemie BV, Zwijndrecht, The Netherlands), penicillin (100 U/mL), streptomycin $(100 \mu \mathrm{g} / \mathrm{mL})$, and amphotericin $(0.25 \mu \mathrm{g} / \mathrm{mL})$ (Invitrogen). In the defect group, the cartilage 2 to $3 \mathrm{~mm}$ proximal and distal of the created defect was dissected. The medium was discarded and $500 \mu \mathrm{L}$ medium supplemented with $3.7 \times 10^{5}$ $\mathrm{Bq} \mathrm{Na}_{2}{ }^{35} \mathrm{SO}_{4}$ (Amersham Biosciences Benelux, Roosendaal, The Netherlands) $\left({ }^{35} \mathrm{~S}\right]$ sulphate medium) per $\mathrm{mL}$ was added. The samples were incubated in a humidified $\mathrm{CO}_{2}$ incubator overnight. The $\left[{ }^{35} \mathrm{~S}\right]$ sulphate medium was removed and cartilage samples were washed for 10 minutes three times with $1 \mathrm{~mL}$ sterile phosphate-buffered saline.

One half of the samples were used for analysis of the PG synthesis ${ }^{179}$ and the other half for analysis of the PG retention capacity.

To determine PG retention, cartilage samples were cultured under normal conditions in the presence of $10 \%$ fetal bovine serum for an additional 48 hours. ${ }^{180}$ The cartilage samples were completely digested in a solution containing $0.15 \mu \mathrm{g}$ proteinase $\mathrm{K}$ (Merck-Europe BV, Amsterdam, The Netherlands) per $\mu \mathrm{L}, 0.1 \mu \mathrm{g}$ PG (A1 fraction isolated from human articular cartilage) per $\mu \mathrm{L}, 50 \mathrm{mmol}$ Tris- $\mathrm{HCl}\left(\mathrm{pH}\right.$ 7.9) per $\mathrm{L}$, and 1 mmol $\mathrm{CaCl}_{2}$ (MerckEurope BV) per $\mathrm{L}$ in a shaking water bath at $56^{\circ} \mathrm{C}$ for 3 days. After centrifugation at 3,000 $\mathrm{g}$ for 5 minutes, the DNA content of the supernatants was assessed using a commercially available assay kit (CyQUANT ${ }^{\circledR}$ DNA assay kit; Invitrogen) according to the manufacturer's instructions. In brief, $200 \mu \mathrm{L}$ CyQUANT ${ }^{\circledR}$ GR dye/cell lysis buffer was added to each sample. An aliquot of each sample was incubated for 5 minutes at room temperature protected from light exposure. The sample fluorescence was measured at 480-nm excitation and 520-nm emission wavelengths. Fluorescence measurements were compared with the values obtained from a standard DNA curve, and the resulting DNA content was normalized to the cartilage wet weight.

The remaining supernatant was supplemented with cetylpyridinium chloride (Merck-Europe BV) and $\mathrm{NaCl}$ to a final concentration of $0.5 \%(\mathrm{w} / \mathrm{v})$ and $0.2 \mathrm{~mol} / \mathrm{L}$, respectively. Samples were incubated at $37^{\circ} \mathrm{C}$ for 1 hour to precipitate the glycosaminoglycans, which then were centrifuged at 15,000 $g$ for 5 minutes. The supernatants were discarded and the pellets were washed once with $100 \mu \mathrm{L}$ of a solution of $0.1 \%$ cetylpyridinium chloride in $0.2 \mathrm{~mol} \mathrm{NaCl}$ per $\mathrm{L}$ and then dried. Pellets were dissolved in $100 \mu \mathrm{L}$ formic acid (Merck-Europe BV) at room 
temperature for 24 hours. A $10-\mu \mathrm{L}$ aliquot of each sample was mixed with 2.5 $\mathrm{mL}$ Formula 989 scintillation fluid (DuPont, Dordrecht, The Netherlands) and counted in a liquid scintillation counter. The total $\left[{ }^{35} \mathrm{~S}\right]$ sulphate incorporation of each cartilage sample was calculated using the specific activity of the medium and was normalized to the cartilage wet weight.

Data were not normally distributed and therefore were analyzed using nonparametric tests. First, data were analyzed using the Kruskal-Wallis (nonparametric one-way analysis of variance) and Friedman overall tests. Then, a two-tailed Mann-Whitney $U$ test was performed to compare differences in Mankin score, including all its individual parameters (structure, cells, matrix, tidemark) and PG metabolism (PG synthesis and PG retention capacity) between treated (having a previously created cartilage lesion) and sham-treated knees. A $p$ value less than 0.05 was considered significant. All data were analyzed with SPSS Version 12.0.1 (SPSS Inc, Chicago, IL).

\section{Results}

The creation of partial-thickness articular cartilage lesions resulted in changes suggesting early degeneration: cartilage softening and fibrillation, indicated by loss of glossy appearance of the articular surface at 13 and 26 weeks. These changes were confirmed by histologic analysis (increased Mankin scores) and biochemical alterations (changes in PG metabolism). At 1 week (Figure 1A), the cartilage surrounding the defect had a glossy, white, smooth appearance, which disappeared at 13 (Figure 1B) and 26 weeks (Figure 1C). In addition, at 13 and 26 weeks, the articular surface around the created lesion showed signs of fibrillation. In the sham groups, no noticeable macroscopic abnormalities were
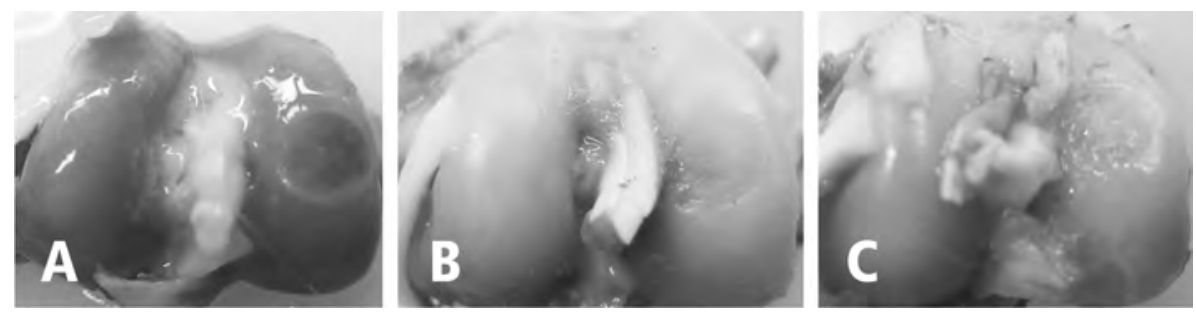

Figure 1. Representative photographs are shown of articular surfaces at (A) 1 week, (B) 13 weeks, and (C) 26 weeks after creating partial-thickness articular cartilage defects on rabbit medial femoral condyles. Cartilage surrounding the defect had a glossy, white, smooth appearance at 1 week, which disappeared during the course of 26 weeks. (for full-colour figure see page 196) 


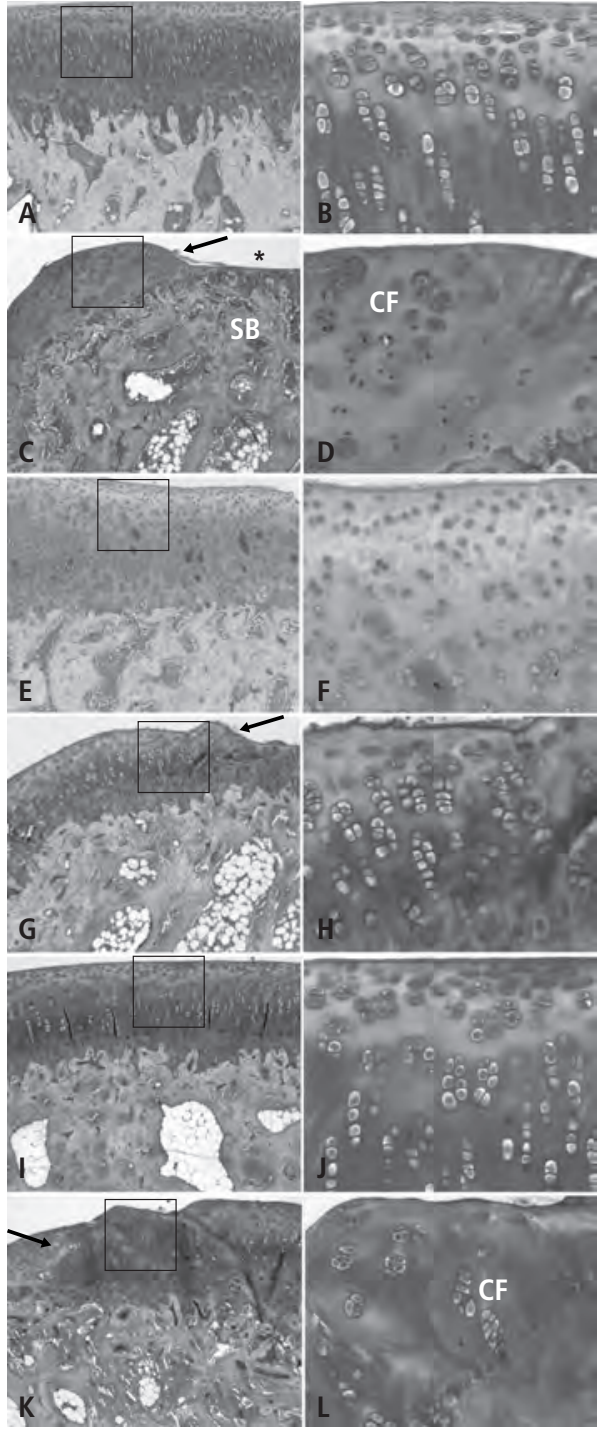

Figure 2. Photomicrographs of sections are shown at (A-D) 1 week, $(E-H) 13$ weeks, and (I-L) 26 weeks. (A) A sham-treated rabbit medial femoral condyle at 1 week follow-up (Stain, thionine; original magnification, $\times 100$ ); (B) an enlargement of the box in (A) (Stain, thionine; original magnification, $\times 400$ ); and (C) a condyle with partialthickness articular cartilage defect at 1 week follow-up are shown (Stain, thionine; original magnification, $\times 100)$. The cartilage $\operatorname{defect}(*)$ did not penetrate the subchondral bone (SB); (D) An enlargement of the box in (C) is shown (Stain, thionine; original magnification, $\times 400$ ). A cluster formation (CF) can be seen. (E) A sham-treated rabbit medial femoral condyle at 13 weeks follow-up (Stain, thionine; original magnification, $\times 100)$; (F) an enlargement of the box in (E) (Stain, thionine; original magnification, $\times 400)$; (G) a condyle with a partial-thickness articular cartilage defect at 13 weeks follow-up (Stain, thionine; original magnification, $\times 100)$; and $(\mathrm{H})$ an enlargement of the box in (G) are shown (Stain, thionine; original magnification, $\times 400$ ). (I) A sham-treated rabbit medial femoral condyle at 26 weeks follow-up (Stain, thionine; original magnification, $\times 100$ ); (J) an enlargement of the box in (I) (Stain, thionine; original magnification, $\times 400)$; and $(\mathrm{K})$ a condyle with partial-thickness articular cartilage defect at 26 weeks follow-up are shown (Stain, thionine; original magnification, $\times 100$ ). The partial-thickness articular cartilage defect was not healed at 26 weeks. Cartilage surrounding the defect showed surface irregularities; (L) an enlargement of the box in $(\mathrm{K})$ is shown (Stain, thionine; original magnification, $\times 400)$. A cluster formation (CF) can be seen. The arrows in (C), (G), and (K) indicate the edge of the defect. (for full-colour figure see page 197) 
observed during the course of 26 weeks (not shown), but histologic analysis revealed minor degenerative changes (Figure 2). We found higher Mankin scores in the defect groups at 1 week $(p=0.030)$ and 26 weeks $(p=0.024)$ compared with the sham groups (Figure 3 ).

At 1 week, the sham group (Figure $2 \mathrm{~A}-\mathrm{B})$ scored better $(p=0.036)$ than the defect group (Figure 2C-D) on the structure parameter (1.0 versus 2.0 for the sham and defect groups, respectively) (Table 2). Surface irregularities were

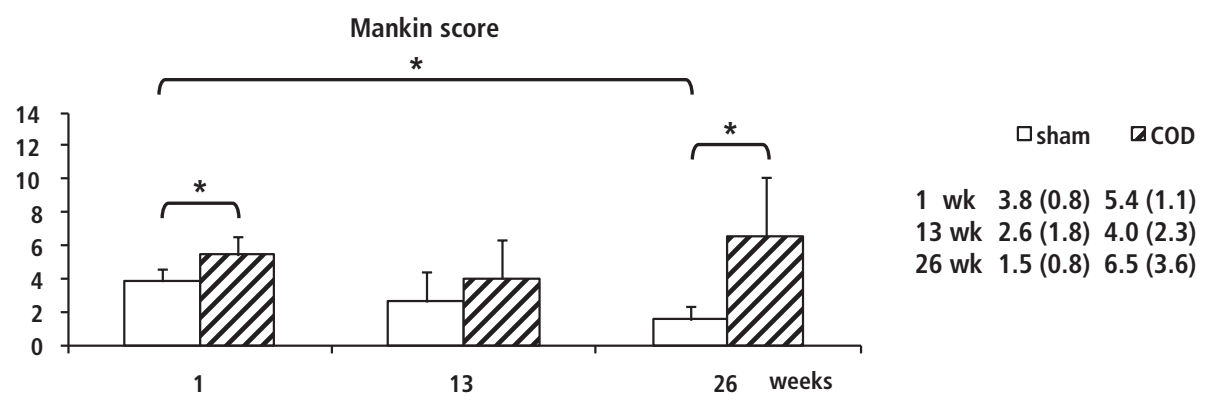

Figure 3. A histogram shows the degree of degenerative changes (using the Mankin score) in rabbit medial femoral condyles 1, 13, or 26 weeks postoperatively.

Worse histologic appearances were observed in the defect (COD) groups at 1 and 26 weeks when compared with the sham groups. The key shows means and standard deviations. ${ }^{*}=$ significant at $p<0.05$.

more pronounced in the defect group compared with the sham group. Cartilage cellularity was similar between sham and defect knees. In both groups, the matrix staining was reduced compared with the lateral femoral condyle and the tidemark integrity was disturbed.

Table 2. Mankin Scores at the Different Follow-up Times

\begin{tabular}{ccccccc} 
Mankin Parameter & \multicolumn{2}{c}{1 Week } & \multicolumn{2}{c}{13 Weeks } & \multicolumn{2}{c}{26 Weeks } \\
& Sham & Defect & Sham & Defect & Sham & Defect \\
\hline Structure & $1.0(0.0)$ & $2.0(1.2)$ & $1.2(0.4)$ & $1.8(1.8)$ & $1.0(0.0)$ & $2.8(1.8)$ \\
Cells & $0.0(0.0)$ & $0.8(1.3)$ & $0.0(0.0)$ & $0.0(0.0)$ & $0.0(0.0)$ & $1.7(1.4)$ \\
Matrix & $2.0(0.6)$ & $1.6(0.9)$ & $0.8(1.1)$ & $1.2(0.8)$ & $0.3(0.5)$ & $1.2(1.0)$ \\
Tidemark & $0.8(0.4)$ & $1.0(0.0)$ & $0.6(0.5)$ & $1.0(0.0)$ & $0.2(0.4)$ & $0.8(0.4)$ \\
\hline
\end{tabular}

Values are expressed as means, with standard deviations in parentheses 
At 13 weeks, we observed no differences between the groups. The articular surface remained irregular, whereas cartilage cellularity was normal in both groups. Reduced matrix staining and tidemark abnormalities also were observed at 13 weeks (Figure 2E-H).

At 26 weeks, the sham group scored better than the defect group in the structure $(p=0.022)$, cells $(p=0.022)$, and tidemark parameters $(p=0.027)$ (Table 2). In the defect group (Figure $2 \mathrm{~K}-\mathrm{L}$ ), the surface showed more clefts compared with the sham group (Figure 2I-J). We observed cell clusters embedded in slightly stained matrix, whereas the tidemark integrity remained disturbed. In the sham series, the histologic appearance of the cartilage at 26 weeks improved $(p=0.005)$ compared with 1 week (Figure 3$)$. The matrix ( $p=$ $0.004)$ and tidemark parameters $(p=0.027)$ also were improved (Table 2$)$.

The mean cartilage wet weights and DNA contents of defect- and shamtreated knees were similar (Table 3).

Between 13 and 26 weeks, we found a decrease $(p=0.045)$ in the PG retention capacity in defect-treated knees. However, we observed no differences in cartilage metabolism (PG synthesis and PG retention capacity) between cartilage from defect- and sham-treated knees at 1, 13, and 26 weeks (Table 3).

Table 3. Data on Metabolic Properties of Articular Cartilage at the Different Follow-up Times

\begin{tabular}{|c|c|c|c|c|c|c|c|c|}
\hline \multirow{2}{*}{$\begin{array}{l}\text { Follow-up } \\
\text { (weeks) }\end{array}$} & \multicolumn{2}{|c|}{ Wet Weight $(\mu \mathrm{g})$} & \multicolumn{2}{|c|}{ 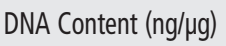 } & \multicolumn{2}{|c|}{ PG Synthesis (dpm/ $/ \mathrm{g}) \quad \mathrm{P}$} & \multicolumn{2}{|c|}{ PG Retention Capacity (dpm/ $/ \mu \mathrm{g})$} \\
\hline & Sham & Defect & Sham & Defect & Sham & Defect & Sham & Defect \\
\hline 1 & $6.7(3.6)$ & $7.3(5.0)$ & $2.1(1.9)$ & $2.1(1.4)$ & 2646 (1961) & 2615 (1959) & $814(504)$ & $270(110)$ \\
\hline 13 & $5.6(3.2)$ & $7.0(2.9)$ & $3.2(2.7)$ & $1.6(0.5)$ & $1915(1560)$ & $1237(383)$ & $423(306)$ & $295(122)^{*}$ \\
\hline 26 & $8.4(2.6)$ & $8.8(4.8)$ & $0.8(0.3)$ & $1.4(1.1)$ & $1039(271)$ & $1103(590)$ & 339 (223) & $175(90)^{*}$ \\
\hline
\end{tabular}

\section{Discussion}

In humans, untreated articular cartilage lesions often progress toward premature OA. This rationale for recent trends for early repair is to prevent the OA. Numerous novel repair procedures have been and are being developed for this purpose. Such procedures often are tested in animal models usually in symptomfree joints, whereas isolated lesions in patients can be present for a considerable time before treatment occurs. The natural course of cartilage surrounding an 
isolated cartilage lesion in the often-used acute rabbit model is largely unknown and may not resemble the clinical setting with chronic alterations. Therefore, we evaluated the effect of the lesion with time on the surrounding cartilage with regard to macroscopic (softening and fibrillation), microscopic (increased Mankin score), and biochemical (increased PG synthesis and inability to retain newly synthesized PGs) parameters.

This study has two major limitations. First, while economically and practically attractive, the rabbit model is not an entirely suitable animal model to study articular cartilage repair procedures in preclinical studies. ${ }^{181}$ Hunziker $^{181}$ noted "...the matrix domain sustained and remodelled by an individual cellular unit is, in the human, approximately 8 to 10 times larger than that in the rabbit." This likely would lead to substantial enhancement in the rabbit to maintain surrounding cartilage compared with the human. Nevertheless, the rabbit is probably the most often used model for economic reasons and the literature contains interpretations based on rabbit data. Although we believe our delayed rabbit model better represents the clinical situation, cartilage repair procedures using this model should still be interpreted with caution before proceeding to clinical studies. The second limitation is the power of the study, which was sufficient for histologic grading using the Mankin score but not sufficient for the biochemical parameters studied. Thus, we can describe only trends for the PG synthesis and the capacity of the cartilage matrix to retain newly synthesized PGs.

In this model, partial-thickness articular cartilage lesions with a 4-mm diameter did not heal during the course of 26 weeks and we found no signs of regeneration; these lesions therefore represent critical-size defects. We observed degenerative features macroscopically and microscopically at 13 and 26 weeks around the cartilage defects. Histologically, the degeneration observed 1 week postoperatively was partially reversed at 13 weeks but then tended to increase again from 13 to 26 weeks. We noted no progressive cartilage degeneration (as reflected by the Mankin scores) from 13 to 26 weeks. This probably is attributable to the slow progression of the degenerative process, as has been observed in other quadrupeds. In the dog, severe degeneration is first evident 5 years after initiation of the process. ${ }^{182}$ The time at which severe degeneration occurs in rabbits is unknown.

The sham-treated knee showed articular cartilage changes during the first weeks after the arthrotomy, which can be explained by the effects of the operation (eg, effect of exposure of room air, stress of the sutures in the 
relatively small joint $\left.{ }^{183-186}\right)$. During the course of 26 weeks, the cartilage appeared to fully recover as observed histologically. This suggests regeneration of the mild changes in sham-operated knees, which may be related to peculiarities of the rabbit model.

Cell density was diminished at the wound edge of the cartilage defects as observed histologically. However, we observed normal cellularity using biochemical measurements. These findings were consistent with results described by Hunziker and Quinn, ${ }^{176}$ who reported with quantitative autoradiographic analysis chondrocytes within $100 \mu \mathrm{m}$ of a partial-thickness defect had synthetic activity similar to that of cells far from the lesion. Furthermore, the DNA assay, although one of the best available yet, may not be sensitive enough to detect the cell death occurring in the edges of the cartilage defects. Therefore, normal cellularity, or even increased cell numbers, in remote areas might compensate for hypocellularity in the wound edges.

Biochemically, cartilage from experimental knees did not differ from cartilage in sham-treated knees, which could indicate cartilage surrounding a partial-thickness articular defect was biochemically normal. However, this is in contrast to our histologic findings suggesting degenerative changes during the course of 26 weeks after creating the defect. We noted a nonsignificant trend toward persistent loss in PG retention after creation of the defect and this might have contributed to the observed histologic degeneration. A possible explanation for this lack of difference is that in sham-treated knees persistent biochemical alterations take place in the first weeks because of the arthrotomy, as was observed histologically, and after 13 weeks because of alterations in joint homeostasis, occurring before histologic changes in cartilage degeneration.

Our model involves creation of one circumscribed partial-thickness articular cartilage lesion without concomitant injuries of the meniscus or anterior cruciate ligament. It has advantages compared with other animal models: (1) when similar diameters are used, the effect of cartilage repair techniques can be monitored without the confounding effects of other potential causes of cartilage degeneration; (2) the operation is relatively simple and creates circumscribed cartilage lesions; (3) repair of these chronic partial-thickness articular cartilage lesions occurs with surrounding degeneration, which resembles the clinical situation $^{4,12,187,174,10,188}$; and (4) cartilage lesions are created on the medial femoral condyle, which is the most commonly affected zone of articular cartilage damage observed with arthroscopies in humans. ${ }^{3,7,189}$ 
Existing animal models intended to replicate human OA fail to resemble the clinical situation of a focal cartilage lesion, whereas the permanent trigger for degeneration will interfere with attempts of cartilage repair or regeneration. ${ }^{190,} 191$ Damaging articular cartilage, as described in the groove model, ${ }^{29,} 192$ did not reflect a one-time trauma in the clinical setting. Furthermore, it would be challenging to reproduce exactly the same grooves in each animal as far as depth and length. Penetration of the subchondral bone, as described in the articular step-off model, ${ }^{187}$ allows migration of mesenchymal stem cells influencing the repair process. In addition, although these are models for an advanced stage of $\mathrm{OA}$, they are not expected to reflect the altered matrix metabolism and articular cartilage degeneration surrounding a focal partial-thickness articular cartilage lesion with time.

We report the evolution of cartilage changes surrounding a partialthickness articular cartilage defect in a rabbit model during the course of 26 weeks. We believe a delayed treatment model is important when exploring cartilage repair strategies to prevent degenerative changes. Our data suggest a defect at least 13 weeks old most likely resembles the clinical focal cartilage lesion that has failed to heal after an initial remodelling process. 


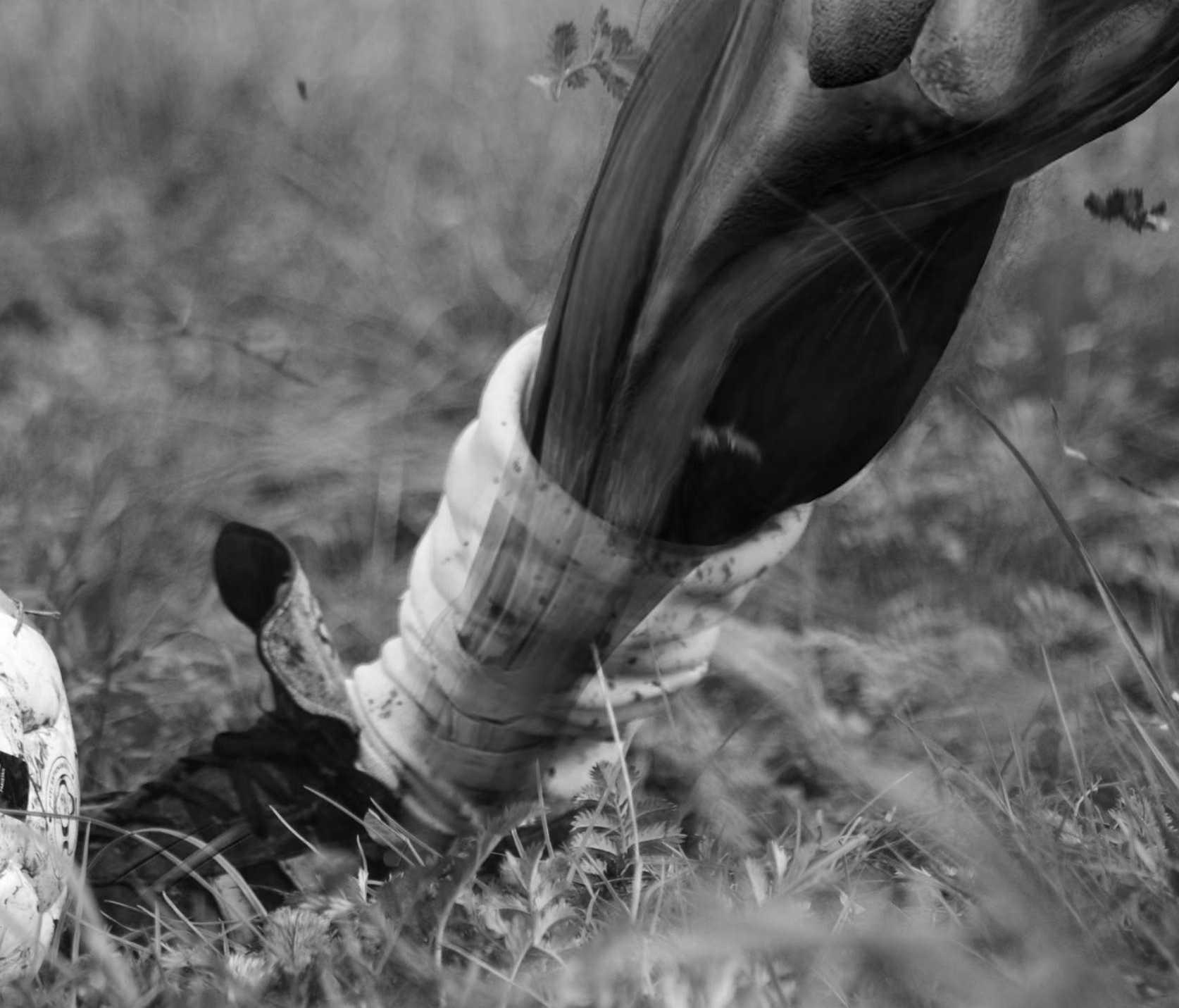




\section{CHAPTER 4}

\section{One intra-articular injection of}

hyaluronan prevents cell death and improves cell metabolism in a model of injured articular cartilage in the rabbit

E.J.P. Jansen, P.J. Emans, C.M. Douw, N.A. Guldemond, L.W. Van Rhijn, S.K. Bulstra, R. Kuijer

Journal of Orthopaedic Research. 2008 May;26(5):624-630 


\section{Abstract}

The purpose of this study was to determine the effect of one intra-articular injection of hyaluronan on chondrocyte death and metabolism in injured cartilage.

Twenty-three 6-month-old rabbits received partial-thickness articular cartilage defects created on each medial femoral condyle. In order to examine the effect on articular cartilage surrounding iatrogenic cartilage lesions, which can occur during arthroscopic procedures, study 1 was performed: In 14 rabbits both knees were immediately rinsed with $0.9 \% \mathrm{NaCl}$. Experimental knees were treated with hyaluronan. Six rabbits were sacrificed at 2 days; 8 rabbits 3 months postoperatively. Histomorphometric analysis was used for studying cell death in cartilage next to the defect.

In order to examine the effect on longer lasting lesions, more reflecting the clinical situation, study 2 was performed: After 6 months knee joints of 9 rabbits were i) irrigated with $0.9 \% \mathrm{NaCl}$, ii) treated with hyaluronan after irrigation with $0.9 \% \mathrm{NaCl}$, or iii) sham-treated. After 7 days patellas were used to study the chondrocyte metabolism by measuring the $\left.{ }^{35} \mathrm{~S}\right]$ sulphate incorporation.

Study 1: Two days postoperatively, in hyaluronan-treated cartilage the percentage of dead cells was $6.7 \%$, which was significantly lower compared to $16.2 \%$ in saline-treated cartilage. After three months the percentages of dead cells in both groups were statistically similar. Study 2: Hyaluronan treatment resulted in significantly higher $\left[{ }^{35} \mathrm{~S}\right]$ sulphate incorporation compared to knees irrigated with $0.9 \% \mathrm{NaCl}$.

These results suggest a potential role for hyaluronan in preventing cell death following articular cartilage injury. One injection of hyaluronan improved cartilage metabolism in knees with 6-month-old cartilage defects. 


\section{INTRODUCTION}

Hyaluronan (HYA) has received a great deal of attention as a potential agent of intervention in osteoarthritis. The results of clinical studies on the effect of HYA are equivocal. Significant improvements in pain, patient global assessment and functional outcomes with few adverse events were found due to the intraarticular injection of HYA, ${ }^{69,70}$ while others only found a small effect. ${ }^{71,72} \mathrm{HYA}$ is an important contributor to joint homeostasis and normally present in the joint's synovial fluid in high concentrations. ${ }^{193,}{ }^{194}$ HYA covers cartilage surfaces, is found in high concentrations in the direct vicinity of the chondrocyte, and has been reported to have anti-inflammatory, anabolic, analgesic and chondroprotective qualities. ${ }^{195}$

During arthroscopic procedures, joint irrigation results in dilution of synovial fluid with diminishing HYA concentrations. As a result, the physical characteristics and protective functions of the synovial fluid deteriorate, possibly leading to a higher vulnerability of the cartilage. In a clinical trial HYA was injected after an arthroscopic knee joint lavage, which resulted in reduction of pain and joint effusion together with an improvement in daily activities during the first 28 days. ${ }^{196}$ We propose that the mechanism behind these effects is improvement of joint homeostasis which in turn maintains cartilage metabolism through prevention of cell death. We further elaborate this theory.

In previous animal experiments we have shown that one injection with high molecular weight HYA restored the metabolism of chondrocytes that was inhibited by the irrigation solution. ${ }^{154,155}$ While these experiments were done in anatomically normal cartilage, in a clinical setting cartilage metabolism is often negatively influenced by cartilage lesions and a disturbed joint homeostasis. $^{25}$

First, we studied the effect of HYA on chondrocyte death surrounding a freshly prepared partial-thickness articular cartilage lesion as can occur during arthroscopic procedures. Secondly, the effect of HYA on chondrocyte metabolism in knee joints with a long-existing cartilage lesion was studied, better reflecting the clinical situation, when arthroscopy is first performed weeks to months after the original injury.

\section{Methods}

\section{Animals}

The experiments were conducted following the national and European guidelines for animal experiments. The Maastricht University committee for 
animal experiments approved all experiment protocols. A total of 23 6-monthold female New Zealand white (NZW) rabbits were used. Postoperative the rabbits were housed in a cage, after which they were allowed unlimited activity in groups in a stable. They were fed a standard rabbit diet with water ad libitum. During follow-up no joint immobilization was used.

\section{Hyaluronan}

The HYA in Ostenil ${ }^{\circledR}$ (Chemedica, Munich, Germany) has a mean molecular weight of $1.2 \mathrm{MDa}$. Ostenil ${ }^{\circledR}$ was diluted with a solution of $0.9 \% \mathrm{NaCl}$ to a concentration of $5 \mathrm{mg} / \mathrm{mL}$ HYA. ${ }^{155}$

\section{Surgical procedure of creating partial-thickness articular cartilage defects}

Preoperatively each rabbit was fasted for 12 hours. General anaesthesia was induced by intramuscular injection of $35 \mathrm{mg} / \mathrm{kg}$ body weight ketamine hydrochloride and $5 \mathrm{mg} / \mathrm{kg}$ xylazine and maintained throughout the surgical procedure by administration of $2 \%$ halothane and a mixture of oxygen and nitrous oxide delivered through an automatic ventilator using a specially designed mask. Preoperatively, all rabbits received an intramuscular injection of $10 \mathrm{mg} / \mathrm{kg}$ body weight ceftiofur (Pharmacia \& Upjohn, Woerden, The Netherlands) to reduce the risk of per- or postoperative infections. Postoperative pain killing was done by administering $50 \mu \mathrm{g} / \mathrm{kg}$ buphenomorphine at 2 hours and 1 day.

All operations were performed under strictly aseptic conditions. An arthrotomy of the tibia-femoral articulation was performed and the patella was dislocated laterally to expose the medial femoral condyle. A 4-mm diameter skin-biopsy punch (Kai Medical, Solingen, Germany) was used to circumscribe the defect centred on the weight-bearing part of the medial femoral condyle. Noncalcified cartilage was removed from the outlined defect using a scalpel. Special care was taken to prevent penetration of the subchondral bone.

\section{Study design}

The 23 NZW rabbits, that had received partial-thickness articular cartilage defects created on each medial femoral condyle, were divided in 2 groups. Fourteen rabbits were used to study the effect of HYA in preventing cell death peripheral to a partial-thickness articular cartilage defect (Study 1). The remaining 9 rabbits were used to study the effect of HYA on cartilage metabolism in joints with long-lasting partial-thickness articular cartilage defects (Study 2). 


\section{Study 1: Effect of HYA on cell death peripheral to a partial-thickness articular cartilage lesion}

Knee joints were rinsed with $0.9 \% \mathrm{NaCl}$, immediately after creating the cartilage lesion, and subsequently closed in layers. In the experimental knee $1 \mathrm{~mL}$ HYA solution was injected (HYA group); the contralateral knee received $1 \mathrm{~mL} 0.9 \%$ $\mathrm{NaCl}(\mathrm{NaCl}$ group). At two days following surgery 6 and at three months followup 8 animals were sacrificed. Condyles were fixated in a $10 \%$ formalin solution over 5 days at $4^{\circ} \mathrm{C}$. After decalcification in EDTA, samples were dehydrated in a series of increasing concentrations of ethanol and embedded in 2-hydroxyethyl methacrylate (Technovit ${ }^{\circledR} 7100$; Heraeus Kulzer, Wehrheim, Germany). Sections of $5 \mu \mathrm{m}$ were cut along the midsagittal plane using a microtome (LKB multirange microtome, Stockholm, Sweden), and stained with thionine. All sections were examined blindly to group assignment. A $50 \mu \mathrm{m}$ region of the articular cartilage adjacent to either side of the defect was examined using 200X and 400X magnification to assess peripheral chondrocyte death and viability. The percentage of cell death was measured as ratio between the number of dead cells and a total of at least 300 cells. Cell death was defined as the presence of a condensed, pycnotic nucleus and either a shrunken or deeply eosinophylic cytoplasm, or fragmentation of the nucleus/cytoplasm, or an empty lacuna. ${ }^{197-}$ ${ }^{199}$ The cell densities were determined in the sections of the 3 months follow-up samples by counting the number of living cells in an area of $50 \times 100 \mu \mathrm{m}$ alongside the defect. The cell density of the lateral condyle present on the same object glass served as a $100 \%$ control.

\section{Study 2: Effect of HYA on chondrocyte metabolism}

Six months after creating the partial-thickness articular cartilage defects, knee joints of 9 rabbits were treated again. An injection needle was placed into the knee joints, and i) were not irrigated (sham group, $n=6$ knees); or ii) were irrigated with $10 \mathrm{~mL} 0.9 \% \mathrm{NaCl}$ ( $\mathrm{NaCl}$ group, $n=6$ knees); or iii) received $1 \mathrm{~mL}$ HYA solution after irrigation with $10 \mathrm{~mL} 0.9 \% \mathrm{NaCl}$ (HYA group, $n=6$ knees). Seven days postoperatively, patellae were harvested to study the total sulphate incorporation rate (in dpm/patella) using a previously described method. ${ }^{200,} 201$ In brief, patellae were transferred to tubes containing $1 \mathrm{~mL}$ of medium (Dulbecco's Modified Eagle's Medium supplemented with 10\% fetal bovine serum (Invitrogen, Breda, The Netherlands), ascorbic acid 2-phosphate (0.2 mM) (Sigma-Aldrich Chemie B.V., Zwijndrecht, The Netherlands), penicillin (100 $\mathrm{U} / \mathrm{mL})$, streptomycin $(100 \mu \mathrm{g} / \mathrm{mL})$ and amphotericin B $(0.25 \mu \mathrm{g} / \mathrm{mL})$ (Invitrogen, Breda, The Netherlands). The medium was discarded and to each patella $1 \mathrm{~mL}$ medium supplemented with $5.7 \mu \mathrm{Ci} / \mathrm{mL} \mathrm{Na}_{2}{ }^{35} \mathrm{SO}_{4}\left({ }^{35} \mathrm{~S}\right]$ sulphate medium) 
(Amersham Biosciences Benelux, Roosendaal, The Netherlands) was added. The samples were incubated in a humidified $\mathrm{CO}_{2}$-incubator $\mathrm{O} / \mathrm{N}$. The $\left[{ }^{35} \mathrm{~S}\right]$ sulphate medium was removed and patellae were washed $2 \times 10$ minutes with $1 \mathrm{~mL}$ sterile phosphate-buffered saline. To the patella specimens $0.15 \mu \mathrm{g} / \mu \mathrm{L}$ proteinase $\mathrm{K}, 50 \mathrm{mM}$ Tris- $\mathrm{HCl}\left(\mathrm{pH}\right.$ 7.9) and $1 \mathrm{mM} \mathrm{CaCl}_{2}$ were added, and subsequently patellae were left in a shaking water bath at $56^{\circ} \mathrm{C} \mathrm{O} / \mathrm{N}$. After centrifugation at 3,000 g for 5 minutes, supernatants were supplemented with $25 \mu \mathrm{L} 0.1 \%$ cetylpyridinium chloride (Merck-Europe B.V., Amsterdam, The Netherlands) (CPC) solution and $25 \mu \mathrm{L} 4 \mathrm{mg} / \mathrm{mL}$ proteoglycan solution (A1 fraction, isolated from human articular cartilage). Samples were incubated at $37^{\circ} \mathrm{C}$ for 1 hour to precipitate the glycosaminoglycans, which were then centrifuged at 15,000 g for 5 minutes. The supernatants were discarded and the pellets were washed once with $100 \mu \mathrm{L}$ of a solution of $0.1 \% \mathrm{CPC}$ in $0.2 \mathrm{M} \mathrm{NaCl}$ and then dried. Pellets were dissolved in formic acid (Merck-Europe B.V., Amsterdam, The Netherlands) at room temperature $\mathrm{O} / \mathrm{N}$. A $20 \mu \mathrm{L}$ aliquot of each sample was mixed with $2 \mathrm{~mL}$ Formula 989 scintillation fluid (DuPont, Dordrecht, The Netherlands) and counted in a liquid scintillation counter. The total sulphate incorporation rate of each sample was calculated using the specific activity of the medium.

\section{Statistical analysis}

Differences were tested for significance through non-parametric statistical tests. The Wilcoxon rank sum test for paired-samples was applied in the case of intraanimal comparisons to compare percentages of cell death for each follow-up separately (Study 1); and to evaluate the effect of HYA on chondrocyte metabolism (Study 2). The Mann-Whitney $U$-test for independent samples was applied when comparing the results obtained from different animals to evaluate the statistical difference in the percentage of dead cells between 2 days and 3 months follow-up (Study 1). The Mann-Whitney $U$-test was applied when comparing the cell densities of living cells between the HYA and $\mathrm{NaCl}$ groups. All statistical analyses were performed by a statistician (NG) using SPSS-pc, version 12.0.1 (SPSS Inc., Chicago). An alpha level of 0.05 was chosen to judge statistical significance.

\section{Results}

\section{Study 1: Effect of HYA on cell death peripheral to a partial-thickness articular cartilage lesion}

In the postoperative period, no limp or swollen knee joints were observed in any of the rabbits. No complications such as infection or patellar luxations occurred. 


\section{HYA prevents cell death and improves cell metabolism}

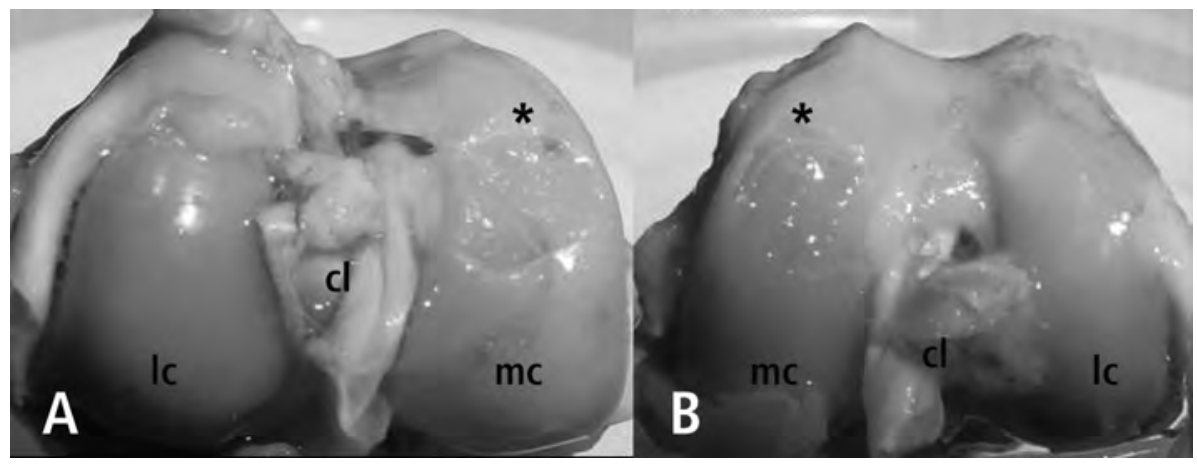

Figure 1. Representative photographs are shown from articular surfaces 2 days after creating partial-thickness articular cartilage defects $\left(^{*}\right)$ on rabbit medial femoral condyles. (A) injected with hyaluronan after irrigation with $0.9 \% \mathrm{NaCl}$ immediately after creating the defects, or (B) irrigated with $0.9 \% \mathrm{NaCl}$. Note that in both hyaluronan- and $\mathrm{NaCl}$-treated knees the surface is smooth and glossy without gross osteoarthritic features. Abbreviations: mc, medial femoral condyle; Ic, lateral femoral condyle; $\mathrm{Cl}$, cruciate ligaments. (for full-colour figure see page 198)

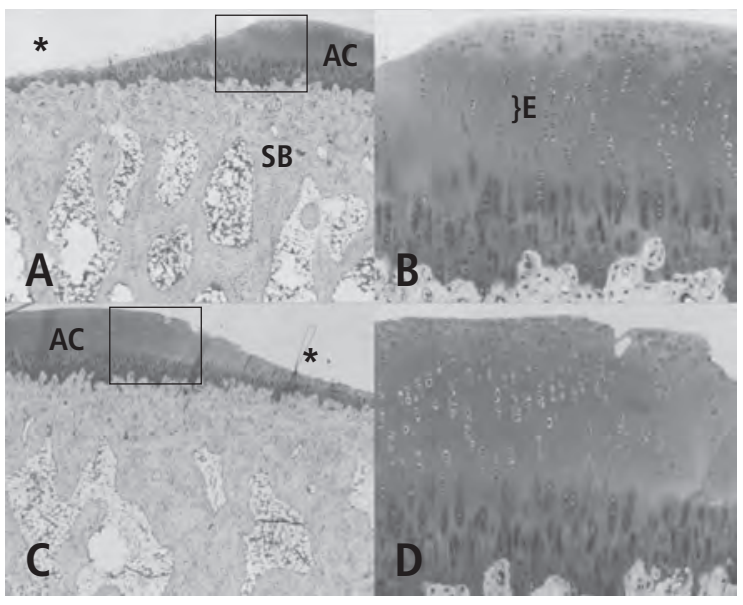

Figure 2. These micrographs represent thionine-stained sections of articular cartilage at the periphery of partial-thickness articular cartilage defects $\left({ }^{*}\right)$ created on rabbit medial femoral condyles which were immediately treated with hyaluronan or $0.9 \% \mathrm{NaCl}$ solution. Animals were sacrificed 2 days postoperatively. Note defects have not penetrated the subchondral bone. (A) irrigated with $0.9 \% \mathrm{NaCl}$; (B) is an enlargement of the box in (A). (C) injected with hyaluronan after irrigation with $0.9 \% \mathrm{NaCl}$; (D) is an enlargement of the box in (C). Note the more empty lacunae $(\mathrm{E})$ can be detected in area surrounding the lesion after $0.9 \% \mathrm{NaCl}$ irrigation only. Original magnifications (A and B) 50X, (C and D) 200X. Abbreviations: AC, articular cartilage; SB, subchondral bone; $\mathrm{E}$, empty lacunae indicative for dead chondrocytes. 
Macroscopic impressions of HYA- and $\mathrm{NaCl}$-treated knees were similar, with smooth and glossy cartilage that surrounded the defect (Figure 1). Gross osteoarthritic features, like osteophytes and other joint deformities, were not observed. Lesions were centred on the weight-bearing part of the medial femoral condyle, and were not healed at 2 days or 3 months follow-up.

Histologic analyses confirmed the macroscopic observation that none of the partial-thickness articular cartilage defects was healed at 2 days (Figure 2) or 3 months (Figure 3 ) after surgery. Defects were up to the calcified cartilage without penetrating the subchondral bone (Figure 2A, C; Figure $3 \mathrm{~A}, \mathrm{C}$ ), indicating that true partial-thickness articular cartilage defects were created. Some defects were covered with debris, which never filled the defects completely. Three months post-surgery, the cartilage peripheral to the defects showed degenerative features like surface irregularities, cluster formation and loss of proteoglycan staining (Figure 3), irrespective of the applied treatment. Signs of synovial tissue inflammation were absent.

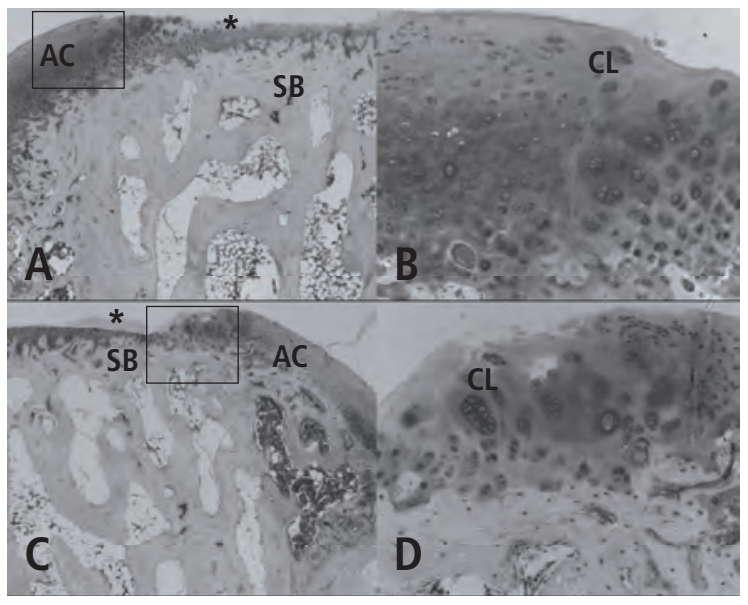

Figure 3. These micrographs represent thionine-stained sections of articular cartilage at the periphery of partial-thickness articular cartilage defects $\left(^{*}\right)$ created on rabbit medial femoral condyles which were immediately treated with hyaluronan or $0.9 \% \mathrm{NaCl}$ solution. Animals were sacrificed 3 months postoperatively. Note that none of the defects was healed. (A) irrigated with $0.9 \% \mathrm{NaCl}$; (B) is an enlargement of the box in (A). (C) injected with hyaluronan after irrigation with $0.9 \% \mathrm{NaCl}$; (D) is an enlargement of the box in (C). Note the similar features concerning cluster $(\mathrm{CL})$ formation and loss of proteoglycan staining in cartilage from hyaluronan-treated and $0.9 \% \mathrm{NaCl}$-treated knees. Original magnification, (A and B) 50X, (C and D) 200X. Abbreviations: $\mathrm{AC}$, articular cartilage; $\mathrm{SB}$, subchondral bone; $\mathrm{CL}$, cell cluster. 
At 200X and 400X magnification we judged whether chondrocytes were alive or dead. Two days after creating a partial-thickness cartilage lesion, the percentage of dead cells in the periphery of the defect in the group treated with HYA was significantly lower $(p=0.028)$ as compared to the $\mathrm{NaCl}$ group: $6.7 \%$ \pm 4.1 versus $16.2 \% \pm 4.6$, respectively (Table $1 \mathrm{~A}$; Figure 4 ). At 3 months followup the percentage of dead cells did not differ significantly $(p=0.327)$ between the $\mathrm{NaCl}$ and the HYA group: $6.1 \% \pm 4.1$ versus $9.5 \% \pm 8.1$, respectively (Table $1 \mathrm{~B}$; Figure 4). In the $\mathrm{NaCl}$ group the percentage of dead cells was significantly lower after 3 months compared to 2 days after surgery $(p=0.004)$. In HYAtreated knees, the percentage of dead cells remained statistically similar over the course of 3 months $(p=0.747)$.

At three months follow-up the cell densities of the cartilage alongside the defects varied from $10-120 \%$ of the control, due to either hypocellularity or the

Table 1. Number of living and dead cells counted at the periphery of partial-thickness articular cartilage defects.

A. 2 days postinjury

\begin{tabular}{cccc}
\multicolumn{2}{c}{ NaCl-treated knees } & \multicolumn{2}{c}{ Hyaluronan-treated knees } \\
\hline Living cells & Dead cells & Living cells & Dead cells \\
\hline 300 & 38 & 300 & 4 \\
307 & 56 & 300 & 20 \\
341 & 52 & 301 & 18 \\
317 & 31 & 313 & 12 \\
316 & 61 & 315 & 28 \\
310 & 69 & 302 & 40 \\
\hline
\end{tabular}

B. 3 months postinjury

$\mathrm{NaCl}$-treated knees

Hyaluronan-treated knees

\begin{tabular}{cccc}
\hline Living cells & Dead cells & Living cells & Dead cells \\
\hline 328 & 7 & 335 & 6 \\
311 & 20 & 323 & 69 \\
300 & 4 & 320 & 13 \\
316 & 13 & 338 & 15 \\
349 & 39 & 327 & 59 \\
350 & 12 & 250 & 6 \\
351 & 25 & 305 & 54 \\
314 & 40 & 303 & 18 \\
\hline
\end{tabular}




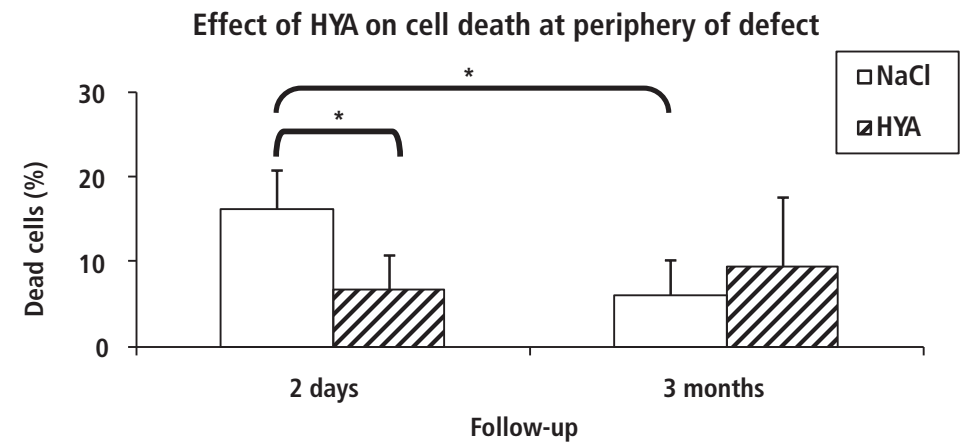

Figure 4. This histogram represents the mean percentages of dead chondrocytes. Partial-thickness articular cartilage defects were created and rinsed with $0.9 \% \mathrm{NaCl}$. Experimental knees were immediately injected with hyaluronan; control knees with $0.9 \% \mathrm{NaCl}$. Follow-up was 2 days (HYA, $n=6$ knees; $\mathrm{NaCl}, n=6$ knees) and 3 months (HYA, $n=8$ knees; $\mathrm{NaCl}, n=8$ knees). Error bars designate means plus standard deviation.

Abbreviations: *, $p<0.05$; HYA, hyaluronan

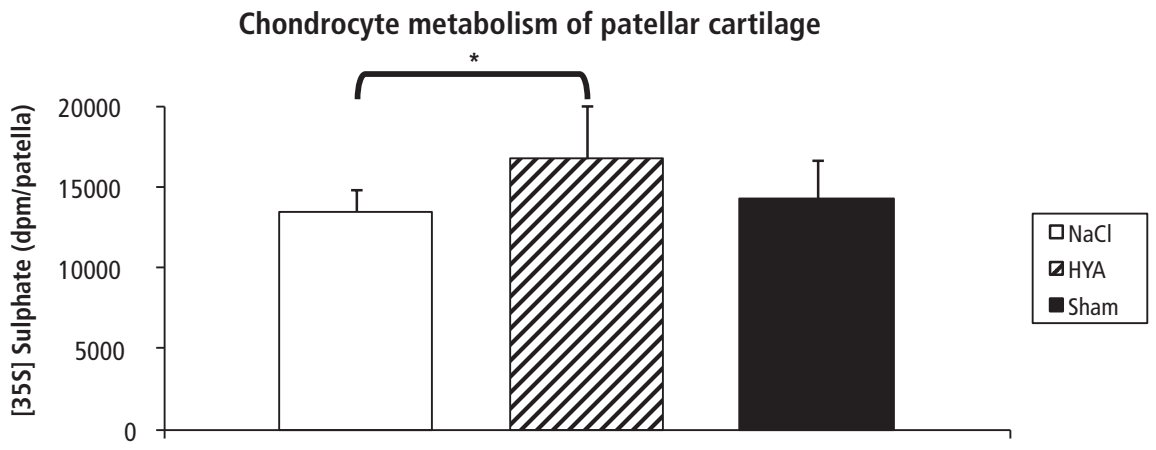

Figure 5. This histogram represents the mean $\left.{ }^{35} \mathrm{~S}\right]$ sulphate incorporation, expressed in $\mathrm{dpm} /$ patella. Knee joints were i) left untreated i.e. no irrigation, no treatment (Sham, $n=6$ knees); or after 6 months ii) irrigated with $0.9 \% \mathrm{NaCl}(\mathrm{NaCl}, n=6$ knees); or iii) injected with hyaluronan solution after irrigation with $0.9 \% \mathrm{NaCl}$ (HYA, $n=6$ knees). Cartilage metabolism was assessed on the patella ex vivo 7 days after the treatment.

Error bars designate means plus standard deviation.

Abbreviations: *, $p<0.05$; HYA, hyaluronan 
presence of cell clusters, respectively. Cell densities of the HYA-treated and the $\mathrm{NaCl}$-treated cartilage samples did not differ significantly: $53.7 \%$ (range, $10-120$ ) compared to $73.3 \%$ (range, $44-114$ ) for the HYA and $\mathrm{NaCl}$ group, respectively.

\section{Study 2: Effect of HYA on chondrocyte metabolism}

The average incorporated $\left[{ }^{35} \mathrm{~S}\right]$ sulphate $(\mathrm{dpm} /$ patella) in glycosaminoglycans of the HYA-treated group was significantly higher $(p=0.029)$ compared to knee joints that were not treated with $\mathrm{HYA}$ after $0.9 \% \mathrm{NaCl}$ irrigation ( $\mathrm{NaCl}$ group): $16,751( \pm 3,261)$ versus $13,503( \pm 1,329)$ for HYA- and NaCl-treated knees, respectively (Figure 5). The incorporated $\left[{ }^{35} \mathrm{~S}\right]$ sulphate in glycosaminoglycans of HYA-treated knees was slightly, but not significantly, higher than in shamtreated knees: $16,751( \pm 3,261)$ versus $14,283( \pm 2,368)$ for HYA- and shamtreated knees, respectively.

Irrigation of previously injured rabbit joints with $\mathrm{NaCl}$ solution inhibited cartilage metabolism, as was shown by a lower ${ }^{35}$ S] sulphate incorporation into the glycosaminoglycans as compared to the sham-treated knees: $13,503( \pm 1,329)$ versus $14,283( \pm 2,368)$ for $\mathrm{NaCl}$ - and sham-treated knees, respectively. Differences between the $\mathrm{NaCl}$ - and sham-treated knees were not statistically significant.

\section{Discussion}

This in vivo study shows the effect on chondrocyte death and chondrocyte metabolism after one intra-articular injection of HYA in the injured rabbit knee.

It is known from literature that chondrocyte death occurs close to a lesion in response to experimental mechanical compression and cartilage injury. ${ }^{14}$ The earliest signs of apoptosis appear around 6 hours post-injury and the percentage of apoptotic cells increase up to 7 days after injury. ${ }^{24}$ In the present study we evaluated cell death after one intra-articular injection with HYA, which was injected immediately after creating a partial-thickness articular cartilage defect. Treatment with HYA resulted in the protection of chondrocytes peripheral to the cartilage defect, whereas a relatively high percentage of dead cells was observed in untreated knees 2 days after creating the defect. This is in agreement with the findings of Díaz-Gallego et al., ${ }^{202}$ who pointed out that intra-articular treatment with HYA exerts a protective role in cartilage, reducing apoptosis when treatment is started early. These findings support the theory of a therapeutic window during which apoptosis may be inhibited by therapeutic agents, ${ }^{203}$ in which HYA likely exerts its protective effect by HYA-induced reduction of antiFas-induced chondrocyte apoptosis. ${ }^{204}$ It remained unclear as to whether HYA is chondroprotective in the long-term. In the current study the percentage of dead 
chondrocytes in HYA-treated knees did not increase between 2 days and 13 weeks postoperatively. Unexpectedly, in untreated knees the percentage of dead chondrocytes decreased over the course of 13 weeks, showing comparable percentages of cell death as those seen in the HYA-treated knees. This most likely is due to removal of the dead cells, which will ultimately result in a lower cell density of the cartilage. However, assessment of the cell densities did not provide an answer, due to the high variability between the samples caused by both hypocellular regions and regions with cell clusters. During the occurring degeneration the empty cell lacunae then were removed or masked, resulting in a too low number of dead cells. Our histology was not conclusive at this point. Finding no convincing evidence for a protective role of HYA on the long term corroborates with data of Mendelson et al. ${ }^{205}$ Weekly HYA injections for 3 weeks, starting at either 1 or 3 weeks following injury, did not provide protection to zones peripheral to partial-thickness articular cartilage lesions at either 2 or 6 months. In the latter study the timing of initiation of the injection differed from our study. This could be an important factor concerning the therapeutic window in which HYA could exert its beneficial effect.

Knee joints having 6-month-old partial-thickness articular cartilage defects were irrigated with $\mathrm{NaCl}$ and subsequently treated with one intra-articular HYA injection. Seven days later chondrocyte metabolism was improved in these injured knees as compared to saline-treated controls. We used a previously described model in which cartilage degeneration was induced by wellcircumscribed, surgically-created partial-thickness articular cartilage defects to reflect the clinical situation more accurately. ${ }^{206}$ In order to measure the effect of HYA in knee joints with degenerative changes, cartilage from a primary noninjured part of the knee was used. Using this method, the lesion area was avoided. Analysis of the patella is suggested to reflect the metabolism in a damaged joint more accurately. Chondrocyte metabolism was studied through measuring the $\left[{ }^{35} \mathrm{~S}\right]$ sulphate incorporation into the glycosaminoglycans of whole patellas. This method was found as reliable and has been described by De Vries et al. ${ }^{200}$ It has been shown that $98 \%$ of the radiolabel is taken up by the cartilage of the patella and only $2 \%$ by bone, making it unnecessary to measure these separately. We have shown previously that joint irrigation caused a disturbed chondrocyte metabolism in anatomically healthy knee joints: After $\mathrm{NaCl}$ irrigation, chondrocyte metabolism in patellas was inhibited for 7 days after the procedure. ${ }^{207}$ However, one injection of $5 \mathrm{mg} / \mathrm{mL}$ HYA was able to restore the by $\mathrm{NaCl}$ disturbed cartilage metabolism completely to normal. ${ }^{208}$ The current study showed that also in injured knees HYA can restore the impaired chondrocyte metabolism, caused by the irrigation procedure. 
In summary, HYA has a chondroprotective effect on the short-term when applied immediately post-injury, and improves chondrocyte metabolism in knee joints with long-existing lesions. However, the effect of HYA on the longterm, and the relationship between cell death and cell metabolism remains unclear. Future studies are designed to show a possible relationship between prevention of cell death due to HYA and improved cell metabolism in knee joints with long-existing articular cartilage lesions. 


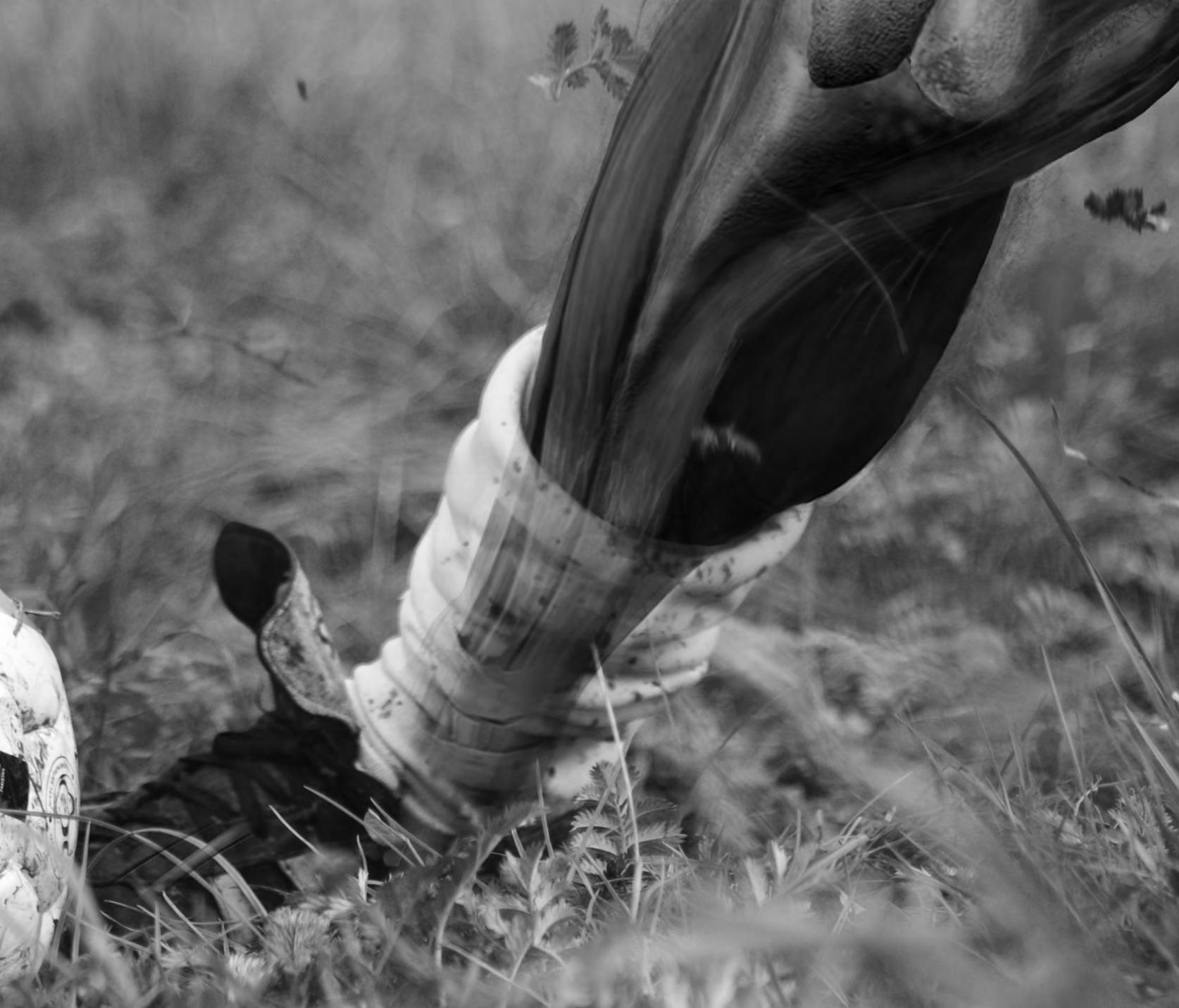




\section{CHAPTER 5}

\section{PEOT/PBT based scaffolds with}

low mechanical properties improve cartilage repair tissue formation in osteochondral defects

E.J.P. Jansen, J. Pieper, M.J.J. Gijbels, N.A. Guldemond, J. Riesle, L.W. Van Rhijn, S.K. Bulstra, R. Kuijer

Journal of Biomedical Materials Research Part A. 2008 Apr 22. 


\section{Abstract}

The aim of our study was to compare the healing response of biomechanically and biochemically different scaffolds in osteochondral defects created in rabbit medial femoral condyles.

A block copolymer comprised of poly(ethylene oxide terephthalate) and poly(butylene terephthalate) was used to prepare porous scaffolds. The 70/30 scaffold (70 weight $\%$ poly(ethylene oxide terephthalate)) was compared to the stiffer 55/45 (55 weight\% poly(ethylene oxide terephthalate)) scaffold. Nine 6month-old rabbits were used. Osteochondral defects were filled with 55/45 scaffolds $(n=6) ; 70 / 30$ scaffolds $(n=6)$; or left empty $(n=6)$. Defect sites were allowed to heal for 12 weeks. Condyles were macroscopically evaluated and analyzed histologically using the O'Driscoll score for evaluating repair of osteochondral defects.

Repair tissue in 70/30 scaffolds consisted of cartilage-like tissue on top of trabecular bone, whereas the tissue within the 55/45 scaffolds consisted predominantly of trabecular bone. O'Driscoll scores for 70/30 scaffolds were significantly better $(p=0.024)$ in comparison to untreated osteochondral defects and 55/45 scaffolds.

This study reveals that the biomechanical and biochemical properties of the scaffold play an important role by themselves, and can affect the healing response of osteochondral defects. Scaffolds with low mechanical properties were superior in cartilage repair tissue formation. 


\section{INTRODUCTION}

Articular cartilage lesions fail to heal spontaneously and even may evolve to osteoarthritis (OA). ${ }^{209,} 210$ While partial-thickness articular cartilage lesions do not heal, some spontaneous repair is seen in osteochondral defects (OCDs). However, the resulting fibrocartilage lacks the biochemical and structural characteristics of articular cartilage, and usually undergoes degeneration within 6 to 12 months. ${ }^{10,211-213}$

Clinically, the prevention of degeneration in the affected joint is an important rationale for repairing these cartilage defects. Unfortunately, the current therapeutic strategies do not predictably restore a durable articular surface, and have not yet been proven to be efficacious in preventing OA. Therefore, research has been focused on repair of articular surfaces by tissue engineering applications using three-dimensional synthetic porous scaffolds. ${ }^{214}$ They serve as a scaffolding for the expansion and differentiation of transplanted cells within the defect space. Also scaffolds in the absence of seeded cells (cellfree approach) have been applied..$^{215-218}$ The principal advantage to be derived from filling a large defect with a scaffold is that the critical-size limit for a spontaneous healing response can be overcome by its bridging action. ${ }^{219}$ The scaffold is infiltrated with blood and bone-marrow-derived material from the damaged subchondral bone and bone-marrow spaces which form a granulation tissue. The local conditions pertaining within the defect space will likely promote transformation of repair tissue into cartilage. ${ }^{219}$ At present, however, repair results have been inconsistent with respect to cartilage and subchondral bone formation, in particular at long-term follow-up.

Several factors may contribute to this variable healing outcome. The choice of scaffold material might be a critical determinant. It has to provide a porous biocompatible network into which surrounding tissue is induced and acts as a temporary template for the new tissues growth and organization. Also, the scaffolds should be permeable to permit the ingress of cells and nutrients and elution of waste products, and should exhibit the appropriate surface chemistry for cell attachment. ${ }^{135}$ Not only the composition, but also the mechanical properties of the scaffold are important. Scaffolds have to withstand physiological loading such that the strength of the scaffold is retained until the regeneration tissue can assume its structural role.

Biocompatible and biodegradable poly(ethylene oxide terephthalate)/ poly(butylene terephthalate) (PEOT/PBT) block copolymers were extensively 
tested. ${ }^{156-159}$ However, the in vivo healing response of OCDs using PEOT/PBT based porous scaffolds is unknown.

The aim of our study was to compare the in vivo performance of various PEOT/PBT scaffolds, which contained different physico-chemical and biomechanical properties. For this purpose, we evaluated and compared the in vivo healing response of OCDs in medial femoral condyles of New Zealand white (NZW) rabbits that were filled with cell-free PEOT/PBT scaffolds. We hypothesized that the stiff scaffolds, which most closely match the biomechanical properties of native articular cartilage, would improve the performance of tissue engineered cartilage.

\section{MATERIALS AND METHODS}

\section{Materials}

PEOT/PBT based scaffolds (4 millimetres in diameter $\times 4$ millimetres in length) were obtained from IsoTisOrthoBiologics (Bilthoven, The Netherlands). The polyethylene oxide (PEO) segment is hydrophilic and provides soft elastomeric properties, whereas poly(butylene terephthalate) (PBT) imparts stiffness to the system. During polymer synthesis, the molecular weight of PEO and weight ratio of the PEOT/PBT components can be defined to allow the copolymer to be tailored for desired mechanical and surface properties. ${ }^{220}$ In this study 2 cylindrical scaffolds were used: 70/30 scaffolds (weight $\%$ PEOT, 70; weight $\%$ PBT, 30) and 55/45 scaffolds (weight\% PEOT, 55; weight\% PBT, 45). The molecular weight in $\mathrm{g} / \mathrm{mol}$ of the starting PEG blocks used in the copolymerization was 1000 and 300 $\mathrm{g} / \mathrm{mol}$ for $70 / 30$ and $55 / 45$, respectively. The physico-chemical characterizations of the 55/45 and 70/30 scaffolds ${ }^{161,221}$ are provided in Table 1.

\section{Scaffold preparation}

The PEOT/PBT block copolymer was moulded and subsequently combined with $\mathrm{NaCl}$ grain in the volume ratio copolymer: $\mathrm{NaCl}=25: 75$ (salt weight fraction, $75 \%$ ). The salt particles were sieved to the desired particle size range 400-600 $\mu \mathrm{m}$. The mixture of the copolymer and the salt particles was cast into a stainless steel mould $\left(16 \times 16 \mathrm{~cm}^{2}\right)$ and brought under pressure (150 PSI). The mould was heated for 10 minutes at $210^{\circ} \mathrm{C}$. After cooling to room temperature (RT), the copolymer/salt composite was leached out of the membrane by immersing in demineralized water for several hours. The water was changed several times until all the salt was leached out, which was tested by conductivity $(<25 \mu \mathrm{S})$. The resulting scaffolds were air dried at RT for 48 hours, and in a vacuum oven at $50^{\circ} \mathrm{C}$ for 16 hours, and subsequently stored under 
Table 1. Physico-chemical characteristics of 55/45 and 70/30 scaffolds.

\begin{tabular}{|c|c|c|c|c|c|c|c|}
\hline $\begin{array}{l}\text { Copolymer } \\
\text { composition }\end{array}$ & $\begin{array}{l}\text { Porosity } \\
(\%)^{c}\end{array}$ & $\begin{array}{l}\text { Average } \\
\text { Pore Size } \\
(\mu \mathrm{m})^{c, d}\end{array}$ & $\begin{array}{l}\text { Pore Size } \\
\text { Range } \\
(\mu \mathrm{m})^{c, d}\end{array}$ & $\begin{array}{l}\text { Water-uptake } \\
(\%)^{e, f}\end{array}$ & $\begin{array}{l}\text { Degradation } \\
\text { expressed as } \\
\text { decrease in } \\
\text { Mw }(\%)^{e, g}\end{array}$ & $\begin{array}{l}\text { Equilibrium } \\
\text { Modulus } \\
(\mathrm{MPa})^{c}\end{array}$ & $\begin{array}{l}\text { Dynamic } \\
\text { Stiffness at } \\
0.1 \mathrm{~Hz}(\mathrm{MPa})\end{array}$ \\
\hline $55 / 45^{a}$ & $75.6 \pm 1.9$ & 182 & $6-450$ & $260 \pm 10$ & $34 \pm 8$ & $0.93 \pm 0.31$ & $1.72 \pm 0.33$ \\
\hline $70 / 30^{b}$ & $76 \pm 2$ & 160 & 6-455 & $520 \pm 10$ & $90 \pm 3$ & $0.18 \pm 0.001$ & $0.17 \pm 0.02$ \\
\hline
\end{tabular}

a 55/45; weight\% PEOT, 55; weight\% PBT, 45.

b 70/30; weight\% PEOT, 70; weight\% PBT, 30.

c Derived from Miot et al. ${ }^{161}$

d Measured using $\mu \mathrm{CT}$ analysis. ${ }^{161}$

e Derived from Lamme et al.221

f Water-uptake (wt\%) is expressed as $\left(m-m_{0}\right) / m_{0}(n=6 \pm S D){ }^{221}$

$g$ Decrease in molecular weight is measured using GPC following subcutaneous implantation of scaffolds in mini-pigs for 52 weeks and expressed as percentage of Mw before implantation $\left(n=3 \pm\right.$ SD). ${ }^{221}$

vacuum conditions. One day prior to implantation scaffolds were sterilized by $\gamma$ irradiation (25 kGy) and pre-wetted in phosphate-buffered saline (PBS).

\section{Animal care}

Dutch and European guidelines for the care and use of laboratory animals have been observed. The Maastricht University committee for animal experiments approved all rabbit experiment protocols. Preoperatively, each rabbit was fasted for 12 hours. General anaesthesia was induced by intramuscular injection of 35 $\mathrm{mg} / \mathrm{kg}$ body weight ketamine hydrochloride and $5 \mathrm{mg} / \mathrm{kg}$ xylazine and maintained throughout the surgical procedure by administration of $2 \%$ halothane and a mixture of oxygen and nitrous oxide delivered by an automatic ventilator using a specially designed mask. Preoperatively, all rabbits received an intramuscular injection of $10 \mathrm{mg} / \mathrm{kg}$ body weight ceftiofur (Pharmacia \& Upjohn, Woerden, The Netherlands) to reduce the risk of per- or postoperative infections. Postoperative pain killing was done by administering $50 \mu \mathrm{g} / \mathrm{kg}$ buphenomorphine at 2 hours and 1 day. Postoperatively, the rabbits were housed in a cage for 2 days, after which they were allowed unrestricted activity in groups in a stable. They were fed a standard rabbit diet and had water ad libitum. During follow-up no joint immobilization was used. All rabbits were euthanized at a follow-up of 3 months with an overdose of pentobarbital. 


\section{Experimental design}

Based on pilot work and using the power analysis of Sachs, with a power of $80 \%$, two-tailed and a confidence interval of $95 \%$, we came to a minimum of 6 knees per group. A total of nine 6-month-old female NZW rabbits, weighing between 3.2 and $4.4 \mathrm{~kg}$, were used. Each knee $(n=18)$ received one OCD on the medial femoral condyle. OCDs were randomly treated with a 55/45 scaffold $(n=6) ;$ a $70 / 30$ scaffold $(n=6)$; or left empty $(n=6)$. Defect sites were allowed to heal for 12 weeks, fully weight bearing. We evaluated and compared the in vivo healing response macroscopically and histologically.

\section{Surgical technique}

An arthrotomy of the tibia-femoral articulation was performed through a medial longitudinal parapatellar incision. The medial capsule was incised and the patella was dislocated laterally to expose the surface of the medial femoral condyle. A 4-mm width 4-mm deep cylindrical OCD was created using an electric drill and a pointed drill bit in which the depth of penetration was limited by a blunt ring. The drill was used at low speed under a continuous stream of $0.9 \mathrm{wt} \%$ saline solution $\left(4^{\circ} \mathrm{C}\right)$ to minimize the risk of heat necrosis. The PEOT/PBT scaffolds were press fitted into the resulting OCDs matching the height of the adjacent cartilage or left empty. The patellae were repositioned and the fasciae were closed with Vicryl ${ }^{\circledR} 2-0$. Much care was taken to approximate the medial capsule and extensor aperture to avoid patella luxation. The skin was closed with Vicry ${ }^{\circledR} 4-0$.

\section{Macroscopical and histological analyses}

At euthanasia, after 3 months, the femoral condyles were dissected and photographed for macroscopical analysis purposes. Condyles were fixated in a $10 \%$ formalin solution over 5 days at $4^{\circ} \mathrm{C}$. Following fixation, condyles were decalcified. Decalcification endpoint was determined using radiographs. Samples were dehydrated through a series of increasing concentrations of ethanol and embedded in 2-hydroxyethyl methacrylate (Technovit ${ }^{\circledR}$ 7100; Heraeus Kulzer GmbH\&Co., Wehrheim, Germany). Sections with thicknesses of $4 \mu \mathrm{m}$ were cut along the midsagittal plane (LKB multirange microtome, Stockholm, Sweden) and stained with thionine. Sections taken near the center of the defect were evaluated using light microscopy, and were examined blindly to group assignment by an experimental pathologist (MG) and orthopaedic resident (EJ). Each sample was graded by using a histological score for OCDs repair (maximum score, 27), which was described by O'Driscoll et al. ${ }^{222}$ (Table 2). 


\section{PEOT/PBT based scaffolds improve cartilage repair}

Table 2. O'Driscolls histological score for repair of osteochondral defects

Nature of predominant tissue

Hyaline articular cartilage

4

Incompletely differentiated mesenchyme or fibrocartilage

2

Fibrous tissue or bone

\section{Matrix staining}

Normal or nearly normal

3

Moderate

Slight

None

Structural characteristics

Surface regularity

Smooth and intact

3

Superficial horizontal lamination

Fissures $25-100 \%$ of thickness

Structural integrity

Normal or nearly normal

2

Slight disruption including cysts

Severe desintegration

\section{Filling of lesion}

$>110 \%$
$90-110 \%$
$50-90 \%$
$<50 \%$

Bonding to adjacent cartilage

Bonded to both ends of graft

Bonded to one end of graft

Not bonded

\section{Subchondral bone}

Good

Under level or too high

Filled with cartilage

None or far too high

1

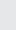




\begin{tabular}{lc} 
Freedom from cellular changes of degeneration & \\
Hypocellularity & 3 \\
\hline Normal cellularity & 2 \\
Slight hypocellular & 1 \\
Moderate hypocellular or hypercellular & 0 \\
Severe hypocellular or no chondrocytes & 2 \\
\hline Chondrocyte clustering & 1 \\
\hline No clusters & 0 \\
$<25 \%$ of the cells & \\
$25-100 \%$ of the cells or no chondrocytes & 3 \\
\hline Freedom from degenerative changes in adjacent cartilage & 2 \\
\hline Normal cellularity, no clusters, normal staining & 1 \\
Normal cellularity, mild clusters, moderate staining & 0 \\
Mild or moderate hypocellularity, slight staining &
\end{tabular}

\section{Statistics}

Data were not normally distributed and therefore were analyzed using nonparametric tests. Then, a two-tailed Mann-Whitney $U$ test was performed to compare differences in O'Driscoll score between non-, 70/30-, and 55/45treated OCDs. A $p$ value less than 0.05 was considered significant. All statistical analyses were performed by a statistician (NG) using SPSS Version 12.0.1 (SPSS Inc, Chicago, IL).

\section{Results}

\section{Animals}

The rabbits limped for the first days, but bore full weight on the operated limbs when allowed activity. A swollen and warm knee was observed in one rabbit during the first 2 days postoperatively, which disappeared spontaneously later on. Patella luxations were not observed.

\section{Macroscopic observations}

Eighteen knees (55/45, $n=6$; 70/30, $n=6$; untreated, $n=6$ ) were harvested and used for further examination. Figure 1 shows representative photographs of femoral condyles harvested at 3 months follow-up. All OCDs were completely filled and showed nice congruency with the surrounding articular cartilage. 

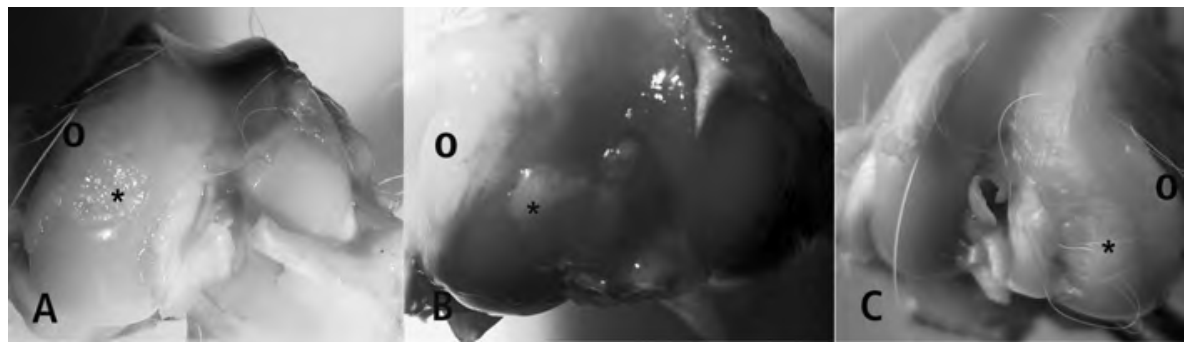

Figure 1. Representative photographs are shown of rabbit knees 3 months after creating osteochondral defects $\left(^{*}\right)$ in medial femoral condyles. Osteochondral defects were left empty (A); or were filled immediately with 55/45 (B) or 70/30 (C) scaffolds. Note the osteophytes (o) on the ridge of femoral condyles. (for full-colour figure see page 199)

They were distinguishable from the adjacent native articular cartilage by their glossy white appearance. All OCDs had a smooth surface with palpation. Osteophytes were observed in all untreated, 55/45 and 70/30 treated knees.

\section{Histologic analyses}

Table 3 shows the medians of untreated, 55/45 and 70/30 treated OCDs using the subcategories of the O'Driscoll score.

Table 3. Scores for repair of osteochondral defects using O'Driscolls subcategories

\begin{tabular}{|c|c|c|c|c|c|c|}
\hline \multirow[t]{2}{*}{ Subcategories O'Driscoll score } & \multicolumn{2}{|c|}{ Untreated } & \multicolumn{2}{|c|}{$55 / 45$} & \multicolumn{2}{|c|}{$70 / 30$} \\
\hline & median & range & median & range & median & range \\
\hline Predominant tissue & 2.0 & $0.0-2.0$ & 1.0 & $0.0-2.0$ & 2.0 & $2.0-2.0$ \\
\hline Matrix staining & 1.0 & $0.0-2.0$ & 0.0 & $0.0-2.0$ & 3.0 & $2.0-3.0$ \\
\hline Surface & 1.0 & $1.0-3.0$ & 2.0 & $1.0-3.0$ & 1.0 & $1.0-3.0$ \\
\hline Integrity & 0.0 & $0.0-0.0$ & 0.0 & $0.0-0.0$ & 0.0 & $0.0-1.0$ \\
\hline Filling & 2.0 & $1.0-2.0$ & 2.0 & $1.0-2.0$ & 2.0 & $1.0-2.0$ \\
\hline Bonding & 1.0 & $1.0-2.0$ & 2.0 & $1.0-2.0$ & 2.0 & $1.0-2.0$ \\
\hline Subchondral bone & 2.0 & $0.0-2.0$ & 3.0 & $1.0-3.0$ & 2.0 & $2.0-2.0$ \\
\hline Hypocellularity & 1.5 & $0.0-3.0$ & 0.0 & $0.0-3.0$ & 3.0 & $3.0-3.0$ \\
\hline Clustering & 1.0 & $0.0-2.0$ & 0.0 & $0.0-1.0$ & 1.0 & $1.0-2.0$ \\
\hline Degenerative changes & 0.0 & $0.0-3.0$ & 1.5 & $0.0-2.0$ & 1.0 & $0.0-3.0$ \\
\hline Total O'Driscoll score & 11.5 & & 11.5 & & 17.0 & \\
\hline
\end{tabular}




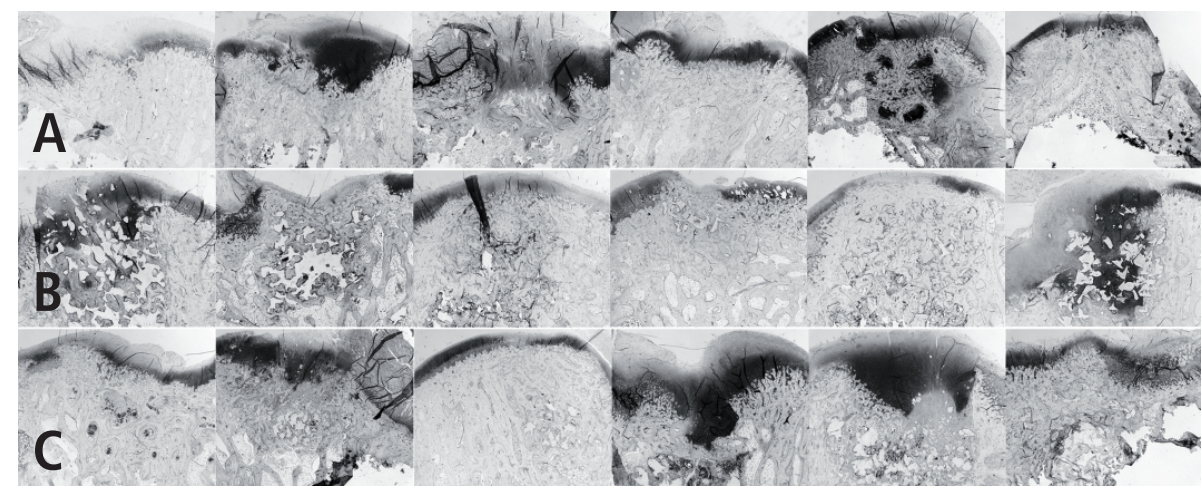

Figure 2. Light micrographs are shown of medial femoral condyles 3 months after creating osteochondral defects. (for full-colour figure see page 199)

A. Untreated osteochondral defects (stain, thionine; original magnification, X25)

B. 55/45 treated osteochondral defects (stain, thionine; original magnification, X25)

C. 70/30 treated osteochondral defects (stain, thionine; original magnification, X25)

Untreated: Figure $2 \mathrm{~A}$ shows the medial femoral condyles 12 weeks after creating OCDs, which were left untreated. OCDs were found to be partly filled with reparative tissue. Disruption of the overlying repair surface by fibrillation or fissures was frequently seen. Staining intensity varied greatly in different areas of the same repair site. While the superficial zone predominantly consisted of fibrous tissue, in the deeper layers of the repair tissue stained cartilage-like tissue was observed (Figure $3 \mathrm{~A}, \mathrm{D})$. However, this tissue was unorganised and contained one or more cysts. In 5 of 6 untreated OCDs a small gap was situated between the repair and the surrounding tissue, indicating poor integration.

55/45: Figure 2 B shows the medial femoral condyles 12 weeks after creating OCDs, which were treated with 55/45 scaffolds. Overall, 55/45 treated OCDs showed repair tissue that predominantly consisted of well-organized bone, and was at level with the native tissue in 4 of 6 samples. Repair tissue completely filled the defect with a thin fibrous tissue layer on top of bone tissue (Figure 3 B, E). In 3 out of 6 samples also cartilage tissue was observed in the superficial layer of the repair tissue. Integration of repair tissue in 55/45 treated OCDs was significantly better as compared to untreated OCDs ( $p=0.027)$ : 5 of $655 / 45$ scaffolds were bonded at both ends with the adjacent native tissue as compared to 1 of 6 in untreated OCDs. Scaffold material was surrounded by few giant cells. Differences in osteochondral repair (O'Driscoll score) between untreated and 55/45 treated OCDs were not significant (Table 3). 


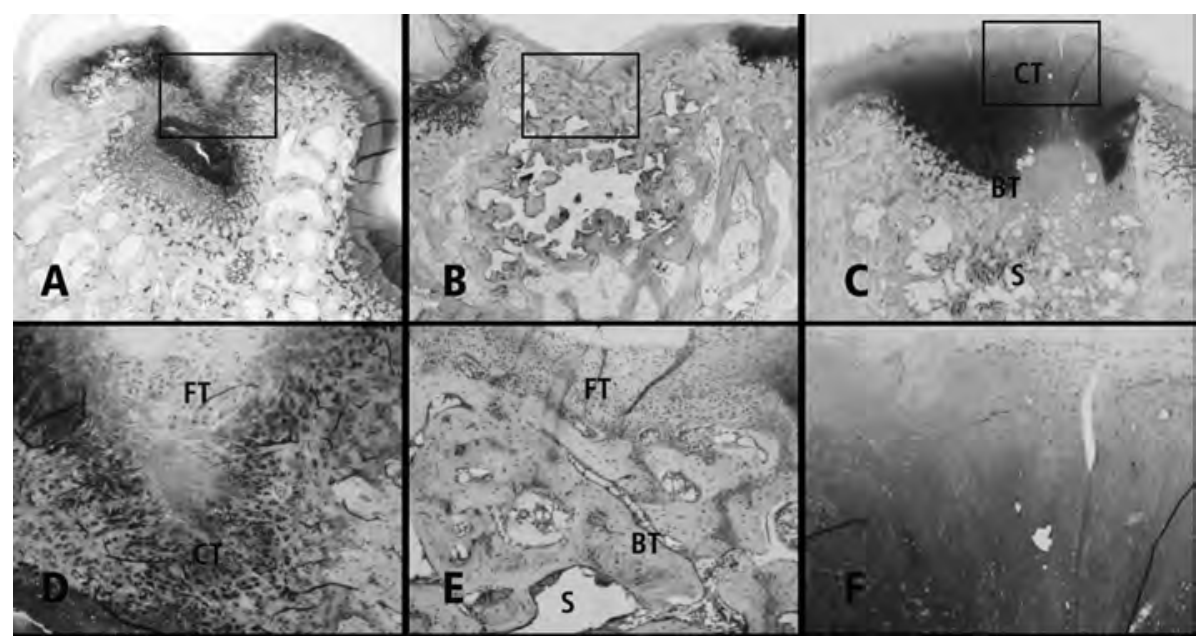

Figure 3. Light micrographs are shown of medial femoral condyles 3 months after creating osteochondral defects. (for full-colour figure see page 200)

A. Untreated osteochondral defect (Stain, thionine; original magnification, X25)

B. 55/45 treated osteochondral defect (Stain, thionine; original magnification, X25)

C. 70/30 treated osteochondral defect (Stain, thionine; original magnification, X25). The defect contains intensively stained cartilage-like tissue (CT), which extended into the subchondral bone. Bone tissue (BT) was situated between cartilage-like tissue and scaffold remnants (S).

D. An enlargement of the box in (A) (stain, thionine; original magnification, X100). The defect is partly filled with reparative tissue consisting of fibrous tissue (FT) in the superficial zone and cartilage-like tissue (CT) containing cysts in the deeper layers.

E. An enlargement of the box in (B) (Stain, thionine; original magnification, $\mathrm{X} 100$ ). The defect contains well-organized bone tissue (BT) with fibrous tissue (FT) on top. Scaffold remnants (S) were observed throughout the osteochondral defect.

F. An enlargement of the box in (C) (Stain, thionine; original magnification, X100).

70/30: Figure $2 \mathrm{C}$ shows the medial femoral condyles 12 weeks after creating OCDs, which were treated with 70/30 scaffolds. The median O'Driscoll score for 70/30 treated OCDs was significantly higher as compared to the untreated and 55/45 treated OCDs $(p=0.024): 17.0$ for the $70 / 30$ group versus 11.5 for the 55/45 and the untreated groups (Table 3). 70/30 treated OCDs were completely filled with reparative tissue and were at level with the surrounding tissue. Repair tissue consisted predominantly of cartilage-like tissue, which extended into the subchondral bone (Figure $3 \mathrm{C}, \mathrm{F}$ ). Matrix staining was in 70/30 treated OCDs significantly better than untreated $(p=$ $0.004)$ and $55 / 45$ treated $(p=0.003)$ OCDs, which indicated proteoglycan 
synthesis in 70/30 scaffolds. A surface alignment of chondrocytes typical of hyaline articular cartilage was never observed. Bone tissue was situated between the cartilage-like tissue and scaffold remnants in the deepest layer of the OCDs (Figure $3 \mathrm{C}$ ), but was situated too low as compared to the surrounding native tissue. Differences between 70/30 and 55/45 scaffolds concerning the level of subchondral bone were statistically indifferent. The repair tissue in 70/30 scaffolds was in 4 of 6 samples fully integrated with the adjacent tissue, which was not significantly different as compared to the integration of 55/45 scaffolds. In contrast to the 55/45 treated OCDs, 3 months after scaffold implantation, an extensive amount of the 70/30 scaffold was already phagocytised, and giant cells were more abundantly present than in the $55 / 45$ counterpart. This underlined the increased degradation of the hydrophilic $70 / 30$ scaffold compared to the more hydrophobic counterpart. In cartilage surrounding the 70/30 treated OCDs chondrocyte clusters were absent (2 of 6 samples); or present but in less than $25 \%$ of the chondrocytes (4 of 6 samples). Besides, the cellularity was normal as compared to untreated and 55/45 treated OCDs. Scores for cellularity and chondrocyte clustering in 70/30 treated OCDs were significantly higher as compared to the $55 / 45$ counterpart $(p=0.005$, and $p=0.014$, respectively). Osteophytes, however, were observed in all samples.

\section{Discussion}

This in vivo rabbit study demonstrated that $70 / 30$ scaffolds performed better in the healing of OCDs than the 55/45 scaffolds, and emphasized the importance of the mechanical and chemical properties of the scaffold.

OCDs were created on the medial femoral condyle of 6-month-old rabbits, hereby reflecting the clinical situation of an OCD in the adolescent patient. ${ }^{3,7,13,189}$ Cell-free scaffolds were implanted, because we were interested in the influence of the scaffolds' mechanical and chemical properties on cartilage repair. For this purpose we compared the 70/30 and 55/45 scaffold. The 70/30 composition showed promising results in vitro concerning chondrocyte attachment, proliferation and differentiation, ${ }^{160}$ whereas the 55/45 scaffold more closely matched the biomechanical properties of articular cartilage. ${ }^{161,162}$

70/30 scaffolds appeared to induce repair tissue that contained cartilage-like tissue on top of subchondral bone. By contrast, most 55/45 scaffolds consisted predominantly of well-developed dense trabecular bone matrix, whereas 
cartilage-formation was observed less frequently. These findings were underlined by the O'Driscoll score for evaluation of the repair tissue in osteochondral defects, which showed significantly better repair tissue in 70/30 as compared to untreated and 55/45 treated OCDs. Interestingly, considering the small deviation in the O'Driscoll score for the 70/30 scaffold further indicates an improvement in the consistency of the healing response in comparison to the 55/45 scaffold. However, repair in OCDs with 55/45 and 70/30 scaffolds showed to have many histologically similar properties. Striking observations were the perfect integration of both scaffolds with the surrounding native tissue and the complete filling of the OCDs. Interestingly, implantation of 70/30 scaffolds resulted in less degenerative changes in the cartilage surrounding the defect as compared to implantation of the 55/45 counterpart, although differences with untreated knees were less evident. It has to be investigated if tissue engineering using PEOT/PBT based scaffolds can prevent cartilage degeneration in the surrounding cartilage.

The observations of the current study could be explained by differences in biomechanical properties. We showed that relatively soft scaffolds performed better in repair of OCDs as compared to scaffolds with relatively high stiffness modules, which is in contrast to the work of Niederauer et al. ${ }^{223}$ who showed that scaffolds with relatively stiff cartilage phases performed better as compared to lower stiffness modules. However, goats were used instead of rabbits, which have different stiffness modules ${ }^{224}$; poly(D,L)lactide-co-glycolide was used as the base material for the scaffolds; and multiphase instead of single-phase scaffolds were used.

Recently, the optimal mechanical properties of a scaffold used in osteochondral defect repair to promote the differentiation of mesenchymal stem cells towards the chondrogenic phenotype were described in a mechanoregulation model. ${ }^{225}$ It is predicted that increasing the stiffness of the scaffold increases the amount of cartilage formation and reduces the amount of fibrous tissue formation in the defect, but this only holds true up to a certain threshold stiffness above which the amount of cartilage formed is reduced. Increasing the Young's modulus of the scaffold to $50 \mathrm{MPa}$ or reducing the modulus to $1 \mathrm{MPa}$ was predicted to have increased amounts of bone and fibrous tissue formation and reduced amounts of cartilage formation within the defect. ${ }^{225}$ Our findings could be explained using this model: i) 55/45 scaffolds were apparently too stiff resulting in increased amounts of fibrous tissue, and less cartilage formation due to progressive osteogenesis; ii) the 70/30 scaffolds supported cartilage formation, consisting of immature cartilage tissue, as predicted in the model, and was observed in this study. Another explanation could be that the low mechanical 
properties and the high degradation rate of the 70/30 scaffold resulted in a decrease in structural integrity, which compromised ingrowth of blood vessels. This hypoxic environment likely enhanced cartilage formation. ${ }^{226}$

Secondly, differences in scaffold chemistry might have influenced the healing response. A more favourable balance between hydrophobic and hydrophilic properties in 70/30 scaffolds might have promoted cartilage formation, whereas decreasing the hydrophilic component, as in 55/45 scaffolds, stimulated bone formation. It was described previously that hydrophobic scaffolds improve bone formation as compared to their hydrophilic counterpart. ${ }^{227}$ Besides, in vitro studies with PEOT/PBT $160,228,229$ showed that a balance of hydrophilic and hydrophobic segments is needed for chondrocyte attachment, while maintaining the chondrogenic phenotype: Chondrocyte attachment and proliferation on films with 55 and $70 \mathrm{wt} \%$ PEOT were similar, but a differentiated phenotype was only observed when films with 70 wt $\%$ PEOT were used. ${ }^{160}$ Besides, polymers with long chain PEO and high PEO content, which is the case in 70/30 scaffolds, are likely to sequester water molecules, providing an environment similar to hyaline cartilage. Moreover, these hydrated conditions may improve nutrient diffusion into scaffold interiors, which supports chondrogenesis throughout the scaffold. ${ }^{220}$

Although the in vivo healing response in the 70/30 scaffold appeared to be better as compared to the 55/45 scaffold, implantation of the $70 / 30$ scaffold was troublesome due to the decrease in stiffness after pre-wetting. The degradation behaviour of PEOT/PBT copolymer in PBS was already shown in in vitro hydrolysis experiments, ${ }^{230}$ and in in vivo studies using gel permeation chromatography following subcutaneous implantation in mini-pigs for 52 weeks. ${ }^{221}$ The hydrophilic 70/30 scaffold showed a twofold increase in the water-uptake in comparison to 55/45, and was almost completely degraded after 52 weeks, whereas the $55 / 45$ scaffold was degraded by only $34 \%$. With the objective of using PEOT/PBT scaffolds in cartilage tissue engineering applications in the human setting, it has to be investigated whether decreasing the hydrophilicity (e.g. 60 wt \% PEOT) will improve scaffold handling without loosing its cartilage inducing properties.

The observed differences in the biological repair response to 55/45 and $70 / 30$ scaffolds indicate the feasibility of using biphasic or bilayered scaffolds for osteochondral repair. A bilayered scaffold comprised of a top layer of soft and hydrophilic 70/30 to stimulate chondrogenesis, and a bottom layer of stiff 
and hydrophobic 55/45 to support subchondral bone formation may have added value for osteochondral tissue engineering. The feasibility of using bilayered technology for this indication, for example, has been shown previously using scaffolds or hydrogels based on oligo(poly(ethylene glycol) fumarate, ${ }^{231} \mathrm{PCL} / \mathrm{PCL}-\mathrm{TCP},{ }^{232}$ hydroxyapatie/chitosan, ${ }^{233}$ and poly-L-lactic acid/hydroxyapatite. ${ }^{234}$ In addition, the biphasic shell-core 3D deposited scaffolds described by Moroni et al. ${ }^{235}$ are also of interest. Fibers comprised of a core of 55/45 and a shell of 70/30 were prepared by exploiting a phase separation phenomenon known as viscous encapsulation and demonstrated favourable properties for in vitro cartilage engineering.

Also, the in vivo behaviour of 55/45 scaffolds seeded with allogenic or autogenic chondrocytes is the subject of further experiments. Besides, long-term investigations need to be undertaken to confirm the longevity of the repair tissue. In summary, filling OCDs with porous scaffolds remains an attractive application in tissue engineering. This study reveals that biomechanical and biochemical properties of a scaffold play an important role by themselves, and can affect the healing response of OCDs. PEOT/PBT based scaffolds with low mechanical properties were superior in cartilage repair tissue formation in OCDs. 



\section{CHAPTER 6}

\section{Human periosteum-derived cells from elderly patients as source for cartilage tissue engineering?}

E.J.P. Jansen, P.J. Emans, N.A. Guldemond, L.W. Van Rhijn, T.J.M. Welting, S.K. Bulstra, R. Kuijer 


\section{Abstract}

The aim of this study was to establish the potential of human periosteumderived cells from elderly patients as cell source for cartilage tissue engineering by optimizing culture conditions of both proliferation and differentiation.

Periosteum was obtained from tibiae of nine patients. Biopsies were prepared for routine histological examination. Periosteum-derived cells were allowed to grow out from the remaining tissue, and were expanded in minimum essential medium containing D-valine (MEM-DV). Fetal bovine serum (FBS) or substitutes, fibroblast growth factor-2 (FGF-2), insulin-like growth factor-1 (IGF1) and non-essential amino acids were added to study proliferation. For differentiation of cells serum-free medium was used supplemented with one or more isoforms of transforming growth factor- $\beta$ (TGFß) and/or IGF-1. Samples were analyzed for expression of collagens type I, II and $X$ by competitive RTPCR, immunohistochemically, and histologically using Alcian Blue staining.

In all samples the cambium layer could hardly be detected. Periosteumderived cells proliferated in serum-containing MEM-DV. Optimal proliferation was found when this medium was supplemented with $100 \mathrm{ng} / \mathrm{mL}$ FGF-2 and nonessential amino acids. Chondrogenesis was detected in $59 \%$ of micromasses that were cultured with TGFß isomers, and in $83 \%$ of the samples cultured in media to which two TGFß isoforms were added.

Periosteum from elderly humans (mean age 66, range 41 - 76) has chondrogenic potential and remains an attractive cell source for cartilage tissue engineering. By expanding cells in MEM-DV, the selection of progenitor cells might be favoured, which would result in a higher cartilage yield for tissue engineering applications. 


\section{INTRODUCTION}

Articular cartilage lesions fail to heal spontaneously and when left untreated evolve to osteoarthritis (OA). ${ }^{236,}{ }^{237}$ Recent research is focused on restoring the articular joint surface with tissue engineered constructs involving scaffolds. ${ }^{238}$ In general, scaffolds in vitro seeded with expanded cells are implanted in the cartilage lesion. ${ }^{238-241}$ Thus far, mature chondrocytes isolated from a cartilage biopsy from a less load-bearing region of the affected joint have been used as the cell source. ${ }^{238,239,242,243}$ Little is known about the fate of the donor site; however, additional injury to the joint surface might contribute to preliminary OA. ${ }^{244}$ Therefore an extra-articular cell source is preferable.

Several groups have proposed the use of mesenchymal stem cells as source for cartilage tissue engineering applications. For instance, human bone marrow and adipose tissue contain populations of mesenchymal stem cells with the capacity to differentiate towards a chondrogenic lineage. ${ }^{245-247}$ When comparing both sources of mesenchymal stem cells, the bone marrow-derived mesenchymal stem cells (BMSCs) appear to differentiate more efficiently into cartilage in comparison to adipose tissue-derived stromal cells (ATSCs). ${ }^{248-250}$ However, the percentage of BMSCs in adult bone marrow is very small, approximately $0.001 \%-0.01 \%$ of isolated cells. ${ }^{251}$ In addition, the heterogeneous nature of bone marrow $^{252,253}$ and adipose tissue ${ }^{254}$ confounds the results of various therapies and often necessitates isolation and purification of the mesenchymal stem cells.

In contrast, periosteum is a relatively pure source of chondrogenic precursor cells, ${ }^{255-257}$ as its histological structure is relatively simple. It contains two distinct layers: a thick outer fibrous layer, adherent to a thin inner cambium layer adjacent to the bone. Mesenchymal stem cells reside in the cambium layer and participate in both osteogenesis and chondrogenesis in both development and fracture healing. ${ }^{255-261}$

In contrast to De Bari et al., ${ }^{262,263}$ neither we nor Nakahara et al. ${ }^{261}$ were able to culture periosteum-derived cells (PDCs) from patients older than 22 years. This was supposed to be due to the diminishing chondrogenic capacity of the periosteum as a consequence of the declining number of stem cells in the cambium layer with age, similar to the situation found in rabbits. ${ }^{264}$ However, most patients who are suffering from a cartilage defect are older, ${ }^{265}$ and therefore we optimized cell culture conditions such that periosteal cells from the periosteum from elderly patients can also be expanded and differentiated.

Since both fibroblasts and mesenchymal stem cells are isolated from 
periosteum, fibroblast overgrowth should be considered. In minimum essential medium containing D-valine (MEM-DV) instead of L-valine, fibroblast growth has been described to be inhibited, because fibroblasts lack the enzyme Damino acid oxidase. ${ }^{266} \mathrm{MEM}-\mathrm{DV}$ has been used in a variety of cell cultures, ${ }^{267-}$ 269 but thus far it was not known whether mesenchymal stem cells could proliferate in the absence of L-valine. We hypothesized that MEM-DV could be used as a selective medium for PDCs.

The aim of the present study was to improve the culture conditions for expanding human PDCs from elderly patients in culture medium formulations based on MEM-DV, including chemically defined ones. In addition, expanded human PDCs were subjected to several combinations of growth factors to improve chondrogenic differentiation.

\section{MATERIALS AND METHODS}

\section{Periosteum harvest and histology}

This study was approved by the medical ethics committee of the University Hospital Maastricht. Informed consent of the patients was obtained prior to inclusion in the study. A 1-2 $\mathrm{cm}^{2}$ sample of periosteum from patients having a knee arthroplasty was isolated from the anteromedial site of the proximal tibia by sharp subperiosteal dissection. Explants were immediately placed in tubes containing phosphate-buffered saline (PBS) supplemented with penicillin (100 $\mathrm{U} / \mathrm{mL})$, streptomycin $(100 \mu \mathrm{g} / \mathrm{mL})$ and amphotericin B $(0.25 \mu \mathrm{g} / \mathrm{mL})$ (Invitrogen, Breda, The Netherlands). In order to examine the presence of the cambium layer, one piece was fixated in $4 \%$ formaldehyde overnight and embedded in paraffin. Routine haematoxylin and eosin (H\&E) staining was used to visualize the cambium layer of the periosteum. The remaining part of the explanted periosteum was used for proliferation and differentiation studies.

\section{Cell isolation}

After rinsing, the periosteum was incubated for three hours at $37^{\circ} \mathrm{C}$ in a shaking waterbath in $5 \mathrm{~mL}$ DMEM-HEPES medium containing a selected batch of type II collagenase (300 U/mL; Invitrogen, Breda, The Netherlands). Collagenase-treated periosteum was transferred into T25 cell culture flasks containing Dulbecco's modified Eagle's medium/Ham's F12 nutrient mix (DMEM/F12; Invitrogen, Breda, The Netherlands), supplemented with $10 \%$ of a selected batch of fetal bovine serum (FBS; Invitrogen) and antibiotics. Cells were allowed to grow out of the tissue. After 7-10 days the periosteum was removed. 
At $80 \%$ confluency, cells were detached from the flask using a trypsin-EDTA solution (Invitrogen) and subcultures were continued in monolayer in MEM-DV (Cell Culture Technologies, Germany) supplemented with 10\% FBS, Lglutamine $(2 \mathrm{mM})$ and antibiotics (culture medium). The medium was refreshed three times/week until sufficient cells were obtained for proliferation or differentiation experiments.

The number of cells considered relevant for these experiments was $2-10 x$ $10^{6}$. This is a number which also comes close to the number of cells required for seeding in a scaffold for tissue engineering for an articular cartilage lesion. Using the above-described procedure, it took approximately 3 months (and seven or eight passages) to reach that number. Proliferation assays were therefore performed to evaluate the use of growth factors to speed up the process. For these experiments we used approximately $1 \times 10^{6}$ cells from two different samples.

\section{Proliferation assay}

PDCs from 2 patients (females aged 67 and 71 years; passage 7) were seeded in culture medium in four 96 -well plates (1000 cells/well) and were allowed to attach for 1 day. Subsequently, 24 different culture medium recipes were tested in quadruplicate (Table 1) in four parallel 96-well plates. At days 1, 6, 11 and 14, one 96-well plate with cell cultures was terminated and the DNA content of the wells was assessed using the CyQUANT ${ }^{\circledR}$ DNA Assay kit according to the instructions of the manufacturer (Molecular Probes, Leiden, The Netherlands). In brief, $200 \mu \mathrm{L}$ CyQUANT ${ }^{\circledR}$ GR dye/cell lysis buffer was added to each sample. An aliquot of each sample was incubated for 5 minutes at room temperature protected from light exposure. The sample fluorescence was measured at 480 $\mathrm{nm}$ excitation and $520 \mathrm{~nm}$ emission wavelengths. Fluorescence measurements were compared with the values obtained from a standard DNA curve. Growth factors such as fibroblast growth factor-2 (FGF-2) and insulin-like growth factor1 (IGF-1) (both from R\&D, Uithoorn, The Netherlands) were applied in different concentrations as well as in combinations to serum-free or serum-containing MEM-DV. Also, the addition of non-essential amino acids (neAA) (100X diluted; Invitrogen) was tested. In serum-free medium formulations, serum substitutes were applied: $1 \%$ insulin-transferrin-selenium (ITS; Invitrogen) and Ultroser ${ }^{\circledR} G$ (Invitrogen). ${ }^{270-272}$ Bovine serum albumin (BSA; Sigma-Aldrich Chemie B.V., Zwijndrecht, The Netherlands) was added as a protein source.

\section{In vitro chondrogenic differentiation assay}

PDCs from seven patients (mean age 65, range 41-76; SD=13) were harvested, in monolayer expanded in culture medium (as described above) without use of growth 
Table 1. Culture medium additives tested in the proliferation assay. Standard culture medium was minimum essential medium containing D-valine supplemented with fetal bovine serum, penicillin, streptomycin, amphotericin and L-glutamine. Also culture medium without fetal bovine serum was tested.

\begin{tabular}{|c|c|c|c|c|c|c|c|c|c|}
\hline \multirow[t]{3}{*}{ Condition } & \multirow{3}{*}{$\begin{array}{c}\text { FBS } \\
(10 \%)\end{array}$} & \multicolumn{2}{|c|}{ FGF-2 } & \multicolumn{2}{|c|}{ IGF-1 } & \multirow{3}{*}{$\begin{array}{l}\text { neAA } \\
(1 \%)\end{array}$} & \multirow{3}{*}{$\begin{array}{l}\text { ITS } \\
(1 \%)\end{array}$} & \multirow{3}{*}{$\begin{array}{c}\text { Ultroser }{ }^{\circledR} \mathrm{G} \\
(1 \%)\end{array}$} & \multirow{3}{*}{$\begin{array}{c}\text { fold } \\
\text { increase } \\
14 \text { days }\end{array}$} \\
\hline & & 10 & 100 & 10 & 300 & & & & \\
\hline & & \multicolumn{2}{|c|}{ (ng/mL) } & \multicolumn{2}{|c|}{ (ng/mL) } & & & & \\
\hline 1 & + & - & - & - & - & - & - & - & 21 \\
\hline 2 & + & + & - & - & - & - & - & - & 40 \\
\hline 3 & + & - & + & - & - & - & - & - & 32 \\
\hline 4 & + & + & - & - & - & + & - & - & 26 \\
\hline 5 & + & - & + & - & - & + & - & - & 49 \\
\hline 6 & + & + & - & + & - & - & - & - & 25 \\
\hline 7 & + & - & + & + & - & - & - & - & 37 \\
\hline 8 & + & + & - & - & + & - & - & - & 35 \\
\hline 9 & + & - & + & - & + & - & - & - & 37 \\
\hline 10 & + & + & - & + & - & + & - & - & 44 \\
\hline 11 & + & - & + & + & - & + & - & - & 44 \\
\hline 12 & + & + & - & - & + & + & - & - & 44 \\
\hline 13 & + & - & + & - & + & + & - & - & 40 \\
\hline 14 & - & + & - & - & - & - & + & - & 1 \\
\hline 15 & - & - & + & - & - & - & + & - & 1 \\
\hline 16 & - & + & - & - & + & - & + & - & 1 \\
\hline 17 & - & - & + & - & + & - & + & - & 1 \\
\hline 18 & - & + & - & + & - & + & + & - & 1 \\
\hline 19 & - & - & + & + & - & + & + & - & 1 \\
\hline 20 & - & - & - & - & - & - & - & + & 1 \\
\hline 21 & - & + & - & - & - & + & - & + & 1 \\
\hline 22 & - & - & + & - & - & + & - & + & 1 \\
\hline 23 & - & + & - & + & - & - & - & + & 1 \\
\hline 24 & - & - & + & + & - & - & - & + & 1 \\
\hline
\end{tabular}

factors, and subsequently cultured in micromasses, as described elsewhere. ${ }^{262,263}$ Approximately $4 \times 10^{5}$ cells of passage $6-7$ were used to prepare micromass cultures by centrifugation at $200 \times g$ for 4 minutes. The resulting micromasses were 
cultured in DMEM, supplemented with $1 \%$ ITS and $0.2 \mathrm{mM}$ ascorbic acid-2phosphate (AA-2-P; differentiation medium; Sigma-Aldrich) IGF-1 (300 ng/mL) and transforming growth factor- $\beta$ (TGFß) isomers $(10 \mathrm{ng} / \mathrm{mL} ; \mathrm{R} \& D)$ were added to the culture medium to study their possible stimulatory effect on periosteal chondrogenesis. Various subtypes of TGFß were used $\left(\mathrm{TGF}_{1}, \mathrm{TGF}_{2}\right.$, and TGF $\left.\beta_{3}\right)$. Controls were micromasses cultured in differentiation medium without growth factors. Media, including growth factors, were refreshed twice weekly. Cultures were maintained for 3 weeks and a minimum of six micromasses was collected for isolation of total RNA. Besides, $5 \mu \mathrm{m}$ thick cryosections were prepared and stained with Alcian blue to detect proteoglycans in micromasses, which was supposed to be indicative for chondrogenic differentiation.

For immunohistochemical analysis of chondrogenic differentiation, the method described by Mandl et al. was used. ${ }^{273}$ Briefly, PDCs cultured in alginate beads 273,274 were exposed to the conditions that were tested. The alginate was dissolved in a solution of $55 \mathrm{mM}$ sodium citrate, $150 \mathrm{mM}$ of sodium chloride and $20 \mathrm{mM}$ EDTA. The cells were collected by centrifugation, rinsed in PBS and centrifuged in a cytospin centrifuge. Cytospins were fixated in acetone for 15 minutes at $4^{\circ} \mathrm{C}$, washed $3 \times 5$ minutes in PBS and preincubated in PBS containing $20 \%$ FBS for 10 minutes. The cytospin preparations were stained with antibodies against CD90 (mouse monoclonal anti-Thy-1, MAB1294; Brunschwig Chemie, Amsterdam, The Netherlands), which we used as a marker for non-chondrogenic cells (in periosteum primarily fibroblasts), collagen type I (Developmental Studies Hybridoma bank (DSHB), lowa University, USA), collagen type II (II-II6B3, DSHB; lowa University, USA) and collagen type X (X53, Quartett diagnostics, Berlin, Germany). The secondary antibody was rabbit anti-mouse immunoglobulin $G$ conjugated with horseradish peroxidase (HRP) at 1: 200 (Dako, Glostrup, Denmark). The secondary antibody was detected using 3.3-diaminobenzidine (DAB) (Merck, Darmstadt, Germany).

\section{RNA isolation and competitive RT-PCR}

Total RNA was isolated using TriZol ${ }^{\mathrm{TM}}$ (Invitrogen) according to the manufacturer's instructions. The competitive RT-PCR method has been described previously. ${ }^{275}$ Genomic DNA was removed from the samples by RNase-free DNase I digestion (Roche, Woerden, The Netherlands). Absence of genomic DNA was controlled with a PCR using RNA as a template and the primers for glyceraldehyde 3-phosphate dehydrogenase (GAPdH). To $200 \mathrm{ng}$ total RNA different amounts of standard RNA were added. The samples were reverse-transcribed for 1 hour at $37^{\circ} \mathrm{C}$, using $100 \mathrm{U}$ Moloney murine leukaemia 
virus (M-MLV) reverse transcriptase (Promega Corporation, Leiden, The Netherlands) in $50 \mathrm{mM}$ Tris- $\mathrm{HCl}, \mathrm{pH} 8.3,75 \mathrm{mM} \mathrm{KCl}, 3 \mathrm{mM} \mathrm{MgCl}$ and $10 \mathrm{U}$ RNAsin and random hexamer primers (Promega, WI, USA). CDNA $(2 \mu \mathrm{L})$ from each resulting sample was amplified in $10 \mu \mathrm{L}$ PCR reaction mixture with primers for either type I, II or X collagen. The primer set for collagen type II was obtained from the literature, ${ }^{276,277}$ primer sets for GAPdH, collagen type I and type $X$ were selected from the sequences of the cDNA obtained from Genbank (Table 2). PCR products were subjected to agarose gel electrophoresis and DNA bands were visualized. Images of the gels were digitized, and bands were quantified using Geldoc 1000 system using Multi-Analist software (Bio-Rad, CA, USA).

Table 2. Primer sequences for GAPdH, and collagens type I, II and X

\begin{tabular}{|c|c|c|c|c|}
\hline cDNA & \multicolumn{2}{|c|}{ Size (bp) } & Primer sequence & Genbank Accession No. \\
\hline \multirow[t]{2}{*}{ GAPdH } & 235 & a & 5'-CGGCCATCACGCCACAGTTT-3' & BC083511 \\
\hline & & as & 5'-TGCTGATGCCCCCATGTTCG-3' & \\
\hline \multirow[t]{2}{*}{ Collagen type I } & 552 & a & 5'-GGCCACGCTGTTCTTGCAGT-3' & 274615 \\
\hline & & as & 5'-CCAGCGCTGGTTTCGACTTC-3' & \\
\hline \multirow[t]{2}{*}{ Collagen type II } & 492 & a & 5'-GAAAAGATGGTCCCAAAGGTGC-3' & \\
\hline & & as & 5'-TGTCTCCTTGCTTGCCAGTTGG-3' & \\
\hline \multirow[t]{2}{*}{ Collagen type X } & 329 & a & 5'-ACAGGAATGCCTGTGTCTGCTTTT-3' & AY598937 \\
\hline & & as & 5'-TTGGGAAGCTGGAGCCACACCTGGTC-3' & \\
\hline
\end{tabular}

\section{Statistical analysis}

Differences between cell cultures concerning the proliferation rate over the course of 14 days were analyzed using repeated measurements analysis of variance (ANOVA). Differentiation data were analyzed according to ANOVA procedures with Bonferroni post hoc comparison. Differences between micromass cultures concerning percentages of positive type II collagen mRNA expressions were analyzed according the Pearson $\chi^{2}$ procedure with Yates correction. Values of $p<0.05$ were considered to indicate statistically significant differences. 


\section{RESULTS}

In all periosteal explants the cambium layer was hard to detect using routine histology. None or only a single layer next to a thick fibrous layer was observed (Figure 1). Outgrowth of cells from collagenase-treated periosteum was evident in all samples.

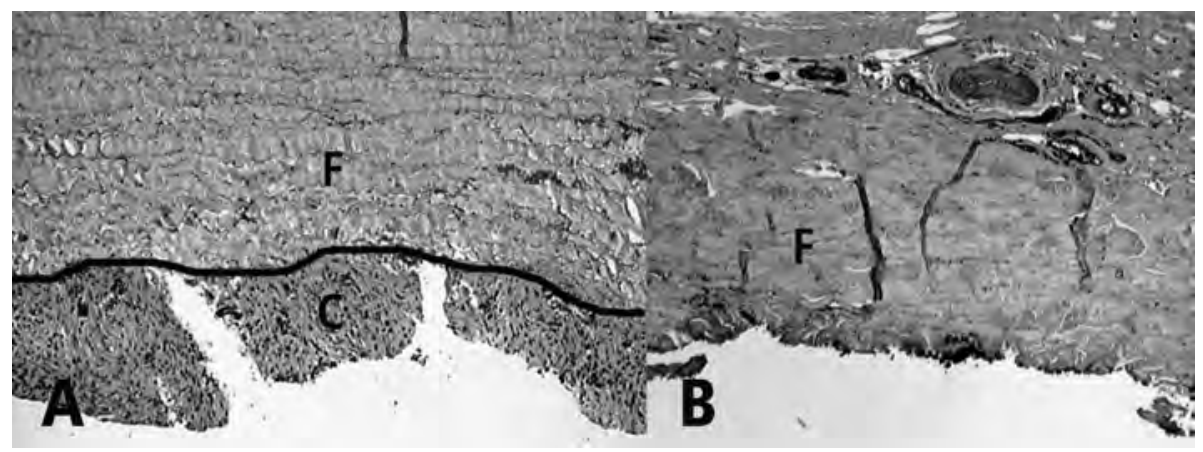

Figure 1. Micrographs of periosteum explants harvested from A) a young patient (age 5 years) undergoing an epiphysiodesis using a staple technique, approximately $2 \mathrm{~cm}$ caudally from the proximal tibial growth plate; and B) the proximal tibia from an elderly patient (age 62 years). Notice the clearly distinguishable cambium layer (c) in the periosteum derived from the young patient and the hardly detectable cambium layer in the periosteum from the elderly patient. The overlying fibrous layer $(\mathrm{F})$ contains fibroblasts. The line in A) separates the fibrous (F) and cambium (C) layer. Magnification: 50X

\section{Proliferation assay}

(for full-colour figure see page 201)

PDCs in monolayer culture did proliferate in DMEM/F12 culture medium supplemented with $10 \%$ FBS. Subsequent chondrogenic differentiation of the culture-expanded PDCs failed using the standard differentiation conditions. An overgrowth of fibroblasts was assumed and, in order to reduce fibroblast growth PDCs were cultured in MEM-DV supplemented with $10 \%$ FBS. In this medium PDCs grew and the expanded cells did show chondrogenic differentiation. Since the expansion rate was low, several growth factors and combinations thereof were tested to increase the expansion rate (Table 1). FBS supplementation appeared to be essential to obtain any expansion of the PDCs. In serum-free culture media supplemented with either ITS or Ultroser ${ }^{\circledR} \mathrm{G}$ as serum substitute PDCs did not proliferate independent of the addition of growth factors like FGF2 or IGF-1 or combinations thereof.

In comparison to serum-free medium, 10\% FBS supplementation increased the cell proliferation 21-fold in 14 days (Table 1, Figure 2). Additional 
supplementation with $10 \mathrm{ng} / \mathrm{mL}$ or $100 \mathrm{ng} / \mathrm{mL}$ FGF-2 resulted in an at least 32fold increase of cell expansion compared to the serum-free condition at 14 days $(p<0.001$; Table 1, Figure 2 B). The combination of FGF-2 and IGF-1 showed an additional enhancement of proliferation compared to FGF-2 alone (Figure 2 B). Addition of neAA to FGF-2-containing media resulted in a small but significant increase in proliferation $(p=0.01)$. The highest cell number was obtained in serum-containing MEM-DV supplemented with $100 \mathrm{ng} / \mathrm{mL}$ FGF-2 and neAA (Table 1, Figure $2 \mathrm{~A}$ ).

\section{In vitro chondrogenesis assay}

Chondrogenic differentiation experiments started after an average of seven passages (range 5-11; SD 2, average, 175 days; SD 37). Ten different combinations of growth factor-containing media were tested (Table 3).

Table 3. Chondrogenic differentiation of micromass cultures of PDCs.

\begin{tabular}{|c|c|c|c|c|c|c|c|}
\hline \multirow[t]{2}{*}{ Growth factor } & \multirow{2}{*}{$\begin{array}{c}\text { samples } \\
n_{\text {test }}\end{array}$} & \multicolumn{2}{|c|}{ collagen type I } & \multicolumn{2}{|c|}{ collagen type II } & \multicolumn{2}{|c|}{ collagen type $X$} \\
\hline & & $n$ & expr & $n$ & expr & $n$ & expr \\
\hline none & 6 & 6 & ++ & 0 & & 5 & + \\
\hline $\mathrm{TGFB}_{1}$ & 2 & 2 & +++ & 1 & ++ & 2 & ++ \\
\hline $\mathrm{TGFB}_{2}$ & 4 & 4 & ++ & 1 & + & 3 & + \\
\hline $\mathrm{TGFB}_{3}$ & 3 & 3 & ++ & 2 & ++ & 2 & ++ \\
\hline $\mathrm{TGFB}_{1}+\mathrm{TGFB}_{2}$ & 2 & 2 & ++ & 2 & + & 2 & + \\
\hline $\mathrm{TGFB}_{1}+\mathrm{TGFB}_{3}$ & 2 & 2 & +++ & 1 & + & 2 & +++ \\
\hline $\mathrm{TGFB}_{2}+\mathrm{TGFB}_{3}$ & 2 & 2 & +++ & 2 & + & 2 & +++ \\
\hline IGF-1 & 2 & 2 & ++ & 0 & & 1 & + \\
\hline$I_{G F}-1+T G F \beta_{1}$ & 4 & 4 & +++ & 3 & + & 4 & ++ \\
\hline IGF-1+TGFB 2 & 3 & 3 & ++ & 1 & + & 2 & ++ \\
\hline $\mathrm{IGF}-1+\mathrm{TGFB}_{3}$ & 5 & 5 & ++ & 3 & + & 5 & ++ \\
\hline \multicolumn{8}{|c|}{ The absolute expression of the markers collagen types I, II and X by RT- PCR is given. } \\
\hline \multirow{3}{*}{\multicolumn{8}{|c|}{$\begin{array}{l}\text { PDCs were differentiated in micromass cultures in DMEM supplemented with } 1 \% \text { ITS and } 0.2 \mathrm{mM} \text { ascorbic acid-2-phospha } \\
\text { Different isoforms of TGFB were tested alone and in combination with IGF-1. Final concentrations of } 10 \mathrm{ng} / \mathrm{mL} \text { were } \\
\text { used for TGFB, TGFB } 2 \text { and TGFB } 3300 \mathrm{ng} / \mathrm{mL} \text { for IGF-1. }\end{array}$}} \\
\hline & & & & & & & \\
\hline & & & & & & & \\
\hline \multicolumn{8}{|c|}{ Mean mRNA quantities per 200 ng total RNA are designated: +< $500 \mathrm{fg} ;++$ between $500 \mathrm{fg}$ and 50 pg; +++ > 50 pg. } \\
\hline \multicolumn{8}{|c|}{$n_{\text {test }}-$ number of samples tested; $n$ - number of samples which show positive expression. } \\
\hline
\end{tabular}


A

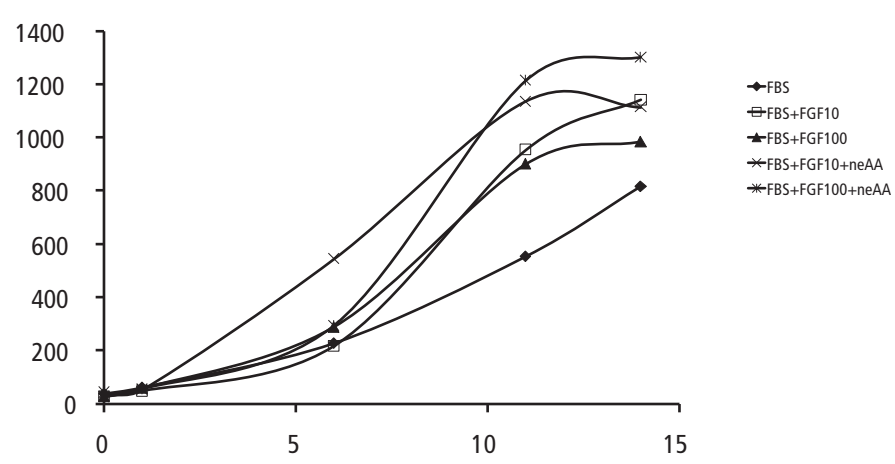

B
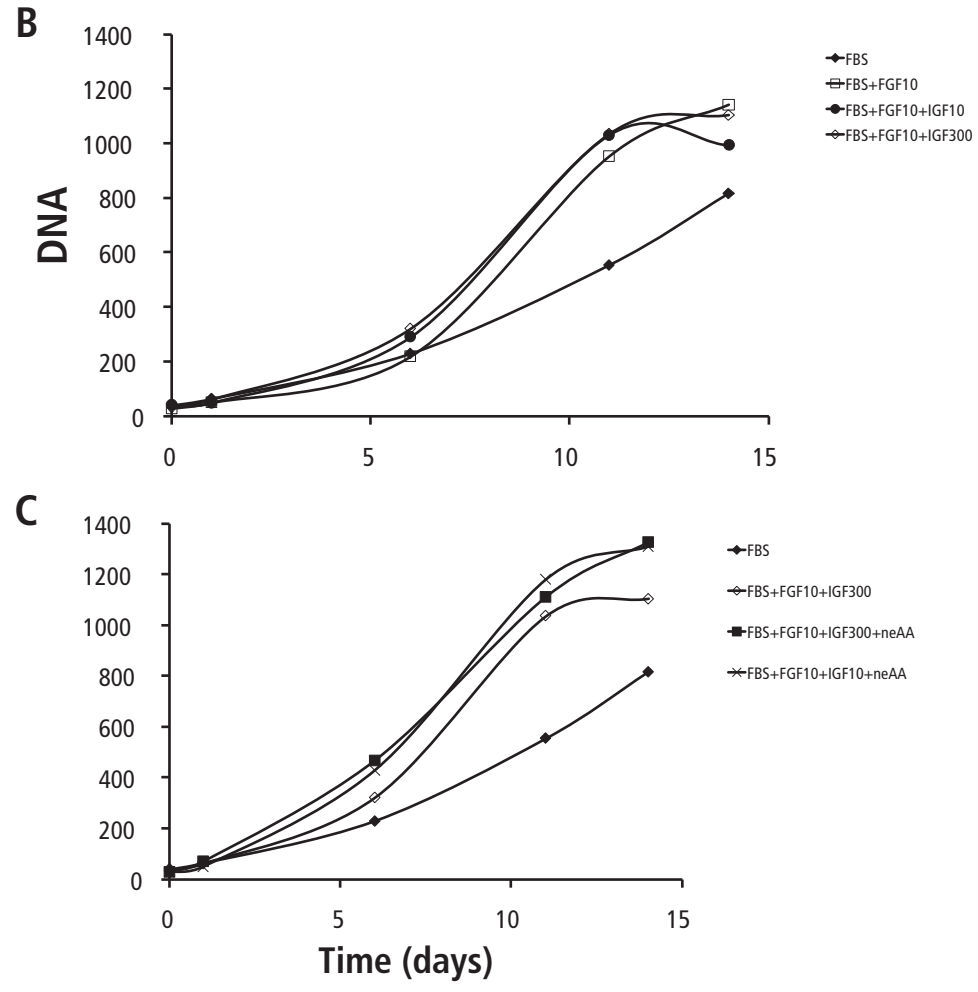

Figure 2. Growth curves of elderly human periosteum-derived cells expanded in minimum essential medium containing D-valine (MEM-DV). (A) Effects of addition of two different concentrations of FGF-2 to MEM-DV containing fetal bovine serum (FBS) and subsequent addition of non-essential amino acids (neAA); (B) effects of addition of different concentrations of IGF-1 to MEM-DV containing FBS and $10 \mathrm{ng} / \mathrm{mL}$ FGF-2; (C) effects of addition of different combinations of FGF-2 and IGF-1 to MEM-DV containing FBS. 
The addition of TGFß isomers resulted in a significantly $(p<0.01)$ higher percentage of samples that expressed type II collagen mRNA (59\%) compared to controls $(0 \%)$ and cultures to which only IGF- 1 was added $(0 \%)$. The highest percentage of samples that expressed type II collagen mRNA was observed in cultures to which two different TGFß isomers were added (83\%), compared to $52 \%$ of the cultures to which one TGFß isomer was added. The amount of collagen type II mRNA found was variable but highest in micromasses with $\mathrm{TGF}_{1}$ or TGFß $\beta_{3}$ (500 fg - $50 \mathrm{pg}$ of $200 \mathrm{ng}$ total RNA). There was no significant difference between the TGF $B$ isomers in stimulating chondrogenic differentiation.

Expression of type I collagen mRNA was detected in all samples; type $\mathrm{X}$ collagen mRNA in $83 \%$ of control and in $86 \%$ of growth factor-containing samples (500 fg - $50 \mathrm{pg}$ of $200 \mathrm{ng}$ total RNA). The presence of collagen type $\mathrm{X}$ mRNA indicated that the chondrogenic differentiation will most likely result in hypertrophic chondrocytes, although we were not able to detect the collagen type $\mathrm{X}$ protein (data not shown).

These results were underlined by histologic analyses of micromass cryosections. Cartilage-like tissue was demonstrated in all growth factor-treated micromasses. Occasionally, in micromass cultures stimulated with TGFß $\beta_{3}$ a zone of calcification was observed (Figure 3).
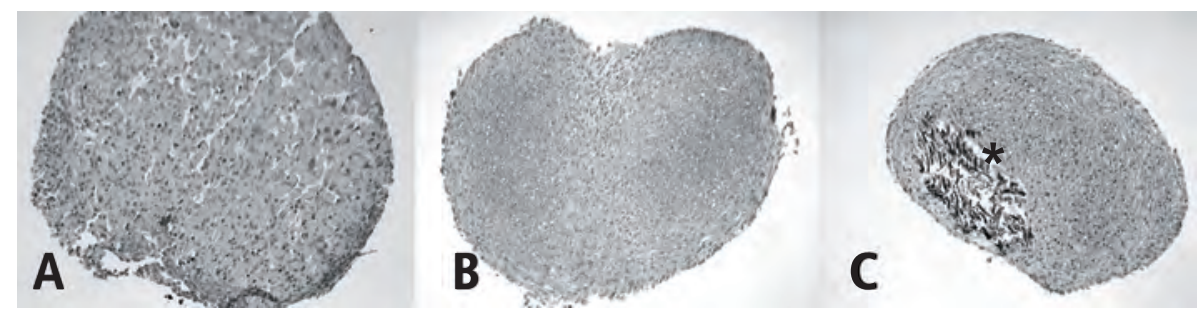

Figure 3. Micrographs of cryosections of micromasses after staining with Alcian blue (donor age was 62 years). Periosteum-derived cells were differentiated in medium A) without growth factors or medium supplemented with B) TGFß 1 (10 ng/mL) or C) TGFß $\beta_{3}(10 \mathrm{ng} / \mathrm{mL})$. Notice the zone of calcification $(*)$ in the micromass that was differentiated with $\mathrm{TGF}_{3}$.

Magnification: X50 (for full-colour figure see page 201)

Immunohistochemical data on cytospin preparations of PDCs cultured in alginate corroborated with the data from both RT-PCR and histology. Collagen type II was expressed at the protein level by the majority of the cells. Collagen type I protein synthesis was less evident (Figure 4). The fibroblast marker Thy-1 was present in fibroblasts, but far less in PDCs after chondrogenic differentiation in alginate beads. 

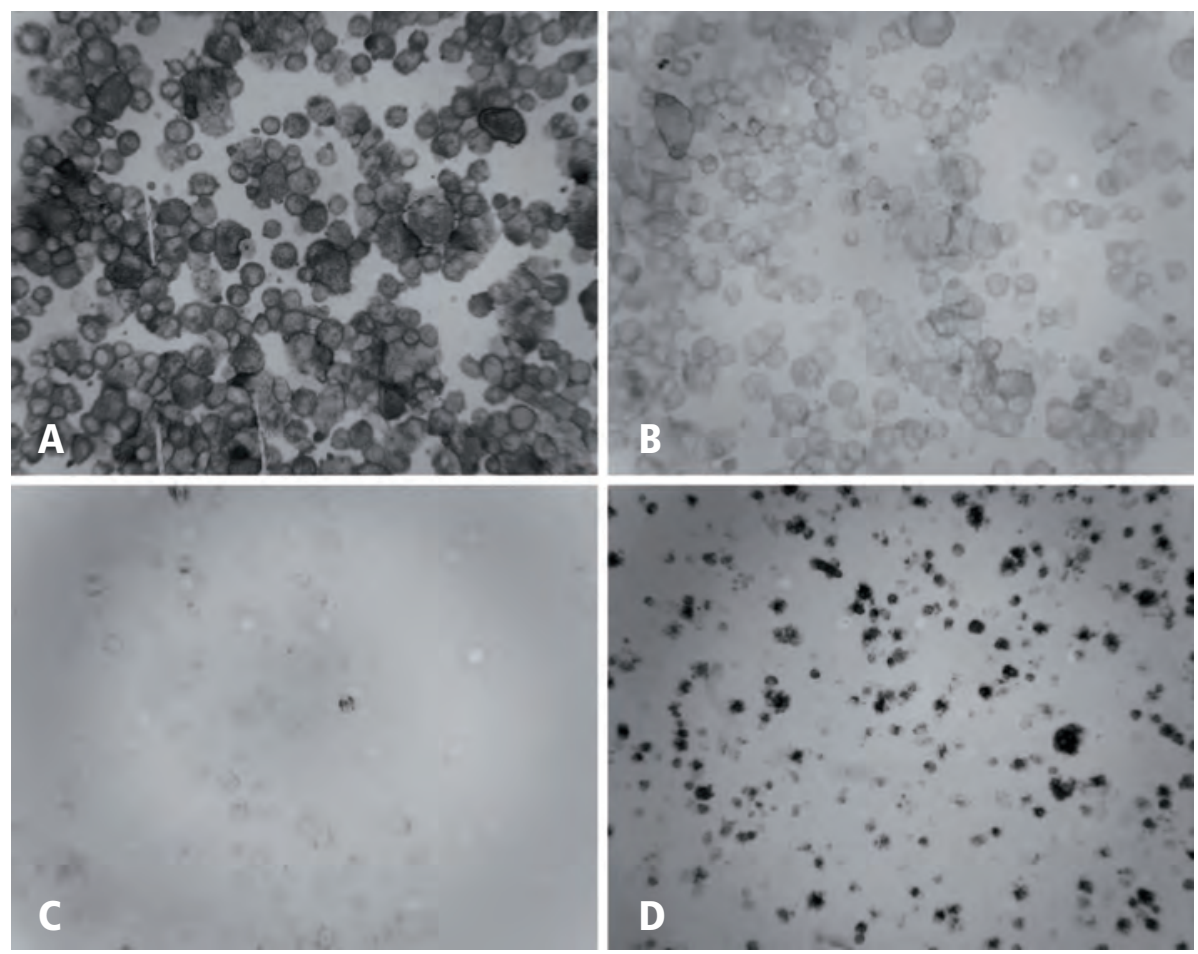

Figure 4. Cytospins of (A) human embryonic lung fibroblasts stained with an antibody for Thy-1 (positive control); (B) periosteum-derived cells from elderly patient stained with anti-Thy-1; (C) periosteum-derived cells from elderly patient stained with anti-collagen type I (M38, DSHB); (D) periosteum-derived cells from elderly patient stained with anti-collagen type II (II-II6B3, DSHB). Secondary antibodies were conjugated with horseradish peroxidase. Diaminobenzidine was used to develop the colour (brown is positive signal). (for full-colour figure see page 202)

\section{Discussion}

This study was performed to develop a procedure for the isolation and subsequent culture of PDCs as an alternative cell source for cartilage tissue engineering. Procedures described by others were either cumbersome to perform, ${ }^{256}$ using various filters and subfractions of cell isolates, or in our hands did not result in cells that were able to differentiate into the chondrogenic lineage. ${ }^{262}$ We assume that the cells from the cambium layer of the periosteum are the mesenchymal stem cells, capable of differentiation into mesenchymal lineages. The fibrous layer of periosteum contains fibroblasts, cells that readily 
grow in cell culture and tend to overgrow other cell types. This was probably the case in our first attempts to culture expand PDC cultures according to De Bari et al.

Despite extensive searches for discriminating cell surface markers to differentiate between fibroblasts and periosteal mesenchymal stem cells, these have not yet been discovered. Recent studies suggests that a combination of markers can be used to obtain a significantly enriched population of stem cells from periosteum using a fluorescence-activated cell sorter (FACS). ${ }^{278,279}$ Such a procedure in combination with a selective cell culture medium may significantly increase the potential of periosteum as a source of mesenchymal stromal cells.

In order to isolate and purify epithelial cells, Gilbert and Migeon developed a culture medium which inhibits the growth of fibroblasts. ${ }^{266}$ In this medium Lvaline is replaced by D-valine. Since fibroblasts lack the enzyme D-amino acid oxidase, these cells will remain present in cell cultures, but their growth will be arrested in the $\mathrm{G}_{0}$ phase of the cell cycle. The presence of fibroblasts was not considered to be a problem, since fibroblasts have been shown to have a positive effect on the chondrogenic capacity of mesenchymal stromal cells. ${ }^{280}$ Our first attempts to culture differentiating PDCs using standard culture media, such as based on DMEM/Ham's F12 or DMEM, failed. The cells we cultured proved to be highly positive for CD90 (Thy-1) and did not express any of the chondrocyte markers. Culture of PDCs in MEM-DV supplemented with 10\% FBS resulted in cells capable of differentiating into the chondrogenic lineage. Apparently this medium suppresses the growth of fibroblasts at the same time allowing proliferation of PDCs.

Several growth factors and nutrients were added in order to accelerate cell proliferation in MEM-DV. The addition of FGF-2, IGF-1 and neAA to serumcontaining medium increased the proliferation rate of PDCs. FGF-2 had a positive effect on PDCs that proliferated in monolayer, probably by stimulating their mitotic activity. ${ }^{25,} 281$ Similar results were found when BMSCs or ATSCs were cultured in the presence of FGF-2. ${ }^{282-284}$

IGF-1 has been described not to enhance the proliferation of human BMSCs. ${ }^{285}$ In our study, IGF-1 did enhance the growth of human PDCs in the presence of FGF2. Also non-essential amino acids apparently were a rate-limiting factor, since addition resulted in a small but significant stimulation of PDC growth. The use of FBS proved to be essential for the proliferation of PDCs in our experiments. PDCs did not grow in culture media in which FBS was replaced by serum substitutes such as ITS 
or Ultroser ${ }^{\circledR} \mathrm{G}$. A chemically defined culture medium has been described for rat bone marrow stromal cells, ${ }^{280}$ but never for human BMSCs. Recent studies show that FBS can be replaced by human platelet lysate, ${ }^{286,287}$ which significantly increased cell growth as well as improved maintenance of the stem cell phenotype. ${ }^{286}$ It is not yet clear whether human platelet lysate will also be useful for the culture of human PDCs, enhancing proliferation and reducing differentiation.

Results from studies thus far undertaken to compare human mesenchymal stem cells (MSCs) from different sources are difficult to interpret, since in most studies one culture medium is used for expansion of the different MSCs. ${ }^{248-250}$ From this study it is apparent that human PDCs require a different culture medium than either human BMSCs or human ATSCs.

Differentiation towards a chondrocyte phenotype was achieved in representative high-density cell culture systems with addition of growth factors that are normally present in articular cartilage. Regardless of the TGFß isomer used, type II collagen mRNA was expressed in 59\% of the samples to which exogenous TGF $\beta$ was added.

The stimulatory effect of exogenous administered $T G F \beta_{1}$ has been described previously. ${ }^{256-260,262}$ TGF $_{2}$ also has a stimulatory effect on chondrogenesis from human $\mathrm{BMSCs}^{288}$ but was never used in periosteal differentiation studies. TGF $\beta_{3}$ stimulated chondrogenesis in human BMSCs, ${ }^{289}$, 290 as well as in human PDCs. ${ }^{291}$ Cells cultured with IGF-1 alone never expressed type II collagen, which is in contrast to periosteal explant culture studies, in which IGF-1 increased chondrogenesis when administered throughout the culture period. ${ }^{292,}{ }^{293} \mathrm{~A}$ possible explanation could be that IGF-1 only has a pivotal role in periosteal chondrogenesis when used in combination with TGFß ${ }^{292}$ More likely is that isolated PDCs behave and respond differently than cells within their matrix, in an explant.

Type X collagen mRNA was abundantly present in PDCs after chondrogenic differentiation, indicating that PDCs will most likely end up as hypertrophic chondrocytes. However, the collagen type $X$ protein was not detected in the samples we examined with the X53 antibody; its expression may be regulated at a translational level. We observed calcification in a micromass culture of PDCs cultured in the presence of TGF $\beta_{3}$. This is indicative of terminal differentiation of the chondrocytes in this culture. Chondrogenic differentiated human BMSCs and ATSCs also express collagen type $\mathrm{X}$ and show features of hypertrophic chondrocytes. ${ }^{250}$ Hypertrophic differentiation of mesenchymal stromal cells in general is an issue of concern. Several attempts have been done to control this process. Addition of a synthetic inhibitor of the 
retinoic acid receptor to human BMSCs in culture induced chondrogenesis and inhibited hypertrophic differentiation. ${ }^{294}$ In a clinical study on autologous perichondrium transplantation, calcification of the transplanted tissue was observed. Using the protocol to inhibit ectopic calcification after total hip arthroplasty, administration of indomethacin for 14 days starting the day before surgery, such calcifications could be considerably inhibited. ${ }^{130,153}$ This is indicative for a role of cyclooxygenase and prostaglandins in the process of hypertrophic differentiation.

Whether bone morphogenetic protein-6 (BMP-6), which enhances chondrogenesis in human ATSCs, ${ }^{295,} 296$ has a similar effect on human PDCs requires additional investigation. In these studies the role of BMP-6 on hypertrophic differentiation was not investigated.

PDCs from elderly people appear to be a potential cell source for cartilage tissue engineering applications, which corroborates with previous literature reports. A number of possible improvements to the currently described protocol can still be made. First, the surgical technique of harvesting periosteum is reported to be critical. ${ }^{297}$ The cambium layer of the elderly patient is, in contrast to the young patient, very thin. ${ }^{264}$ It is known that the presence of this layer is essential for periosteal chondrogenesis in vitro. ${ }^{255}, 257-259$ We harvested periosteal explants by sharp subperiosteal dissection instead of using a periosteal elevator ("gold standard"). Since the mesenchymal stem cells adhere only lightly to the periosteum, they may be left at the bone surface. Secondly, the thin cambium cell layer in the elderly patient makes a good control of mesenchymal stem cells after harvesting virtually impossible. A validated method to warrant the quality of the periosteum sample immediately after harvesting appears to be a prerequisite, considering the ultimate number of collagen type II - producing PDC samples. Cambium layer cells within periosteum stain positive for endogenous alkaline phosphatase activity. ${ }^{298}$ Whether this simple staining procedure can be used to assess the quality of the periosteum sample needs to be validated.

In summary, periosteum from elderly human beings has chondrogenic potential and remains an attractive cell source for cartilage tissue engineering. Among the minimal morbidity by which it can be obtained, it is a relatively pure source of mesenchymal stem cells that can differentiate into a chondrogenic lineage. By expanding cells in MEM-DV, the selection of mesenchymal stem cells might be favoured, which will result in a higher cartilage yield for tissue engineering applications. 
Human PDCs from elderly patients as a source for cartilage tissue engineering? 


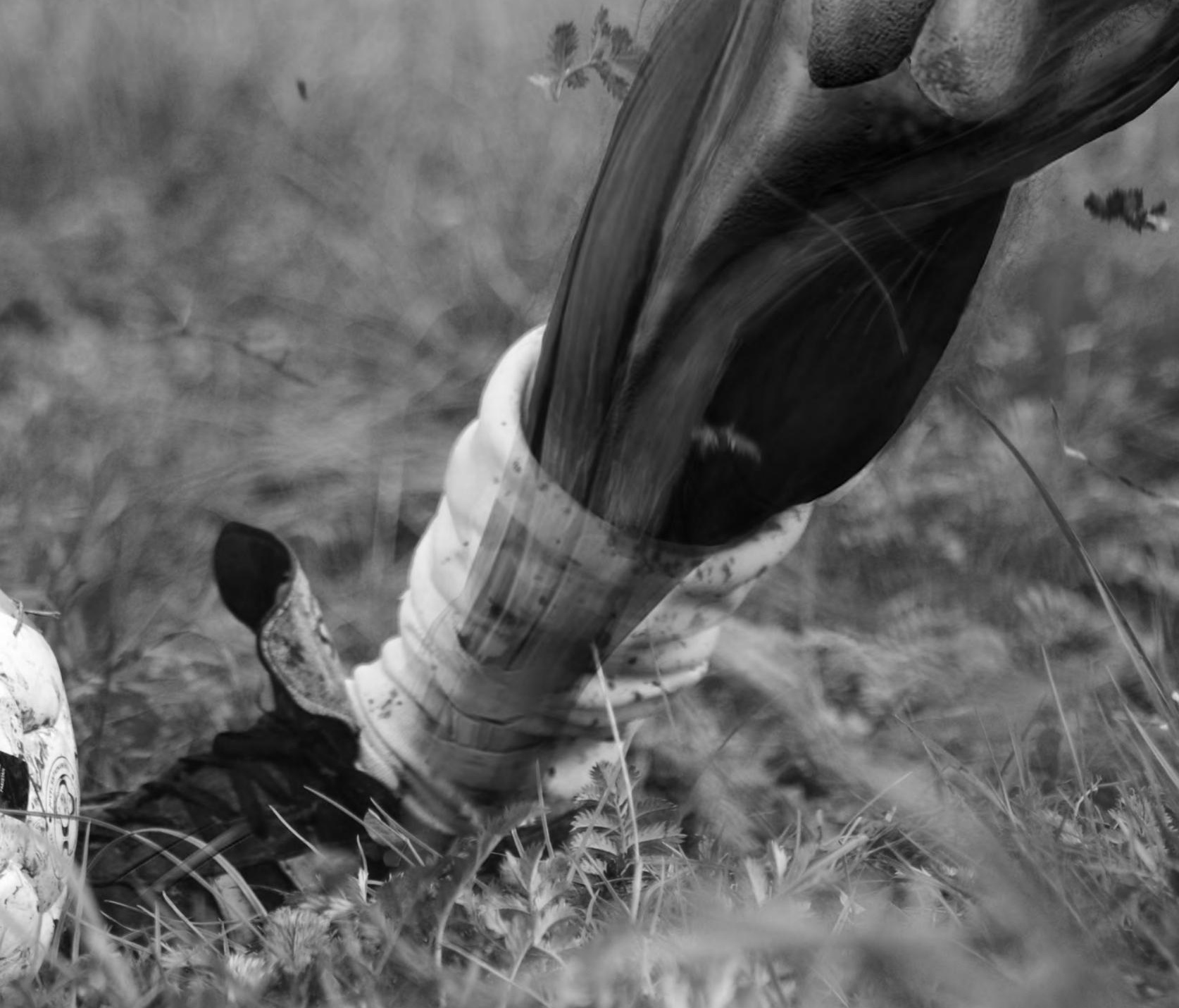




\section{CHAPTER 7}

\section{Assessing infection risk in implanted tissue-engineered devices}

R. Kuijer, E.J.P. Jansen, P.J. Emans, S.K. Bulstra, J. Riesle, J. Pieper, D.W. Grainger, H.J. Busscher 


\section{Abstract}

Peri-operative contamination is the major cause of biomaterial-associated infections, highly complicating surgical patient outcomes. While this risk in traditional implanted biomaterials is well-recognised, newer cell-seeded, biologically conducive tissue-engineered (TE) constructs now targeted for human use have not been assessed for this possibility. We investigated infection incidence of implanted, degradable polyester TE scaffold biomaterials in rabbit knee osteochondral defects.

Sterile, polyester copolymer scaffolds of different compositions and cellaccessible pore volumes were surgically inserted into rabbit osteochondral defects for periods of 3 weeks up to 9 months, either with or without initial seeding with autologous or allogeneic chondrocytes. Infection assessment included observation of pus or abscesses in or near the knee joint and postmortem histological evaluation.

Of 228 implanted TE scaffolds, 10 appeared to be infected: 6 scaffolds without cell seeding $(3.6 \%)$ and 4 cell-seeded scaffolds (6.3\%). These infections were evident across all scaffold types, independent of polymer composition or available pore volume, and up to 9 months.

We conclude that infections in TE implants pose a serious problem with incidences similar to current biomaterials-associated infections. Infection control measures should be developed in tissue engineering to avoid further complications when TE devices emerge clinically. 


\section{INTRODUCTION}

Microbial contamination is a potential risk of all surgical procedures, with clinical incidence variable, dependent upon many factors, including the operating facility, procedure, biomaterial implant and site, patient health, and pre- and postoperative care. Surgically implanted materials substantially increase patient predisposition to infection. Host defence is compromised considerably by the presence of an implanted foreign material. ${ }^{299-301}$ Local trauma and tissue morbidity around the implant site from surgical intervention, disrupted tissue perfusion, and subsequent protection offered to opportunistic colonizing organisms by the implant environment facilitate biofilm modes of pathogen growth on surgically placed biomaterials, protecting these organisms from host immune defences and antibiotics. Additionally, the consequences of implant-associated infections are more severe than for non-implant-associated infections: septic complications from surgical device placement often ultimately require surgical removal of the implant, with increased morbidity, and substantial, additional costs of care. Peri-operative contamination is considered to be the most common cause of biomaterial-associated infections ${ }^{299-301}$ in solid, non-degradable implants, such as hip or knee prostheses implanted into millions of patients annually.

Tissue engineering is increasingly advocated as a solution to many performance challenges associated with the restoration of human function using implanted biomaterials. ${ }^{131,302-304}$ Tissue engineering often requires highly porous, degradable biomaterial scaffolds which are, by nature, high surface area implant-grade natural or synthetic polymers with known incidences and histories for clinical infections. Tissue-engineered (TE) scaffold materials are implanted directly or after being seeded and cultured with viable cells, cell matrix proteins and growth factors most appropriate to the tissue application. ${ }^{131,304}$ This composite, 'living' device is surgically implanted to regenerate both tissue form and living function in vivo. Tissue engineered constructs represent a new case of surgically implanted biomaterials and, because they have been tailored to be more biologically conducive and integrating, might as such also be expected to be more susceptible to pathogen colonization as well. To date, no studies have been performed examining the occurrence of tissue engineering-based infection incidence in either preclinical or human clinical applications. Hence, the potential or extent of implantcentered infections in TE constructs is currently virtually unknown. 
Two decades ago, Gristina characterized biomaterial-associated infection as a "race for the surface", in which opportunistic pathogens from peri-operative contamination more readily adapt and colonize an implant surface over endogenous host cells to establish infection. ${ }^{305,306}$ Although the risk of perioperative microbial contamination might be considered identical between porous, degradable scaffolds and solid traditional implants, TE scaffolds preseeded with enabling matrix proteins and cells or tissues prior to implantation are essentially "pre-colonized" with cells, representing a potential barrier to subsequent bacterial colonization. Alternatively, high scaffold porosity and associated increased surface area offers pathogens increased surface area for attachment and possibly also a protective environment. Materials degradation may directly affect microbial adhesion as might the presence of cells or tissue products, but exact mechanisms are not yet explored in much detail.

In this study, we report infection rates for biodegradable polymer scaffolds used for tissue engineering of osteochondral defects in an accepted animal model. To this end, we retrospectively examined TE constructs in five different experiments for signs of infection. Data from over 200 examinations indicate that incidence of infection is similar to that for conventional knee implant prosthetic devices. 


\section{Materials AND Methods}

\section{Degradable Polymer Scaffolds}

TE polymer scaffolds were prepared from known degradable polyester copolymers containing blocks of poly(ethylene oxide terephthalate) (PEOT) and poly(butylene terephthalate) (PBT), (PEOT/PBT copolymers) previously characterized as biomaterials in different compositions and structures as described (Table 1). ${ }^{307,308}$ The nomenclature for PEOT/PBT polymers is as follows: "PEOT/PBT" a $\mathrm{b} / \mathrm{c}$, where

Table 1. PEOT/PBT scaffolds with or without cells implanted into osteochondral defects in rabbits.

\begin{tabular}{lccc} 
Scaffold ID & Cells & $\begin{array}{c}\text { Follow-up } \\
\text { (weeks) }\end{array}$ & $n$ \\
\hline CM 300 55/45 & - & 3 & 13 \\
& + & 3 & 4 \\
& - & 13 & 28 \\
& + & 13 & 36 \\
\hline CM 1000 60/40 & - & 39 & 8 \\
& + & 3 & 5 \\
& - & 4 & 15 \\
& - & 8 & 18 \\
CM 1000 70/30 & + & 13 & 6 \\
\hline P 300 55/45 & - & 26 & 18 \\
& - & 52 & 17 \\
\hline & - & 13 & 12 \\
\hline CM blend & - & 3 & 5 \\
& + & 3 & 7 \\
& - & 13 & 5 \\
& + & 13 & 5 \\
& - & 39 & 7 \\
\hline & - & 3 & 6 \\
& - & 13 & 7 \\
\hline
\end{tabular}

$a$ is the molecular weight of the poly (ethylene oxide) block starting compound, $b$ the weight percentage of the PEOT soft segments and $c$ the weight percentage of the PBT hard segments. These PEOT/PBT scaffolds have been comprehensively 
studied as biomaterials and their in vitro and in vivo performance published. ${ }^{159,160,}$ 308-318 In none of the studies in which these materials were surgically implanted was this copolymer found to be an irritant or to produce an aseptic purulent discharge. In addition to study of scaffolds of a single copolymer composition, blended scaffolds were also used, with a core of PEOT/PBT 300 55/45 and a surface of PEOT/PBT 1000 70/30. Scaffolds were prepared from the raw materials by compression moulding (CM) or by rapid prototyping (RP), as described and reviewed elsewhere (Figure 1). ${ }^{307,} 308$ These degradable porous polymer TE
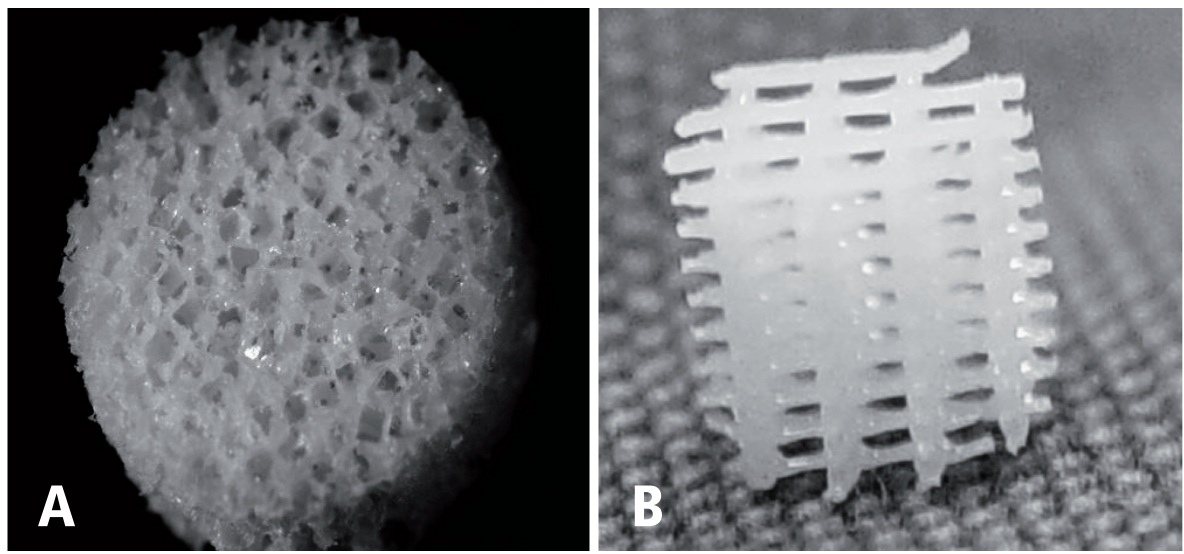

Figure 1. Photographs of PEOT/PBT scaffolds used in this study. (A): a PEOT/PBT 300 55/45 scaffold produced by compression moulding; (B): a PEOT/PBT 300 55/45 scaffold produced by 3 D printing technique.

scaffolds were manufactured at IsoTis Orthobiologics using principles of good laboratory practice (GLP) and good manufacturing practice (GMP). Scaffolds were $4 \mathrm{~mm}$ in diameter $\times 4 \mathrm{~mm}$ high with porosity of both CM and RP scaffolds of approximately $80 \%$ and average pore sizes of $182 \mu \mathrm{m}$ and $525 \mu \mathrm{m}$, respectively. ${ }^{319}$ The accessible pore volume (measure for interconnectivity using mercury porisometry) at a pore size of $200 \mu \mathrm{m}$ was $20 \%$ for the CM scaffold and $98 \%$ for the RP-scaffolds. Scaffolds were sterilized by $\gamma$-irradiation at $25 \mathrm{kGy}$ prior to either cell seeding or rabbit implantation.

\section{Isolation and seeding of chondrocytes}

Isolation, culture and seeding of rabbit chondrocyte cells for implant seeding were performed at IsoTis Orthobiologics under GLP and GMP conditions. Chondrocytes were isolated from articular cartilage harvested from 3 to 6 month-old New Zealand white rabbits used for other unrelated experiments 
(i.e., knee joints were naive). Articular cartilage was dissected from the knee and elbow joints and transported to IsoTis Orthobiologics Inc. (Bilthoven, NL) in transport medium (Dulbecco's modified Eagles Medium (DMEM) containing HEPES as buffer, $0.2 \mathrm{mM}$ ascorbic acid-2-phosphate (AA2P), $0.4 \mathrm{mM}$ L-proline, $0.1 \mathrm{mM}$ non-essential amino acids (NEAA), 100 units $/ \mathrm{mL}$ penicillin, $100 \mu \mathrm{g} / \mathrm{mL}$ streptomycin and $0.25 \mu \mathrm{g} / \mathrm{mL}$ amphotericin B). At IsoTis Orthobiologics the samples were digested overnight with $300 \mathrm{U} / \mathrm{mL}$ collagenase type II in HEPESbuffered DMEM for isolation of chondrocytes, and then expanded in monolayer cultures in culture medium (DMEM supplemented with $0.2 \mathrm{mM} \mathrm{AA2P}, 0.4 \mathrm{mM}$ L-proline, $0.1 \mathrm{mM}$ NEAA, 100 units $/ \mathrm{mL}$ penicillin, $100 \mu \mathrm{g} / \mathrm{mL}$ streptomycin, $0.25 \mu \mathrm{g} / \mathrm{mL}$ amphotericin B and either $10 \%$ autogenic serum (AS) or $10 \%$ fetal bovine serum (FBS)), in $2-3$ passages for 18 days. Cells $\left(1 \times 10^{6} /\right.$ scaffold $)$ suspended in culture medium were seeded onto scaffolds using spinner flasks as described previously. ${ }^{320,321}$

\section{In vivo osteochondral implantation procedures}

Over the past 5 years we have performed numerous preclinical animal studies to evaluate these degradable polyester scaffolds for tissue engineering of articulating joint surfaces. Over this time, a total of 228 polymer scaffolds have been implanted into standard osteochondral defects in rabbit knees. Of these, 165 sterile devices were implanted without cells (scaffold-only) and 63 were seeded with allogeneic or autologous chondrocytes (cell-seeded scaffolds) and then surgically implanted. A total of 122 control surgical defects were made, which were not implanted with biomaterials scaffolds.

For all animal experiments, approval of the local committee for animal experiments was obtained. The experiments were performed according to national and European laws for animal experiments, and were re-evaluated with respect to the occurrence of infection. In general, surgical implantations into NZW rabbits (5-6 months old at the time of surgery, weighing between 2.5 and $3.5 \mathrm{~kg}$ ) were performed under strict aseptic conditions. Rabbits were fasted for at least $12 \mathrm{~h}$ before surgery. General anaesthesia was induced by intramuscular injection of $35 \mathrm{mg}$ ketamine hydrochloride/kg body weight and $5 \mathrm{mg}$ xylazine/kg body weight and maintained by administration of $2 \%$ halothane and a mixture of oxygen and nitrous oxide delivered by an automatic ventilator using a specially designed mask. In later experiments anaesthesia was maintained with $2 \%$ isoflurane and oxygen. Pre-operatively all rabbits received an intramuscular injection of $10 \mathrm{mg} / \mathrm{kg}$ ceftiofur (Pharmacia \& Upjohn, Woerden, The Netherlands) to reduce the risk of per- or postoperative infections. 
The knee joints of the anaesthetized rabbits were carefully shaven and all fur was removed. Then the surgical site was sterilized using iodine solution and the nonsterile parts of the rabbit were covered with sterile drapes. All surgeons wore sterile coats and gloves. All instruments were sterilized and kept sterile during the operation procedures. An arthrotomy of the knee joint was performed through a medial longitudinal parapatellar incision. The medial capsule was incised and the patella laterally dislocated. The medial femoral condyle was exposed and a $4 \mathrm{~mm}$ diameter $\times 4 \mathrm{~mm}$ deep bore hole was created through the articulating surface into the subchondral bone using a low speed drill (100 - 150 rpm). Then the TE scaffold was press-fitted inside the osteochondral defect, the patella was repositioned, the capsule closed with Polysorb 2.0 (Tyco Healthcare, St. Louis, MO) taking care to approximate the medial capsule and extensor aperture to avoid patella luxation. The skin was closed with Polysorb 4.0 (Tyco Healthcare, St. Louis, MO). The wounds were subsequently disinfected with iodine solution once more. For each experimental group controls included sham-operated joints and empty osteochondral defects. Postoperative analgesia was administered ( $50 \mu \mathrm{g} / \mathrm{kg}$ buphenomorphine) at $2 \mathrm{~h}$ and 1 day.

Rabbits were sacrificed using an overdose of pentobarbital administered intravenously. The knees were opened and macroscopically judged for normal healing processes. Purulent infected joints (i.e. producing pus) were excluded from the original study to analyze further functional scaffold performance, but were enumerated for inclusion in the present infection study.

\section{Histological analysis}

The femoral condyles were dissected, photographed and then fixed in 10\% buffered formalin solution for 1 week. Then the samples were rinsed in running tap water for $1 \mathrm{~h}$ and decalcified in $10 \% \mathrm{EDTA} \mathrm{pH}=7.4$. Subsequently, the samples were dehydrated in a series of increasing concentrations of ethanol and embedded in 2-hydroxyethyl-methacrylate (Technovit 7100, Hereaus Kulzer, Wehrheim, Germany). Sagital sections of $4 \mu \mathrm{m}$ thick were prepared and stained with thionine or haematoxylin/eosin (H\&E). For this study the H\&E sections were examined for signs of severe infection (Figure 2). Sections showing mild inflammatory reactions were not assigned to the infected joints, but considered a result from the wound healing reaction (short follow-up), the presence of allogeneic cells or host foreign body reactions, which were stronger near scaffolds with higher percentages of PEOT. 

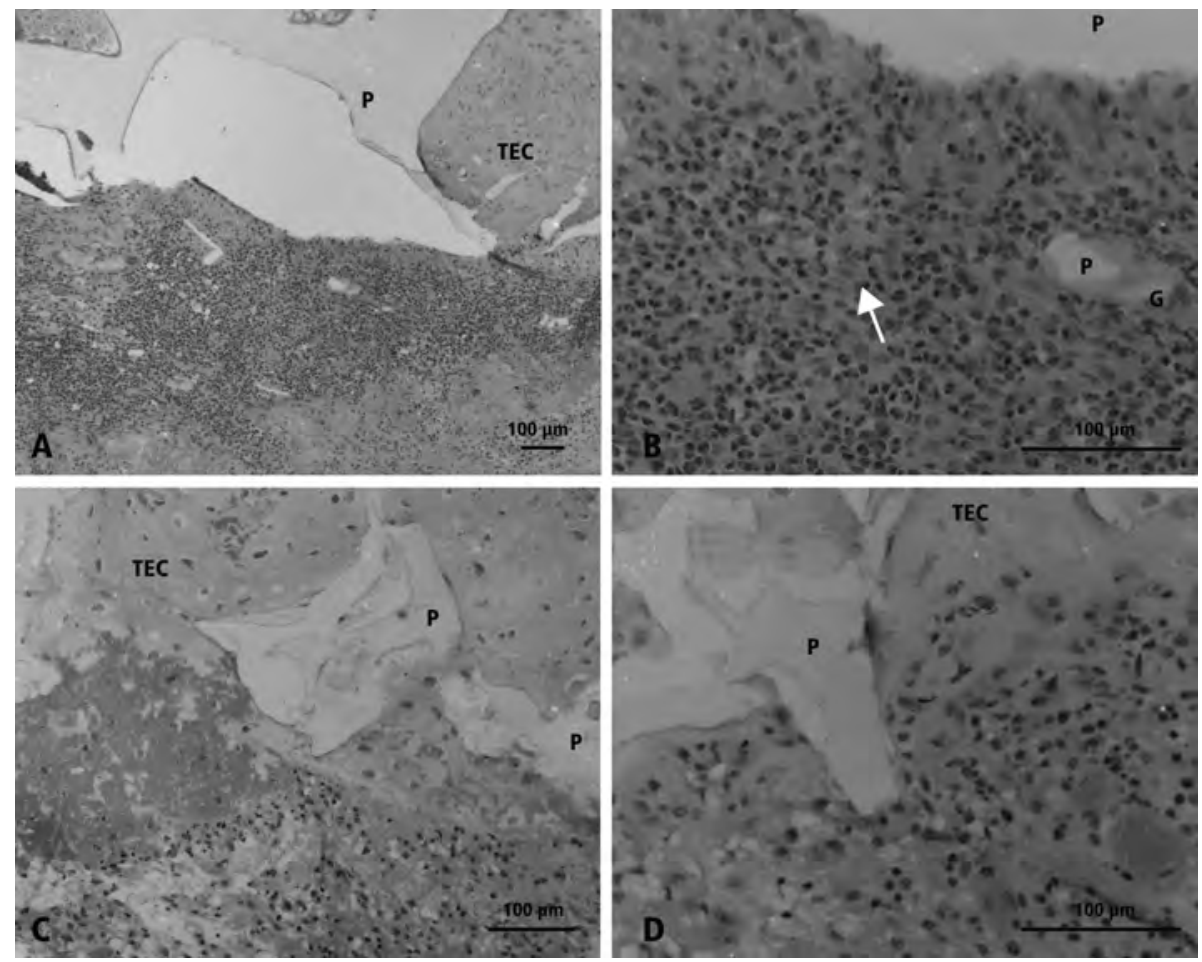

Figure 2. Histological observations of infected areas near an implanted PEOT/PBT 1000 60/40 scaffold with seeded and cultured allogeneic chondrocytes at 3 months follow-up. A - infected tissue with many polymorphonuclear cells and macrophages; $B$ - detail of $A$; $C$ - mild inflammatory reaction due to necrotic tissue-engineered cartilage; $\mathrm{D}$ - detail of $\mathrm{C}$ : limited number of polymorphonuclear cells present. P: Biomaterial, PEOT/PBT scaffold; G: giant cell; TEC: tissueengineered cartilage; Arrow: polymorphonuclear cell. (for full-colour figure see page 203)

\section{Criteria for clinical diagnosis of infection}

Infections were diagnosed in one of two ways:

1. Macroscopically - observations of purulent infected joints (i.e., producing pus) and

2. Microscopically - histological sections showing signs of severe inflammation with large fields of numerous inflammatory cells.

\section{Statistical analysis}

Differences between groups with infected scaffolds were analyzed manually using $\chi^{2}$ statistics. 


\section{ReSULTS AND DISCUSSION}

Of the 228 implanted scaffolds examined, a total of 10 were highly suspected for bacterial infection due to either pus (1) or severe inflammation from histological viewing (9). In the scaffold-only samples, 6 out of 165 were visibly infected $(3.6 \%)$ and in the cell-seeded scaffolds 4 out of $63(6.3 \%)$. The difference in infection rates between scaffold-only and cell-seeded scaffolds was statistically insignificant ( $\chi^{2}$ statistics). Infections were seen across all scaffold types, regardless of polymer composition (e.g. PEOT/PBT 300 55/45, PEOT/PBT 1000 60/40, PEOT/PBT 1000 70/30), blended formulations, or available pore volume in $\mathrm{CM}$ and PR scaffolds (Figure $3 \mathrm{~A}$ and $\mathrm{C}$ ). Infections were also found at most follow-up periods up to 9 months (Figure 3B and D). None of the 122 control-treated joints (sham, empty osteochondral defects) appeared to be infected.

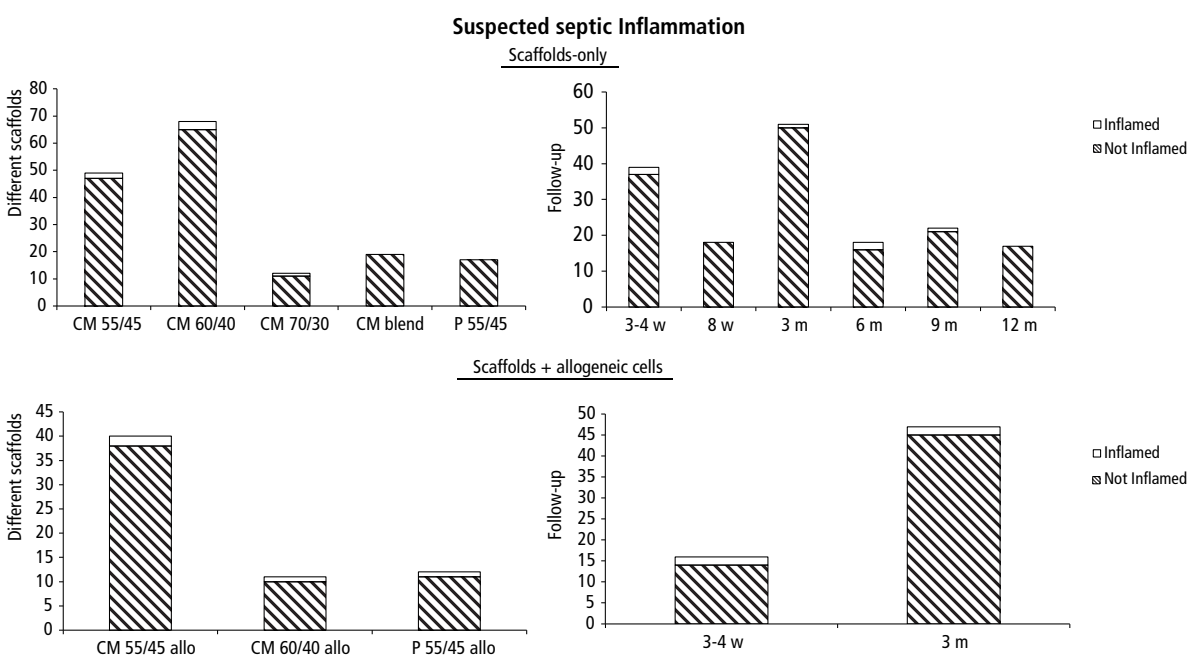

Figure 3. Inflammation incidence observed in PEOT/PBT scaffolds without cells (upper panel) and TE scaffolds with cells (lower panel) for different compositions (left panel) and different follow-up periods (right panel).

A retrospective survey of these osteochondral TE implants into articular joint surfaces revealed that observed infection rates are as significant a problem as those encountered in other surgically implanted biomaterials. ${ }^{322-326}$ Since infections were found at all follow-up times it is likely that infecting bacteria formed colonized, surface-resident biofilms on the porous degradable TE 
scaffolds, although it can not be excluded that planktonic bacteria also survive in the tissue both within and around a scaffold, similar to that described for pericatheter infections. ${ }^{326,327}$ Such microbial survival is assisted by local immuno-compromise common at implant sites, ${ }^{301}$ but it is also known that bacteria can remain dormant or extremely slow growing on implant surfaces for several months to years before manifesting pathogenic biofilms (small colony variants). ${ }^{328}$ Allogenic cell handling and sourcing, as well as the surgical insertion itself produce an increased risk of bacterial contamination, possibly contributing to the increased infection incidence observed in the cell-seeded cohorts.

We were forced to use two alternative methods for establishing bacterial infection other than culturing the contaminating bacteria, most often considered to be the "gold standard". Recent data suggest that this "gold standard" method often results in false negative results. ${ }^{329,} 330$ Since implantation of the PEOT/PBT copolymers has never produced aseptic purulent discharge to date, ${ }^{314-316,318,331-333}$ the presence of pus in a joint was considered to be definitive for microbial infection. Histological examination is more prone to false positive values, due to the fact that other, natural inflammatory processes are also present. In the short term experiments, the acute phase wound healing reaction makes interpretation of the histology difficult. In the long-term experiments, the foreign body reaction and also immunological reactions towards allogeneic-seeded cells or tissue produce acute and chronic inflammatory reactions. These confounding circumstances were eliminated by counting only highly infected samples, a conservative approach that may result in false negative results. Thus, the number of microbial infections may actually be higher.

New Zealand white rabbits were used for all implant experiments. Both infection resistance and inflammatory responses of rabbits undoubtedly differ from that of humans. Nevertheless, rabbits are often used as models for musculoskeletal infections, such as osteomyelitis ${ }^{334-338}$ and implant-centered infection studies ${ }^{339}$ and are considered a reliable, standard animal model for this problem. Implant-centered infection rates observed are roughly similar to those found in clinical settings for total hip and knee arthroplasties (approximately 1-3\%). ${ }^{322,} 340$ Furthermore, rabbit osteochondral infections can become chronic as indicated by infection presence at 9 months follow-up. These two observations relevant to human infection conditions support use of the rabbit with antibiotic prophylaxis during the surgery as a suitable model for this type of implant infection study. 
Additionally, reliable cell culture on these PEOT/PBT polymers is highly dependent on scaffold morphology, surface chemistry, cell seeding methodology and treatment with provisional matrix components to promote tissue and cell ingrowth. ${ }^{160,308,341,342}$ The same holds for most porous synthetic polymer scaffolds used for tissue engineering. ${ }^{33}$ Promotion of biologically conducive properties in these implants for improved host integration would be presumed to also enable bacterial colonization: cell-adhesive proteins are also bacterialadhesive $^{344}$ (e.g., collagen, fibrinogen, fibronectin, laminin), and cell culture media is also enabling to bacterial and fungal growth. Hence, TE scaffolds fulfilling host tissue integration requirements would also be expected to be more susceptible to infection, unless pre-colonization of TE surfaces with seeded cells was effective in the 'race for the surface' at inhibiting microbial post-surgical colonization. Some examples of seeded cell displacement or detachment from, or substantial cell necrosis on TE scaffolds post-implantation are known, ${ }^{319,}, 345$, 346 and attributed to the changing in vitro-in vivo environmental factors. As the osteochondral defect in the joint synovium has little vascular supply, TE scaffolds implanted into this local environment might be expected to have low $\mathrm{pO}_{2}$, suffer local acute hypoxia, as well as other acute adverse inflammatory responses from the surgical trauma that would adversely affect cell growth and provide a niche for microbial adhesion and colonization.

Biomaterial-associated infections in general are low incidence, but because of their extensive significance and increasing complications (i.e. antibiotic resistance) across all device categories, such infections represent a substantial total clinical case load annually, high cost burdens on the health care system for mitigation, and enormous patient discomfort and not infrequently, death. These factors make it difficult to clinically evaluate new effective measures to reduce such implant infections. Repeat studies on TE scaffold infections including over 200 in vivo implant cases is unlikely to occur frequently: the current results are the first reliable indications on infection occurrence in tissue engineering. Considering the cost and morbidity consequences of these infections, and the known difficulties in treating them effectively, these results should be considered alarming. New focus on infection control measures in tissue engineering should be instituted to avoid increased clinical complications when tissue engineering approaches enter the clinic in various forms.

\section{Conclusions}

TE scaffolds surgically implanted into osteochondral defects both with or 
without seeded or cultured cells are prone to microbial infection at the same rates, i.e. $6.3 \%$ and $3.6 \%$, respectively) as traditional biomaterials-based implants. Both acute and chronic inflammatory reactions consistent with infection were observed under conservative explant assessments. Infection data from these implant experiments carried out during the period from 2001 to 2005 involving 228 polymeric scaffolds are unique: no infection rates on TE devices have been reported to date. Yet, it is intuitive that this should be a problem intrinsic to these TE device categories: there is little difference in infection rates in cell-seeded versus non-seeded scaffolds, and nothing intrinsically unique in the TE construct should make these devices any more resistant to infection than other implanted biomaterials. 


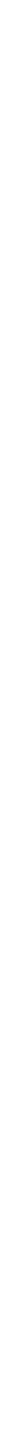




\section{CHAPTER 8}

\section{Hydrophobicity as a design}

criterion for polymer scaffolds in

bone tissue engineering

E.J.P. Jansen, R.E.J. Sladek, H. Bahar, A.Yaffe, M.J. Gijbels, R. Kuijer, S.K. Bulstra,

N.A. Guldemond, I.Binderman, L.H. Koole 


\section{Abstract}

Porous polymeric scaffolds play a key role in most tissue engineering strategies. A series of non-degrading porous scaffolds was prepared, based on bulkcopolymerization of 1-vinyl-2-pyrrolidinone (NVP) and n-butyl methacrylate (BMA), followed by a particulate-leaching step to generate porosity. Biocompatibility of these scaffolds was evaluated in vitro and in vivo. Furthermore, the scaffold materials were studied using the so-called demineralised bone matrix (DBM) as an evaluation system in vivo. The DBM, which is essentially a part of a rat femoral bone after processing with mineral acid, provides a suitable environment for ectopic bone formation, provided that the cavity of the DBM is filled with bone marrow prior to subcutaneous implantation in the thoracic region of rats. Various scaffold materials, differing with respect to composition and, hence, hydrophilicity, were introduced into the centre of DBMs. The ends were closed with rat bone marrow, and ectopic bone formation was monitored after 4, 6, and 8 weeks, both through X-ray microradiography and histology.

The 50 : 50 scaffold particles were found to readily accommodate formation of bone tissue within their pores, whereas this was much less the case for the more hydrophilic $70: 30$ counterpart scaffolds. New healthy bone tissue was encountered inside the pores of the 50:50 scaffold material, not only at the periphery of the constructs but also in the center. Active osteoblast cells were found at the bone-biomaterial interfaces.

These data indicate that the hydrophobicity of the biomaterial is, most likely, an important design criterion for polymeric scaffolds which should promote the healing of bone defects. Furthermore, it is argued that stable, nondegrading porous biomaterials, like those used in this study, provide an important tool to expand our comprehension of the role of biomaterials in scaffold-based tissue engineering approaches. 


\section{INTRODUCTION}

Porous polymeric scaffolds play a pivotal role in tissue engineering of bone and cartilage. ${ }^{347}$ To repair a bone or cartilage defect, one of the ideal scenarios can be summarised as follows: the patient's own osteoblasts, chondrocytes, or mesenchymal stem cells are harvested, expanded (in vitro), and seeded onto and in a scaffold (in vitro). The scaffold is then used to fill the defect cavity. In situ, the scaffold degrades slowly, as tissue growth proceeds towards complete filling of the defect. ${ }^{348}$ In an alternative scenario, a scaffold without cells is implanted directly into the defect cavity to serve as guidance for cell and tissue growth. ${ }^{349}$

The last years have seen continuous refinement and improvement of tissue engineering strategies, but a number of tough practical problems persists. ${ }^{350}$ This may have to do, in part, with the truly multidisciplinary nature of this field, which integrates knowledge from (i), polymer material properties; (ii), microand macrostructure of scaffolds; (iii), biomechanics; (iv), cell biology; (v), biocompatibility and host defence reactions; (vi), and surgery. Four practical problems can be identified:

1. The rate of degradation in vivo is often difficult to control. This holds particularly true for poly ( $\alpha$-hydroxy acids) such as poly (lactic acid) and poly (glycolic acid), which show burst-degradation.

2. Efficiency of cell seeding is usually low. In many cases cells are found close to the surface of the scaffold, but not in the interior. ${ }^{351}$

3. The breakdown products formed during degradation of the scaffold may be cytotoxic. They may also invoke a local inflammatory response. For example, degradation of poly (lactic acid) can result in acidic building blocks that may elicit a local inflammatory response. This risk is especially high when there is little vascularisation, i.e. slow drainage of waste products from the implant site. ${ }^{352,353}$

4. Cells could dedifferentiate when seeded onto scaffolds. Chondrocytes, for example, have a well-known tendency to dedifferentiate after being seeded into polymeric scaffold structures in vitro. ${ }^{354}$

It is clear that our fundamental understanding of the role of the scaffold biomaterial is still rather limited. It has been argued already that it is necessary to improve the biomaterials in such a way that the scaffolds accommodate cell proliferation and deposition of extracellular matrix throughout their entire volume. Surface modification, for instance with the adhesive protein fibronectin, 
represents a promising strategy. ${ }^{355}$ Furthermore, in depth investigations are necessary to find out how de-differentiation of seeded cells can be prevented (complication \# 4).

A possible way to expand our understanding of scaffold materials may be to dissect the various factors that determine its ultimate success. One approach is to study scaffolds which do not decompose. Evaluation of the performance of such scaffolds, both in vitro and in vivo, may shed new light on the importance of the choice of the material. Such information can easily be obscured when degradable scaffolds are used, for example when decomposition products are cytotoxic for cells inside, or in the proximity of, the scaffold.

In this paper, we report the results of a study on a series of new porous polymeric scaffolds, which have in common that the biomaterial is stable, i.e. no degradation occurs. We prepared scaffolds from 1-vinyl-pyrrollidinone (NVP, a hydrophilic reactive monomer) and $n$-butyl-methacrylate (BMA, a hydrophobic reactive monomer). ${ }^{356-358}$ Our choice of NVP-BMA copolymers was based on our previous experience with biocompatibility of these materials. ${ }^{359}$ Two series of scaffolds were studied, one is relatively hydrophilic, with composition NVP : $\mathrm{BMA}=70: 30$ (mole: mole), and one is more hydrophobic, with composition NVP : BMA = $50: 50$ (mole: mole). These scaffolds were compared on the basis of in vitro and in vivo experiments on biocompatibility and ectopic bone formation experiments. ${ }^{360}$

\section{MAterials AND METHOdS}

\section{Scaffold preparation}

All solvents and starting reagents were of the highest purity available, or purified as specified. 1-Vinyl-2-pyrrolidone (NVP) and $n$-butyl-methacrylate (BMA) were purchased from Acros Organics ('s-Hertogenbosch, The Netherlands). Prior to synthesis, the monomers were purified by distillation under reduced pressure. Purity was checked through nuclear magnetic resonance (NMR) spectroscopy. The radical initiator 2,2'-azobis(2-methylpropionitrile) (AIBN) was purchased from Aldrich (Aldrich Chemical Co. Inc., Milwaukee WI, USA), and used as received.

All materials were prepared according to the following procedure. NVP and $\mathrm{BMA}$ were mixed in the appropriate ratios, such that approx. $10 \mathrm{~g}$ of monomer mixture was obtained. AIBN was added to a concentration of 0.2 mole $\%$ of total monomer. The mixtures were homogenised in an ultrasonic bath for 5 min until the AIBN was dissolved completely. Then the monomer mixture was transferred into poly-tetrafluoroethylene tubes (inner diameter $=8 \mathrm{~mm}$, wall thickness $=1 \mathrm{~mm}$, length $=20 \mathrm{~cm}$ ), which were closed by a glass stopper 
on one end. The tubes were immersed in a thermostated oil bath, which was interfaced with a time-temperature control system as described previously. ${ }^{361}$, 362 The polymerizations afforded hard opaque rods, which could easily be removed from the Teflon ${ }^{\circledR}$ tubes. The top and bottom ends (approx. $1 \mathrm{~cm}$ ) were cut off and discarded. The copolymer rods were machined into pieces (approx. $500 \mathrm{mg}$ each), which were dissolved in chloroform at a concentration of $10 \%$ by weight. Under continuous mechanical stirring at room temperature (fume hood) the pieces completely dissolved in 2 days.

Sodium chloride crystals (MERCK) were separated through sieving (Analysensieb, DIN-ISO 3310/1, Retsch, Germany) such that three fractions were obtained: 200 to $300 \mu \mathrm{m}, 300$ to $425 \mu \mathrm{m}$, and greater than $425 \mu \mathrm{m}$. The salt crystals were added to the copolymer-solvent solution such that ratios salt (g) : copolymer $(\mathrm{g}): \mathrm{CHCl}_{3}(\mathrm{~mL})=9: 1: 10$ and $8: 2: 10$ were obtained. Resulting viscous slurries were poured into a glass beaker and stirred continuously with a spatula. Meanwhile the chloroform was allowed to evaporate (fume hood), which gradually increased the viscosity of the slurry. The material was then cast into a Teflon ${ }^{\circledR}$ mould that consisted of four cylindrical holes with diameter 20 $\mathrm{mm}$, and depth $4 \mathrm{~mm}$. Some experience in determining the right time of casting was required. Casting too early (viscosity too low) resulted in precipitation of the salt crystals to the bottom, leaving an inhomogeneous disk. Casting too late (viscosity too high) would make it impossible to cast the slurry into the moulds. Remnants of chloroform were allowed to evaporate for 2 days at room temperature in a fume hood, followed by vacuum drying in a dessicator for $2 \mathrm{~h}$. After evaporation of the chloroform, the copolymer-salt disks were removed from the moulds and wetted in nuclease-free sterile water for $30 \mathrm{~min}$. A biopsy punch was used to cut the scaffolds (diameter $4 \mathrm{~mm}$, height $4 \mathrm{~mm}$ ). The salt was leached out completely by immersing the scaffolds in nuclease-free sterile water on a shaker for 1-2 days. During this time period, the water was changed three times a day. The scaffolds were air-dried in a laminar airflow chamber for $48 \mathrm{~h}$ and sterilized using ethylene oxide (EtO) gas following a standard protocol. The samples were degassed for a minimum of $48 \mathrm{~h}$ in air, and for 3 days in a dessicator under vacuum. It is our experience that treatment with EtO gas is the preferred method of sterilisation for polymeric biomaterials.

\section{Material characterisation}

Purity of the scaffold copolymers was determined through ${ }^{1} \mathrm{H}$ nuclear magnetic resonance (NMR) spectroscopy. All scaffolds dissolved completely in deuterated chloroform. Spectra were recorded at $400 \mathrm{MHz}$ on a Varian UnityPlus spectrometer. 
The glass transition temperatures $(\mathrm{Tg})$ of the copolymers were measured on a Perkin-Elmer DSC (differential scanning calorimetry) at a heating rate of 10 centigrades/min.

Scaffold morphologies were examined by scanning electron microscopy (SEM), using a Personal SEM (R.J. Lee Instruments Ltd., USA). Samples were prepared by cutting the scaffolds in half perpendicular to their longitudinal axis. They were mounted on aluminium stubs and gold-coated using a sputter coater (BioRad SC500) set at $20 \mathrm{~mA}$ for a total of $2 \mathrm{~min}$. The instrument was set to 15 $\mathrm{kV}$ and the samples were oriented at an angle such that the inside of the scaffolds could be viewed.

\section{Biocompatibility in vitro}

Biocompatibility in vitro was studied by MTT-assay using mouse fibroblasts (3T3 cells, subclone CCL-92 from the American type culture collection) and direct contact methods using isolated rat calvarial bone cells and rat bone marrow cells.

In the MTT-experiment, cell viability was estimated after cell culturing in scaffold extract compared to latex extract $(1610 \mathrm{mg}$ latex (cytotoxic) in $8 \mathrm{~mL}$ medium) or medium. Prior to extraction in medium the latex was sterilized by immersing the latex in ethanol $70 \%$ during $30 \mathrm{~min}$. Then the latex was dried in an air flow cabinet. Extracts were prepared by extracting 10 scaffolds (porosity $80 \%$, pore size $200-300 \mu \mathrm{m}$ ) at $37^{\circ} \mathrm{C}$ for 4 days in $8 \mathrm{~mL}$ culture medium using a shaker.

Culture medium was Dulbecco's Modification of Eagle's medium/F12 nutrient mix (1:1) with L-Alanyl-L-Glutamine supplemented with pyridoxine (Gibco, UK), 10\% Fetal Calf Serum and 1\% penicillin/streptomycin/amphotericin. $3 \mathrm{~T} 3$ cells were seeded in 96-well plates $\left(\operatorname{Costar}^{\circledR}\right.$, Corning, USA) at a density of approx. 1000 cells/well in culture medium at $37^{\circ} \mathrm{C}$ and $5 \% \mathrm{CO}_{2}$ in an incubator overnight. The undiluted and diluted (10X) scaffold extracts were added to the cells.

After 3 days the extracts were replaced with culture medium containing (4,5-dimethylthiazol-2yl)-2,5-diphenyl-2H-tetrazolium bromide (MTT) $(600 \mu \mathrm{g}$ $\mathrm{MTT} / \mathrm{mL}$ culture medium). Cells were cultured for another $4 \mathrm{~h}$. Subsequently, the medium was discarded and the precipitated formazan was dissolved in DMSO $(60 \mu \mathrm{L} /$ well). The plates were gently shaken for 1-2 min. The absorbance at $550 \mathrm{~nm}$ was measured on a microplate reader.

The raw data from the MTT experiments were statistically treated in a nonparametric test. For the number of pair wise comparisons the Bonferroni correction was used. ${ }^{363}$ An $\alpha$-level of 0.00416 was considered as significantly different. 
Calvarial bone cell attachment and differentiation in direct contact to scaffolds was examined. Calvarial cells were obtained from calvarial bones of 17-19 days old Dark-Agouti (DA) rat embryos. The calvarial bone was carefully dissected from adhering tissues and washed twice in cold phosphate-buffered saline (PBS) solution at room temperature. Subsequently the calvarial bones were cut into small pieces and digested in trypsin-EDTA solution (16-24 calvarial bones in 45 $\mathrm{mL}$ digestion solution) in a $7 \% \mathrm{CO}_{2}$ humidified incubator for $20 \mathrm{~min}$ at $37^{\circ} \mathrm{C}$. Cell pellets were formed by centrifuging the supernatant at $1500 \mathrm{rpm}$ for $10 \mathrm{~min}$ $\left(4^{\circ} \mathrm{C}\right)$. Then the cell pellet was suspended in culture medium (Dulbecco's Modified Eagle's Medium (Biological Industries, Kibbutz Beit Haemek, Israel) supplemented with $10 \%$ fetal calf serum) to a concentration of 50,000 cells $/ \mathrm{mL}$. Scaffolds (porosity $80 \%$, pore size greater than $425 \mu \mathrm{m}$ ) were rinsed with PBS and degassed in a sterile filter unit (25944-500, $0.22 \mu \mathrm{m}$ Nylon, Corning, USA) before use. Subsequently in each $60-\mathrm{mm}$ culture dish (Falcon) one single scaffold and $4 \mathrm{~mL}$ of cell containing medium was dispersed. Culture dishes without scaffold material were used as control groups. The cells were allowed to attach onto the scaffold for 3-4 days. The medium was replaced by culture medium and changed each 4-6 days. After 25-30 days the scaffolds were harvested, fixed and stained with Alizarin-red for histological examination.

Rat bone marrow cell attachment and differentiation in direct contact with scaffolds was also studied. Eight $70: 30$ (NVP : BMA) and eight $50: 50$ scaffolds were used (porosity $80 \%$, pore size $>425 \mu \mathrm{m}$ ). Scaffolds were rinsed with sterile PBS and degassed in a filter (25944-500, $0.22 \mu \mathrm{m}$ Nylon, Corning, USA). Bone marrow cells were obtained by puncturing the femurs removed from 2-month-old DA rats with a $1.2 \mathrm{~mm}$ blunt needle. Cells of one femur were dispersed in each $60-\mathrm{mm}$ dish containing one single scaffold. Cells were allowed to attach for $30 \mathrm{~min}$. Then $4 \mathrm{~mL}$ of culture medium (Dulbecco's Modified Eagle's Medium + 4,5 g/l D-Glucose (Biological Industries, Kibbutz Beit Haemek, Israel) supplemented with $10 \%$ fetal calf serum + L-Glutamine + penicillin/streptomycin $+1-1.2 \mathrm{mM} \mathrm{Ca}^{2+}$ ) was added. The medium was changed every 2-3 days. Subsequently the cells were cultured in a humidified incubator at $37^{\circ} \mathrm{C}, 7 \% \mathrm{CO}_{2}$, and prepared for histological examination at 9 and 23 days follow-up respectively.

\section{Biocompatibility in vivo}

Biocompatibility in vivo was studied by implanting scaffolds subcutaneously in rats and by incorporating scaffolds in ectopic bone formation in rats using the demineralised bone matrix cylinder model. 
In the first in vivo study scaffolds were implanted subcutaneously in rats. The Maastricht University Committee for animal experiments approved the rat experiment protocols. The experiments were conducted following the national and European guidelines for animal experiments.

A total of 8 Lewis rats (age $10-12$ weeks) was used. In each rat a $50: 50$ scaffold and a $70: 30$ (NVP : BMA) scaffold were implanted. All scaffolds had a porosity of $80 \%$ and pore sizes of $200-300 \mu \mathrm{m}$.

The rats were fasted overnight. General anaesthesia was induced by subcutaneous injection of ketamine hydrochloride (100 $\mu \mathrm{L} / 100 \mathrm{~g})$ and xylazine $(50 \mu \mathrm{L} / 100 \mathrm{~g})$. The back was shaved, disinfected with $2 \%$ iodine solution and sterilely wrapped. Under rigorous aseptic conditions subcutaneous pouches were made in the back of the rat, in which the materials were implanted. The incisions were closed with Polysorb ${ }^{\circledR} 4.0$ or closed with clips. The animals were examined daily for signs of wound infection, behaviour abnormalities or illness.

After 7 or 84 days the rats were sacrificed. The implants were located and harvested with the surrounding tissue. After examination for signs of infection the explants were photographed and prepared for further evaluation. The specimens were fixed in $10 \%$ formalin solution during 3-5 days at $4^{\circ} \mathrm{C}$. Excessive fixative was rinsed with tap water for $1 \mathrm{~h}$. Samples were then gradually dehydrated in an ethanol series at room temperature, and embedded in 2hydroxyethyl methacrylate (Technovit ${ }^{\circledR} 7100$, Heraeus Kulzer GmbH\&Co., Darmstadt, Germany). The resin was left to harden, according to the manufacturer's instructions. Blocks were cut along the midsagittal plane into 4$\mu \mathrm{m}$ thick sections by means of a microtome (Leica RM 2065) and stained with Gill's haematoxylin/eosin. Sections were evaluated using light microscopy.

In the second in vivo study scaffolds participated in ectopic bone formation in rats, induced by the implantation of demineralised bone matrix (DBM) cylinders containing rat bone marrow cells and scaffold parts (vide infra). In previous studies ectopic bone formation in young rats was seen when bone marrow cells were inserted into the DBM model. ${ }^{360}$

Three-month-old DA rats were sacrificed and their femurs were harvested. Soft tissue and metaphyses were carefully removed in such a way that only cortical bone was left. Then diaphysic cylinders of approx. $7 \mathrm{~mm}$ length were prepared. Subsequently the bone cylinders were treated with $0.6 \mathrm{M} \mathrm{HCl}$ for 24$48 \mathrm{~h}$. The resulting DBMs were rinsed with distilled water and kept in 70\% alcohol. Prior to implantation, alcohol was washed out with sterile PBS.

Scaffolds, $50: 50$ and $70: 30$ (NVP : BMA; porosity $80 \%$ and $90 \%$, pore size greater than $425 \mu \mathrm{m}$ ) were cut into pieces of approx. $1 \times 1 \times 3 \mathrm{~mm}$, because 
the original scaffold dimensions were larger than the dimensions of the DBM cylinders. Fresh bone marrow cells were obtained from femurs of 2-month-old DA rats using a $1200 \mu \mathrm{m}$ blunt needle. Scaffold particles were placed in the centre of the DBM cylinders and the fresh bone marrow cells were immediately transferred into the DBM cylinders. DBM cylinders with fresh bone marrow cells, but without scaffold particles served as a positive control for bone formation. DBM cylinders with scaffold particles, but without fresh bone marrow cells served as negative controls.

Two-month-old DA rats were fasted overnight. General anaesthesia was induced by ketalar (Malgene 1000 Rhone Merieux, Lyon, France) and xylazine (Rampun Bayer, Leverkusen, Germany). The upper thoracic region was shaved, disinfected with iodine solution, and sterily wrapped. A 1-cm incision was made over the midline of the chest and subcutaneous tissue plains dissected laterally to form pouches in which the cylinders were implanted. Wounds were closed with silk 3.0 and washed again with iodine solution.

Animals were sacrificed after 4,6 or 8 weeks. The DBM cylinders were harvested and treated with $10 \%$ buffered formalin solution. Subsequently they were analyzed microradiographically using a Hewlett Packard Faxitron Cabinet. Specimens were exposed for 5 seconds at $25 \mathrm{KeV}$ using Kodak Ektaspeed E safety film. ${ }^{364,365}$ After microradiographic analysis, the cylinders were demineralised in EDTA and stained with haematoxylin/eosin. The sections were evaluated for bone apposition and bone ingrowth onto the scaffolds.

\section{RESULTS AND DISCUSSION}

Synthesis of the copolymers proceeded without difficulties. Our NMR spectra of both copolymers, measured directly after their synthesis, confirmed the presence of unreacted NVP (approx. 1\% of the original amount of NVP), while unreacted BMA could not be detected (data not shown). It is important to recall that NVP is known to be much less reactive as compared to methacrylates. ${ }^{366}$ Consequently, BMA is consumed faster than NVP during copolymerization. It is also known that diffusion limitation towards the end of the reaction leaves unreacted NVP, as well as relatively short NVP homo-oligomers within the material. Different mixtures of NVP and BMA yield copolymers with markedly different hydrophilicities, also after complete release of extractables. Water uptake for the 70 : 30 (NVP : BMA) copolymer was found to be $150 \%$; for the 50 : 50 (NVP : BMA) copolymer this was approx. 50\%. ${ }^{367}$ Figure 1 shows a representative example of these spectra, which confirmed the identity and purity of our materials. It should be noted that the presence of extractable oligomers is 
difficult to establish from the NMR spectra. Monomers, on the other hand, can easily be detected, even if their concentration is as low as $0.05 \%$. In particular, we focused on the spectral region $\delta 7.2-4.6$. The presence of unreacted monomer results in signals in this region (i.e., $=\mathrm{CH}_{2}$ protons of NVP as a fourline pattern centered at $\delta 7.10$ and $=\mathrm{CH}_{2}$ protons of BMA at $\delta 6.10$ and 5.54). Even at large vertical expansion (inset in Figure 1), these peaks could not be detected. This made us conclude that all unreacted BMA, NVP and NVP homooligomers were effectively removed. Differential scanning calorimetry experiments showed clear glass transitions, with $\mathrm{Tg}=50^{\circ} \mathrm{C}$ for the $50: 50$ material and $58^{\circ} \mathrm{C}$ for $70: 30$ (NVP : BMA).
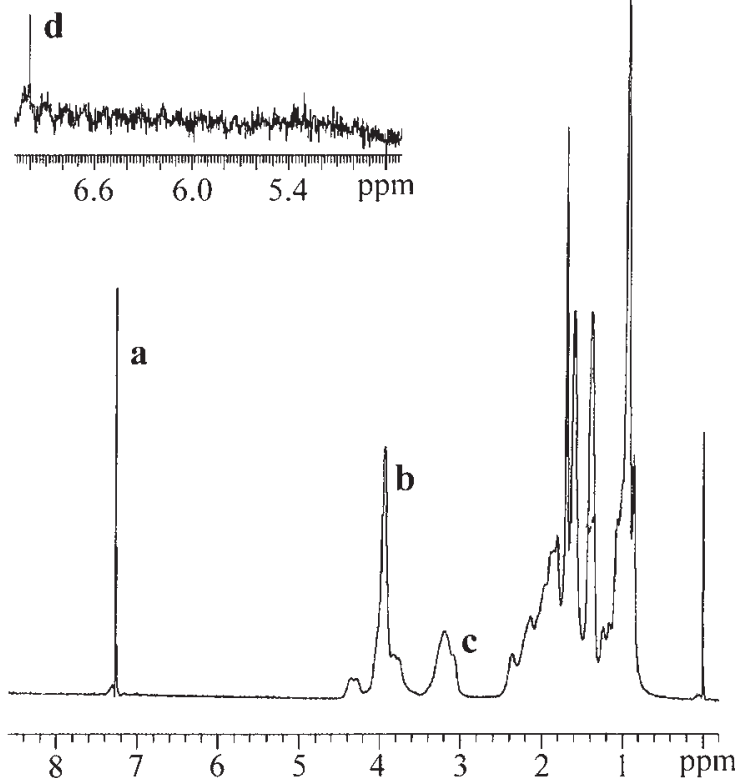

Figure 1. ${ }^{1} \mathrm{H}$ NMR spectrum of the $50: 50$ scaffold material, dissolved in deuterated $\mathrm{CDCl}_{3}$. Peak (a) corresponds with traces of $\mathrm{CHCl}_{3}$ in the solvent, peak (b) corresponds with the $\mathrm{O}-\mathrm{CH}_{2}$ - protons of the BMA blocks; peak (c) corresponds with the $\mathrm{N}-\mathrm{CH}_{2}$ - protons within the pyrrolidone ring of NVP blocks. Overlapping signals, arising from all other protons in the copolymer, are seen in the spectral region $\delta 2.6-0.8$. The inset shows the spectral region $\delta 7.1-4.8$, after $40 x$ vertical expansion. No resonances due to unreacted BMA or NVP are found. Peak (d) is the ${ }^{13} \mathrm{C}$ satellite of the protons in $\mathrm{CHCl}_{3}$. 
Figure 2 shows SEM micrographs of scaffolds that were prepared from the 70 : 30 (NVP : BMA) copolymer and sodium chloride in the mass: mass ratio $1: 9$ respectively (vide supra). Hence, these structures have approx. $10 \%$ of their
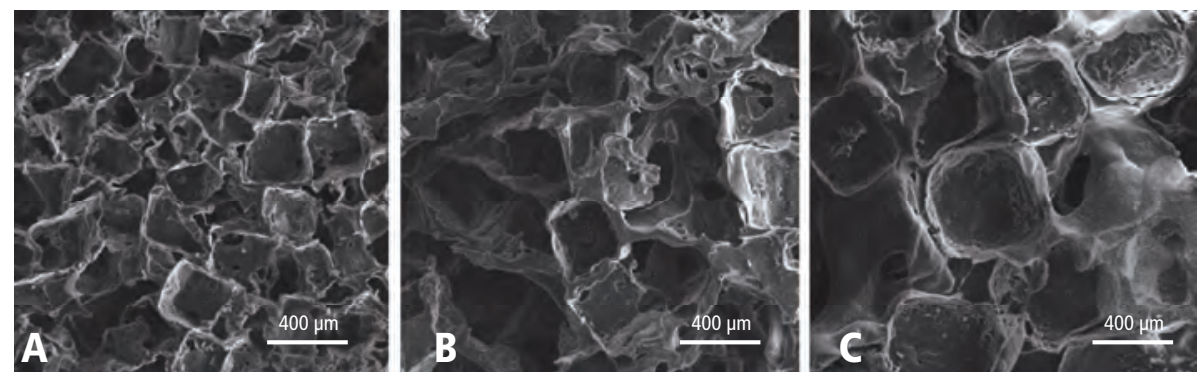

Figure 2. Representative SEM micrographs of dry $70: 30$ (NVP : BMA) scaffolds with porosity $90 \%$. These were fabricated through solvent casting and particulate leaching, as described in the Materials \& Methods section. Control over the pore size was obtained by varying the size of the salt crystals. A: scaffold prepared with salt particle sizes in the range: $200-300 \mu \mathrm{m}$. B: scaffold prepared with salt particle sizes in the range $300-425 \mu \mathrm{m}$. C: scaffold prepared with salt particles $>425 \mu \mathrm{m}$.

volume filled with copolymer, and $90 \%$ with pores. This resulted in an open pore structure in all cases. The highly porous nature resulted in clear interconnectivity of the pores. This is an essential feature, as interconnectivity is mandatory to facilitate ingrowth of cells.

Figure 3 summarizes the raw data of the MTT cytotoxicity tests. Apparently, the different undiluted extracts increase in toxicity in the order: negative control $<50: 50<70: 30<$ latex. All differences are statistically different $(p \leq 0.001)$. In all cases cytotoxicity decreases upon 10-fold dilution. Then, cytotoxicity changes as follows: negative control $\approx 50: 50 \approx 70: 30<$ latex. The $p$-values are: $50: 50$ versus negative control: $p=0.050$ (ns); $70: 30$ vs. negative control: $p=0.065$ (ns); $50: 50$ versus $70: 30: p=0.878$ (ns); 50 : 50 versus latex: $p=0.000$ (s) and $70: 30$ versus latex: $p=0.000$ (s).

The MTT experiments prompted us to study the scaffold materials in direct contact with cells. Rat calvarial bone cells and rat bone marrow cells were used. Figure 4 shows calvarial bone cells after 30-day incubation with $50: 50$ and 70 : 30 (NVP : BMA) materials. Cells were stained with Alizarin-red. It could be concluded that the cells proliferated in contact with both materials. Interestingly, some bone nodules were also observed. The presence of these bone nodules, including their mineralization, underlines the biocompatibility of both materials. 


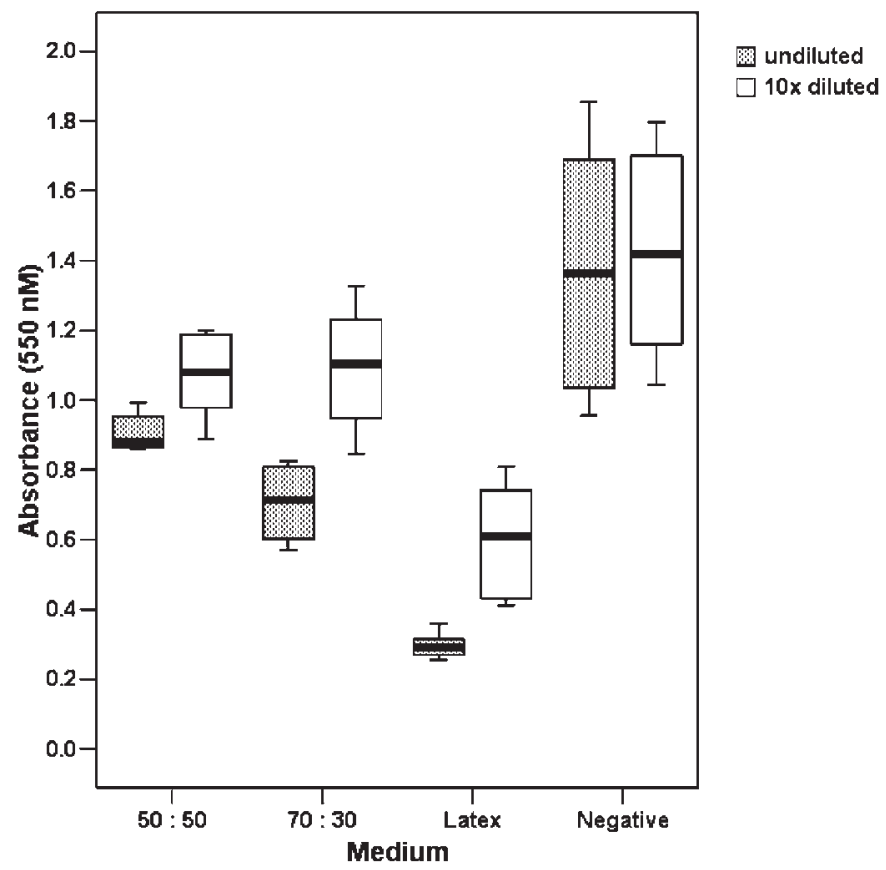

Figure 3. Mean absorbance as measured in the MTT test with fibroblast cells, incubated in undiluted extracts, and 10-fold diluted extracts. Extracts from latex rubber were used as a positive control. Error bars designate means plus/minus standard deviation for $n=8$, except for undiluted $50: 50(n=7)$, and undiluted $70: 30(n=6)$.

Furthermore, we evaluated the behaviour of primary rat bone marrow cells cultured in close contact with $70: 30$ (NVP : BMA) and $50: 50$ scaffolds for up to 23 days. The scaffolds had no negative influence on cell morphology, cell viability and proliferation. Nevertheless, cell differentiation was not seen after 9 and 23 days. Figure 5 shows a representative micrograph of rat bone marrow cells cultured in close contact with a $70: 30$ (NVP : BMA) scaffold. No bone nodules and/or mineralization were observed. Instead, de-differentiation of the bone marrow cells towards fibroblast-like cells seemed to have occurred.

Based on the combined data of our in vitro biocompatibility tests, we decided to do a series of in vivo experiments. In the first series, scaffolds were implanted subcutaneously on the back of 8 rats. Each animal received 2 scaffolds, i.e. one $50: 50$ and one $70: 30$ (NVP : BMA) scaffold. All scaffolds had a porosity of $80 \%$ and pore sizes of $200-300 \mu \mathrm{m}$. Follow-up was 7 days (4 animals) or 84 days (4 animals). 

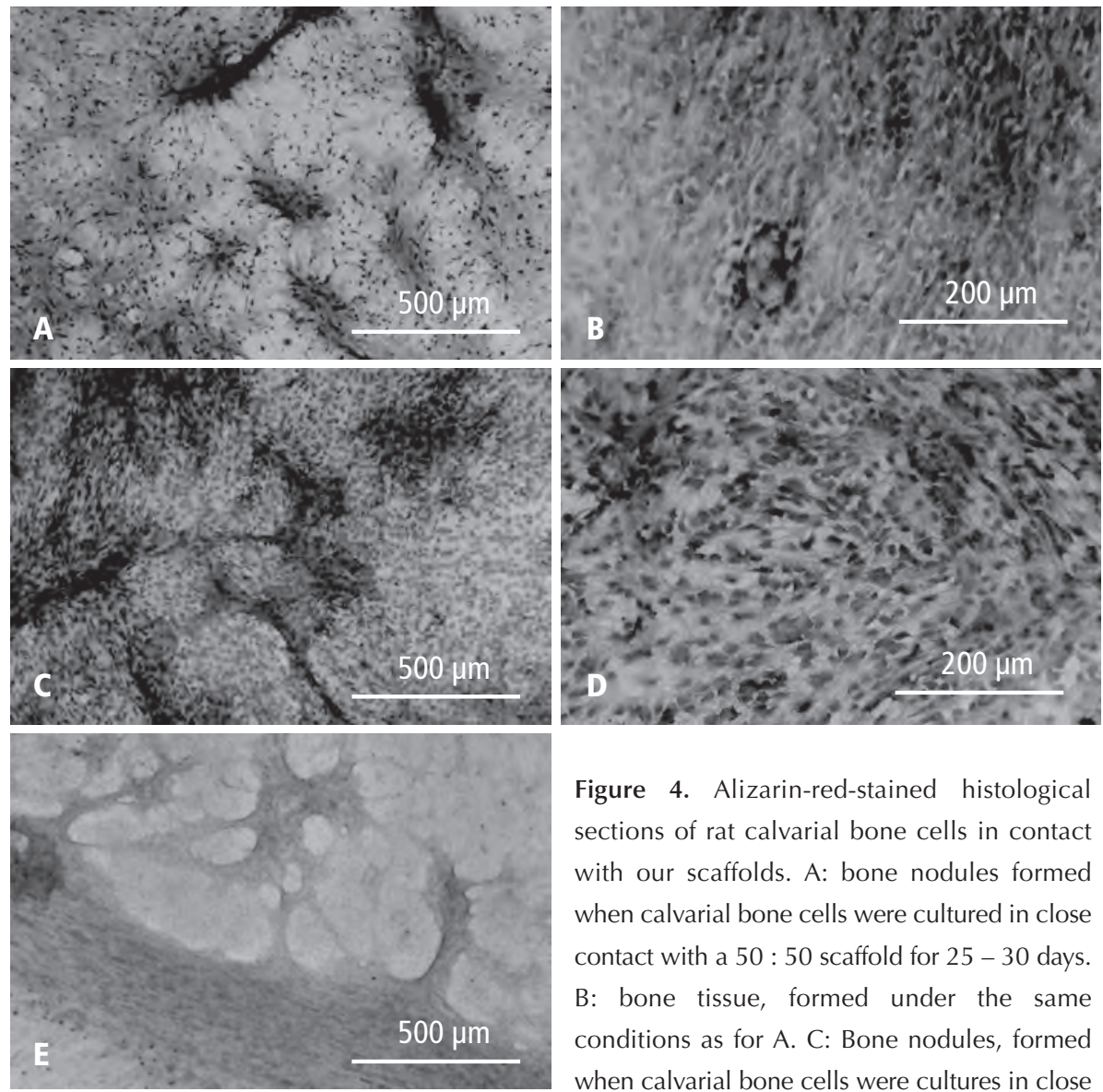

Figure 4. Alizarin-red-stained histological sections of rat calvarial bone cells in contact with our scaffolds. A: bone nodules formed when calvarial bone cells were cultured in close contact with a 50 : 50 scaffold for 25 - 30 days. B: bone tissue, formed under the same conditions as for A. C: Bone nodules, formed when calvarial bone cells were cultures in close contact with a $70: 30$ scaffold for 25 - 30 days. D: Cubical cells, formed under the same conditions as for C. E: Cell attachment onto the 50 : 50 scaffold. (for full-colour figure see page 204)

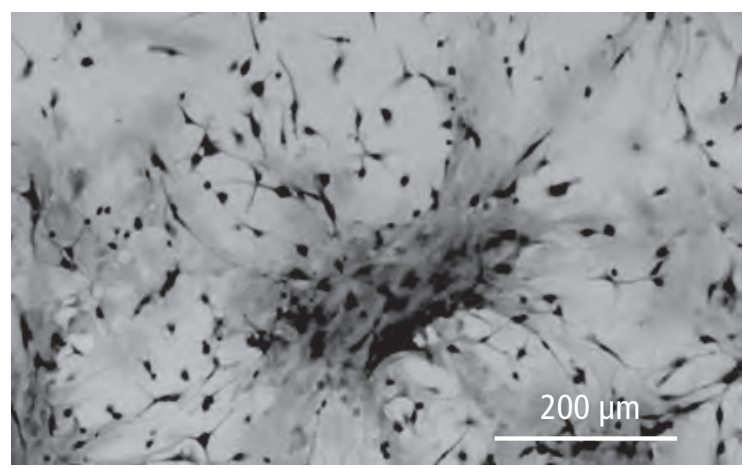

Figure 5. Alizarin-red-stained histological section of rat bone marrow cells cultured in contact with a $70: 30$ scaffold for 23 days. Fibroblast-like cells are present. No bone nodules are seen. (for full-colour figure see page 204) 

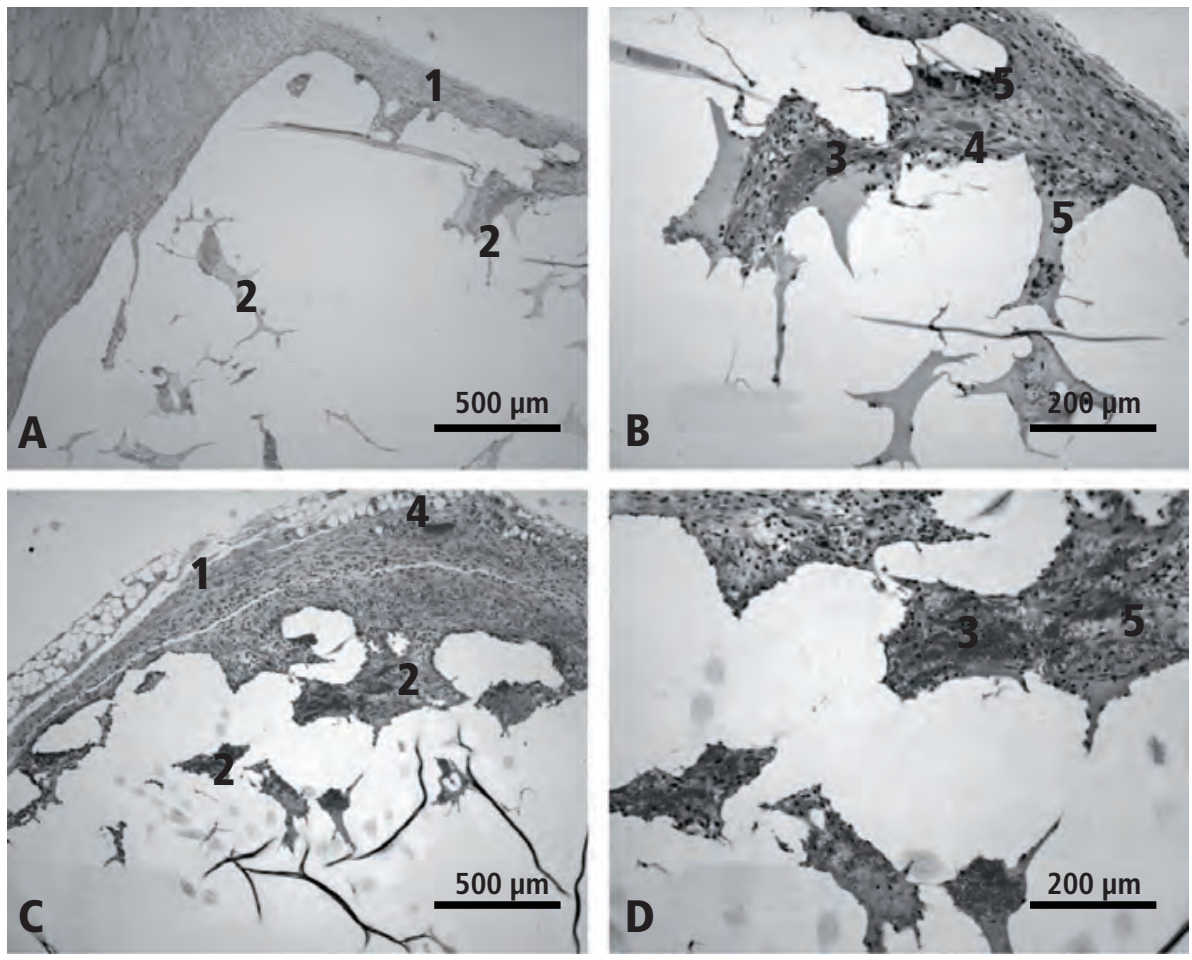

Figure 6. Haematoxylin/eosin-stained sections of specimens which were harvested 1 week after subcutaneous implantation in rats. A, B: $50: 50$ scaffold shown at different magnifications. C, D: 70 : 30 scaffold shown at different magnifications. All scaffolds are surrounded by fibrous tissue (1). Note fibroblast infiltration (2) and erythrocytes (3) in the peripheral pores of both scaffolds. Also, formation of blood capillaries (4) and some multinuclear cells (5) are seen. (for full-colour figure see page 205)

Figure 6 shows micrographs taken from samples which were harvested one week after subcutaneous scaffold implantation. The $50: 50$ scaffold group (Figures 6A and 6B) was comparable to the $70: 30$ (NVP : BMA) scaffold series (Figures $6 \mathrm{C}$ and $6 \mathrm{D}$ ). In all animals, both implants were surrounded by a thin capsule containing proliferating fibroblasts, collagen fibres, newly formed capillary sprouts and some inflammatory cells. From this capsule, endothelial cells, fibroblasts and inflammatory cells penetrated into the porous cavities at the periphery of the scaffold. Very few giant cells (multinucleated macrophages) were observed at the border of the scaffold.

A striking difference between the $50: 50$ and $70: 30$ (NVP : BMA) scaffolds was seen 84 days after subcutaneous scaffold implantation. The $50: 50$ scaffolds (Figure 7A and 7B) were invaded with endothelial cells, fibroblasts and blood 

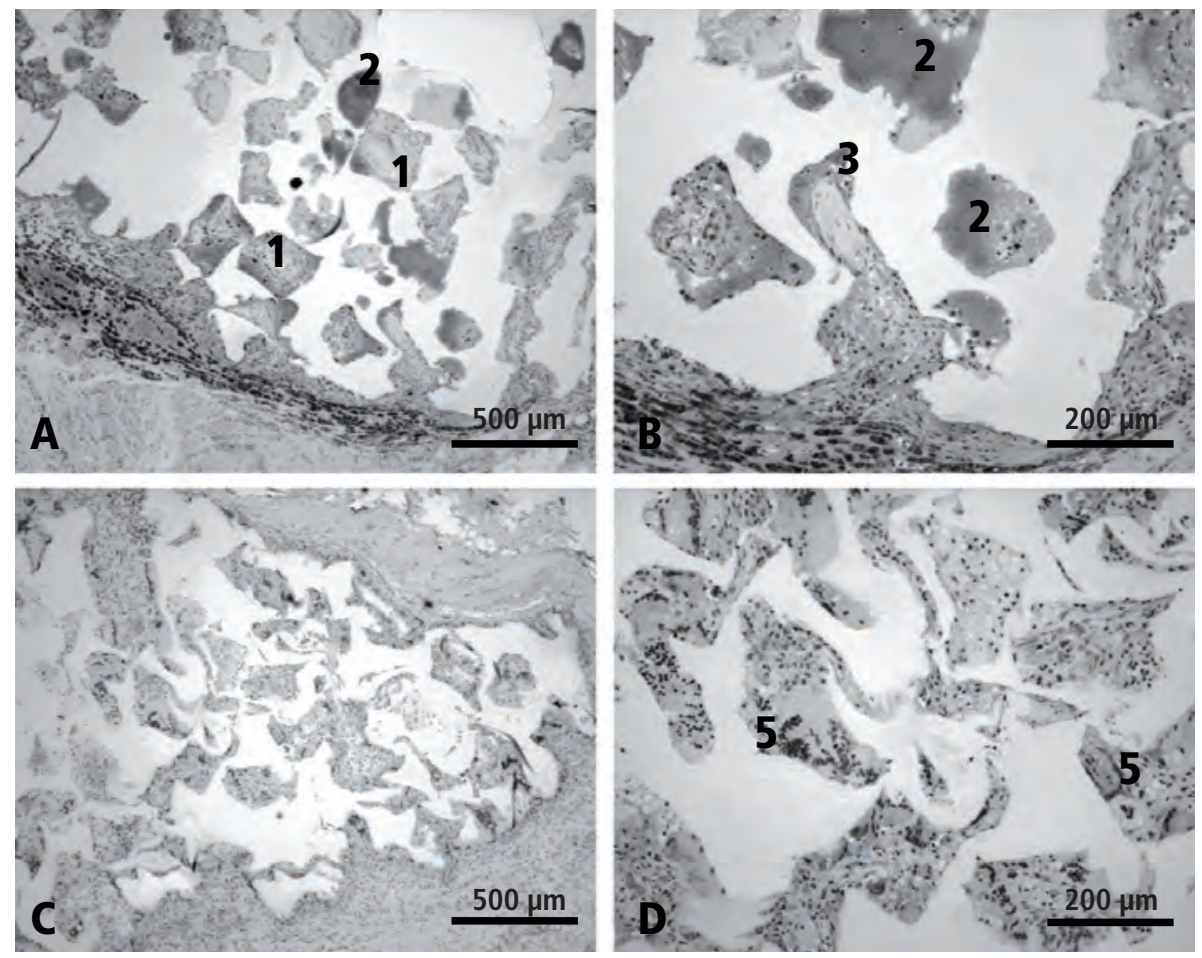

Figure 7. Haematoxylin/eosin-stained sections of specimens which were harvested 84 days after subcutaneous implantation in rats. A, B: 50 : 50 scaffolds shown at different magnifications. Note that the pores retained their rectangular shape (1). The pores are invaded by erythrocytes (2), endothelial cells and fibroblasts (3). Note the iron-containing macrophages (4) between the dermis and the 50 : 50 scaffold. C, D: 70 : 30 scaffolds shown at different magnifications. The pores in these scaffolds lost their rectangular shape, due to the softer nature of this material. The cavities are filled with fibrous tissue and many giant cells (5). (for full-colour figure see page 206)

cells. The endothelial cells formed a large cavernous vascular network filled with blood. Furthermore, a dense network was present throughout the scaffold consisting of fibroblasts, collagen and newly formed capillaries. Also, some macrophages were observed. The pores maintained their rectangular or square form, which corresponded to the dimensions of the salt crystals used in the preparation of the scaffold. The capsule around the scaffold contained many ironcontaining macrophages, which are remnants from phagocytised red blood cells. This reflected the biocompatibility of the $50: 50$ scaffolds, as well as the fact that the supply of nutrients, and the efflux of waste products from these cells was realised. Only very few giant cells were seen in the $50: 50$ group. In contrast to 
the $50: 50$ group, a severe foreign body reaction was observed in and around the $70: 30$ (NVP : BMA) scaffolds (Figures 7C and 7D). Giant cells were observed between a network of fibroblasts, collagen and newly formed capillaries. These observations revealed that only $50: 50$ scaffolds were biocompatible in vivo.

The second series of in vivo experiments were based on the work of Nimni et al. , ${ }^{360}$ and modifications made by Bahar et al. ${ }^{364}$ These workers showed that ectopic bone formation could be induced in a so-called demineralised bone matrix (DBM). Four different scaffold materials were tested in this way: $50: 50$ with $80 \%$ porosity, $50: 50$ with $90 \%$ porosity, $70: 30$ (NVP : BMA) with $80 \%$ porosity, and $70: 30$ (NVP : BMA) with $90 \%$ porosity. We then monitored the process of ectopic bone formation as closely as possible, using X-ray microradiography and histological analysis. DBMs were harvested 4, 6, or 8 weeks postimplantation.

Figure 8 shows a series of representative $\mathrm{X}$-ray micrographs. White areas

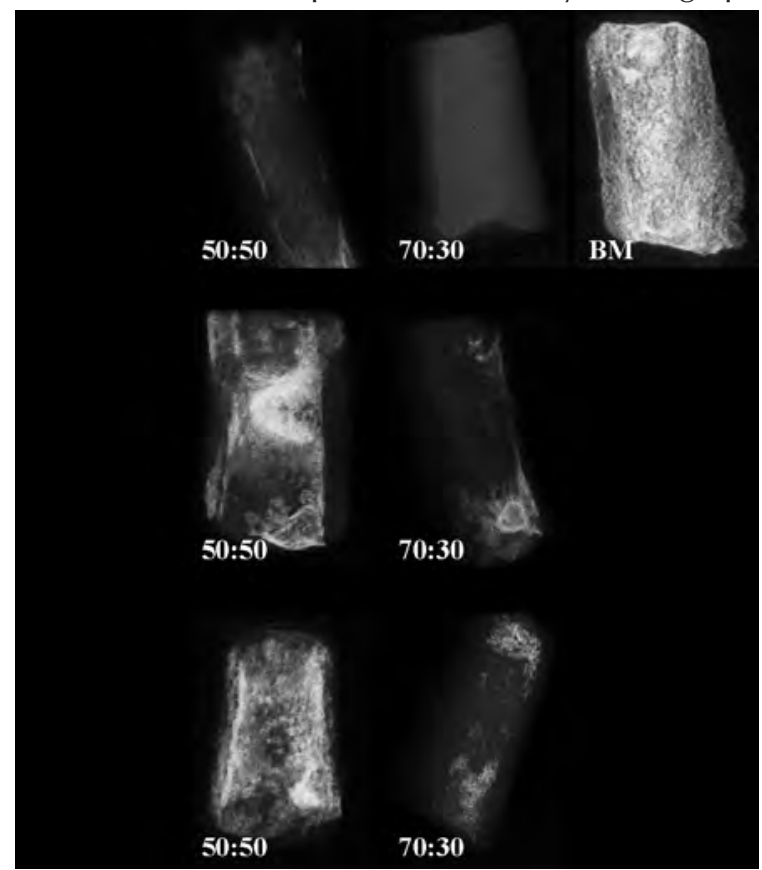

Figure 8. X-ray micrographs of explanted DBM cylinders, 4, 6 and 8 weeks post-implantation. White areas (enhanced X-ray absorption) correspond with mineralization; see Materials and Methods section fur further experimental details. DBM cylinders were filled with $50: 50$ scaffold parts and bone marrow cells (50:50); $70: 30$ scaffold parts and bone marrow cells (70:30), or with bone marrow cells alone (BM). 
(enhanced X-ray absorption) reveal mineralization. Clearly, mineralization occurred fast in the control DBM, which was entirely filled with bone marrow. In the $50: 50$ series, mineralization occurred after 6 weeks, predominantly in the centre. After 8 weeks, mineralization was seen throughout the entire volume of the DBM. In the $70: 30$ (NVP : BMA) series, there was much less mineralization. After 6 weeks and after 8 weeks post-implantation, some enhanced contrast was observed at the ends of the DBM cavity, but certainly not in the centre. We concluded from this that the 50:50 material had significantly better osteoconductivity, as compared to the $70: 30$ counterpart.

Figure 9 shows micrographs from our histological analysis of scaffolds
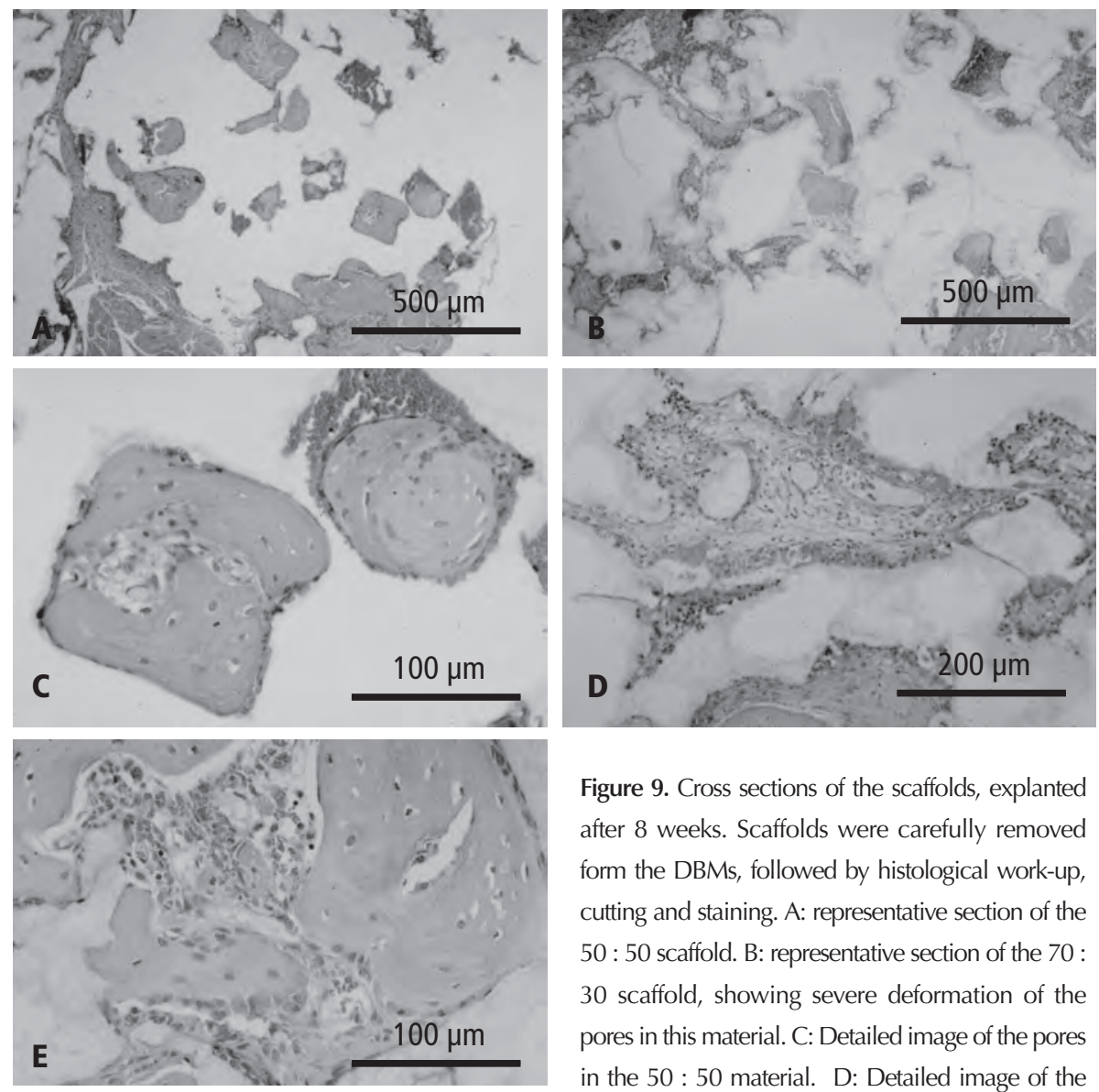

Figure 9. Cross sections of the scaffolds, explanted after 8 weeks. Scaffolds were carefully removed form the DBMs, followed by histological work-up, cutting and staining. A: representative section of the 50 : 50 scaffold. B: representative section of the 70 : 30 scaffold, showing severe deformation of the pores in this material. C: Detailed image of the pores in the 50 : 50 material. D: Detailed image of the pores in the $70: 30$ material. E: Newly formed bone in $50: 50$ scaffold. (for full-colour figure see page 207) 
which were explanted after 8 weeks, and which were subsequently removed from the DBM. Figures 9A, C and E show cross-sections of one of the $50: 50$ scaffolds; Figures $9 \mathrm{~B}$ and $\mathrm{D}$ show sections of one of the $70: 30$ (NVP : BMA) scaffolds. The pores in the in $50: 50$ scaffold were clearly visible as rectangles, which revealed that the scaffold did not deform (compare: Figure 7A,B). On the other hand, the pores in the $70: 30$ (NVP : BMA) scaffold completely lost their rectangular shape. Figure $9 \mathrm{C}$ and $9 \mathrm{E}$ provide a detailed image of the pores in the 50 : 50 material. Newly formed bone was deposited directly at the interface of the polymer with very active osteoblasts present at these sites. Figure 9D shows, an image of the pores in the $70: 30$ material (NVP : BMA); these were filled with fibrous tissue and many multinucleated giant cells.

\section{CONCLUDing REMARKS}

Tissue engineering on the basis of degradable polymeric scaffolds still poses many technical problems that are only partly understood. This study reveals that the physico-chemical properties of the scaffold biomaterials play an important role by themselves. Comparison of two stable scaffold materials, i.e. $70: 30$ (NVP : BMA) and the more hydrophobic $50: 50$ counterpart showed that the latter is less cytotoxic, more biocompatible, and more osteoconductive in vivo. A possible explanation for this difference may lie in the fact that the $50: 50$ material has a more favourable balance between hydrophobic and hydrophilic properties. Therefore the $50: 50$ material is likely to be associated with better adherence of cells, and more pronounced adsorption of proteins its surface. 
Hydrophobicity as a design criterion for polymer scaffolds 


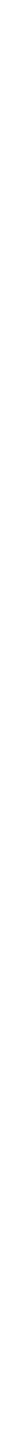




\section{CHAPTER 9 \\ General Discussion}


Chapter 9 


\section{General Discussion}

At present, multiple viscosupplementation products from various sources, with different degrees of purity, and different molecular weights, are available for medical applications. Hyaluronan is obtained from rooster crests (e.g. Hyalgan ${ }^{\circledR}$, OrthoVisc ${ }^{\circledR}$, Synvisc ${ }^{\circledR}$, Supartz ${ }^{\mathrm{TM}}$ ) or is manufactured by bacterial fermentation of Streptococci (e.g. Adant ${ }^{\mathrm{TM}}$, Biohy ${ }^{\mathrm{TM}}$, Fermathron ${ }^{\mathrm{TM}}$, Osteni $\left.{ }^{\circledR}\right)$. The bacterial production of hyaluronan by Streptococci enabled it to be produced in larger quantities than could be achieved with the extraction out of rooster combs: 4 grams of hyaluronan per liter cultivated solution versus 0.9 grams of hyaluronan per kilogram of the original material, respectively. ${ }^{369}$

The efficacy of intra-articular hyaluronan treatment in the clinical setting is controversial, which is, at least partially, due to the enormous number of reports having low methodological quality. However, the last years several randomized controlled trials (RCTs) were published. A meta-analysis of RCTs showed that hyaluronan could decrease symptoms of osteoarthritis of the knee such as improvements in pain and functional outcomes. ${ }^{70} \mathrm{~A}$ second systemic review of RCTs confirmed the therapeutic efficacy of hyaluronan treatment, but stated it has, at best, modest efficacy in the treatment of knee osteoarthritis. ${ }^{71}$ On the other hand, a third systemic review and meta-analysis on the efficacy of intra-articular hyaluronan showed no proven clinically effect on pain at rest or during exercise. Besides, hyaluronan treatment did not lead to improvement in joint function at distinct outcomes, measured repeatedly over time. ${ }^{72}$

In a systemic review using Cochrane methodology many hyaluronan and hylan derivatives of widely different molecular weights and formulation were tested. The authors concluded that treatment with hyaluronan is an effective treatment for osteoarthritis of the knee with beneficial effects on pain, function and patient global assessment. ${ }^{368}$

The mechanisms of action of hyaluronan are not fully elucidated yet. The actual period that the injected hyaluronan stays within the joint space is in the order of hours to days, but the time of clinical efficacy is often in the order of months. Therefore other mechanisms than lubricating the joint have been postulated to explain the prolonged action. These include anti-inflammatory and antinociceptive properties or stimulation of in vivo synthesis.

The results of our rabbit study confirm the beneficial effects of hyaluronan in the knee. Hyaluronan has a potential role in preventing cell death following articular cartilage injury, and improves cartilage metabolism in knees with 6- 
month-old cartilage defects. This might explain the reduction in pain and joint effusion together with an improvement in daily activities when hyaluronan was injected at the end of an arthroscopic partial meniscectomy. ${ }^{196}$

The best indication for intraarticular treatment with hyaluronan has yet to be defined with respect to age and level of osteoarthritis as defined radiographically. It seems that patients over 65 years of age and those with complete loss of joint space on radiographs are less likely to benefit from hyaluronan therapy. ${ }^{70}$ Also, optimal dosing regimens have to be developed, and the most appropriate positioning in treatment algorithms have to be determined.

Compared with lower-molecular-weight hyaluronan, the highest-molecularweight formulation may have greater effects. ${ }^{370}$ Recently, it was shown in a prospective randomized clinical trial that Hylan G-F $20^{\circledR}$ (cross-linked derivatives of hyaluronan; MW, 6 million Daltons) was more effective than Hyalgan ${ }^{\circledR}$ (fraction of purified natural sodium hyaluronan; MW, $0.50-0.73$ million Daltons) in patients with osteoarthritis of the knee. ${ }^{371}$ Pain reduction occurred earlier and lasted for much longer in the Hylan G-F $20^{\circledR}$ as compared to the Hyalgan group. However, incidence of adverse events was higher in the Hylan G-F $20^{\circledR}$ group.

Most case series of results of cartilage repair techniques lack methodological quality, which indicates that caution is required when interpreting results after surgical cartilage repair. ${ }^{372,} 373$ However, few RCTs were published in the last years:

(1) $\mathrm{ACl}$ and mosaicplasty were compared in two RCTs. In the first study, ${ }^{374}$ both therapies resulted in a decrease in symptoms, while recovery after $\mathrm{ACl}$ was slower than that after osteochondral transplantation. Histologically, the defects treated with $\mathrm{ACl}$ were primarily filled with fibrocartilage, whereas the osteochondral cylinder transplants retained their hyaline character. However, a persistent interface between the transplant and the surrounding original cartilage was observed. In the second study $88 \%$ had excellent or good results after $\mathrm{ACl}$ compared with $69 \%$ after mosaicplasty. ${ }^{375}$ Arthroscopy at one year demonstrated excellent or good repairs in $82 \%$ after $\mathrm{ACl}$ and in $34 \%$ after mosaicplasty.

(2) Microfracture and $\mathrm{ACl}$ were compared with a follow-up period of 2 and 5 years. ${ }^{376,} 377$ Both groups had significant clinical improvement with 
satisfactory results in $77 \%$ of the patients at 5 years. Subanalyses of the 2 year follow-up results demonstrated that treatment of smaller lesions with microfracture yielded better clinical outcomes than did treatment of larger lesions, an effect not observed in $\mathrm{ACl}$ group. ${ }^{378}$ One-third of the patients had early radiographic signs of osteoarthritis 5 years after the surgery without significant difference in the clinical and radiographic results between the two treatment groups. Besides, no correlation between the histological findings and the clinical outcome was observed.

(3) Recently, the efficacy of characterized chondrocyte implantation (CCl) versus microfracture was compared in patients having a single cartilage lesion of the femoral condyle. ${ }^{379} \mathrm{CCl}$ resulted in better structural repair than microfracture as assessed by histomorphometry. Evaluation of overall histological components showed more chondrocyte-like cells and a higher proteoglycan content of the cellular matrix. Clinical outcome, however, was comparable at 12 and 18 months after treatment.

A comparative study of microfracture and drilling in rabbits was presented at the $54^{\text {th }}$ Annual Meeting of the Orthopaedic Research Society. ${ }^{380}$ The study revealed that significant differences were found in acute subchondral damage and subsequent repair responses. Microfracture induced acute fracturing and crushing of bone resulting in compacted bone surrounding the holes that could impede repair and access to cancellous marrow.

Patient selection, size and location of the defect, and the postoperative rehabilitation regime cast doubt about the validity of the comparison of the different cartilage repair strategies. Besides, studies have to be adequately powered to demonstrate a difference between groups. Additional good quality RCTs with long-term functional outcomes are required to compare the results of existing treatments.

As mentioned in chapter 1 major limitations of $\mathrm{ACl}$ are periosteal hypertrophy, detachment of the periosteal flap, transplant failure, and cartilage damage due to suturing the periosteal flap onto the surrounding cartilage. The harvesting of periosteum also increases the operating time and requires a larger surgical exposure, which may be associated with increased pain and arthrofibrosis. Besides, the implantation of cultured chondrocytes in suspension has led to concerns about the uneven distribution of chondrocytes within the defect and the potential for cell leakage. ${ }^{381}$

Limitations with mosaicplasty are the amount of grafts available, donor 
site morbidity, difficulty to match the topology of the graft with the injured site, and failure of integration with the surrounding tissue.

Therefore, research has been focused on repair of articular surfaces by tissue engineering applications using scaffolds, because it has the potential to overcome these limits. The last years have seen continuous refinement and improvement of tissue engineering strategies using polymer-based scaffolds. An understanding of the requirements to restore a normal joint function is still poor. For example, the optimal biomaterial for cartilage tissue engineering purposes has to be determined regarding its biomechanical and biochemical properties.

It is a generally accepted thesis that in tissue engineering of load-bearing tissues the scaffold initially should have similar mechanical properties as the tissue to be regenerated. However, in this thesis we showed that PEOT/PBT based scaffolds with low mechanical properties were superior in cartilage repair tissue formation. Unfortunately, due to these properties the more hydrophilic scaffold lost its stiffness after pre-wetting, which made implantation of the scaffold troublesome. With the objective of using PEOT/PBT scaffolds in cartilage tissue engineering applications in the human setting, it has to be investigated whether decreasing the hydrophilicity will improve scaffold handling without loosing its cartilage stimulating properties.

Furthermore, the feasibility of using biphasic or bilayered scaffolds for osteochondral repair is indicated. A bilayered scaffold comprised of a top layer of soft and hydrophilic PEOT/BPT 70/30 to stimulate chondrogenesis, and a bottom layer of stiff and hydrophobic PEOT/PBT 55/45 to support subchondral bone formation may have added value for osteochondral tissue engineering. In addition, the biphasic shell-core 3D deposited scaffolds fabricated out of fibres comprised of a core of PEOT/PBT 55/45 and a shell of PEOT/PBT 70/30 are also of interest.

Also, the in vivo behaviour of PEOT/PBT 55/45 scaffolds seeded with allogeneic or autogenic chondrocytes is the subject of further experiments. Besides, long-term investigations need to be undertaken to confirm the longevity of the repair tissue.

Next to the in vivo studies with PEOT/PBT scaffolds we investigated the non-absorbable copolymer NVP/BMA. These series of experiments showed that the more hydrophobic scaffold was more biocompatible than the hydrophilic counterpart, which, again, showed that the physico-chemical properties of the scaffold biomaterials play an important role by themselves.

Promising novel approaches are scaffolds fabricated using rapidprototyping techniques with a computer-aided design; ${ }^{159}$ shape memory materials, $^{382}$ like alginate hydrogels, ${ }^{383}$ or pastes seeded with cells that can be 
introduced arthroscopically in a condensed state and acquire their shape in situ. One method to overcome the limitation of donor tissue and donor site morbidity is to use extra-articular cells. We showed that periosteum, harvested from the proximal tibia of elderly patients, can be used as alternative cell source. Despite the decreased chondrogenic potential, periosteum-derived cells could be expanded and redifferentiated to the chondrogenic lineage. However, it took about 3 months to obtain sufficient cells (sufficient for seeding into a porous scaffold), which is way too long for clinical practice purposes. Addition of growthfactors accelerated expansion of periosteum-derived cells. Optimizing the harvesting technique (using a periosteal elevator), use of a selective cell culture medium, in combination with the recently developed cell sorting procedure, may significantly increase the potential of periosteum as a source for chondrogenic cells.

Next to the use of mesenchymal stem cells as alternative cell source for chondrocytes in scaffold tissue engineering, novel improvements in cell biology have been described recently.

First, Hendriks et al. showed that non-expanded chondrocytes can induce chondrogenic differentiation of other cell types, and emphasized the role of the cell - cell communication. ${ }^{384}$

Secondly, Emans et al. showed that due to manipulation of the layer between the periosteum and bone a cartilage-like tissue can be formed. ${ }^{385}$ This extra-articular source of cartilage may be useful in the repair of an articular cartilage lesion. Using this reactive tissue bypasses the problems accompanying cell proliferation and can be harvested without damaging the articular cartilage. Investigations are currently going on to determine the optimal technique of inducing periosteal chondrogenesis.

Also scaffolds delivered without requiring ex vivo cell expansion is of interest. ${ }^{386}$ Cartilage tissue is minced into small fragments and loaded onto the scaffold. The fragments are retained with a coating of fibrin clot to form a construct for culture. Treatment of chondral defects in goats using resorbable scaffolds loaded with cartilage fragments produced hyaline-like repair tissue at 6 months. 



\section{CHAPTER 10}

Summary

Nederlandse Samenvatting 
Chapter 10 
In Chapter 1 the incidence, types, and natural course of articular cartilage lesions are discussed. Subsequently, structure and function of articular cartilage is described. Also, an overview of currently applied conservative and surgical interventions is given.

In Chapter 2 the aims of this thesis are explained, which all are aimed to further optimize tools to repair cartilage defects and prevent osteoarthritis.

In Chapter 3 it was shown that partial-thickness articular cartilage lesions in the rabbit knee did not heal, and resulted in changes suggesting early degeneration.

We used a rabbit-model that better reflects the clinical situation, including an extended period of preoperative cartilage damage, and would be better suited for evaluating experimental cartilage repair techniques in the future.

Using this model we evaluated the effect of a partial-thickness articular cartilage lesion on the surrounding cartilage with regard to macroscopic, microscopic, and biochemical parameters during the course of 26 weeks. A circumscribed 4-mm-diameter partial-thickness cartilage defect was created on one medial femoral condyle without concomitant injuries of the meniscus or anterior cruciate ligament, while the contralateral knee was sham-treated.

In experimental knees, we found cartilage softening and fibrillation at 13 and 26 weeks. Degenerative changes observed at 1 week were partially restored at 13 weeks but worsened later and were most pronounced at 26 weeks. Histologic scores in the experimental groups were worse at 1 and 26 weeks when compared with the sham groups. Degenerative changes at 26 weeks improved compared with 1 week in the sham groups. Disturbances in proteoglycan metabolism were less evident.

The used rabbit model has advantages compared with other animal models:

(1) when similar diameters are used, the effect of cartilage repair techniques can be monitored without the confounding effects of other potential causes of cartilage degeneration;

(2) the operation is relatively simple and creates circumscribed cartilage lesions;

(3) repair of these chronic partial-thickness articular cartilage lesions occurs with surrounding degeneration, which resembles the clinical situation of a focal cartilage lesion; and

(4) cartilage lesions are created on the medial femoral condyle, which is the most commonly affected zone of articular cartilage damage observed with arthroscopies in humans. 
In Chapter 4 was revealed that hyaluronan has a chondroprotective effect on the short-term when applied immediately post-injury, and improves chondrocyte metabolism in knee joints with long-existing lesions.

First we examined the effect of hyaluronan on articular cartilage surrounding iatrogenic cartilage lesions, which can occur during arthroscopic procedures. Rabbits knees were rinsed with $0.9 \% \mathrm{NaCl}$ after creating partialthickness articular cartilage lesions. Experimental knees were treated immediately with hyaluronan; contralateral knees received $0.9 \% \mathrm{NaCl}$. Rabbits were sacrificed at 2 days or 3 months postoperatively. Using histomorphometric analysis it was shown that treatment with hyaluronan resulted in the protection of chondrocytes peripheral to the cartilage defect, whereas a relatively high percentage of dead cells was observed in untreated knees 2 days after creating the defect. It remained unclear as to whether hyaluronan is chondroprotective in the long-term: The percentage of dead chondrocytes in hyaluronan-treated knees did not increase between 2 days and 13 weeks postoperatively. On the other hand, in untreated knees the percentage of dead chondrocytes decreased, showing comparable percentages of cell death as those seen in the hyaluronantreated knees at 3 months follow-up.

In the second series of experiments we showed that also in knee joints with long-existing lesions hyaluronan can restore the impaired chondrocyte metabolism, caused by the irrigation procedure. Rabbit knee joints having 6month-old partial-thickness articular cartilage defects were used. Knee joints i) were not irrigated; or ii) were irrigated with $0.9 \% \mathrm{NaCl}$; or iii) received hyaluronan after irrigation with $0.9 \% \mathrm{NaCl}$. Seven days postoperatively, patellae were harvested to study the total sulphate incorporation rate. Hyaluronan treatment resulted in significantly higher sulphate incorporation compared to knees irrigated with $0.9 \% \mathrm{NaCl}$.

In Chapter 5 it was shown that PEOT/PBT 70/30 scaffolds, having low mechanical strength, performed better in the healing of osteochondral defects than the PEOT/PBT 55/45 scaffolds that most closely match the biomechanical properties of native articular cartilage.

We compared the in vivo healing response of osteochondral defects in medial femoral condyles in rabbits that were filled with a cell-free PEOT/PBT 70/30 scaffold or the stiffer PEOT/PBT 55/45 scaffold.

Repair tissue in PEOT/PBT 70/30 scaffolds consisted of cartilage-like tissue on top of trabecular bone, whereas the tissue within the PEOT/PBT 55/45 scaffolds consisted predominantly of trabecular bone. The histological cartilage repair score of lesions treated with PEOT/PBT 70/30 scaffolds was significantly 
better compared to those of untreated osteochondral defects or lesions treated with PEOT/PBT 55/45 scaffolds.

In Chapter $\mathbf{6}$ we showed that cells isolated from periosteum from elderly humans have chondrogenic potential.

By expanding cells in minimum essential medium D-valine, the selection of progenitor cells was favoured, which resulted in a higher cartilage yield for tissue engineering applications. This selective medium has been used in a variety of cell cultures, but thus far it was not known whether mesenchymal stem cells could proliferate in minimum essential medium D-valine.

The use of fetal bovine serum proved to be essential for the proliferation of periosteum-derived cells. The addition of FGF-2, IGF-1 and neAA to serumcontaining medium increased the proliferation rate. Differentiation towards a chondrocyte phenotype was achieved in representative high-density cell culture systems with addition of ascorbic acid-2-phosphate, IGF-1, TGF $\beta_{1}$, $\mathrm{TGF}_{2}$, and/or TGF $\beta_{3}$. Regardless of the TGFß isomer used, type II collagen mRNA was expressed in $59 \%$ of the samples to which exogenous TGFß was added.

In Chapter 7 it was revealed that the infection rates of cell-free and cellseeded PEOT/PBT based scaffolds are as significant a problem as those encountered in traditional biomaterials-based implants.

Over the past 5 years we have performed several preclinical animal studies to evaluate degradable polyester scaffolds for tissue engineering of articulating joint surfaces. Over this time, a total of 228 polymer scaffolds have been implanted into standard osteochondral defects in rabbit knees for periods of 3 weeks up to 9 months. Scaffolds were prepared by compression moulding or by rapid prototyping, and sterilized by $\gamma$-irradiation. Furthermore, scaffolds of a single copolymer composition, and blended scaffolds were used. Of these, devices were implanted without cells or were seeded with allogeneic or autologous chondrocytes and then surgically implanted. Infections were seen across all scaffold types, regardless of polymer compositions, blended formulations, or available pore volume in compression moulded and printed scaffolds.

In Chapter 8 we revealed that the physico-chemical properties of the scaffold biomaterials play an important role by themselves.

We report the results of a series of in vitro and in vivo experiments using stable NVP/BMA (1-vinyl-pyrrollidinone/n-butyl-methacrylate) based scaffolds. 
Biocompatibility in vitro was studied by MTT assay using mouse fibroblasts and a direct contact method using isolated rat calvarial cells. Biocompatibility in vivo was studied by implantation scaffolds subcutaneously in rats and scaffold participation in the demineralised bone model. These experiments showed that the hydrophobic NVP/BMA devices are less cytotoxic, more biocompatible, and more osteoconductive in vivo. 


\section{Nederlandse samenvatting van de conclusie}

In Hoofdstuk 1 worden de incidentie, verschillende typen, en natuurlijk beloop van gewrichtskraakbeen letsels besproken. Vervolgens worden structuur en functie van gewrichtskraakbeen beschreven. Ook wordt een overzicht gegeven van de huidig toegepaste conservatieve en operatieve interventies.

In Hoofdstuk 2 worden de doelen van dit proefschrift vermeld, welke zijn gericht op het verder optimaliseren van technieken die herstel van kraakbeen defecten en preventie van artrose beogen.

In Hoofdstuk 3 lieten we zien dat "partial-thickness" kraakbeen defecten in konijnenknieën niet herstelden, en leidden tot vroeg degeneratieve veranderingen.

Wij gebruikten een konijnenmodel dat de klinische situatie meer nabootste, met een lang tijdsinterval tussen ontstaan van het defect en operatief ingrijpen, welke in de toekomst beter past in het evalueren van experimentele kraakbeen herstel technieken.

Wij evalueerden het effect van een "partial-thickness" gewrichtskraakbeen defect op het omringende kraakbeen gedurende 26 weken, gebruik makend van macroscopische, microscopische en biochemische parameters. Op een mediale femurcondyl werd een "partial-thickness" kraakbeen defect gemaakt met een diameter van 4 millimeter zonder begeleidend letsel van de meniscus of voorste kruisband, terwijl de contralaterale knie een "sham" ingreep kreeg.

Het kraakbeen van de experimentele knieën was na 13 en 26 weken zacht en toonde fibrillaties. De degeneratieve veranderingen die na 1 week werden gezien, waren na 13 weken deels hersteld, maar verergerden en waren meest prominent aanwezig na 26 weken. $\mathrm{Na} 1$ en 26 weken waren de histologische scores in de experimentele groepen slechter dan in de "sham" groepen. Degeneratieve veranderingen die in de sham groepen na 1 week werden gezien, waren verbeterd na 26 weken.

Stoornissen in het proteoglycanen metabolisme waren minder evident.

Het gebruikte konijnenmodel heeft voordelen vergeleken met andere diermodellen:

(1) bij gelijke diameters kan het effect van kraakbeentechnieken bestudeerd worden zonder vertroebelende effecten van andere potentiële oorzaken van kraakbeen degeneratie;

(2) de operatie is relatief gemakkelijk en resulteert in goed omschreven kraakbeenlaesies; 
(3) herstel van deze chronische "partial-thickness" gewrichtskraakbeen defecten gaat samen met degeneratie van het omliggende kraakbeen, overeenkomstig met de klinische situatie van een focaal kraakbeendefect; en

(4) kraakbeen defecten worden op de mediale femurcondyl gemaakt, welke tijdens artroscopieën bij mensen de meest voorkomende aangedane locatie is van een kraakbeendefect.

In Hoofdstuk 4 werd getoond dat hyaluronzuur op de korte termijn een beschermend effect op kraakbeen heeft als het direct na het trauma wordt toegediend, en dat in knieën met langerbestaande defecten het metabolisme van chondrocyten verbetert.

Ten eerste onderzochten wij het effect van hyaluronzuur op gewrichtskraakbeen rondom een iatrogeen kraakbeen defect, zoals tijdens een artroscopie kan gebeuren. In konijnenknieën werd een "partial-thickness" gewrichtskraakbeen defect gemaakt, waarna deze werden gespoeld met $0.9 \%$ $\mathrm{NaCl}$. Vervolgens werden de experimentele knieën direct behandeld met hyaluronzuur; de contralaterale knieën kregen $0.9 \% \mathrm{NaCl}$ toegediend. De konijnen werden 2 dagen of 3 maanden postoperatief opgeofferd. Na 2 dagen werd door middel van histomorphometrische analyse aangetoond dat in de hyaluronzuur behandelde groep een beduidend lager percentage dode cellen aanwezig was in vergelijking met de controlegroep. Het blijft echter onduidelijk of hyaluronzuur effectief is op de lange termijn: In de hyaluronzuurbehandelde knieën nam het percentage dode cellen niet toe. Daarentegen nam het percentage dode cellen in de onbehandelde knieën af. Na drie maanden was het percentage dode cellen in beide groepen vrijwel gelijk.

In de tweede studie lieten we zien dat hyaluronzuur het verstoorde chondrocyt metabolisme, als gevolg van het spoelen, ook in kniegewrichten met langerbestaande defecten kan herstellen.

Konijnenkniegewrichten met 6-maanden-oude "partial-thickness" gewrichtskraakbeenletsels werden gebruikt. Kniegewrichten i) werden niet gespoeld; of ii) werden gespoeld met $0.9 \% \mathrm{NaCl}$; of iii) kregen hyaluronzuur toegediend na het spoelen met $0.9 \% \mathrm{NaCl}$. Na 7 dagen werden de knieschijven geoogst om de totale sulfaatinbouw te bestuderen. Behandeling met hyaluronzuur resulteerde in een significant hogere sulfaat inbouw in vergelijking met knieën die werden gespoeld met $0.9 \% \mathrm{NaCl}$.

In Hoofdstuk 5 lieten we zien dat osteochondraal defecten die behandeld waren met 70/30 scaffolds (PEOT/PBT), gekenmerkt door lage mechanische 
eigenschappen, beter herstelden dan osteochondraal defecten die met 55/45 scaffolds (PEOT/PBT), waren behandeld. De laatste benadert de biomechanische eigenschappen van kraakbeen.

We vergeleken het in vivo herstel van osteochondraal defecten in mediale femurcondylen van konijnen die met een celvrije 70/30 scaffold of de stijvere 55/45 scaffold werden gevuld. Het herstel weefsel in 70/30 behandelde defecten bestond uit kraakbeenachtig weefsel waaronder trabeculair bot, terwijl het weefsel in de met 55/45 scaffold behandelde defecten grotendeels uit trabeculair bot bestond. De histologische score voor kraakbeenherstel van defecten die met 70/30 scaffolds waren behandeld was significant beter dan die van onbehandelde defecten of defecten behandeld met 55/45 scaffolds.

In Hoofdstuk 6 toonden wij aan dat periosteum van ouderen chondrogeen potentiaal heeft.

De selectie van mesenchymale stamcellen kan verbeterd worden door cellen te expanderen in "minimum essential medium D-valine". Dit resulteert in een hogere kraakbeenopbrengst voor tissue engineering doeleinden. "Minimum essential medium D-valine" is in verscheidene celkweken gebruikt, maar tot dusver was het onbekend of mesenchymale cellen hierin konden prolifereren.

Het gebruik van "fetal bovine serum" bleek essentieel te zijn voor de proliferatie van cellen die uit periosteum verkregen waren. De toevoeging van FGF-2, IGF-1 en neAA aan serumrijk medium verhoogde de proliferatie snelheid.

Differentiatie naar een chondrocyt fenotype werd bereikt in representatieve "high-density cell culture" systemen met toevoeging van vitamine C, IGF-1, $\mathrm{TGF}_{1}, \mathrm{TGF}_{2}$, en/of TGF $\beta_{3}$. Ongeacht de gebruikte TGFß isomeer, werd in 59\% van de monsters, waaraan TGFß was toegevoegd, expressie van type II collageen mRNA gezien.

In Hoofdstuk 7 werd beschreven dat het infectie percentage van celvrije en celhoudende PEOT/PBT scaffolds een even groot probleem is als die van de hedendaags gebruikte implantaten.

In de laatste 5 jaren werden meerdere preklinische dierstudies verricht om degradeerbare polyester scaffolds voor tissue engineering van gewrichtoppervlakken te bestuderen. Gedurende deze periode werden 228 scaffolds in osteochondraal defecten van konijnenknieën geïmplanteerd met een follow-up van 3 weken tot 9 maanden. Scaffolds werden geproduceerd middels "compression moulding" danwel door "rapid prototyping", en gesteriliseerd 
door $\gamma$-straling. Daarbij werden scaffolds gebruikt bestaande uit 1 co-polymeer en scaffolds gevormd uit meerdere co-polymeren. Scaffolds zonder cellen, en scaffolds met allogene of autogene chondrocyten werden geïmplanteerd. Infecties werden bij alle soorten scaffolds gezien, ongeacht de samenstelling van de scaffold, gemengde samenstellingen, of porievolume in "compression moulded" en "printed" scaffolds.

In Hoofdstuk 8 werd aangetoond dat de fysisch-chemische eigenschappen van scaffold biomaterialen op zichzelf een belangrijke rol spelen.

Wij beschreven de resultaten van in vitro en in vivo experimenten met scaffolds van een niet-degradeerbaar biomateriaal, NVP/BMA (1-vinyl-pyrrollidinone/n-butylmethacrylaat). Biocompatibiliteit in vitro werd bestudeerd middels een MTT test gebruik makend van muis fibroblasten, en een "direct contact" methode met geïsoleerde cellen van rattenschedels. Biocompatibiliteit in vivo werd bestudeerd door middel van het subcutaan implanteren van scaffolds in ratten en de participatie van scaffolds in het gedemineraliseerde botmodel. Deze experimenten toonden aan dat hydrofobe NVP/BMA scaffolds minder cytotoxisch; biocompatibeler; en osteoconductiever in vivo blijken te zijn. 


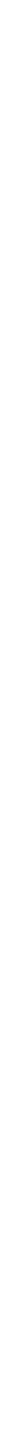


References 


\section{REFERENCES}

1. Aroen A, Loken S, Heir S, et al. 2004. Articular cartilage lesions in 993 consecutive knee arthroscopies. Am J Sports Med 32:211-215.

2. Brittberg M, Lindahl A, Nilsson A, et al. 1994. Treatment of deep cartilage defects in the knee with autologous chondrocyte transplantation. New England Journal of Medicine 331:889-895.

3. Curl WW, Krome J, Gordon ES, et al. 1997. Cartilage Injuries: A review of 31,516 Knee Arthroscopies. Arthroscopy 13:456-460.

4. Hardaker WT, Jr., Garrett WE, Jr., Bassett FH, 3rd. 1990. Evaluation of acute traumatic hemarthrosis of the knee joint. South Med J 83:640-644.

5. Noyes FR, Bassett RW, Grood ES, et al. 1980. Arthroscopy in acute traumatic hemarthrosis of the knee. Incidence of anterior cruciate tears and other injuries. J Bone Joint Surg Am 62:687-695, 757.

6. Widuchowski W, Widuchowski J, Trzaska T. 2007. Articular cartilage defects: study of 25,124 knee arthroscopies. Knee 14:177-182.

7. Hjelle K, Solheim E, Strand T, et al. 2002. Articular cartilage defects in 1,000 knee arthroscopies. Arthroscopy 18:730-734.

8. O'Driscoll SW. 2001. Preclinical cartilage repair: current status and future perspectives. Clin Orthop 391S:S397-401.

9. Reinholz GG, Lu L, Saris DB, et al. 2004. Animal models for cartilage reconstruction. Biomaterials 25:1511-1521.

10. Shapiro F, Koide S, Glimcher MJ. 1993. Cell origin and differentiation in the repair of full-thickness defects of articular cartilage. Journal of Bone and Joint Surgery. American 75-A:532-553.

11. Furukawa T, Eyre DR, Koide S, et al. 1980. Biochemical studies on repair cartilage resurfacing experimental defects in the rabbit knee. Journal of Bone and Joint Surgery. American 62-A:79-89.

12. Messner K, Gillquist J. 1996. Cartilage repair. A critical review. Acta Orthop Scand 67:523-529.

13. Wei X, Gao J, Messner K. 1997. Maturation-dependent repair of untreated osteochondral defects in the rabbit knee joint. J Biomed Mater Res 34:63-72.

14. Colwell CW, Jr., D'Lima DD, Hoenecke HR, et al. 2001. In vivo changes after mechanical injury. Clin Orthop Relat Res S116-123.

15. Brown TD, Pope DF, Hale JE, et al. 1991. Effects of Osteochondral Defect Size on Cartilage Contact Stress. Journal of Orthopaedic Research 9:559-567.

16. Guettler JH, Demetropoulos CK, Yang KH, et al. 2004. Osteochondral defects in the human knee: influence of defect size on cartilage rim stress and load redistribution to surrounding cartilage. Am J Sports Med 32:1451-1458. 
17. Costouros JG, Kim HT. 2007. Preventing chondrocyte programmed cell death caused by iatrogenic injury. Knee 14:107-111.

18. Chen CT, Burton-Wurster N, Borden C, et al. 2001. Chondrocyte necrosis and apoptosis in impact damaged articular cartilage. J Orthop Res 19:703-711.

19. Redman SN, Dowthwaite GP, Thomson BM, et al. 2004. The cellular responses of articular cartilage to sharp and blunt trauma. Osteoarthritis Cartilage 12:106-116.

20. Tew SR, Kwan AP, Hann A, et al. 2000. The reactions of articular cartilage to experimental wounding: role of apoptosis. Arthritis Rheum 43:215-225.

21. Martin JA, Brown T, Heiner A, et al. 2004. Post-traumatic osteoarthritis: the role of accelerated chondrocyte senescence. Biorheology 41:479-491.

22. Dai SM, Shan ZZ, Nakamura H, et al. 2006. Catabolic stress induces features of chondrocyte senescence through overexpression of caveolin 1: possible involvement of caveolin 1-induced down-regulation of articular chondrocytes in the pathogenesis of osteoarthritis. Arthritis Rheum 54:818-831.

23. Martin JA, Buckwalter JA. 2003. The role of chondrocyte senescence in the pathogenesis of osteoarthritis and in limiting cartilage repair. J Bone Joint Surg Am 85A Suppl 2:106-110.

24. D'Lima DD, Hashimoto S, Chen PC, et al. 2001. Human chondrocyte apoptosis in response to mechanical injury. Osteoarthritis Cartilage 9:712-719.

25. Saris DB, Dhert WJ, Verbout AJ. 2003. Joint homeostasis. The discrepancy between old and fresh defects in cartilage repair. J Bone Joint Surg Br 85:1067-1076.

26. Rodrigo JJ, Steadman JR, Syftestad G, et al. 1995. Effects of human knee synovial fluid on chondrogenesis in vitro. Am J Knee Surg 8:124-129.

27. Hogervorst T, Pels Rijcken TH, Rucker D, et al. 2002. Changes in bone scans after anterior cruciate ligament reconstruction: a prospective study. Am J Sports Med 30:823-833.

28. Buckwalter JA, Mankin HJ. 1998. Articular cartilage: degeneration and osteoarthritis, repair, regeneration, and transplantation. Instr Course Lect 47:487-504.

29. Marijnissen AC, van Roermund PM, Verzijl N, et al. 2002. Steady progression of osteoarthritic features in the canine groove model. Osteoarthritis Cartilage 10:282-289.

30. McCauley TR, Kornaat PR, Jee WH. 2001. Central osteophytes in the knee: prevalence and association with cartilage defects on MR imaging. AJR Am J Roentgenol 176:359364.

31. Tanaka S, Hamanishi C, Kikuchi H, et al. 1998. Factors related to degradation of articular cartilage in osteoarthritis: A review. Seminars of Arthritis and Rheumatism 27:392-399.

32. Linden B. 1977. Osteochondritis dissecans of the femoral condyles: a long-term followup study. J Bone Joint Surg Am 59:769-776.

33. Hunziker EB. 2002. Articular cartilage repair: basic science and clinical progress. A review of the current status and prospects. Osteoarthritis Cartilage 10:432-463. 
34. Gilbert JE. 1998. Current treatment options for the restoration of articular cartilage. American Journal of Knee Surgery 11:42-46.

35. Twyman RS, Desai K, Aichroth PM. 1991. Osteochondritis dissecans of the knee. A long-term study. J Bone Joint Surg Br 73:461-464.

36. Mankin HJ. 1974. The reaction of articular cartilage to injury and osteoarthritis (first of two parts). N Engl J Med 291:1285-1292.

37. Mankin HJ. 1982. The response of articular cartilage to mechanical injury. Journal of Bone and Joint Surgery. American 64-A:460-468.

38. Buckwalter JA, Mankin HJ. 1998. Articular cartilage: tissue design and chondrocytematrix interactions. Instr Course Lect 47:477-486.

39. Maroudas A, Bayliss MT, Venn MF. 1980. Further studies on the composition of human femoral head cartilage. Ann Rheum Dis 39:514-523.

40. Hunziker EB. 1999. Biologic repair of articular cartilage. Defect models in experimental animals and matrix requirements. Clin Orthop Relat Res S135-146.

41. Archer CW, Francis-West P. 2003. The chondrocyte. Int J Biochem Cell Biol 35:401-404.

42. Kuettner KE. 1992. Biochemistry of articular cartilage in health and disease. Clin Biochem 25:155-163.

43. Eyre DR. 1991. The collagens of articular cartilage. Semin Arthritis Rheum 21:2-11.

44. Hardingham TE, Fosang AJ. 1995. The structure of aggrecan and its turnover in cartilage. J Rheumatol Suppl 43:86-90.

45. Fosang AJ, Hardingham TE. 1989. Isolation of the N-Terminal Globular Protein Domains from Cartilage Proteoglycans - Identification of G2 Domain and Its Lack of Interaction with Hyaluronate and Link Protein. Biochemical Journal 261:801-809.

46. Vasios G, Nishimura I, Konomi H, et al. 1988. Cartilage type IX collagen-proteoglycan contains a large amino-terminal globular domain encoded by multiple exons. J Biol Chem 263:2324-2329.

47. Muir H. 1995. The chondrocyte, architect of cartilage. Biomechanics, structure, function and molecular biology of cartilage matrix macromolecules. Bioessays 17:1039-1048.

48. Mow VC, Wang CC, Hung CT. 1999. The extracellular matrix, interstitial fluid and ions as a mechanical signal transducer in articular cartilage. Osteoarthritis and Cartilage 7:41-58.

49. Carney SL, Muir H. 1988. The structure and function of cartilage proteoglycans. Physiol Rev 68:858-910.

50. Rosenberg K, Olsson H, Morgelin M, et al. 1998. Cartilage oligomeric matrix protein shows high affinity zinc-dependent interaction with triple helical collagen. J Biol Chem 273:20397-20403.

51. Pendleton A, Arden N, Dougados M, et al. 2000. EULAR recommendations for the management of knee osteoarthritis: report of a task force of the Standing Committee for International Clinical Studies Including Therapeutic Trials (ESCISIT). Ann Rheum Dis 59:936-944. 
52. Pelletier JP, Martel-Pelletier J, Abramson SB. 2001. Osteoarthritis, an inflammatory disease: potential implication for the selection of new therapeutic targets. Arthritis Rheum 44:1237-1247.

53. Fransen M, McConnell S, Bell M. 2003. Exercise for osteoarthritis of the hip or knee. Cochrane Database Syst Rev CD004286.

54. Bartels EM, Lund H, Hagen KB, et al. 2007. Aquatic exercise for the treatment of knee and hip osteoarthritis. Cochrane Database Syst Rev CD005523.

55. Messier SP, Loeser RF, Miller GD, et al. 2004. Exercise and dietary weight loss in overweight and obese older adults with knee osteoarthritis: the Arthritis, Diet, and Activity Promotion Trial. Arthritis Rheum 50:1501-1510.

56. Lozada CJ, Altman RD. 1997. Chondroprotection in osteoarthritis. Bull Rheum Dis 46:5-7.

57. Towheed TE, Hochberg MC. 1997. A systematic review of randomized controlled trials of pharmacological therapy in osteoarthritis of the knee, with an emphasis on trial methodology. Semin Arthritis Rheum 26:755-770.

58. Towheed TE. 2002. Published meta-analyses of pharmacological therapies for osteoarthritis. Osteoarthritis Cartilage 10:836-837.

59. Deviere J. 2002. Do selective cyclo-oxygenase inhibitors eliminate the adverse events associated with nonsteroidal anti-inflammatory drug therapy? Eur J Gastroenterol Hepatol 14 Suppl 1:S29-33.

60. Sibbald B. 2004. Rofecoxib (Vioxx) voluntarily withdrawn from market. Cmaj 171:1027-1028.

61. Bassleer C, Henrotin Y, Franchimont P. 1992. In-vitro evaluation of drugs proposed as chondroprotective agents. Int J Tissue React 14:231-241.

62. Setnikar I, Pacini MA, Revel L. 1991. Antiarthritic effects of glucosamine sulfate studied in animal models. Arzneimittelforschung 41:542-545.

63. Reginster JY, Bruyere O, Henrotin Y. 2003. New perspectives in the management of osteoarthritis. Structure modification: facts or fantasy? J Rheumatol 30:14-20.

64. Towheed TE, Maxwell L, Anastassiades TP, et al. 2005. Glucosamine therapy for treating osteoarthritis. Cochrane Database Syst Rev CD002946.

65. Clegg DO, Reda DJ, Harris CL, et al. 2006. Glucosamine, chondroitin sulfate, and the two in combination for painful knee osteoarthritis. N Engl J Med 354:795-808.

66. Bellamy N, Campbell J, Robinson V, et al. 2006. Intraarticular corticosteroid for treatment of osteoarthritis of the knee. Cochrane Database Syst Rev CD005328.

67. Bellamy N, Campbell J, Robinson V, et al. 2005. Intraarticular corticosteroid for treatment of osteoarthritis of the knee. Cochrane Database Syst Rev CD005328.

68. Godwin M, Dawes M. 2004. Intra-articular steroid injections for painful knees. Systematic review with meta-analysis. Can Fam Physician 50:241-248.

69. Bellamy N, Campbell J, Robinson V, et al. 2005. Viscosupplementation for the treatment of osteoarthritis of the knee. Cochrane Database Syst Rev CD005321. 
70. Wang CT, Lin J, Chang CJ, et al. 2004. Therapeutic effects of hyaluronic acid on osteoarthritis of the knee. A meta-analysis of randomized controlled trials. J Bone Joint Surg Am 86-A:538-545.

71. Lo GH, LaValley M, McAlindon T, et al. 2003. Intra-articular hyaluronic acid in treatment of knee osteoarthritis: a meta-analysis. Jama 290:3115-3121.

72. Arrich J, Piribauer F, Mad P, et al. 2005. Intra-articular hyaluronic acid for the treatment of osteoarthritis of the knee: systematic review and meta-analysis. Cmaj 172:10391043.

73. Smith MD, Triantafillou S, Parker A, et al. 1997. Synovial membrane inflammation and cytokine production in patients with early osteoarthritis. J Rheumatol 24:365-371.

74. Cameron-Donaldson M, Holland C, Hungerford DS, et al. 2004. Cartilage debris increases the expression of chondrodestructive tumor necrosis factor-alpha by articular chondrocytes. Arthroscopy 20:1040-1043.

75. Moseley JB, O'Malley K, Petersen NJ, et al. 2002. A controlled trial of arthroscopic surgery for osteoarthritis of the knee. N Engl J Med 347:81-88.

76. Pridie KH. 1959. A method of resurfacing osteoarthritic knee joints. Journal of Bone and Joint Surgery. British 41-B:618-619.

77. Dzioba RB. 1988. The classification and treatment of acute articular cartilage lesions. Arthroscopy 4:72-80.

78. Johnson LL. 1986. Arthroscopic abrasion arthroplasty historical and pathologic perspective: present status. Arthroscopy 2:54-69.

79. Johnson LL. 2001. Arthroscopic abrasion arthroplasty: a review. Clin Orthop 391S:S306-317.

80. Johnson-Nurse C, Dandy DJ. 1985. Fracture-separation of articular cartilage in the adult knee. Journal of Bone and Joint Surgery. British 67-B:42-43.

81. Yen YM, Cascio B, O'Brien L, et al. 2008. Treatment of osteoarthritis of the knee with microfracture and rehabilitation. Med Sci Sports Exerc 40:200-205.

82. Blevins FT, Steadman JR, Rodrigo JJ, et al. 1998. Treatment of articular cartilage defects in athletes: an analysis of functional outcome and lesion appearance. Orthopedics 21:761-768.

83. Steadman JR, Miller BS, Karas SG, et al. 2003. The microfracture technique in the treatment of full-thickness chondral lesions of the knee in National Football League players. J Knee Surg 16:83-86.

84. Steadman JR, Rodkey WG, Briggs KK, et al. 1999. [The microfracture technic in the management of complete cartilage defects in the knee joint]. Orthopade 28:26-32.

85. Steadman JR, Briggs KK, Rodrigo JJ, et al. 2003. Outcomes of microfracture for traumatic chondral defects of the knee: average 11-year follow-up. Arthroscopy 19:477-484. 
86. Gudas R, Kalesinskas RJ, Kimtys V, et al. 2005. A prospective randomized clinical study of mosaic osteochondral autologous transplantation versus microfracture for the treatment of osteochondral defects in the knee joint in young athletes. Arthroscopy 21:1066-1075.

87. Kreuz PC, Steinwachs MR, Erggelet C, et al. 2006. Results after microfracture of fullthickness chondral defects in different compartments in the knee. Osteoarthritis Cartilage 14:1119-1125.

88. Ficat RP, Ficat C, Gédéon P, et al. 1979. Spongialization: A new treatment for diseased patellae. Clinical Orthopedics and Related Research 144:74-83.

89. Sharma L, Song J, Felson DT, et al. 2001. The role of knee alignment in disease progression and functional decline in knee osteoarthritis. Jama 286:188-195.

90. Jackson JP, Waugh W. 1961. Tibial osteotomy for osteoarthritis of the knee. J Bone Joint Surg Br 43-B:746-751.

91. Coventry MB, Ilstrup DM, Wallrichs SL. 1993. Proximal tibial osteotomy. Journal of Bone and Joint Surgery. American 75-A:196-201.

92. Naudie D, Bourne RB, Rorabeck CH, et al. 1999. The Install Award. Survivorship of the high tibial valgus osteotomy. A 10- to -22-year followup study. Clin Orthop Relat Res 18-27.

93. Berman AT, Bosacco SJ, Kirshner S, et al. 1991. Factors influencing long-term results in high tibial osteotomy. Clin Orthop Relat Res 192-198.

94. McDevitt CA, Muir H. 1976. Biochemical changes in the cartilage of the knee in experimental and natural osteoarthritis in the dog. J Bone Joint Surg Br 58:94-101.

95. Sprenger TR, Doerzbacher JF. 2003. Tibial osteotomy for the treatment of varus gonarthrosis. Survival and failure analysis to twenty-two years. J Bone Joint Surg Am 85A:469-474.

96. Yasuda K, Majima T, Tsuchida T, et al. 1992. A ten- to 15-year follow-up observation of high tibial osteotomy in medial compartment osteoarthrosis. Clin Orthop Relat Res 186-195.

97. Grelsamer RP. 1995. Unicompartmental osteoarthrosis of the knee. J Bone Joint Surg Am 77:278-292.

98. Hart JA, Sekel R. 2002. Osteotomy of the knee: is there a seat at the table? J Arthroplasty 17:45-49.

99. Ritter MA, Fechtman RA. 1988. Proximal tibial osteotomy. A survivorship analysis. J Arthroplasty 3:309-311.

100. Healy WL, Riley LH, Jr. 1986. High tibial valgus osteotomy. A clinical review. Clin Orthop Relat Res 227-233.

101. Brouwer RW, Raaij van TM, Bierma-Zeinstra SM, et al. 2007. Osteotomy for treating knee osteoarthritis. Cochrane Database Syst Rev CD004019. 
102. Akizuki S, Yasukawa Y, Takizawa T. 1997. Does arthroscopic abrasion arthroplasty promote cartilage regeneration in osteoarthritic knees with eburnation? A prospective study of high tibial osteotomy with abrasion arthroplasty versus high tibial osteotomy alone. Arthroscopy 13:9-17.

103. van Valburg AA, van Roermund PM, Lammens J, et al. 1995. Can Ilizarov joint distraction delay the need for an arthrodesis of the ankle? A preliminary report. J Bone Joint Surg Br 77:720-725.

104. Ploegmakers JJ, van Roermund PM, van Melkebeek J, et al. 2005. Prolonged clinical benefit from joint distraction in the treatment of ankle osteoarthritis. Osteoarthritis Cartilage 13:582-588.

105. Marijnissen AC, van Roermund PM, van Melkebeek J, et al. 2003. Clinical benefit of joint distraction in the treatment of ankle osteoarthritis. Foot Ankle Clin 8:335-346.

106. van Roermund PM, Marijnissen AC, Lafeber FP. 2002. Joint distraction as an alternative for the treatment of osteoarthritis. Foot Ankle Clin 7:515-527.

107. van Valburg AA, van Roermund PM, Marijnissen AC, et al. 1999. Joint distraction in treatment of osteoarthritis: a two-year follow-up of the ankle. Osteoarthritis Cartilage 7:474-479.

108. Czitrom AA, Langer F, McKee N, et al. 1986. Bone and cartilage allotransplantation. Clinical Orthopedics and Related Research 208:141-145.

109. Tomford WW, Duff GP, Mankin HJ. 1985. Experimental freeze-preservation of chondrocytes. Clinical Orthopedics and Related Research 197:11-14.

110. Matsusue Y, Yamamuro T, Hama H. 1993. Arthroscopic multiple osteochondral transplantation to the chondral defect in the knee associated with anterior cruciate ligament disruption. Arthroscopy 9:318-321.

111. Hangody L, Fules P. 2003. Autologous osteochondral mosaicplasty for the treatment of full-thickness defects of weight-bearing joints: ten years of experimental and clinical experience. J Bone Joint Surg Am 85-A Suppl 2:25-32.

112. Hangody L, Kish G, Kárpáti Z, et al. 1998. Mosaicplasty for the treatment of articular cartilage defects: Application in clinical practice. Orthopedics 21:751-756.

113. Imhoff AB, Öttl GM, Burkart A, et al. 1999. Autologous osteochondral transplantation on various joints. Der Orthopäde 28:33-44.

114. Bobic V. 1996. Arthroscopic osteochondral autograft transplantation in anterior cruciate ligament reconstruction: A preliminary clinical study. Knee Surg Sports Traumatology Arthroscopy 3:262-264.

115. Grande DA, Pitman MI, Peterson L, et al. 1989. The repair of experimentally produced defects in rabbit articular cartilage by autologous chondrocyte transplantation. Journal of Orthopaedic Research 7:208-218.

116. Brittberg M, Tallheden T, Sjogren-Jansson B, et al. 2001. Autologous chondrocytes used for articular cartilage repair: an update. Clin Orthop 391S:S337-348. 
117. Brittberg M. 2008. Autologous chondrocyte implantation--technique and long-term follow-up. Injury 39 Suppl 1:S40-49.

118. Mont MA, Jones LC, Vogelstein BN, et al. 1999. Evidence of inappropriate application of autologous cartilage transplantation therapy in an uncontrolled environment. Am J Sports Med 27:617-620.

119. Peterson L, Minas T, Brittberg M, et al. 2000. Two- to 9-year outcome after autologous chondrocyte transplantation of the knee. Clin Orthop 212-234.

120. Peterson L, Minas T, Brittberg M, et al. 2003. Treatment of osteochondritis dissecans of the knee with autologous chondrocyte transplantation: results at two to ten years. J Bone Joint Surg Am 85-A Suppl 2:17-24.

121. Peterson L, Brittberg M, Kiviranta I, et al. 2002. Autologous chondrocyte transplantation. Biomechanics and long-term durability. Am J Sports Med 30:2-12.

122. Beris $A E$, Lykissas MG, Papageorgiou CD, et al. 2005. Advances in articular cartilage repair. Injury 36 Suppl 4:S14-23.

123. Hunziker EB, Stahli A. 2008. Surgical suturing of articular cartilage induces osteoarthritis-like changes. Osteoarthritis Cartilage

124. Niedermann B, Boe S, Lauritzen J, et al. 1985. Glued periosteal grafts in the knee. Acta Orthopaedica Scandinavica 56:457-460.

125. Hoikka VE, Jaroma HJ, Ritsila VA. 1990. Reconstruction of the patellar articulation with periosteal grafts. 4-year follow-up of 13 cases. Acta Orthop Scand 61:36-39.

126. Lorentzon R, Alfredson H, Hildingsson C. 1998. Treatment of deep cartilage defects of the patella with periosteal transplantation. Knee Surg Sports Traumatol Arthrosc 6:202208.

127. O'Driscoll SW. 1999. Articular Cartilage Regeneration Using Periosteum. Clinical Orthopaedics and Related Research 367:S186-S203.

128. Gallay SH, Miura $\mathrm{Y}$, Commisso $\mathrm{CN}$, et al. 1994. Relationship of donor site to chondrogenic potential of periosteum in vitro. J Orthop Res 12:515-525.

129. Homminga GN, Bulstra SK, Bouwmeester PSM, et al. 1990. Perichondral grafting for cartilage lesions of the knee. Journal of Bone and Joint Surgery. British 72-B:1003-1007.

130. Bouwmeester SJM, Beckers JMH, Kuijer R, et al. 1997. Long-term results of rib perichondrial grafts for the repair of cartilage defects in the human knee. International Orthopaedics 21:313-317.

131. Langer R, Vacanti JP. 1993. Tissue engineering. Science 260:920-926.

132. Cherubino P, Grassi FA, Bulgheroni P, et al. 2003. Autologous chondrocyte implantation using a bilayer collagen membrane: a preliminary report. J Orthop Surg (Hong Kong) 11:10-15.

133. Bartlett W, Skinner JA, Gooding CR, et al. 2005. Autologous chondrocyte implantation versus matrix-induced autologous chondrocyte implantation for osteochondral defects of the knee: a prospective, randomised study. J Bone Joint Surg Br 87:640-645. 
134. Marlovits S, Zeller P, Singer P, et al. 2006. Cartilage repair: generations of autologous chondrocyte transplantation. Eur J Radiol 57:24-31.

135. Athanasiou KA, Agrawal CM, Barber FA, et al. 1998. Orthopaedic applications for PLAPGA biodegradable polymers. Arthroscopy 14:726-737.

136. Bonaventure J, Kadhom N, Cohen-Solal L, et al. 1994. Reexpression of cartilagespecific genes by dedifferentiated human articular chondrocytes cultured in alginate beads. Experimental Cell Research 212:97-104.

137. von der Mark K, Gauss B, von der Mark H, et al. 1977. Relationship between cell shape and type of collagen synthesized as chondrocytes lose their cartilage phenotype in culture. Nature 267:531-532.

138. Benya PD, Shaffer JD. 1982. Dedifferentiated Chondrocytes Reexpress the Differentiated Collagen Phenotype when Cultured in Agarose Gels. Cell 30:215-224.

139. Binette F, McQuaid DP, Haudenschild DR, et al. 1998. Expression of a Stable Articular Cartilage Phenotype without Evidence of Hypertrophy by Adult Human Articular Chondrocytes In Vitro. Journal of Orthopaedic Research 16:207-216.

140. Tuli R, Nandi S, Li WJ, et al. 2004. Human mesenchymal progenitor cell-based tissue engineering of a single-unit osteochondral construct. Tissue Eng 10:1169-1179.

141. Deans RJ, Moseley AB. 2000. Mesenchymal stem cells: biology and potential clinical uses. Exp Hematol 28:875-884.

142. Qiao C, Xu W, Zhu W, et al. 2008. Human mesenchymal stem cells isolated from the umbilical cord. Cell Biol Int 32:8-15.

143. Im Gl, Shin YW, Lee KB. 2005. Do adipose tissue-derived mesenchymal stem cells have the same osteogenic and chondrogenic potential as bone marrow-derived cells? Osteoarthritis Cartilage 13:845-853.

144. Tallheden T, Dennis JE, Lennon DP, et al. 2003. Phenotypic plasticity of human articular chondrocytes. J Bone Joint Surg Am 85-A Suppl 2:93-100.

145. Wickham MQ, Erickson GR, Gimble JM, et al. 2003. Multipotent stromal cells derived from the infrapatellar fat pad of the knee. Clin Orthop Relat Res 196-212.

146. Dowthwaite GP, Bishop JC, Redman SN, et al. 2004. The surface of articular cartilage contains a progenitor cell population. J Cell Sci 117:889-897.

147. Barry F, Boynton RE, Liu B, et al. 2001. Chondrogenic differentiation of mesenchymal stem cells from bone marrow: differentiation-dependent gene expression of matrix components. Exp Cell Res 268:189-200.

148. Mackay AM, Beck SC, Murphy JM, et al. 1998. Chondrogenic differentiation of cultured human mesenchymal stem cells from marrow. Tissue Eng 4:415-428.

149. Erickson GR, Gimble JM, Franklin DM, et al. 2002. Chondrogenic potential of adipose tissue-derived stromal cells in vitro and in vivo. Biochem Biophys Res Commun 290:763-769. 
150. Caplan Al. 1991. Mesenchymal Stem Cells. Journal of Orthopaedic Research 9:641650.

151. Nehrer S, Spector M, Minas T. 1999. Histologic Analysis of Tissue After Failed Cartilage Repair Procedures. Clinical Orthopaedics and Related Research 365:149-162.

152. Bouwmeester SJM, Kuijer R, Terwindt-Rouwenhorst EAW, et al. 1999. Histological and biochemical evaluation of perichondrial transplants in human articular cartilage defects. Journal of Orthopaedic Research 17:843-849.

153. Bouwmeester SJM, Kuijer R, Homminga GN, et al. 2002. A Retrospective Analysis of Two Independent Prospective Cartilage Repair Studies: Autogenous Perichondrial grafting versus Subchondral Drilling 10 Years Post-Surgery. Journal of Orthopaedic Research 20:267-273.

154. Bulstra SK, Kuijer R, Eerdmans P, et al. 1994. The effect in vitro of irrigating solutions on intact rat articular cartilage. J Bone Joint Surg Br 76:468-470.

155. Bulstra SK, Douw, C., Kuijer, R.: NaCl irrigation of the rat knee inhibits cartilage metabolism; hyaluronan restores this disturbed metabolism to normal. In: 45th Annual Meeting, Orthopaedic Research Society. Anaheim, California, 1999

156. Malda J, Woodfield TB, van der Vloodt F, et al. 2005. The effect of PEGT/PBT scaffold architecture on the composition of tissue engineered cartilage. Biomaterials 26:63-72.

157. van Dijkhuizen-Radersma R, Peters FL, Stienstra NA, et al. 2002. Control of vitamin B12 release from poly(ethylene glycol)/poly(butylene terephthalate) multiblock copolymers. Biomaterials 23:1527-1536.

158. Du C, Meijer GJ, van de Valk C, et al. 2002. Bone growth in biomimetic apatite coated porous Polyactive 1000PEGT70PBT30 implants. Biomaterials 23:4649-4656.

159. Woodfield TB, Malda J, de Wijn J, et al. 2004. Design of porous scaffolds for cartilage tissue engineering using a three-dimensional fiber-deposition technique. Biomaterials 25:4149-4161.

160. Papadaki M, Mahmood T, Gupta P, et al. 2001. The different behaviors of skeletal muscle cells and chondrocytes on PEGT/PBT block copolymers are related to the surface properties of the substrate. J Biomed Mater Res 54:47-58.

161. Miot S, Woodfield T, Daniels AU, et al. 2005. Effects of scaffold composition and architecture on human nasal chondrocyte redifferentiation and cartilaginous matrix deposition. Biomaterials 26:2479-2489.

162. Treppo S, Koepp H, Quan EC, et al. 2000. Comparison of biomechanical and biochemical properties of cartilage from human knee and ankle pairs. J Orthop Res 18:739-748.

163. Beresford JN. 1989. Osteogenic stem cells and the stromal system of bone and marrow. Clin Orthop Relat Res 270-280.

164. Dorshkind K. 1990. Regulation of hemopoiesis by bone marrow stromal cells and their products. Annu Rev Immunol 8:111-137. 
165. Zuk PA, Zhu M, Mizuno H, et al. 2001. Multilineage cells from human adipose tissue: implications for cell-based therapies. Tissue Eng 7:211-228.

166. O'Driscoll SW, Recklies AD, Poole AR. 1994. Chondrogenesis in periosteal explants. An organ culture model for in vitro study. Journal of Bone and Joint Surgery. American 76-A:1042-1051.

167. Ito Y, Fitzsimmons JS, Sanyal A, et al. 2001. Localization of chondrocyte precursors in periosteum. Osteoarthritis Cartilage 9:215-223.

168. Nakahara H, Bruder SP, Goldberg VM, et al. 1990. In vivo osteochondrogenic potential of cultured cells derived from the periosteum. Clin Orthop 223-232.

169. O'Driscoll SWM, Saris DBF, Ito $Y$, et al. 2001. The chondrogenic potential of periosteum decreases with age. Journal of Orthopaedic Research 19:95-103.

170. O'Driscoll SW, Saris DB, Ito Y, et al. 2001. The chondrogenic potential of periosteum decreases with age. J Orthop Res 19:95-103.

171. Bredella MA, Tirman PF, Peterfy CG, et al. 1999. Accuracy of T2-weighted fast spinecho MR imaging with fat saturation in detecting cartilage defects in the knee: comparison with arthroscopy in 130 patients. AJR Am J Roentgenol 172:1073-1080.

172. Gao J, Knaack D, Goldberg VM, et al. 2004. Osteochondral defect repair by demineralized cortical bone matrix. Clin Orthop Relat Res S62-66.

173. Shao X, Goh JC, Hutmacher DW, et al. 2006. Repair of large articular osteochondral defects using hybrid scaffolds and bone marrow-derived mesenchymal stem cells in a rabbit model. Tissue Eng 12:1539-1551.

174. Mizuta H, Kudo S, Nakamura E, et al. 2006. Expression of the PTH/PTHrP receptor in chondrogenic cells during the repair of full-thickness defects of articular cartilage. Osteoarthritis Cartilage 14:944-952.

175. Lu Y, Markel MD, Swain C, et al. 2006. Development of partial thickness articular cartilage injury in an ovine model. J Orthop Res

176. Hunziker EB, Quinn TM. 2003. Surgical removal of articular cartilage leads to loss of chondrocytes from cartilage bordering the wound edge. J Bone Joint Surg Am 85-A Suppl 2:85-92.

177. Sachs L. 1982. Applied Statistics: A Handbook of Techniques. 2nd ed:

178. Mankin HJ, Dorfman H, Lippiello L, et al. 1971. Biochemical and Metabolic Abnormalities in Articular Cartilage from Osteo-Arthritic Human Hips. Journal of Bone and Joint Surgery. American 53A:523-537.

179. Lafeber FP, Vander Kraan PM, Van Roy JL, et al. 1993. Articular cartilage explant culture; an appropriate in vitro system to compare osteoarthritic and normal human cartilage. Connect Tissue Res 29:287-299.

180. Pelletier JP, Martel-Pelletier J, Howell DS, et al. 1983. Collagenase and collagenolytic activity in human osteoarthritic cartilage. Arthritis Rheum 26:63-68. 
181. Hunziker EB. 1999. Biologic Repair of Articular Cartilage: Defect Models in Experimental Animals and Matrix Requirements. Clinical Orthopaedics and Related Research 367:S135-S146.

182. Brandt KD, Myers SL, Burr D, et al. 1991. Osteoarthritic Changes in Canine Articular Cartilage, Subchondral Bone, and Synovium 54 Months After Transection of the Anterior Cruciate Ligament. Arthritis and Rheumatism 34:1560-1570.

183. Han CD, Kang HJ. 1990. Changes in the hyaline articular cartilage after air exposure. Yonsei Med J 31:53-59.

184. Speer KP, Callaghan JJ, Seaber AV, et al. 1990. The effects of exposure of articular cartilage to air. A histochemical and ultrastructural investigation. J Bone Joint Surg Am 72:1442-1450.

185. Mitchell N, Shepard N. 1989. The deleterious effects of drying on articular cartilage. J Bone Joint Surg Am 71:89-95.

186. Bauer MS, Woodard JC, Weigel JP. 1986. Effects of exposure to ambient air on articular cartilage of rabbits. Am J Vet Res 47:1268-1270.

187. Lefkoe TP, Trafton PG, Ehrlich MG, et al. 1993. An experimental model of femoral condylar defect leading to osteoarthrosis. Journal of Orthopaedics and Traumatology 7:458-467.

188. Walker EA. 2000. Cellular Responses of Embryonic Hyaline Cartilage to Experimental Wounding In Vitro. Journal of Orthopaedic Research 18:25-34.

189. Hunt N, Sanchez-Ballester J, Pandit R, et al. 2001. Chondral lesions of the knee: A new localization method and correlation with associated pathology. Arthroscopy 17:481-490.

190. Visco DM, Hill MA, Widmer WR, et al. 1996. Experimental osteoarthritis in dogs: a comparison of the Pond-Nuki and medial arthrotomy methods. Osteoarthritis and Cartilage 4:9-22.

191. Johnson JM, Johnson AL. 1993. Cranial cruciate ligament rupture. Pathogenesis, diagnosis, and postoperative rehabilitation. Vet Clin North Am Small Anim Pract 23:717-733.

192. Marijnissen AC, van Roermund PM, TeKoppele JM, et al. 2002. The canine 'groove' model, compared with the ACLT model of osteoarthritis. Osteoarthritis Cartilage 10:145-155.

193. Laurent TC, Fraser JR. 1992. Hyaluronan. Faseb J 6:2397-2404.

194. Balazs EA, Denlinger JL. 1993. Viscosupplementation: a new concept in the treatment of osteoarthritis. J Rheumatol Suppl 39:3-9.

195. Watterson JR, Esdaile JM. 2000. Viscosupplementation: therapeutic mechanisms and clinical potential in osteoarthritis of the knee. J Am Acad Orthop Surg 8:277-284.

196. Mathies B. 2006. Effects of Viscoseal, a synovial fluid substitute, on recovery after arthroscopic partial meniscectomy and joint lavage. Knee Surg Sports Traumatol Arthrosc 14:32-39. 
197. Bos PK, DeGroot J, Budde M, et al. 2002. Specific enzymatic treatment of bovine and human articular cartilage: implications for integrative cartilage repair. Arthritis Rheum 46:976-985.

198. Kim HA, Lee YJ, Seong S-C, et al. 2000. Apoptotic Chondrocyte Death in Human Osteoarthritis. Journal of Rheumatology 27:455-462.

199. Bos PK, DeGroot J, Budde M, et al. 2002. Specific enzymatic treatment of bovine and human articular cartilage: Implications for integrative cartilage repair. Arthritis Rheum 46:976-985.

200. de Vries BJ, van den Berg WB, Vitters E, et al. 1986. Quantitation of glycosaminoglycan metabolism in anatomically intact articular cartilage of the mouse patella: in vitro and in vivo studies with $35 \mathrm{~S}$-sulfate, $3 \mathrm{H}$-glucosamine, and $3 \mathrm{H}$-acetate. Rheumatology International 6:273-281.

201. Verschure PJ, Joosten LA, van der Kraan PM, et al. 1994. Responsiveness of articular cartilage from normal and inflamed mouse knee joints to various growth factors. Ann Rheum Dis 53:455-460.

202. Diaz-Gallego L, Prieto JG, Coronel P, et al. 2005. Apoptosis and nitric oxide in an experimental model of osteoarthritis in rabbit after hyaluronic acid treatment. J Orthop Res 23:1370-1376.

203. D'Lima DD, Hashimoto S, Chen PC, et al. 2001. Prevention of chondrocyte apoptosis. J Bone Joint Surg Am 83-A Suppl 2:25-26.

204. Lisignoli G, Grassi F, Zini N, et al. 2001. Anti-Fas-induced apoptosis in chondrocytes reduced by hyaluronan: evidence for CD44 and CD54 (intercellular adhesion molecule 1) invovement. Arthritis Rheum 44:1800-1807.

205. Mendelson S, Wooley P, Lucas D, et al. 2004. The effect of hyaluronic acid on a rabbit model of full-thickness cartilage repair. Clin Orthop Relat Res 266-271.

206. Lu Y, Markel MD, Swain C, et al. 2006. Development of partial thickness articular cartilage injury in an ovine model. J Orthop Res 24:1974-1982.

207. Bulstra SK, Kuijer R, Eerdmans P, et al. 1994. The effect in vitro of irrigating solutions on intact rat articular cartilage. Journal of Bone and Joint Surgery. British 76-B:468-470.

208. Bulstra SK, Douw, C., Kuijer, R.: NaCl irrigation of the rat knee inhibits cartilage metabolism; hyaluronan restores this disturbed metabolism to normal. In: 45th Annual Meeting, Orthopaedic Research Society. Anaheim, California

209. Gelber AC, Hochberg MC, Mead LA, et al. 2000. Joint injury in young adults and risk for subsequent knee and hip osteoarthritis. Ann Intern Med 133:321-328.

210. Mankin HJ. 1974. The reaction of articular cartilage to injury and osteoarthritis (second of two parts). N Engl J Med 291:1335-1340.

211. Mankin HJ. 1974. The reaction of articular cartilage to injury and osteoarthritis (first of two parts). New England Journal of Medicine 291:1285-1292. 
212. Altman RD, Kates J, Chun LE, et al. 1992. Preliminary Observations of Chondral Abrasion in a Canine Model. Annals of the Rheumatic Diseases 51:1056-1062.

213. Mitchell N, Shepard N. 1976. The resurfacing of adult rabbit articular cartilage by multiple perforation through the subchondral bone. Journal of Bone and Joint Surgery. American 58-A:230-233.

214. Martin I, Miot S, Barbero A, et al. 2006. Osteochondral tissue engineering. J Biomech

215. Bentley G. 1992. Articular Tissue Grafts. Annals of the Rheumatic Diseases 51:292-296.

216. Brittberg M, Faxén E, Peterson L. 1994. Carbon Fiber Scaffolds in the Treatment of Early Knee Osteoarthritis. Clinical Orthopedics and Related Research 307:155-164.

217. Messner K. 1994. Durability of artificial implants for repair of osteochondral defects of the medial femoral condyle in rabbits. Biomaterials 15:657-664.

218. Messner K, Gillquist J. 1993. Synthetic implants for the repair of osteochondral defects of the medial femoral condyle: a biomechanical and histological evaluation in the rabbit knee. Biomaterials 14:513-521.

219. Hunziker EB. 1999. Articular artilage repair: are the intrinsic biological constraints undermining this process insuperable? Osteoarthritis and Cartilage 7:15-28.

220. Mahmood TA, Shastri VP, van Blitterswijk CA, et al. 2006. Evaluation of chondrogenesis within PEGT: PBT scaffolds with high PEG content. J Biomed Mater Res A 79:216-222.

221. Lamme EN, Druecke D, Pieper J, et al. 2007. Long-term Evaluation of Porous PEGT/PBT Implants for Soft Tissue Augmentation. J Biomater Appl

222. O'Driscoll SW, Keeley FW, Salter RB. 1986. The chondrogenic potential of free autogenous periosteal grafts for biological resurfacing of major full-thickness defects in joint surfaces under the influence of continuous passive motion. An experimental investigation in the rabbit. J Bone Joint Surg Am 68:1017-1035.

223. Niederauer GG, Slivka MA, Leatherbury NC, et al. 2000. Evaluation of multiphase implants for repair of focal osteochondral defects in goats. Biomaterials 21:2561-2574.

224. Räsänen T, Messner K. 1996. Regional variations of indentation stiffness and thickness of normal rabbit knee articular cartilage. Journal of Biomedical Materials Research 31:519-524.

225. Kelly DJ, Prendergast PJ. 2006. Prediction of the optimal mechanical properties for a scaffold used in osteochondral defect repair. Tissue Eng 12:2509-2519.

226. Schipani E. 2005. Hypoxia and HIF-1 alpha in chondrogenesis. Semin Cell Dev Biol 16:539-546.

227. Jansen EJ, Sladek RE, Bahar H, et al. 2005. Hydrophobicity as a design criterion for polymer scaffolds in bone tissue engineering. Biomaterials 26:4423-4431.

228. Mahmood TA, Miot S, Frank O, et al. 2006. Modulation of chondrocyte phenotype for tissue engineering by designing the biologic-polymer carrier interface. Biomacromolecules 7:3012-3018. 
229. Mahmood TA, de Jong R, Riesle J, et al. 2004. Adhesion-mediated signal transduction in human articular chondrocytes: the influence of biomaterial chemistry and tenascinC. Exp Cell Res 301:179-188.

230. Deschamps AA, Claase MB, Sleijster WJ, et al. 2002. Design of segmented poly(ether ester) materials and structures for the tissue engineering of bone. J Control Release 78:175-186.

231. Holland TA, Bodde EW, Baggett LS, et al. 2005. Osteochondral repair in the rabbit model utilizing bilayered, degradable oligo(poly(ethylene glycol) fumarate) hydrogel scaffolds. J Biomed Mater Res A 75:156-167.

232. Swieszkowski W, Tuan BH, Kurzydlowski KJ, et al. 2007. Repair and regeneration of osteochondral defects in the articular joints. Biomol Eng

233. Oliveira JM, Rodrigues MT, Silva SS, et al. 2006. Novel hydroxyapatite/chitosan bilayered scaffold for osteochondral tissue-engineering applications: Scaffold design and its performance when seeded with goat bone marrow stromal cells. Biomaterials 27:6123-6137.

234. Schek RM, Taboas JM, Segvich SJ, et al. 2004. Engineered osteochondral grafts using biphasic composite solid free-form fabricated scaffolds. Tissue Eng 10:1376-1385.

235. Moroni L, Hendriks JA, Schotel R, et al. 2007. Design of biphasic polymeric 3dimensional fiber deposited scaffolds for cartilage tissue engineering applications. Tissue Eng 13:361-371.

236. Gelber AC, Hochberg MC, Mead LA, et al. 2000. Joint injury in young adults and risk for subsequent knee and hip osteoarthritis. Ann.Intern.Med. 133:321-328.

237. Mankin HJ. 1974. The reaction of articular cartilage to injury and osteoarthritis (second of two parts). New England Journal of Medicine 291:1335-1340.

238. Erggelet C, Sittinger M, Lahm A. 2003. The arthroscopic implantation of autologous chondrocytes for the treatment of full-thickness cartilage defects of the knee joint. Arthroscopy 19:108-110.

239. Andereya S, Maus U, Gavenis K, et al. 2006. First clinical experiences with a novel 3Dcollagen gel (CaReS) for the treatment of focal cartilage defects in the knee. Z Orthop Ihre Grenzgeb 144:272-280.

240. Ossendorf C, Kaps C, Kreuz PC, et al. 2007. Treatment of posttraumatic and focal osteoarthritic cartilage defects of the knee with autologous polymer-based threedimensional chondrocyte grafts: 2-year clinical results. Arthritis Res.Ther. 9:R41.

241. Pulliainen O, Vasara Al, Hyttinen MM, et al. 2007. Poly-L-D-lactic acid scaffold in the repair of porcine knee cartilage lesions. Tissue Engineering 13:1347-1355.

242. Brittberg M, Peterson L, Sjogren-Jansson E, et al. 2003. Articular cartilage engineering with autologous chondrocyte transplantation. A review of recent developments. Journal of Bone and Joint Surgery-American Volume 85-A Suppl 3:109-115. 
243. Ossendorf C, Kreuz PC, Steinwachs MR, et al. 2007. Autologous chondrocyte implantation for the treatment of large full-thickness cartilage lesions of the knee. Saudi.Med.J. 28:1251-1256.

244. Lee CR, Grodzinsky AJ, Hsu HP, et al. 2000. Effects of harvest and selected cartilage repair procedures on the physical and biochemical properties of articular cartilage in the canine knee. Journal of Orthopaedic Research 18:790-799.

245. Barry F, Boynton RE, Liu B, et al. 2001. Chondrogenic differentiation of mesenchymal stem cells from bone marrow: differentiation-dependent gene expression of matrix components. Experimental Cell Research 268:189-200.

246. Erickson GR, Gimble JM, Franklin DM, et al. 2002. Chondrogenic potential of adipose tissue-derived stromal cells in vitro and in vivo. Biochem.Biophys.Res.Commun. 290:763-769.

247. Mackay AM, Beck SC, Murphy JM, et al. 1998. Chondrogenic differentiation of cultured human mesenchymal stem cells from marrow. Tissue Engineering 4:415-428.

248. Huang Jl, Kazmi N, Durbhakula MM, et al. 2005. Chondrogenic potential of progenitor cells derived from human bone marrow and adipose tissue: a patient-matched comparison. J.Orthop.Res. 23:1383-1389.

249. Liu TM, Martina M, Hutmacher DW, et al. 2007. Identification of common pathways mediating differentiation of bone marrow- and adipose tissue-derived human mesenchymal stem cells into three mesenchymal lineages. Stem Cells 25:750-760.

250. Winter A, Breit S, Parsch D, et al. 2003. Cartilage-like gene expression in differentiated human stem cell spheroids: A comparison of bone marrow-derived and adipose tissuederived stromal cells. Arthritis and Rheumatism 48:418-429.

251. Pittenger MF, Mackay AM, Beck SC, et al. 1999. Multilineage Potential of Adult Human Mesenchymal Stem Cells. Science 284:143-146.

252. Beresford JN. 1989. Osteogenic stem cells and the stromal system of bone and marrow. Clin.Orthop.Relat Res. 240:270-280.

253. Dorshkind K. 1990. Regulation of hemopoiesis by bone marrow stromal cells and their products. Annu.Rev.Immunol. 8:111-137.

254. Zuk PA, Zhu M, Mizuno H, et al. 2001. Multilineage cells from human adipose tissue: implications for cell- based therapies. Tissue Engineering 7:211-228.

255. Ito Y, Fitzsimmons JS, Sanyal A, et al. 2001. Localization of chondrocyte precursors in periosteum. Osteoarthritis and Cartilage 9:215-223.

256. Nakahara H, Bruder SP, Goldberg VM, et al. 1990. In vivo osteochondrogenic potential of cultured cells derived from the periosteum. Clinical orthopaedics and related research 223-232.

257. O'Driscoll SW, Recklies AD, Poole AR. 1994. Chondrogenesis in periosteal explants. An organ culture model for in vitro study. Journal of Bone and Joint Surgery-American Volume 76-A:1042-1051. 
258. Iwasaki M, Nakata K, Nakahara H, et al. 1993. Transforming growth factor-beta 1 stimulates chondrogenesis and inhibits osteogenesis in high density culture of periosteum-derived cells. Endocrinology 132:1603-1608.

259. Iwasaki M, Nakahara H, Nakata K, et al. 1995. Regulation of proliferation and osteochondrogenic differentiation of periosteum-derived cells by transforming growth factor-beta and basic fibroblast growth factor. J.Bone Joint Surg.Am. 77:543-554.

260. Miura Y, Fitzsimmons JS, Commisso CN, et al. 1994. Enhancement of periosteal chondrogenesis in vitro. Dose-response for transforming growth factor-beta 1 (TGF-beta 1). Clinical orthopaedics and related research 271-280.

261. Nakahara H, Goldberg VM, Caplan Al. 1991. Culture-Expanded Human PeriostealDerived Cells Exhibit Osteochondral Potential In Vivo. Journal of Orthopaedic Research 9:465-476.

262. De Bari C, Dell'Accio F, Luyten FP. 2001. Human periosteum-derived cells maintain phenotypic stability and chondrogenic potential throughout expansion regardless of donor age. Arthritis and Rheumatism 44:85-95.

263. De Bari C, Dell'Accio F, Vanlauwe J, et al. 2006. Mesenchymal multipotency of adult human periosteal cells demonstrated by single-cell lineage analysis. Arthritis Rheum. 54:1209-1221.

264. O'Driscoll SW, Saris DB, Ito Y, et al. 2001. The chondrogenic potential of periosteum decreases with age. J.Orthop.Res. 19:95-103.

265. Hunt N, Sanchez-Ballester J, Pandit R, et al. 2001. Chondral lesions of the knee: A new localization method and correlation with associated pathology. Arthroscopy 17:481490.

266. Gilbert SF, Migeon BR. 1975. D-valine as a selective agent for normal human and rodent epithelial cells in culture. Cell 5:11-17.

267. Armati PJ, Constable AL, Llewellyn F. 1990. A new medium for in vitro peripheral nervous tissue myelination without the use of antimitotics. J.Neurosci.Methods 33:149155.

268. Frauli M, Ludwig H. 1987. Inhibition of fibroblast proliferation in a culture of human endometrial stromal cells using a medium containing D-valine. Arch.Gynecol.Obstet. 241:87-96.

269. Piquette GN, Timms BG. 1990. Isolation and characterization of rabbit ovarian surface epithelium, granulosa cells, and peritoneal mesothelium in primary culture. In Vitro Cell Dev.Biol. 26:471-481.

270. Fitzsimmons JS, Sanyal A, Gonzalez C, et al. 2004. Serum-free media for periosteal chondrogenesis in vitro. J.Orthop.Res. 22:716-725.

271. Meuleman N, Tondreau T, Delforge A, et al. 2006. Human marrow mesenchymal stem cell culture: serum-free medium allows better expansion than classical alpha-MEM medium. Eur.J.Haematol. 76:309-316. 
272. Vukicevic S, Luyten FP, Reddi AH. 1989. Stimulation of the expression of osteogenic and chondrogenic phenotypes in vitro by osteogenin. Proceedings of the National Academy of Sciences of the United States of America 86:8793-8797.

273. Mandl EW, van der Veen SW, Verhaar JA, et al. 2002. Serum-Free Medium Supplemented with High-Concentration FGF2 for Cell Expansion Culture of Human Ear Chondrocytes Promotes Redifferentiation Capacity. Tissue Engineering 8:573-580.

274. Hauselmann HJ, Aydelotte MB, Schumacher BL, et al. 1992. Synthesis and turnover of proteoglycans by human and bovine adult articular chondrocytes cultured in alginate beads. Matrix 12:116-129.

275. Kuijer R, Surtel DAM, Passier RCJJ, et al. 1998. A novel method to examine the phenotype of chondrocytes. Journal of Materials Science: Materials in Medicine 9:749-754.

276. Dharmavaram RM, Baldwin CT, Reginato AM, et al. 1993. Amplification of cDNAs for human cartilage-specific types II, IX and XI collagens from chondrocytes and EpsteinBarr virus-transformed lymphocytes. Matrix 13:125-133.

277. Reichenberger E, Beier F, Luvalle P, et al. 1992. Genomic Organization and Full-Length cDNA Sequence of Human Collagen-X. FEBS Letters 311:305-310.

278. Choi YS, Noh SE, Lim SM, et al. 2008. Multipotency and growth characteristic of periosteum-derived progenitor cells for chondrogenic, osteogenic, and adipogenic differentiation. Biotechnol.Lett. 30:593-601.

279. Lim SM, Choi YS, Shin HC, et al. 2005. Isolation of human periosteum-derived progenitor cells using immunophenotypes for chondrogenesis. Biotechnol.Lett. 27:607-611.

280. Lennon DP, Haynesworth SE, Young RG, et al. 1995. A chemically defined medium supports in vitro proliferation and maintains the osteochondral potential of rat marrowderived mesenchymal cells. Experimental Cell Research 219:211-222.

281. Stevens M, Qanadilo H, Langer R, et al. 2002. FGF-2 in combination with TGF-b1 enhances periosteum derived chondrogenesis in vitro. 4th ICRS symposium SM01199894.

282. Solchaga LA, Penick K, Porter JD, et al. 2005. FGF-2 enhances the mitotic and chondrogenic potentials of human adult bone marrow-derived mesenchymal stem cells. J.Cell Physiol 203:398-409.

283. Walsh S, Jefferiss C, Stewart K, et al. 2000. Expression of the developmental markers STRO-1 and alkaline phosphatase in cultures of human marrow stromal cells: regulation by fibroblast growth factor (FGF)-2 and relationship to the expression of FGF receptors 1-4. Bone 27:185-195.

284. Zaragosi LE, Ailhaud G, Dani C. 2006. Autocrine fibroblast growth factor 2 signaling is critical for self-renewal of human multipotent adipose-derived stem cells. Stem Cells 24:2412-2419.

285. Walsh S, Jefferiss CM, Stewart K, et al. 2003. IGF-I does not affect the proliferation or early osteogenic differentiation of human marrow stromal cells. Bone 33:80-89. 
286. Lange C, Cakiroglu F, Spiess AN, et al. 2007. Accelerated and safe expansion of human mesenchymal stromal cells in animal serum-free medium for transplantation and regenerative medicine. J.Cell Physiol 213:18-26.

287. Müller I, Kordowich S, Holzwarth C, et al. 2006. Animal serum-free culture conditions for isolation and expansion of multipotent mesenchymal stromal cells from human BM. Cytotherapy. 8:437-444.

288. Wang WG, Lou SQ, Ju XD, et al. 2003. In vitro chondrogenesis of human bone marrow-derived mesenchymal progenitor cells in monolayer culture: activation by transfection with TGF-beta2. Tissue \& Cell 35:69-77.

289. Huang CY, Reuben PM, D'Ippolito G, et al. 2004. Chondrogenesis of human bone marrow-derived mesenchymal stem cells in agarose culture. Anat.Rec.A Discov.Mol.Cell Evol.Biol. 278:428-436.

290. Indrawattana N, Chen G, Tadokoro M, et al. 2004. Growth factor combination for chondrogenic induction from human mesenchymal stem cell. Biochemical and Biophysical Research Communications 320:914-919.

291. Choi YS, Lim SM, Shin HC, et al. 2007. Chondrogenesis of human periosteum-derived progenitor cells in atelocollagen. Biotechnol.Lett. 29:323-329.

292. Fukumoto T, Sperling JW, Sanyal A, et al. 2003. Combined effects of insulin-like growth factor-1 and transforming growth factor-beta1 on periosteal mesenchymal cells during chondrogenesis in vitro. Osteoarthritis Cartilage 11:55-64.

293. Mierisch CM, Cohen SB, Jordan LC, et al. 2002. Transforming growth factor-beta in calcium alginate beads for the treatment of articular cartilage defects in the rabbit. Arthroscopy 18:892-900.

294. Kafienah W, Mistry S, Perry MJ, et al. 2007. Pharmacological regulation of adult stem cells: chondrogenesis can be induced using a synthetic inhibitor of the retinoic acid receptor. Stem Cells 25:2460-2468.

295. Estes BT, Wu AW, Guilak F. 2006. Potent induction of chondrocytic differentiation of human adipose-derived adult stem cells by bone morphogenetic protein 6 . Arthritis Rheum. 54:1222-1232.

296. Hennig T, Lorenz H, Thiel A, et al. 2007. Reduced chondrogenic potential of adipose tissue derived stromal cells correlates with an altered TGFbeta receptor and BMP profile and is overcome by BMP-6. J.Cell Physiol 211:682-691.

297. Brownlow HC, Reed A, Joyner C, et al. 2000. Anatomical effects of periosteal elevation. J.Orthop.Res. 18:500-502.

298. Emans PJ, Surtel DAM, Frings EJJ, et al. 2005. In vivo generation of cartilage out of periosteum. Tissue Engineering 11:369-377.

299. Elek SD, Conen PE. 1957. The virulence of Staphylococcus pyogenes for man; a study of the problems of wound infection. Br J Exp Pathol 38:573-586. 
300. Lidwell OM, Lowbury EJ, Whyte W, et al. 1983. Airborne contamination of wounds in joint replacement operations: the relationship to sepsis rates. J Hosp Infect 4:111-131.

301. Zimmerli W, Waldvogel FA, Vaudaux P, et al. 1982. Pathogenesis of foreign body infection: description and characteristics of an animal model. J Infect Dis 146:487-497.

302. Lavik E, Langer R. 2004. Tissue engineering: current state and perspectives. Appl Microbiol Biotechnol 65:1-8.

303. Levenberg S, Langer R. 2004. Advances in tissue engineering. Curr Top Dev Biol 61:113-134.

304. Vacanti JP, Langer R. 1999. Tissue engineering: the design and fabrication of living replacement devices for surgical reconstruction and transplantation. Lancet 354 Suppl 1:SI32-34.

305. Gristina AG. 1987. Biomaterial-centered infection: microbial adhesion versus tissue integration. Science 237:1588-1595.

306. Gristina AG, Naylor P, Myrvik Q. 1988. Infections from biomaterials and implants: a race for the surface. Med Prog Technol 14:205-224.

307. Claase MB, Grijpma DW, Mendes SC, et al. 2003. Porous PEOT/PBT scaffolds for bone tissue engineering: preparation, characterization, and in vitro bone marrow cell culturing. J Biomed Mater Res A 64:291-300.

308. Woodfield TBF, Bezemer JM, Pieper JS, et al. 2002. Scaffolds for tissue engineering of cartilage. Crit Rev Eukaryot Gene Expr 12:209-236.

309. El-Ghalbzouri A, Lamme EN, van Blitterswijk C, et al. 2004. The use of PEGT/PBT as a dermal scaffold for skin tissue engineering. Biomaterials 25:2987-2996.

310. Malda J, Woodfield TB, van der Vloodt F, et al. 2004. The effect of PEGT/PBT scaffold architecture on oxygen gradients in tissue engineered cartilaginous constructs. Biomaterials 25:5773-5780.

311. Moroni L, de Wijn JR, van Blitterswijk CA. 2005. Three-dimensional fiber-deposited PEOT/PBT copolymer scaffolds for tissue engineering: influence of porosity, molecular network mesh size, and swelling in aqueous media on dynamic mechanical properties. J Biomed Mater Res A 75:957-965.

312. Bakker D, van Blitterswijk CA, Daems WT, et al. 1988. Biocompatibility of six elastomers in vitro. Journal of Biomedical Materials Research 22:423-439.

313. Beumer GJ, van Blitterswijk CA, Ponec M. 1994. Biocompatibility of a biodegradable matrix used as a skin substitute: an in vivo evaluation. Journal of Biomedical Materials Research 28:545-552.

314. Bouwmeester SJM, Kuijer R, Sollie-Drees MMWE, et al. 1998. Quantitative histological analysis of bony ingrowth within the biomaterial PolyactiveTM implanted in different bone locations: An experimental study in rabbits. Journal of Materials Science: Materials in Medicine 9:181-185. 
315. Gaillard ML, van den Brink J, van Blitterswijk CA, et al. 1994. Applying a calcium phosphate layer on PEO/PBT copolymers affects bone formation in vivo. Journal of Materials Science: Materials in Medicine 5:424-428.

316. Radder AM, Leenders H, van Blitterswijk CA. 1995. Bone-bonding behaviour of poly(ethylene oxide)-polybutylene terephthalate copolymer coatings and bulk implants: a comparative study. Biomaterials 16:507-513.

317. van Blitterswijk CA, van der Brink J, Leenders $H$, et al. 1993. The effect of PEO ratio on degradation, calcification and bone bonding of PEO/PBT copolymer (Polyactive). Cells and Materials 3:23-36.

318. van Haastert RM, Grote JJ, van Blitterswijk CA, et al. 1994. Osteoinduction within $\mathrm{PEO} / \mathrm{PBT}$ copolymer implants in cranial defects using demineralized bone matrix. Journal of Materials Science: Materials in Medicine 5:764-769.

319. Emans PJ, Pieper J, Hulsbosch MM, et al. 2006. Differential cell viability of chondrocytes and progenitor cells in tissue-engineered constructs following implantation into osteochondral defects. Tissue Eng 12:1699-1709.

320. Freed LE, Vunjak-Novakovic G, Langer R. 1993. Cultivation of cell-polymer cartilage implants in bioreactors. Journal of Cellular Biochemistry 51:257-264.

321. Freed LE, Hollander AP, Martin I, et al. 1998. Chondrogenesis in a cell-polymerbioreactor system. Experimental cell research 240:58-65.

322. Antti-Poika I, Josefsson G, Konttinen Y, et al. 1990. Hip arthroplasty infection. Current concepts. Acta Orthop Scand 61:163-169.

323. Harris WH, Sledge CB. 1990. Total hip and total knee replacement (1). N Engl J Med 323:725-731.

324. Engelsman AF, van der Mei HC, Ploeg RJ, et al. 2007. The phenomenon of infection with abdominal wall reconstruction. Biomaterials 28:2314-2327.

325. Leber GE, Garb JL, Alexander Al, et al. 1998. Long-term complications associated with prosthetic repair of incisional hernias. Arch Surg 133:378-382.

326. Boelens JJ, Dankert J, Murk JL, et al. 2000. Biomaterial-associated persistence of Staphylococcus epidermidis in pericatheter macrophages. J Infect Dis 181:1337-1349.

327. Boelens JJ, Zaat SA, Meeldijk J, et al. 2000. Subcutaneous abscess formation around catheters induced by viable and nonviable Staphylococcus epidermidis as well as by small amounts of bacterial cell wall components. J Biomed Mater Res 50:546-556.

328. Drenkard E, Ausubel FM. 2002. Pseudomonas biofilm formation and antibiotic resistance are linked to phenotypic variation. Nature 416:740-743.

329. Neut D, van Horn JR, van Kooten TG, et al. 2003. Detection of biomaterial-associated infections in orthopaedic joint implants. Clin Orthop Relat Res 261-268.

330. Maathuis PG, Neut D, Busscher HJ, et al. 2005. Perioperative contamination in primary total hip arthroplasty. Clin Orthop Relat Res 136-139. 
331. Bakker D, Van Blitterswijk CA, Daems WT, et al. 1988. Biocompatibility of six elastomers in vitro. J Biomed Mater Res 22:423-439.

332. Hesseling SC, Grote JJ, Bakker D, et al. 1992. Clinical performance and biocompatibility analysis of tympanic implants.

333. Beumer GJ, van Blitterswijk CA, Ponec M. 1994. Degradative behaviour of polymeric matrices in (sub)dermal and muscle tissue of the rat: a quantitative study. Biomaterials 15:551-559.

334. An YH, Kang QK, Arciola CR. 2006. Animal models of osteomyelitis. Int J Artif Organs 29:407-420.

335. Borzsei L, Mintal T, Koos Z, et al. 2006. Examination of a novel, specified local antibiotic therapy through polymethylmethacrylate capsules in a rabbit osteomyelitis model. Chemotherapy 52:73-79.

336. Makinen TJ, Veiranto M, Lankinen $P$, et al. 2005. In vitro and in vivo release of ciprofloxacin from osteoconductive bone defect filler. J Antimicrob Chemother 56:1063-1068.

337. Norden CW. 1988. Lessons learned from animal models of osteomyelitis. Rev Infect Dis 10:103-110.

338. Rissing JP. 1990. Animal models of osteomyelitis. Knowledge, hypothesis, and speculation. Infect Dis Clin North Am 4:377-390.

339. Poelstra KA, Barekzi NA, Grainger DW, et al. 2000. A novel spinal implant infection model in rabbits. Spine 25:406-410.

340. Harris WH, Sledge CB. 1990. Total hip and total knee replacement (2). N Engl J Med 323:801-807.

341. Moroni L, Licht R, de Boer J, et al. 2006. Fiber diameter and texture of electrospun PEOT/PBT scaffolds influence human mesenchymal stem cell proliferation and morphology, and the release of incorporated compounds. Biomaterials 27:4911-4922.

342. Woodfield TB, Miot S, Martin I, et al. 2006. The regulation of expanded human nasal chondrocyte re-differentiation capacity by substrate composition and gas plasma surface modification. Biomaterials 27:1043-1053.

343. Wilson CJ, Clegg RE, Leavesley DI, et al. 2005. Mediation of biomaterial-cell interactions by adsorbed proteins: a review. Tissue Eng 11:1-18.

344. Herrmann M, Vaudaux PE, Pittet D, et al. 1988. Fibronectin, fibrinogen, and laminin act as mediators of adherence of clinical staphylococcal isolates to foreign material. J Infect Dis 158:693-701.

345. Hill E, Boontheekul T, Mooney DJ. 2006. Designing scaffolds to enhance transplanted myoblast survival and migration. Tissue Eng 12:1295-1304.

346. Smith MK, Riddle KW, Mooney DJ. 2006. Delivery of hepatotrophic factors fails to enhance longer-term survival of subcutaneously transplanted hepatocytes. Tissue Eng 12:235-244. 
347. Sharma B, Elisseeff JH. 2004. Engineering structurally organized cartilage and bone tissues. Ann Biomed Eng 32:148-159.

348. Hutmacher DW. 2000. Scaffolds in tissue engineering bone and cartilage. Biomaterials 21:2529-2543.

349. Capito RM, Spector M. 2003. Scaffold-based articular cartilage repair. IEEE Eng Med Biol Mag 22:42-50.

350. Wiedmann-Al-Ahmad M, Gutwald R, Lauer G, et al. 2002. How to optimize seeding and culturing of human osteoblast-like cells on various biomaterials. Biomaterials 23:3319-3328.

351. Holy CE, Shoichet MS, Davies JE. 2000. Engineering three-dimensional bone tissue in vitro using biodegradable scaffolds: investigating initial cell-seeding density and culture period. J Biomed Mater Res 51:376-382.

352. Bergsma JE, de Bruijn WC, Rozema FR, et al. 1995. Late degradation tissue response to poly(L-lactide) bone plates and screws. Biomaterials 16:25-31.

353. Agrawal CM, Athanasiou KA. 1997. Technique to control pH in vicinity of biodegrading PLA-PGA implants. J Biomed Mater Res 38:105-114.

354. Homicz MR, Chia SH, Schumacher BL, et al. 2003. Human septal chondrocyte redifferentiation in alginate, polyglycolic acid scaffold, and monolayer culture. Laryngoscope 113:25-32.

355. Ye Q, Zund G, Benedikt P, et al. 2000. Fibrin gel as a three dimensional matrix in cardiovascular tissue engineering. Eur J Cardiothorac Surg 17:587-591.

356. Aldenhoff YB, Knetsch ML, Hanssen JH, et al. 2004. Coils and tubes releasing heparin. Studies on a new vascular graft prototype. Biomaterials 25:3125-3133.

357. Thomson RC, Wake MC, Yaszemski MJ, et al. 1995. Biodegradable polymer scaffolds to regenerate organs. Advances in Polymer Science 122:245-274.

358. Thomson RC, Mikos AG, Beahm E, et al. 1999. Guided tissue fabrication from periosteum using preformed biodegradable polymer scaffolds. Biomaterials 20:2007-2018.

359. Hanssen HHL, Wetzels GMR, Benzina A, et al. 1999. Metallic Wires with an Adherent Lubricious and Blood-Compatible Polymeric Coating and Their Use in the Manufacture of Novel Slippery-When-Wet Guidewires: Possible Applications Related to Controlled Local Drug Delivery. Journal of Biomedical Materials Research 48:820-828.

360. Nimni ME, Bernick S, Ertl D, et al. 1988. Ectopic bone formation is enhanced in senescent animals implanted with embryonic cells. Clin Orthop Relat Res 255-266.

361. Benzina A, Kruft M-AB, van der Veen FH, et al. 1996. A versatile three-iodine molecular building block leading to new radiopaque polymeric biomaterials. Journal of Biomedical Materials Research 32:459-466.

362. Bruining MJ, Blaauwgeers HGT, Kuijer R, et al. 2000. Tailoring of New Polymeric Biomaterials for the Repair of Medium-Sized Corneal Perforations. BioMacromolecules 1:418-423. 
363. Friedman M. 1937. The use of ranks to avoid the assumption of normality implicit in the analysis of variance. J. Am. Statist. Ass. 32:675-701.

364. Bahar H, Yaffe A, Binderman I. 2003. The influence of nacre surface and its modification on bone apposition: a bone development model in rats. J Periodontol 74:366-371.

365. Yaffe A, Iztkovich M, Earon Y, et al. 1997. Local delivery of an amino bisphosphonate prevents the resorptive phase of alveolar bone following mucoperiosteal flap surgery in rats. J Periodontol 68:884-889.

366. Odian G: Principles of polymerization., Wiley., 1991

367. Pijls RT, Hanssen HH, Nuijts RM, et al. 2004. Flexible coils with a drug-releasing hydrophilic coating: A new platform for controlled delivery of drugs to the eye? Biomed Mater Eng 14:383-393.

368. Bellamy N, Campbell J, Robinson V, et al. 2006. Viscosupplementation for the treatment of osteoarthritis of the knee. Cochrane Database Syst Rev CD005321.

369. Lapcik LJaL, Lapcik L, De Smedt S, et al. 1998. Hyaluronan: Preparation, Structure, Properties, and Applications. Chem Rev 98:2663-2684.

370. Karlsson J, Sjogren LS, Lohmander LS. 2002. Comparison of two hyaluronan drugs and placebo in patients with knee osteoarthritis. A controlled, randomized, double-blind, parallel-design multicentre study. Rheumatology (Oxford) 41:1240-1248.

371. Raman R, Dutta A, Day N, et al. 2008. Efficacy of Hylan G-F 20 and Sodium Hyaluronate in the treatment of osteoarthritis of the knee - A prospective randomized clinical trial. Knee

372. Jakobsen RB, Engebretsen L, Slauterbeck JR. 2005. An analysis of the quality of cartilage repair studies. J Bone Joint Surg Am 87:2232-2239.

373. Magnussen RA, Dunn WR, Carey JL, et al. 2008. Treatment of focal articular cartilage defects in the knee: a systematic review. Clin Orthop Relat Res 466:952-962.

374. Horas U, Pelinkovic D, Herr G, et al. 2003. Autologous chondrocyte implantation and osteochondral cylinder transplantation in cartilage repair of the knee joint. A prospective, comparative trial. J Bone Joint Surg Am 85-A:185-192.

375. Bentley G, Biant LC, Carrington RW, et al. 2003. A prospective, randomised comparison of autologous chondrocyte implantation versus mosaicplasty for osteochondral defects in the knee. J Bone Joint Surg Br 85:223-230.

376. Knutsen G, Drogset JO, Engebretsen L, et al. 2007. A randomized trial comparing autologous chondrocyte implantation with microfracture. Findings at five years. J Bone Joint Surg Am 89:2105-2112.

377. Knutsen G, Engebretsen L, Ludvigsen TC, et al. 2004. Autologous chondrocyte implantation compared with microfracture in the knee. A randomized trial. J Bone Joint Surg Am 86-A:455-464.

378. Cole BJ. 2008. A randomized trial comparing autologous chondrocyte implantation with microfracture. J Bone Joint Surg Am 90:1165; author reply 1165-1166. 
379. Saris DB, Vanlauwe J, Victor J, et al. 2008. Characterized chondrocyte implantation results in better structural repair when treating symptomatic cartilage defects of the knee in a randomized controlled trial versus microfracture. Am J Sports Med 36:235246.

380. Chen H SJ, Chevrier A, Lascau-Coman V, Ouyang W, Hoemann CD, McKee M, Shive MS, Buschmann MD: A comparative study of microfracture and drilling surgical techniques for cartilage repair. In: 54th Annual Meeting of the Orthopaedic Research Society. San Fransisco, 2008

381. Sohn DH, Lottman LM, Lum LY, et al. 2002. Effect of gravity on localization of chondrocytes implanted in cartilage defects. Clin Orthop Relat Res 254-262.

382. Lendlein A, Langer R. 2002. Biodegradable, elastic shape-memory polymers for potential biomedical applications. Science 296:1673-1676.

383. Thornton AJ, Alsberg E, Albertelli M, et al. 2004. Shape-defining scaffolds for minimally invasive tissue engineering. Transplantation 77:1798-1803.

384. Hendriks J, Riesle J, van Blitterswijk CA. 2007. Co-culture in cartilage tissue engineering. J Tissue Eng Regen Med 1:170-178.

385. Emans PJ, Surtel DA, Frings EJ, et al. 2005. In vivo generation of cartilage from periosteum. Tissue Eng 11:369-377.

386. Lu Y, Dhanaraj S, Wang Z, et al. 2006. Minced cartilage without cell culture serves as an effective intraoperative cell source for cartilage repair. J Orthop Res 24:1261-1270. 


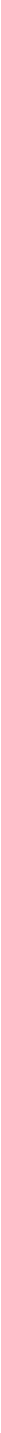


Dankwoord 


\section{DANKWOORD}

Als Assistent Geneeskunde in Opleiding tot Klinisch Onderzoeker (AGIKO) heb ik 3 jaar onderzoek kunnen verrichten, naast de 6 jaar die voor de specialisatie orthopedie staan. In deze periode heb ik veel mensen ontmoet, die me op hun manier hebben geholpen bij het tot stand komen van dit proefschrift.

....Ooit, en meerdere malen, heb ik tegen mezelf en anderen gezegd: "Was ik er maar nooit aan begonnen", en "als het me lukt, dan komt er een groot feest met DJ Jean", en "als me het lukt, dan zal ik zo gelukkig zijn". Inmiddels is het dan zover, en ben ik zo blij dat het gelukt is. Een zeer leerzame ervaring, die ik een ieder aan kan raden. Alle reden om dit te vieren (met D), maar zonder Jean), en een aantal personen te bedanken....

Sjoerd Bulstra. Je bent ontzettend gedreven en enthousiast, en vooral lekker gewoon. De anatomie van de konijnenknie heb jij me bijgebracht, waarna je vanaf de zijlijn het wel en wee van het promotie onderzoek hebt aangeschouwd en perfect hebt gedirigeerd. Bedankt!

Roel Kuijer. Samen op dat kleine kamertje waarin 2 bureaus waren gepropt, begon het allemaal. Je hebt ontzettend veel kennis van kraakbeen, en veel vriendjes in het kraakbeenwereldje. Een prima basis voor het begeleiden van een promovendus. Helaas zijn Sjoerd en jij vertrokken naar het hoge Noorden. Hoe zou ik nu moeten kunnen promoveren zonder directe begeleiding en 349.3 kilometer tussen ons in? Gelukkig is het allemaal goedgekomen, en heb ik niets te klagen gehad over jouw begeleiding vanuit Groningen. Hartstikke bedankt hiervoor.

Geert Walenkamp. Als student was ik altijd een beetje bang voor jou. Zo groot en serieus. Inmiddels ken ik je meerdere jaren, en zaten we veelal op een lijn. Nog steeds ben je groot, maar lang niet altijd serieus. Je hebt me ontzettend geholpen bij de afronding van het proefschrift. Bedankt voor jouw hulp.

Lodewijk van Rhijn. Het begon allemaal met een artikel over Bakerse cysten bij kinderen. De samenwerking verliep prima. Daarom samen nog maar enkele artikels over scoliose geschreven. Inmiddels is jouw promotie al weer jaren achter de (scoliotische) rug, en ben jij één van mijn copromotores. Jouw enthousiasme in de orthopedie en het onderzoek heb je perfect weten over te dragen. Bedankt hiervoor. Ik hoop dat we in de nabije toekomst nog een keer samen wat kunnen schrijven. 
De leden van de beoordelingscommissie, Prof. dr. Van Mameren, dr. Cleutjens, Prof. dr. Dhert, Prof. dr. Geesink en Prof. dr. Geusens, wil ik bedanken voor het beoordelen van het manuscript, en natuurlijk ook het goedkeuren hiervan.

Ruud Geesink. Jij bent diegene die mij het vertrouwen heeft gegeven, en me heeft aangenomen als AGIKO. Bedankt hiervoor. Daarbij voel ik me vereerd dat jij, als o.a. nieuwe kraakbeen deskundige bij Stryker, in de beoordelingscommissie zit.

Pieter Emans. In jouw dankwoord schreef je dat ik in meerdere opzichten een voorbeeld voor je ben. Nou....dat ben je ook in vele opzichten voor mij! Twee jaar later begonnen dan ik, en inmiddels een jaar gepromoveerd. Je bent niet alleen mijn kraakbeen maatje, maar ook een prima collega en goede vriend geworden. Ben blij dat je mijn paranimf wilt zijn. Ik hoop dat we in de nabije toekomst nog veel met elkaar zullen samenwerken.

Bart Wijers. Bedankt dat je mijn paranimf wilt zijn! Je bent geen arts, maar hebt waarschijnlijk meer verstand van polymeren dan welke orthopeed dan ook. Als een van mijn beste vrienden ben ik erg blij dat je naast me wilt staan op 7 november.

Vakgroep Orthopedie Maastricht. Het onderzoeksklimaat in het azM is prima. Ik heb het hier altijd grandioos naar mijn zin gehad. Jullie hebben mij de ruimte gegeven om met regelmaat aan het onderzoek te werken. Daarbij wil ik de enkele nog-niet-gepromoveerden van de vakgroep veel succes wensen met hún promotie.

Patrick Deckers, ik wil je er toch even uitlichten. Bedankt voor alle leuke momenten die we samen hadden in het azM en daarbuiten; o.a. het organiseren van het NOV jaarcongres in Maastricht; de BBQ's in jouw tuin; de festivals; en de liefde voor muziek die we samen delen. 
Don Surtel. Ik heb ontzettend veel aan jou gehad. In 2000 nog alleen met Roel op het Orthopedie lab; inmiddels heb je vele enthousiaste collegae. Van begin tot eind heb je aan mijn zijde gestaan. Met $z^{\prime} n$ tweeën stonden we in de diepvries onze eerste scaffolds uit Teflon buizen te snijden, of maakten ons wekelijks uitstapje naar de konijnen. Heel veel dank voor het vertrouwen, en jouw ongekende rust die je uitstraalde.

Martine Hulsbosch en Mireille Schrooten-van Helden kwamen er later bij, en brachten veel pit en plezier met zich mee. Lachen, gieren en brullen tijdens de operaties en op het lab. Maar ook celkweken voor, achter, boven, onder, en in het kwadraat. Bedankt voor al het werk wat jullie hebben verricht.

Raymond Sladek. Als stagiaire van de Technische Universiteit Eindhoven was je enkele maanden bij ons op het lab. In die tijd heb je me perfect geholpen met mijn onderzoek. Met de onderzoeksresultaten die je hebt meegenomen uit Israël, heb ik van hoofdstuk 8 een compleet verhaal kunnen schrijven.

Tim Welting, je hebt het orthopedie lab naar hogere sferen gebracht. Samen met Don en Andy Cremers vorm je een hecht en enthousiast team, dat altijd voor alles en iedereen klaar staat. Veel studenten zijn al door jullie begeleid, en nog meer staan in de rij om alles te weten over het mysterieuze kraakbeen.

Marion Gijbels, hartstikke bedankt voor het samen beoordelen en scoren van de vele coupes.

Ook wil ik iedereen van de poli, afdeling C4, assistenten Orthopedie, en het secretariaat Orthopedie bedanken. Extra dank voor Barbara Berg en Marion Bastings. Als er weer een brief naar het College van Decanen moest, of bij de correspondentie naar sponsoren; jullie stonden altijd voor me klaar om te helpen met de brieven.

Nick Guldemond, bedankt voor het beantwoorden van de vele vragen op het gebied van de statistiek. Ook ben ik je zeer dankbaar voor de statistische analyses die je veelal in de avonduren en weekenden voor me hebt verricht.

Afdeling Biomaterialen van de Universiteit Maastricht. Samen deelden we de koffiekamer met jullie. Als broekie in de grote onderzoekswereld voelde ik me mede dankzij jullie al snel op mijn plaats. Leo Koole, jouw gedrevenheid is door niemand te evenaren. Je hebt me wegwijs gemaakt in de biomaterialen. Veel dank hiervoor. 
Els Terwindt-Rouwenhorst en Paul van Dijk van de vakgroep Anatomie en Embryologie. Dank voor alle tijd die jullie vrijgemaakt hebben om mij het coupes snijden en kleuren te leren. Wat een engelengeduld hebben jullie.......

Joyce Suyk, Monique de Jong, Frans Slangen, en May Bost van de centrale proefdiervoorziening wil ik bedanken voor hulp tijdens en rondom de operaties. Schitterend om te zien hoe begaan jullie waren met mijn ratten en konijnen.

Partners van het BTS programma. Jens Riesle, Jeanine Hendriks, en Jacoline Zilverentant (allen oud-medewerkers van IsoTis) wil ik bedanken voor de prettige samenwerking. Gerjo van Osch, Erik Mandl, en Koen Bos (afdeling Orthopedie van het Erasmus Medisch centrum Rotterdam), de congressen samen met jullie zijn onvergetelijk.

Maatschap Orthopedie Sittard. Als Agnio hoopte ik al dat ik ooit naar Sittard zou terugkeren. Ik vind het fantastisch dat ik binnenkort als orthopeed in Sittard mag beginnen. Zelden zo'n hardwerkende en leuke groep bij elkaar gezien. Tot gauw!

Adrianus Moonen, dank dat je met jouw ongeschoren been model wilde staan voor de voorkant van mijn proefschrift, zodat Wilmar de Goede hiervan een prachtige foto kon maken. Sjors en Wilmar, bedankt!

Pap en mam. Bedankt dat jullie mij het mogelijk hebben gemaakt geneeskunde te gaan studeren, en uiteindelijk zelfs te promoveren. Waarschijnlijk nooit gedacht dat "enne van Jansen" dat zou doen.

Gerrie en Wim Bogie. Als Isa en Kas thuis waren op mijn onderzoeksdag, waren jullie er altijd om op ze te passen. Zo kon ik boven op het zolderkamertje aan mijn promotie werken. Heel erg bedankt hiervoor.

Het is onmogelijk om iedereen hier persoonlijk te bedanken. Maar zeker mogen niet vergeten worden, alle vrienden die voor de broodnodige ontspanning hebben gezorgd. Dank je wel! 
Zoals hierboven beschreven heb ik dit proefschrift te danken aan meerdere personen. Maar er is slechts één die me altijd heeft aangemoedigd en bijgestaan..... Nicole, mijn Niekske, alleen had ik dit nooit gered! Jij bent mijn aller-allerliefste. Samen met onze schatjes Isa en Kas zijn we het gelukkigste gezin van de wereld. 

Curriculum Vitae 
Edwin Jansen was born in Boxmeer on January 13, 1973. He attended primary school at 't Ogelijn, and the havo and vwo at the Elzendaalcollege in Boxmeer until graduation in 1992. Medicine was studied at the University of Maastricht. After graduation in 1999 he started his medical career as a resident orthopedic surgery in the Maaslandziekenhuis Sittard (dr. A.D. Verburg). In 2000 he made his first steps in cartilage tissue engineering as an AGIKO at the Maastricht University Medical Center. Basic training in surgery was performed at the Atrium Medisch Centrum Parkstad in 2002 and 2003 (Prof. dr. P.R.G. Brink, prof. dr. C.J. Van der Linden). In 2004 he continued his residency at the department of Orthopedic Surgery at the Maastricht University Medical Center (prof. dr. R.G.T. Geesink, dr. A. Van Ooy, prof. dr. G.H.I.M. Walenkamp) and Maaslandziekenhuis Sittard (dr. A.D. Verburg). After his residency (February 2009) he's going to work in the Maaslandziekenhuis Sittard. Edwin and Nicole have 2 children. Isa was born in 2005; Kas in 2007. 


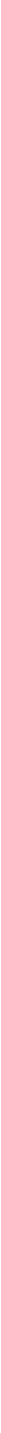


Colour Figures 


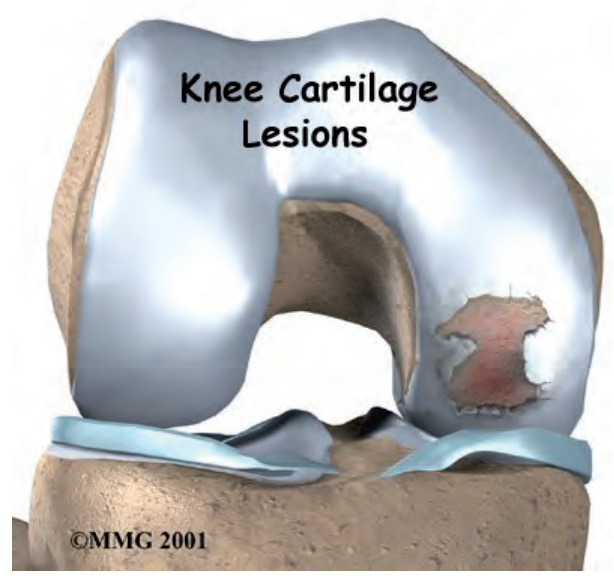

Figure 1. Articular cartilage defect on femoral condyle. Image courtesy of Medical Multimedia Group LLC, www.eOrthopod.com

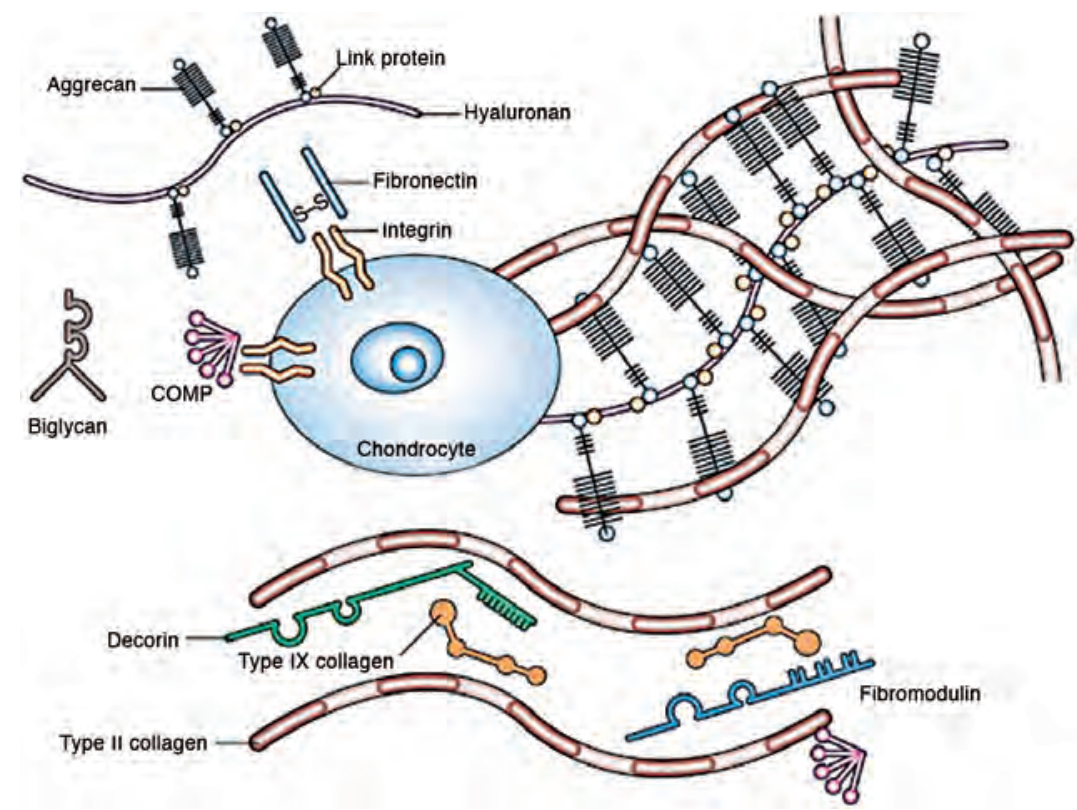

Figure 2. Extracellular matrix of cartilage. Adapted by permission from Macmillan Publishers Ltd: Chen $\mathrm{FH}$ et al. Technology Insight: adult stem cells in cartilage regeneration and tissue engineering. Nature Clinical Practice Rheumatology;2:373-382, copyright 2006. 
Chapter 1

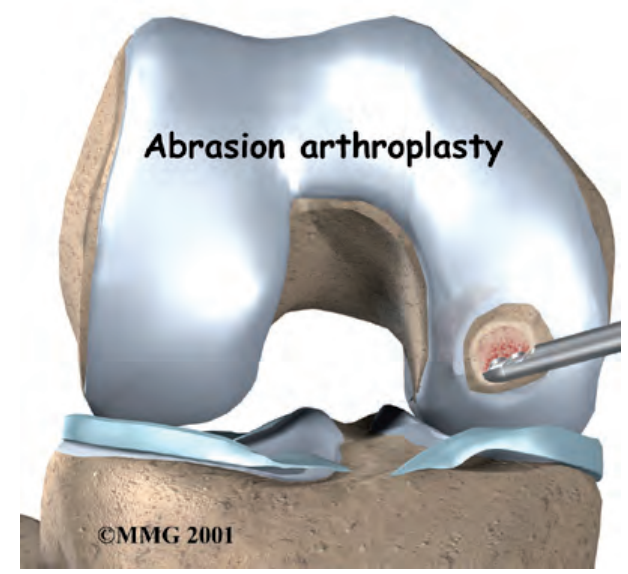

Figure 3. Abrasion arthroplasty. Image courtesy of Medical Multimedia Group LLC, www.eOrthopod.com

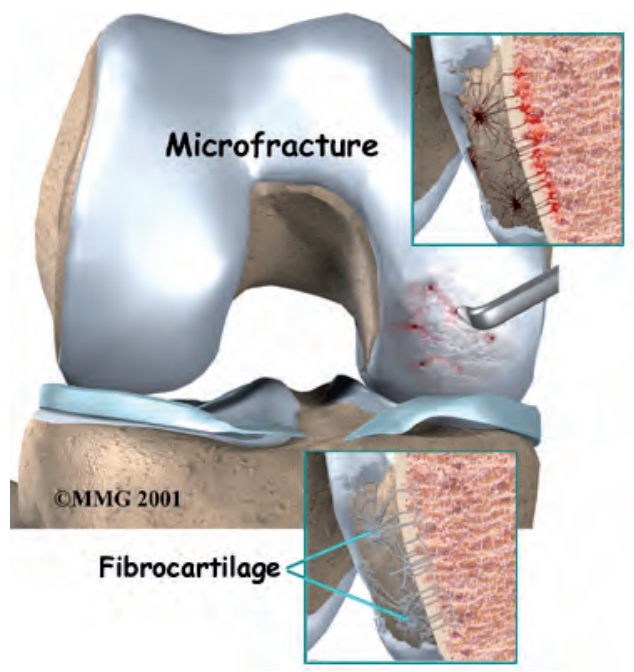

Figure 4. Microfracture. Image courtesy of Medical Multimedia Group LLC, www.eOrthopod.com 


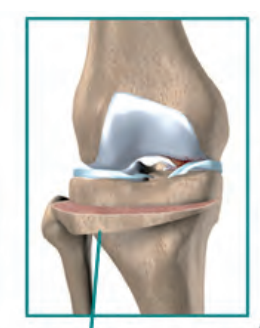

Wedge removed

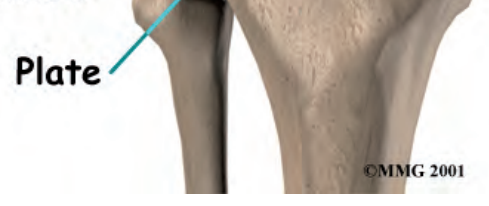

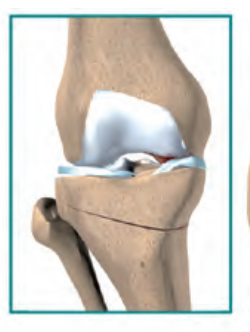

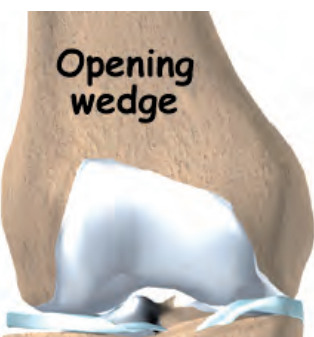
Bone graft inserted

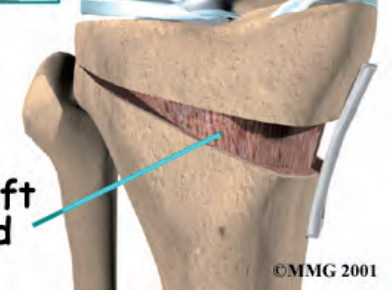

Figure 5. Correction osteotomies. Image courtesy of Medical Multimedia Group LLC, www.eOrthopod.com

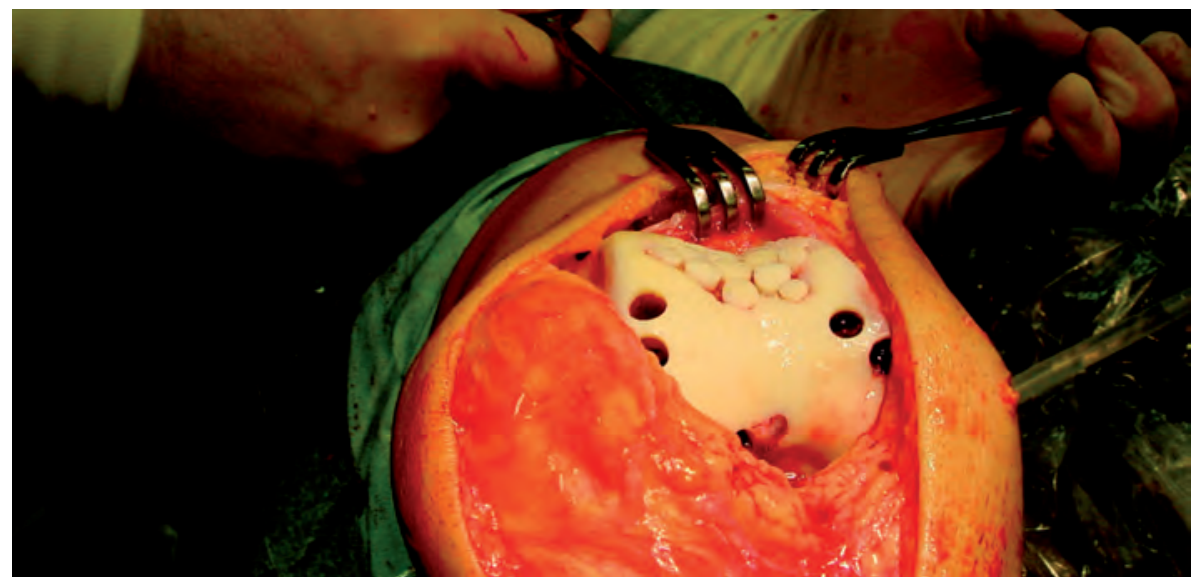

Figure 6. Mosaicplasty. Reprinted with permission from Hangody L and Fules P. Autologous Osteochondral Mosaicplasty for the Treatment of Full-Thickness Defects of Weight-Bearing Joints: Ten Years of Experimental and Clinical Experience. J. Bone Joint Surg. Am., 2003: 8525-32. 


\section{Chapter 3}
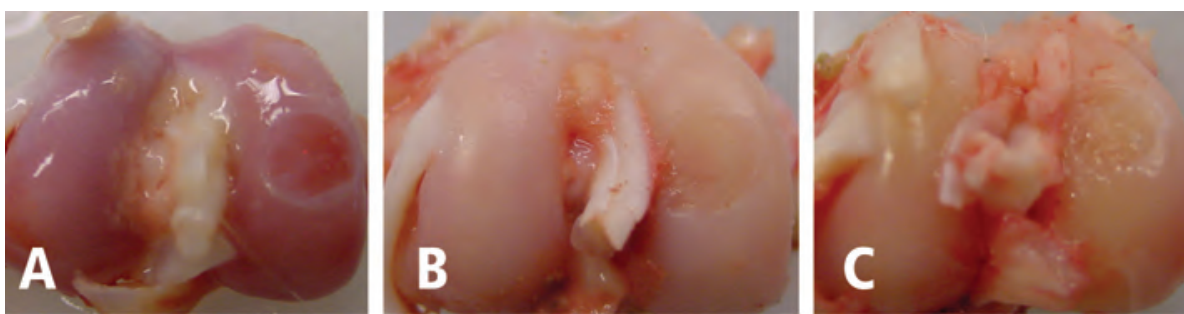

Figure 1. Representative photographs are shown of articular surfaces at (A) 1 week, (B) 13 weeks, and (C) 26 weeks after creating partial-thickness articular cartilage defects on rabbit medial femoral condyles. Cartilage surrounding the defect had a glossy, white, smooth appearance at 1 week, which disappeared during the course of 26 weeks. 


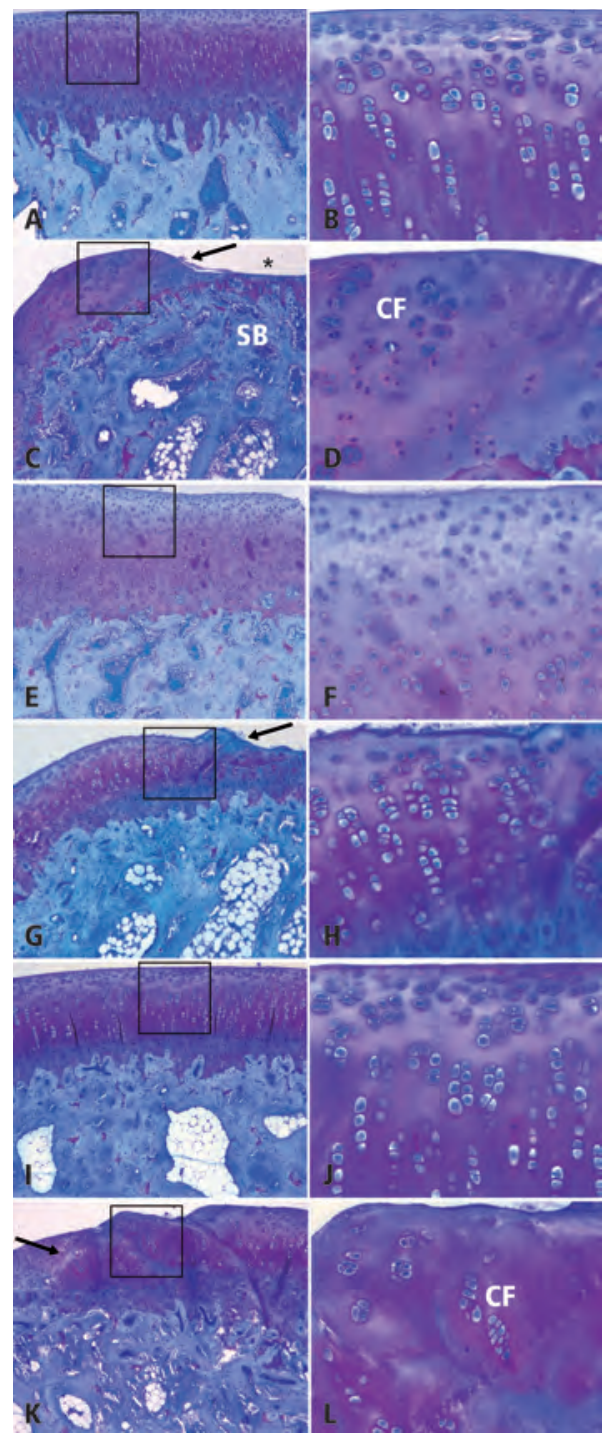

Figure 2. Photomicrographs of sections are shown at (A-D) 1 week, (E-H) 13 weeks, and (I-L) 26 weeks. (A) A sham-treated rabbit medial femoral condyle at 1 week follow-up (Stain, thionine; original magnification, $\times 100$ ); (B) an enlargement of the box in (A) (Stain, thionine; original magnification, $\times 400)$; and (C) a condyle with partialthickness articular cartilage defect at 1 week follow-up are shown (Stain, thionine; original magnification, $\times 100)$. The cartilage defect $(*)$ did not penetrate the subchondral bone (SB); (D) An enlargement of the box in (C) is shown (Stain, thionine; original magnification, $\times 400$ ). A cluster formation (CF) can be seen. (E) A sham-treated rabbit medial femoral condyle at 13 weeks follow-up (Stain, thionine; original magnification, $\times 100)$; (F) an enlargement of the box in (E) (Stain, thionine; original magnification, $\times 400$ ); (G) a condyle with a partial-thickness articular cartilage defect at 13 weeks follow-up (Stain, thionine; original magnification, $\times 100)$; and $(\mathrm{H})$ an enlargement of the box in (G) are shown (Stain, thionine; original magnification, $\times 400$ ). (I) A sham-treated rabbit medial femoral condyle at 26 weeks follow-up (Stain, thionine; original magnification, $\times 100$ ); (J) an enlargement of the box in (I) (Stain, thionine; original magnification, $\times 400)$; and $(\mathrm{K})$ a condyle with partial-thickness articular cartilage defect at 26 weeks follow-up are shown (Stain, thionine; original magnification, $\times 100$ ). The partial-thickness articular cartilage defect was not healed at 26 weeks. Cartilage surrounding the defect showed surface irregularities; (L) an enlargement of the box in $(K)$ is shown (Stain, thionine; original magnification, $\times 400$ ). A cluster formation (CF) can be seen. The arrows in (C), (G), and (K) indicate the edge of the defect. 


\section{Chapter 4}

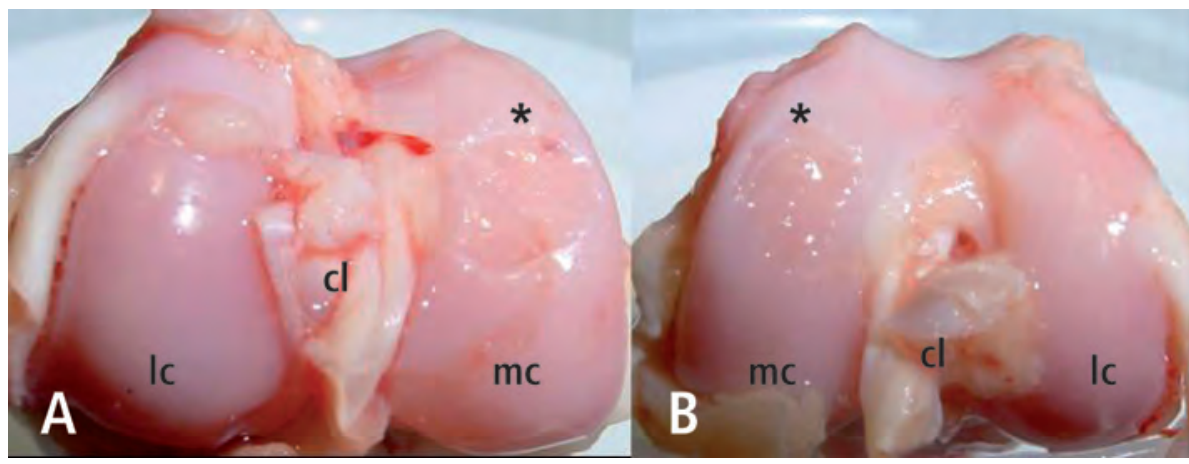

Figure 1. Representative photographs are shown from articular surfaces 2 days after creating partial-thickness articular cartilage defects $\left(^{*}\right)$ on rabbit medial femoral condyles. (A) injected with hyaluronan after irrigation with $0.9 \% \mathrm{NaCl}$ immediately after creating the defects, or (B) irrigated with $0.9 \% \mathrm{NaCl}$. Note that in both hyaluronan- and $\mathrm{NaCl}$-treated knees the surface is smooth and glossy without gross osteoarthritic features. Abbreviations: mc, medial femoral condyle; lc, lateral femoral condyle; $\mathrm{Cl}$, cruciate ligaments. 

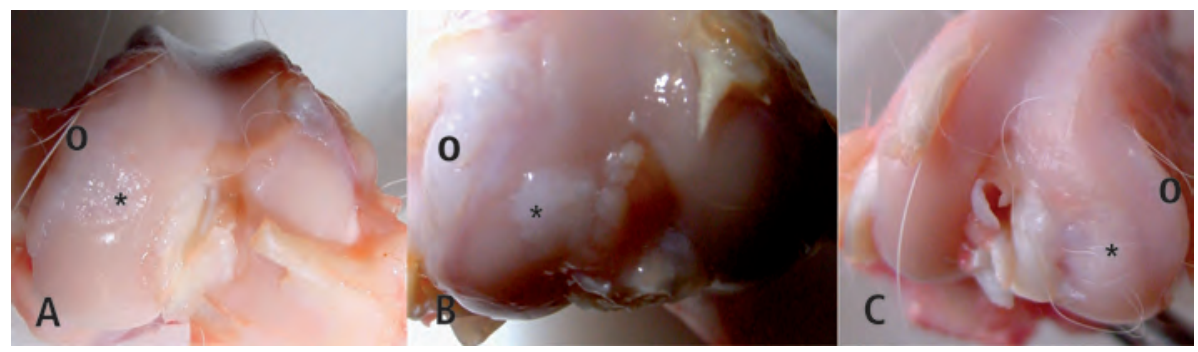

Figure 1. Representative photographs are shown of rabbit knees 3 months after creating osteochondral defects $\left(^{*}\right)$ in medial femoral condyles. Osteochondral defects were left empty (A); or were filled immediately with 55/45 (B) or 70/30 (C) scaffolds. Note the osteophytes (o) on the ridge of femoral condyles.

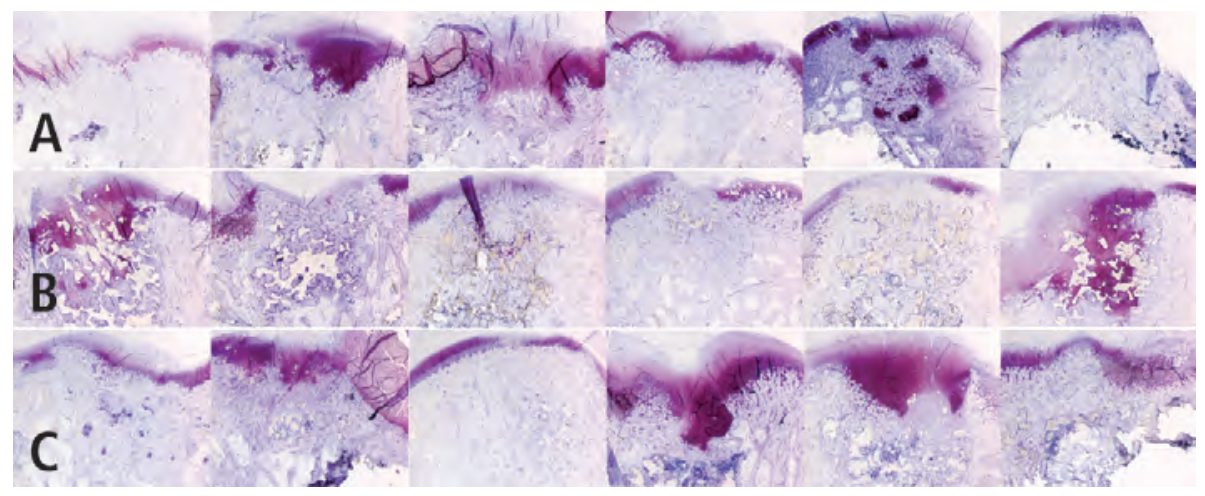

Figure 2. Light micrographs are shown of medial femoral condyles 3 months after creating osteochondral defects.
A. Untreated osteochondral defects (stain, thionine; original magnification, X25)
B. 55/45 treated osteochondral defects (stain, thionine; original magnification, X25)
C. 70/30 treated osteochondral defects (stain, thionine; original magnification, X25) 


\section{Chapter 5}

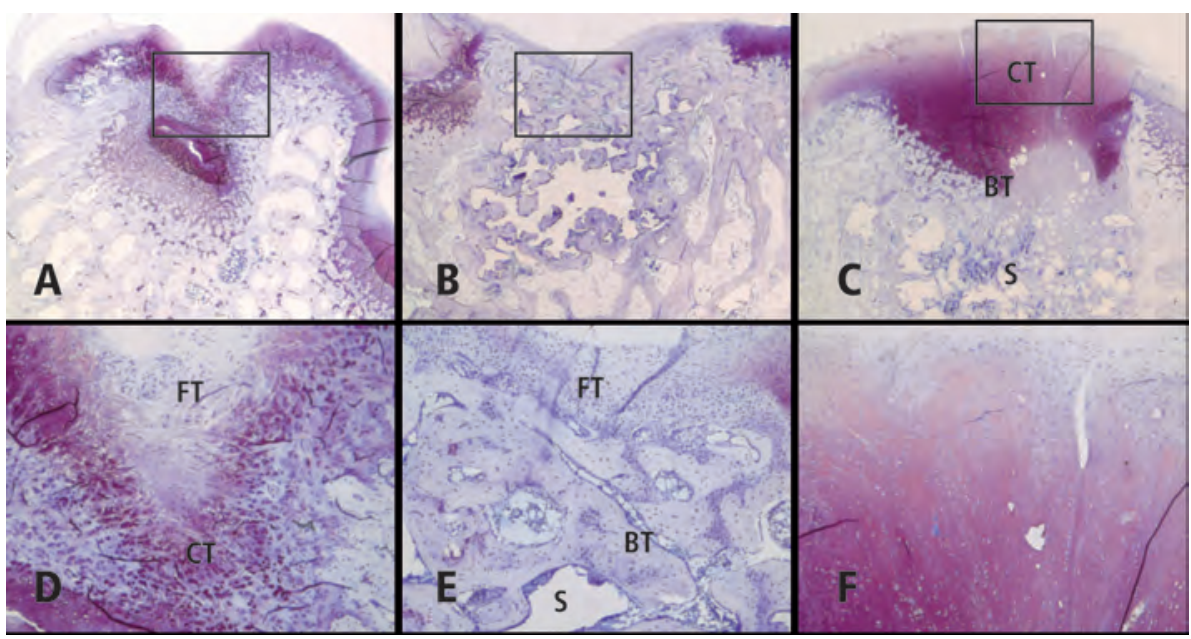

Figure 3. Light micrographs are shown of medial femoral condyles 3 months after creating osteochondral defects.

A. Untreated osteochondral defect (Stain, thionine; original magnification, X25)

B. 55/45 treated osteochondral defect (Stain, thionine; original magnification, X25)

C. $70 / 30$ treated osteochondral defect (Stain, thionine; original magnification, X25). The defect contains intensively stained cartilage-like tissue (CT), which extended into the subchondral bone. Bone tissue (BT) was situated between cartilage-like tissue and scaffold remnants (S).

D. An enlargement of the box in (A) (stain, thionine; original magnification, X100). The defect is partly filled with reparative tissue consisting of fibrous tissue (FT) in the superficial zone and cartilage-like tissue (CT) containing cysts in the deeper layers.

E. An enlargement of the box in (B) (Stain, thionine; original magnification, X100). The defect contains well-organized bone tissue (BT) with fibrous tissue (FT) on top. Scaffold remnants (S) were observed throughout the osteochondral defect.

F. An enlargement of the box in (C) (Stain, thionine; original magnification, X100). 


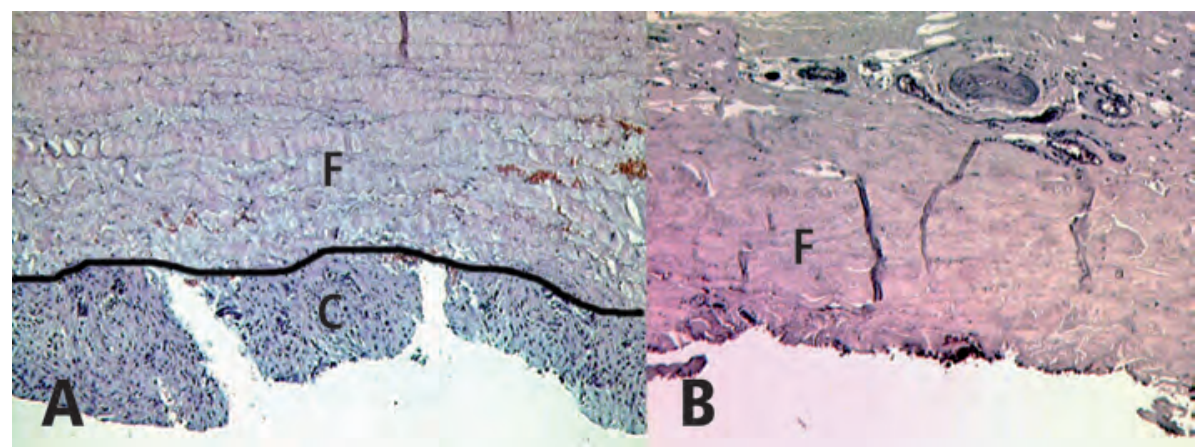

Figure 1. Micrographs of periosteum explants harvested from A) a young patient (age 5 years) undergoing an epiphysiodesis using a staple technique, approximately $2 \mathrm{~cm}$ caudally from the proximal tibial growth plate; and B) the proximal tibia from an elderly patient (age 62 years). Notice the clearly distinguishable cambium layer (c) in the periosteum derived from the young patient and the hardly detectable cambium layer in the periosteum from the elderly patient. The overlying fibrous layer (F) contains fibroblasts. The line in A) separates the fibrous (F) and cambium (C) layer. Magnification: 50X
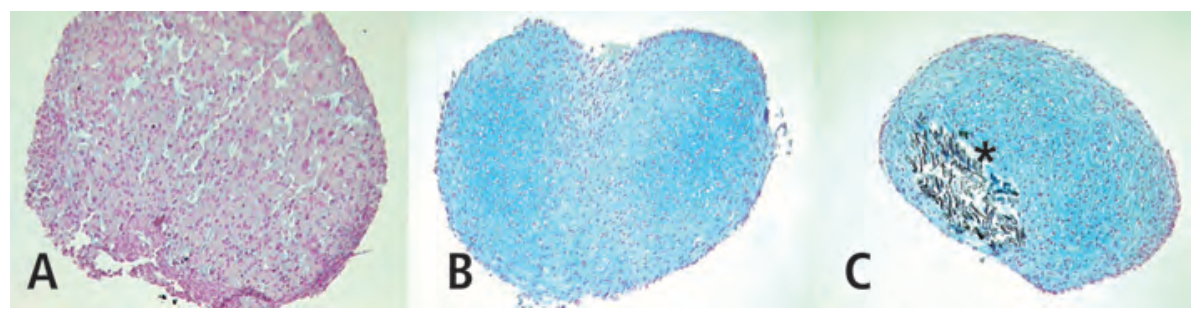

Figure 3. Micrographs of cryosections of micromasses after staining with Alcian blue (donor age was 62 years). Periosteum-derived cells were differentiated in medium A) without growth factors or medium supplemented with B) TGF $\beta_{1}(10 \mathrm{ng} / \mathrm{mL})$ or C) TGF $\beta_{3}(10 \mathrm{ng} / \mathrm{mL})$. Notice the zone of calcification $\left(^{*}\right)$ in the micromass that was differentiated with $\mathrm{TGF}_{3}$.

Magnification: X50 


\section{Chapter 6}
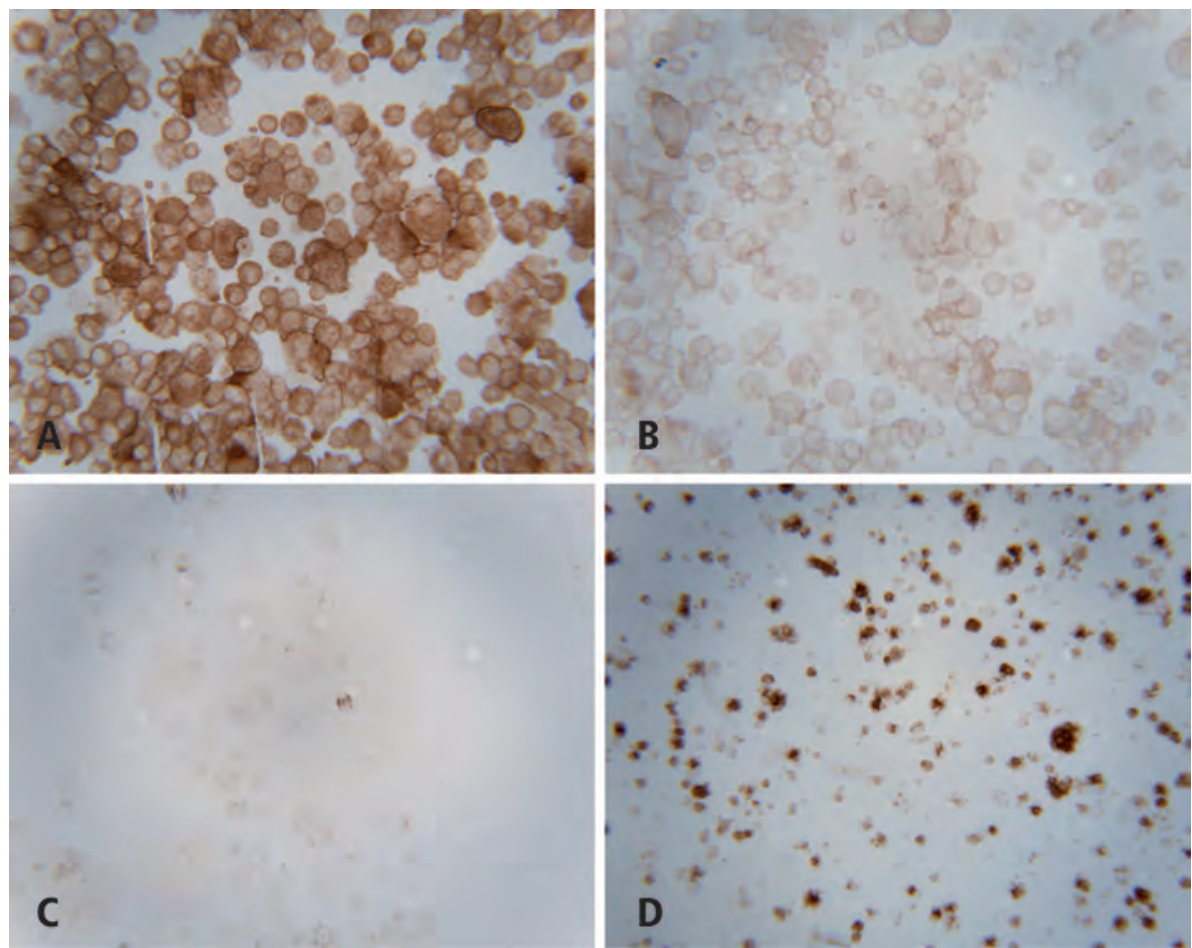

Figure 4. Cytospins of (A) human embryonic lung fibroblasts stained with an antibody for Thy-1 (positive control); (B) periosteum-derived cells from elderly patient stained with anti-Thy-1; (C) periosteum-derived cells from elderly patient stained with anti-collagen type I (M38, DSHB); (D) periosteum-derived cells from elderly patient stained with anti-collagen type II (II-II6B3, DSHB). Secondary antibodies were conjugated with horseradish peroxidase. Diaminobenzidine was used to develop the colour (brown is positive signal). 


\section{Chapter 7}
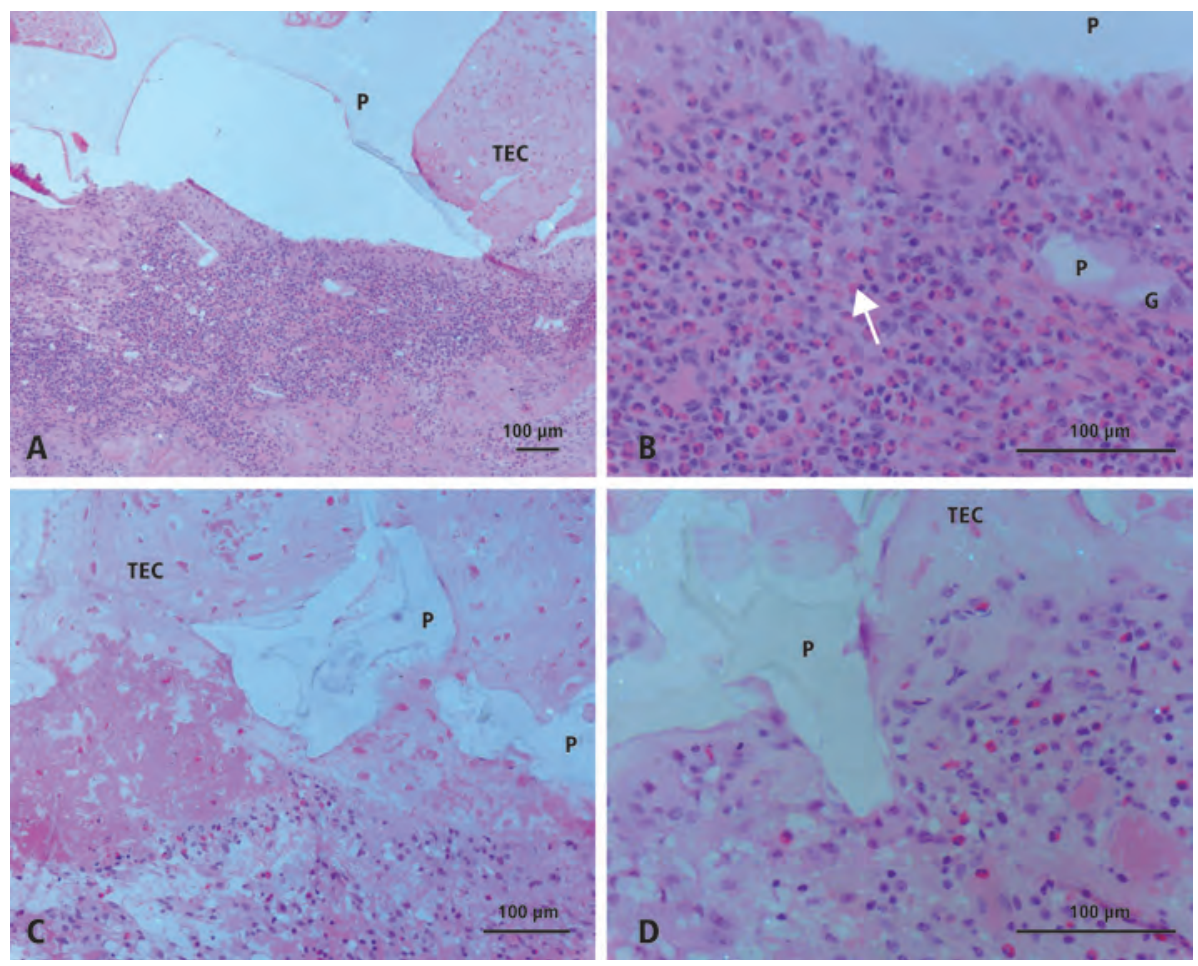

Figure 2. Histological observations of infected areas near an implanted PEOT/PBT 1000 60/40 scaffold with seeded and cultured allogeneic chondrocytes at 3 months follow-up. A - infected tissue with many polymorphonuclear cells and macrophages; $B$ - detail of $A$; $C$ - mild inflammatory reaction due to necrotic tissue-engineered cartilage; $\mathrm{D}$ - detail of $\mathrm{C}$ : limited number of polymorphonuclear cells present. P: Biomaterial, PEOT/PBT scaffold; G: giant cell; TEC: tissueengineered cartilage; Arrow: polymorphonuclear cell. 


\section{Chapter 8}
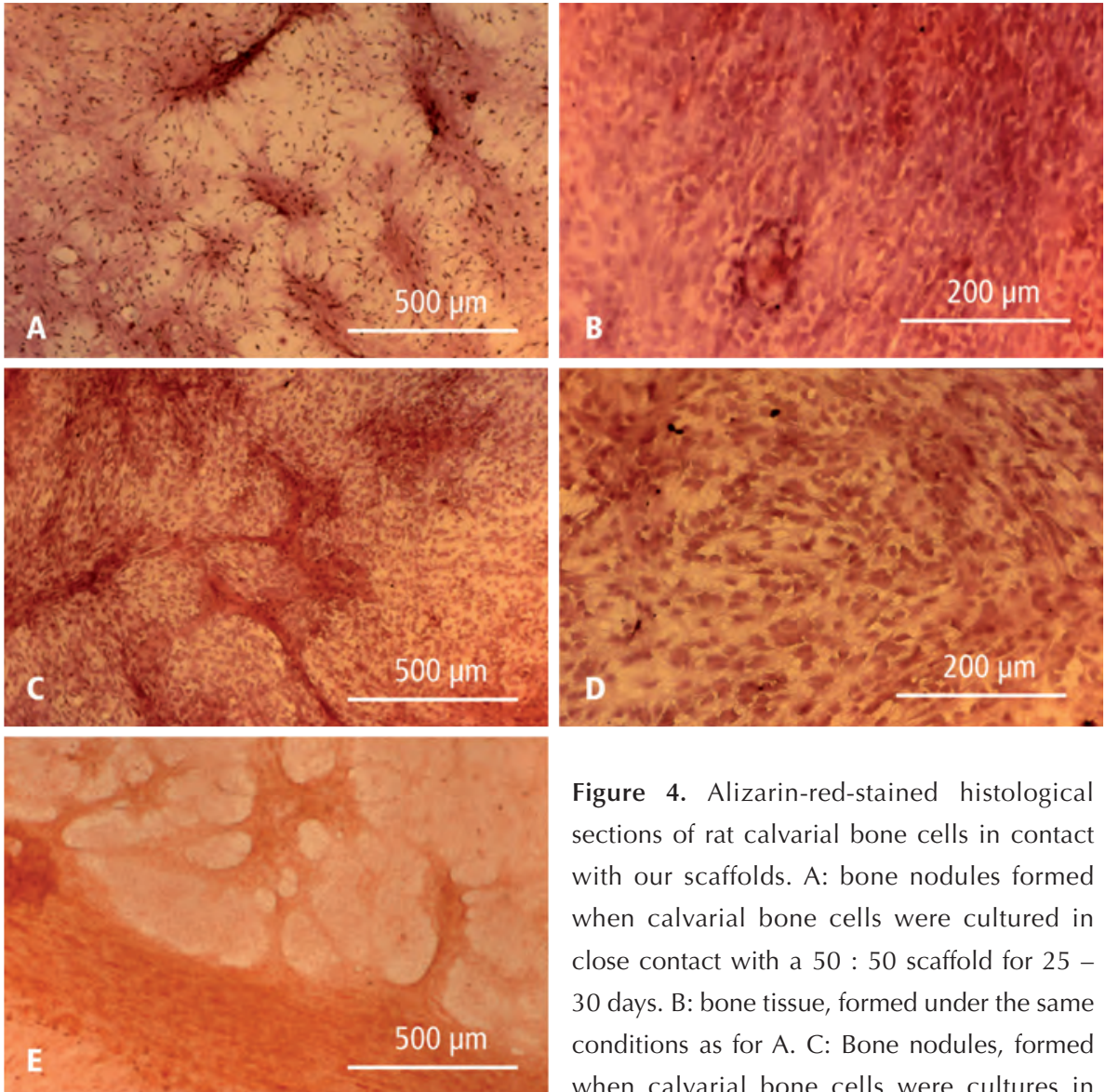

Figure 4. Alizarin-red-stained histological sections of rat calvarial bone cells in contact with our scaffolds. A: bone nodules formed when calvarial bone cells were cultured in close contact with a $50: 50$ scaffold for 25 30 days. B: bone tissue, formed under the same conditions as for A. C: Bone nodules, formed when calvarial bone cells were cultures in close contact with a $70: 30$ scaffold for 25 - 30 days. D: Cubical cells, formed under the same conditions as for C. E: Cell attachment onto the $50: 50$ scaffold.

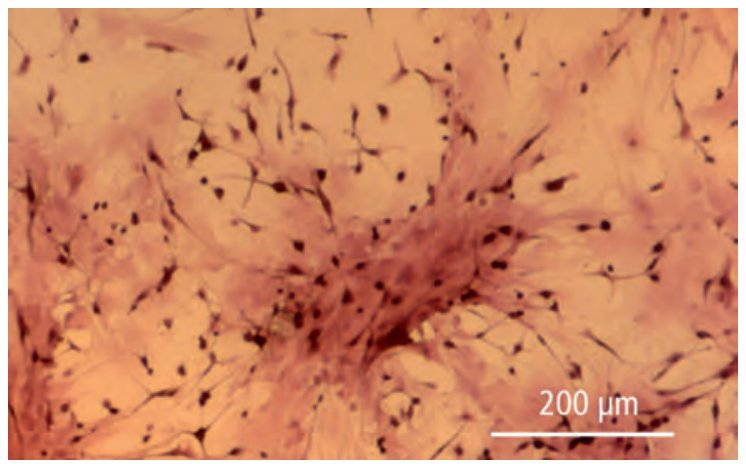

Figure 5. Alizarin-red-stained histological section of rat bone marrow cells cultured in contact with a 70 : 30 scaffold for 23 days. Fibroblast-like cells are present. No bone nodules are seen. 


\section{Chapter 8}
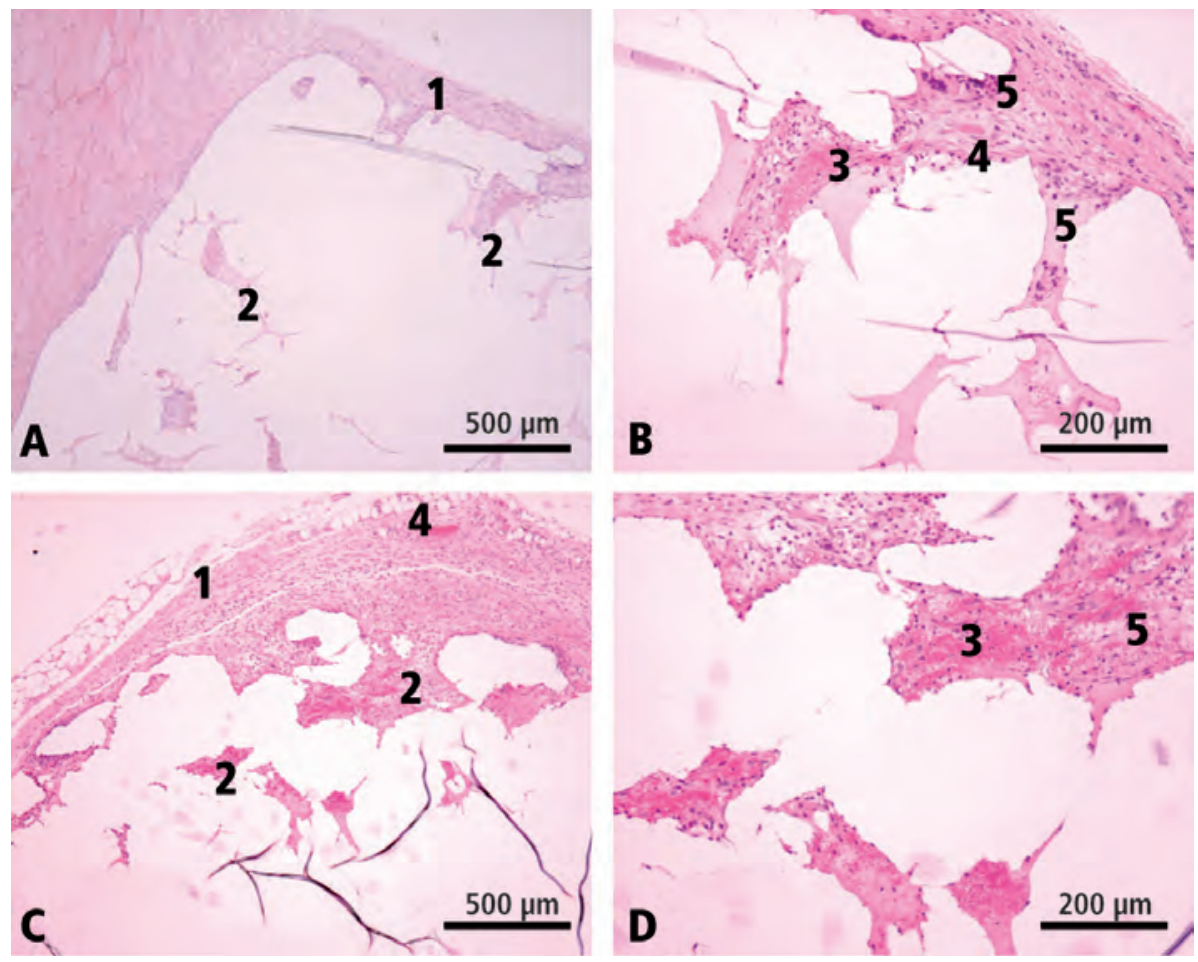

Figure 6. Haematoxylin/eosin-stained sections of specimens which were harvested 1 week after subcutaneous implantation in rats. A, B: $50: 50$ scaffold shown at different magnifications. C, D: 70 : 30 scaffold shown at different magnifications. All scaffolds are surrounded by fibrous tissue (1). Note fibroblast infiltration (2) and erythrocytes (3) in the peripheral pores of both scaffolds. Also, formation of blood capillaries (4) and some multinuclear cells (5) are seen. 


\section{Chapter 8}
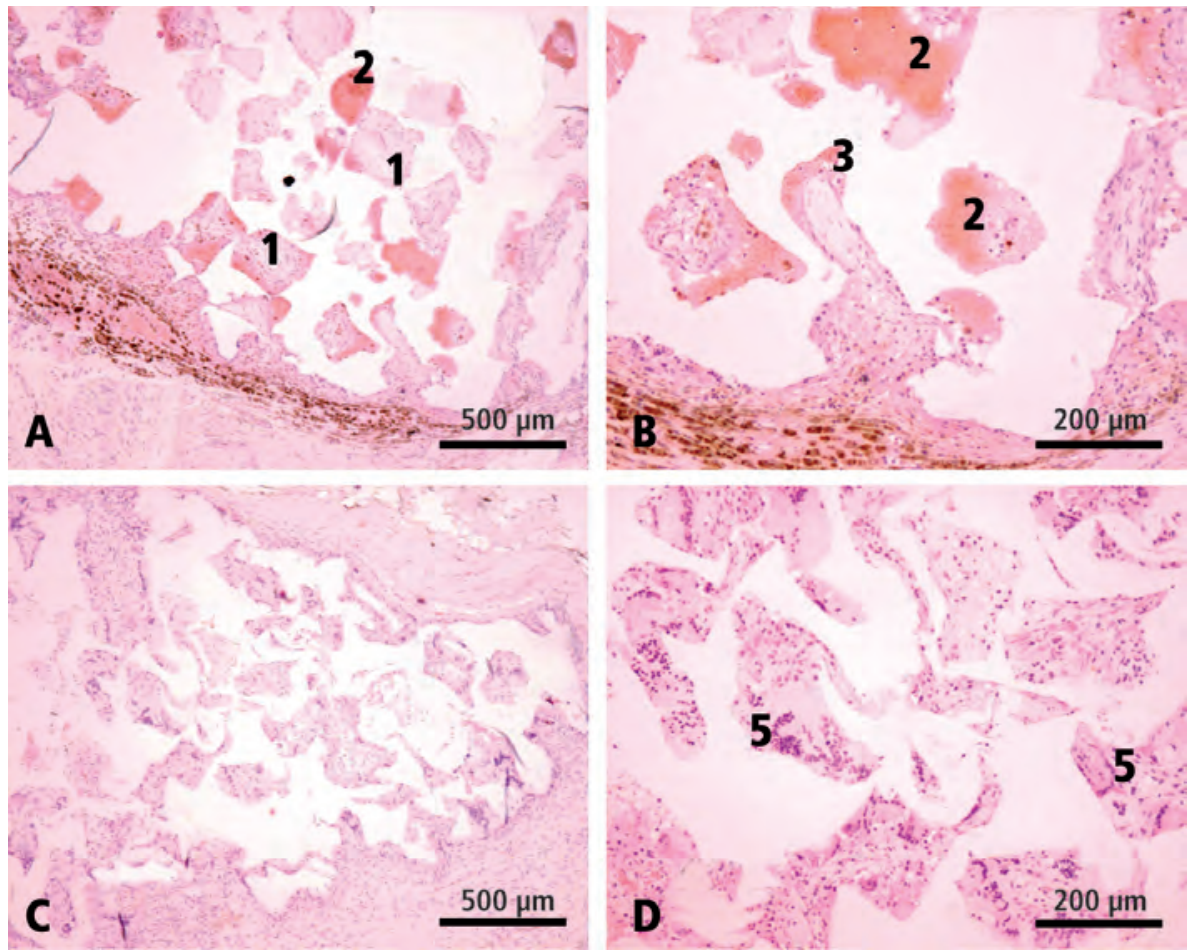

Figure 7. Haematoxylin/eosin-stained sections of specimens which were harvested 84 days after subcutaneous implantation in rats. A, B: $50: 50$ scaffolds shown at different magnifications. Note that the pores retained their rectangular shape (1). The pores are invaded by erythrocytes (2), endothelial cells and fibroblasts (3). Note the iron-containing macrophages (4) between the dermis and the $50: 50$ scaffold. C, D: $70: 30$ scaffolds shown at different magnifications. The pores in these scaffolds lost their rectangular shape, due to the softer nature of this material. The cavities are filled with fibrous tissue and many giant cells (5). 


\section{Chapter 8}
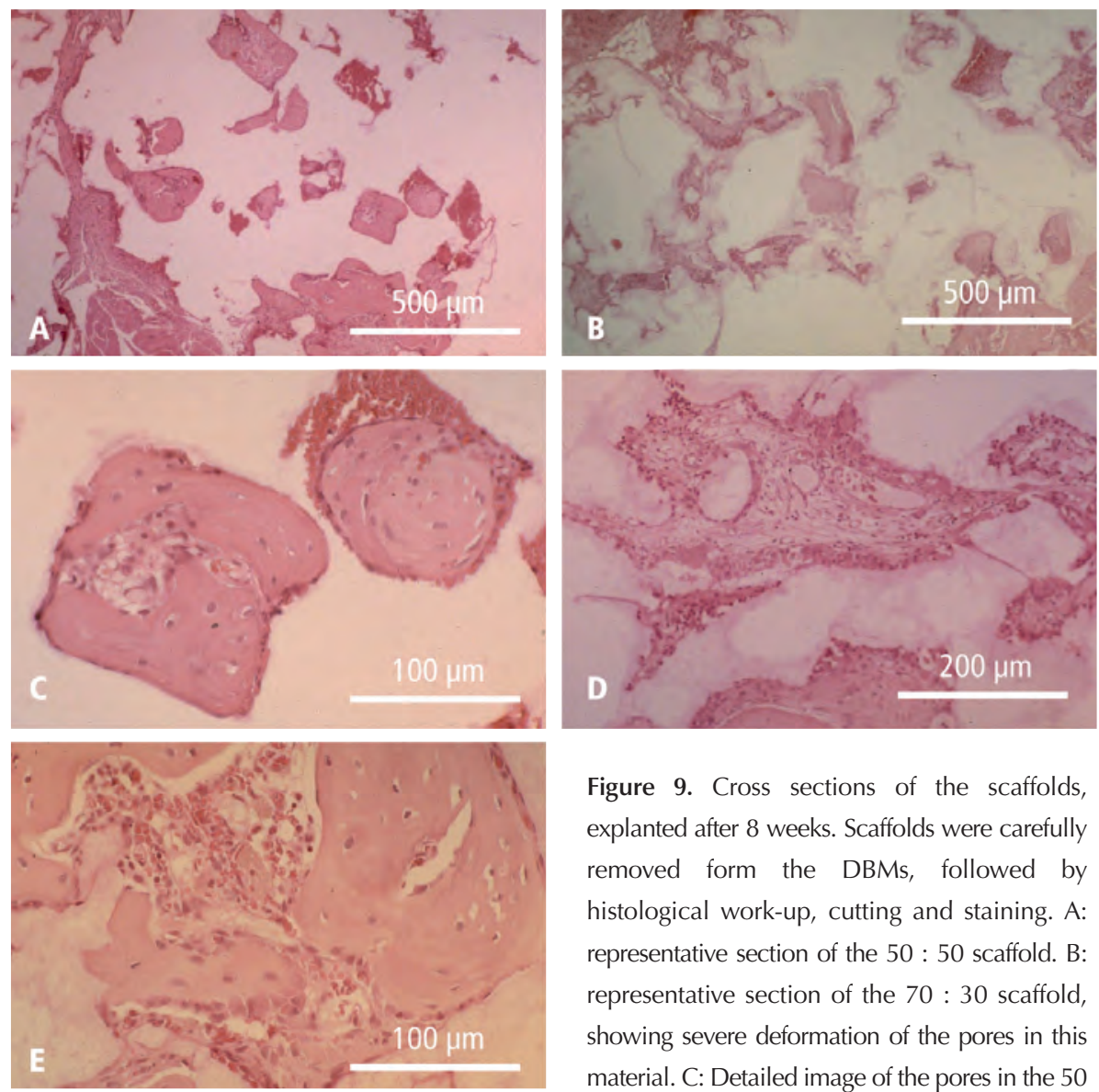

Figure 9. Cross sections of the scaffolds, explanted after 8 weeks. Scaffolds were carefully removed form the DBMs, followed by histological work-up, cutting and staining. A: representative section of the $50: 50$ scaffold. B: representative section of the $70: 30$ scaffold, showing severe deformation of the pores in this material. C: Detailed image of the pores in the 50

: 50 material. D: Detailed image of the pores in the $70: 30$ material. E: Newly formed bone in $50: 50$ scaffold. 
